\title{
Characterization of the Fuel Injection Process within the Ignition Quality Tester (IQT ${ }^{\mathrm{TM}}$ )
}

by

\section{Stephane Gilles Daviault}

\author{
A Thesis submitted to \\ the Faculty of Graduate Studies and Research \\ in partial fulfilment of \\ the requirements for the degree of \\ Master of Applied Science \\ Ottawa-Carleton Institute for \\ Mechanical and Aerospace Engineering
}

Department of Mechanical and Aerospace Engineering

Carleton University

Ottawa, Ontario, Canada

2011

Copyright (C)

2011 - Stephane Gilles Daviault 
Library and Archives Canada

Published Heritage Branch

395 Wellington Street Ottawa ON K1A ON4 Canada
Bibliothèque et

Archives Canada

Direction du

Patrimoine de l'édition

395 , rue Wellington

Ottawa ON K1A ON4

Canada
Your file Votre référence

ISBN: 978-0-494-83053-6

Our file Notre référence

ISBN: 978-0-494-83053-6
NOTICE:

The author has granted a nonexclusive license allowing Library and Archives Canada to reproduce, publish, archive, preserve, conserve, communicate to the public by telecommunication or on the Internet, loan, distribute and sell theses worldwide, for commercial or noncommercial purposes, in microform, paper, electronic and/or any other formats.

The author retains copyright ownership and moral rights in this thesis. Neither the thesis nor substantial extracts from it may be printed or otherwise reproduced without the author's permission.
AVIS:

L'auteur a accordé une licence non exclusive permettant à la Bibliothèque et Archives Canada de reproduire, publier, archiver, sauvegarder, conserver, transmettre au public par télécommunication ou par l'Internet, prêter, distribuer et vendre des thèses partout dans le monde, à des fins commerciales ou autres, sur support microforme, papier, électronique et/ou autres formats.

L'auteur conserve la propriété du droit d'auteur et des droits moraux qui protège cette thèse. $\mathrm{Ni}$ la thèse ni des extraits substantiels de celle-ci ne doivent être imprimés ou autrement reproduits sans son autorisation.
In compliance with the Canadian Privacy Act some supporting forms may have been removed from this thesis.

While these forms may be included in the document page count, their removal does not represent any loss of content from the thesis.
Conformément à la loi canadienne sur la protection de la vie privée, quelques formulaires secondaires ont été enlevés de cette thèse.

Bien que ces formulaires aient inclus dans la pagination, il n'y aura aucun contenu manquant.

\section{Canadä}


The undersigned recommend to

the Faculty of Graduate Studies and Research

acceptance of the Thesis

\title{
Characterization of the Fuel Injection Process within the Ignition Quality Tester (IQT ${ }^{\mathrm{TM}}$ )
}

\author{
Submitted by Stephane Gilles Daviault \\ in partial fulfilment of the requirements for the degree of \\ Master of Applied Science
}

E. Matida, Supervisor

M. Yaras, Department Chair

Carleton University

2011 


\section{Abstract}

The Ignition Quality Tester (IQT ${ }^{\mathrm{TM}}$ ) is a constant volume combustion chamber apparatus developed to measure the ignition delay of compression ignited fuels. The instrument provides a derived cetane number (DCN), which is correlated to the $\mathrm{CN}$ of the Cooperative Fuel Research (CFR) Engine. The importance of the IQT ${ }^{\mathrm{TM}}$ in the research sector is growing rapidly. Many research institutions in the fuel and combustion sector now use the $\mathrm{IQT}^{\mathrm{TM}}$ for parametric studies characterizing the ignition delay of newly developed fuels. The IQT ${ }^{\mathrm{TM}}$ has the potential of experimentally validating the combustion kinetic models of these new fuels developed from biomass. The temperatures, pressure, oxygen content and mass injected are all known and controllable variables in the IQT ${ }^{\mathrm{TM}}$ which makes model development simple. In addition, the lack of turbulence of the stagnate ambient air of the combustion apparatus also simplifies modeling. Researchers have modelled the ignition delay process within the $\mathrm{IQT}^{\mathrm{TM}}$. However, discrepancies were found between the experimental and modeled ignition delay due to the lack of spray characteristics creating large uncertainty in the physical breakup, evaporation and mixing process. Validating experimental results from the $\mathrm{IQT}^{\mathrm{TM}}$ to combustion kinetic models without an accurate physical characterization of the injection spray is difficult.

In this study, the injection spray of the IQT ${ }^{\mathrm{TM}}$ is characterized under atmospheric back pressure using Phase Doppler Anemometry (PDA, Dantec Dynamics). Viscor 
calibration fluid, which has diesel like fluid properties, is injected using the complete injection system of the IQT ${ }^{\mathrm{TM}}$. The high temporal resolution of the PDA method allowed precise time resolved analysis of droplet size and velocity. The arithmetic mean diameter (AMD) and Sauter mean diameter (SMD) of fuel droplets are found to be $9.3 \mu \mathrm{m}$ and $29.3 \mu \mathrm{m}$, respectively. The velocity is analyzed as a function of radial position from the nozzle axis. The droplet velocity varied between $130 \mathrm{~m} / \mathrm{s}$ and $10 \mathrm{~m} / \mathrm{s}$ depending on spatial position and time after the start of injection. Average velocities on the axis were found to be approximately $86 \mathrm{~m} / \mathrm{s}$, decreasing to $15 \mathrm{~m} / \mathrm{s}$ moving towards the outer spray periphery. The droplet count as a function of radial position suggests that most droplets form a cone angle of $12.6^{\circ}$, which is in agreement with a series of high speed images captured using front light photography. A decrease in the amount of mass injected is found to reduce the AMD while increasing the SMD. This has an adverse effect on the dispersity in the droplet size distribution. On the other hand, an increase of the injection pump accumulator pressure is found to reduce both the AMD and SMD of droplets while maintaining adequate droplet size distributions. A set of correlation equations of droplet size, velocity and distribution is then developed for researchers modelling the ignition process within the IQT ${ }^{\mathrm{TM}}$. 


\section{Acknowledgments}

I would like to thank professor Edgar A. Matida for making this project possible. Your support was greatly appreciated throughout my graduate studies. I would also thank Mr. Gary Webster of Advanced Engine Technology ltd. for his generous financial contribution towards the MITACS accelerate program. Also, in providing us with a complete IQT $\mathrm{T}^{\mathrm{TM}}$ injection apparatus in our laboratory for the duration of the work performed for this thesis.

J'aimerais remercier mes parents pour leur généreux support financier lors de mon Baccalaureat. Je voudrais également souligner l'appui de mes deux soeurs, dont je suis très fières. Caroline, merci pour ta présence durant les dernieres annees. Tu crois toujours en moi sans aucun doute. 


\section{Table of Contents}

$\begin{array}{ll}\text { Abstract } & \text { iii }\end{array}$

Acknowledgments $\quad$ v

Table of Contents vi vi

List of Tables $\quad$ x

List of Figures $\quad$ xi

Nomenclature $\quad$ xvi

1 Introduction $\quad 1$

1.1 ASTM Ignition Quality Determination . . . . . . . . . . . . . 1

1.1.1 Cooperative Fuel Research (CFR) Engine ASTM-D613 . . . . 1

1.1.2 Ignition Quality Tester (IQT $\left.{ }^{\mathrm{TM}}\right)$ ASTM-D6890 . . . . . . 2

1.2 Previous Work Completed . . . . . . . . . . . . . . . . . . 4

1.3 Thesis Objective ......................... 5

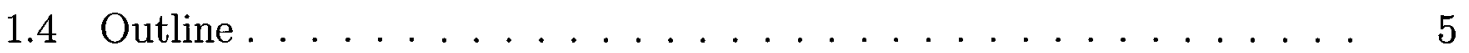

2 Background and Literature Review $\quad 8$

2.1 Ignition Quality Tester IQT $\mathrm{IQT}^{\mathrm{TM}} \ldots \ldots \ldots$

2.2 Diesel Spray Breakup . . . . . . . . . . . . . . . . . 12 
$2.2 .1 \quad$ Primary Breakup . . . . . . . . . . . . . . . . . 13

2.2 .2 Secondary Breakup . . . . . . . . . . . . . . . . 13

2.2 .3 Evaporation / Mixing . . . . . . . . . . . . . 15

2.3 Diesel Spray Penetration . . . . . . . . . . . . . . . . . . 17

2.4 Measurement techniques for sprays . . . . . . . . . . . 18

2.4.1 Planar Mie Scattering . . . . . . . . . . . . . 18

2.4.2 Back lit imaging (Shadowgraphy) . . . . . . . . . . . 19

2.4.3 Particle Image Velocimetry (PIV) . . . . . . . . . . . . 20

2.4.4 Laser Diffraction and Laser Interferometry . . . . . . . . . 20

3 Laser and Phase Doppler Anemometry 22

3.1 Measurement Principle . . . . . . . . . . . . . . . . . . . . . 22

3.1.1 Laser Doppler Anemometry . . . . . . . . . . . . . . 23

3.1 .2 Phase Doppler Anemometry . . . . . . . . . . . . . 26

4 Experimental Apparatus $\quad 32$

4.1 Injection Apparatus . . . . . . . . . . . . . . . . . . 33

4.1 .1 Injection Pump . . . . . . . . . . . . . 35

4.1 .2 Injection Nozzle . . . . . . . . . . . . . . . . 36

4.2 Electronics . . . . . . . . . . . . . . . . . . . . . . . 37

4.2.1 Digital Signal and Data Acquisition . . . . . . . . . . . 37

$4.2 .2 \quad$ Needle Lift Sensor . . . . . . . . . . . . . . . . . 37

4.2 .3 Custom Schmitt Trigger . . . . . . . . . . . . . . 38

4.3 Characterizing Instruments . . . . . . . . . . . . . . . . . . 39

4.3.1 CCD High Speed Camera . . . . . . . . . . . . . . . 39

$4.3 .2 \quad$ Phase Doppler Anemometry . . . . . . . . . . . . . . . . 40

4.4 Summary of Process Diagram . . . . . . . . . . . . . . 42 
5.1 Droplet Scattering Properties . . . . . . . . . . . . . . . . 44

5.1 .1 Scattering Modes . . . . . . . . . . . . . . . . 47

$5.1 .2 \quad$ Scattering Angle Selection . . . . . . . . . . . . . . . 49

5.2 Selection of Instrument Parameters _ . . . . . . . . . . . 50

5.2 .1 Laser Power . . . . . . . . . . . . . . . . . . . 50

5.2 .2 High Voltage, and SNR . . . . . . . . . . . . 51

$5.2 .3 \quad$ Receiver Slit Width . . . . . . . . . . . . . . . . . . . 52

5.2 .4 Injection Sample Size . . . . . . . . . . . . . . . . 54

5.2 .5 Transmitting Optics $\ldots \ldots \ldots \ldots \ldots$

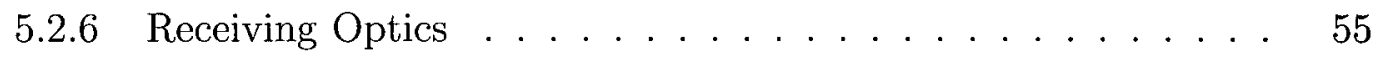

5.2 .7 Processor Limitations . . . . . . . . . . . . . . . . . 57

5.2 .8 Signal Validation Process . . . . . . . . . . . . . . . . 59

5.3 Parameter Selection Summary . . . . . . . . . . . . . . . . . . 61

5.4 Uncertainty Analysis . . . . . . . . . . . . . . . . . . 62

5.4 .1 Velocity error . . . . . . . . . . . . . 62

5.4 .2 Diameter ....................... 63

6 Results $\quad 66$

6.1 Data Analysis . . . . . . . . . . . . . . . . . . . 66

6.1 .1 Injector Dynamics $\ldots \ldots \ldots \ldots 6$

$6.1 .2 \quad$ Analysis Procedure . . . . . . . . . . . . 68

6.1 .3 Calculated Variables . . . . . . . . . . . . . . . 72

6.2 Spray Characteristics at Standard IQT ${ }^{\mathrm{TM}}$ Setting . . . . . . . 76

6.2.1 Qualitative Characteristics from High Speed Imaging . . . . 76

6.2 .2 Radial Analysis of PDA Measurements . . . . . . . . . 78

6.2 .3 Time Resolved Analysis _ . . . . . . . . . . . . . . 81 
6.3 Effect of Mass Injected on Atomization . . . . . . . . . . . . 85

6.4 Effect of the Accumulator Pressure on Atomization . . . . . . . . 88

6.5 Effect of ambient pressure on spray tip penetration . . . . . . . 91

6.6 Effect of ambient pressure on droplet size . . . . . . . . . . . . . 94

6.7 Backtracking Algorithm _. . . . . . . . . . . . . . . 95

6.8 Discharged velocity approximation $\ldots \ldots \ldots \ldots$

$6.9 \mathrm{IQT}^{\mathrm{TM}}$ injection rate $\ldots \ldots \ldots \ldots \ldots \ldots$

6.10 Correlated Characteristic Equations . . . . . . . . . . . . . . . 102

7 Conclusions $\quad 104$

7.1 Summary . . . . . . . . . . . . . . . . . . . . 104

7.1.1 Qualitative Characteristics . . . . . . . . . . 104

7.1.2 Quantitative Characteristics ... . . . . . . . . . . 104

7.2 Recommendations . . . . . . . . . . . . . . . . 106

7.3 Future Work . . . . . . . . . . . . . . . . . . . . 107

$\begin{array}{ll}\text { List of References } & 108\end{array}$

$\begin{array}{lr}\text { Appendix A Fluid Properties } & 115\end{array}$

Appendix B Phase Doppler Anemometry Experimental Data 116

B.1 PDA results at standard mass, standard injection energy . . . . . 116

B.1.1 $x=35 \mathrm{~mm} \ldots \ldots \ldots \ldots \ldots \ldots \ldots$

B.1.2 $x=55 \mathrm{~mm} \ldots \ldots \ldots \ldots \ldots \ldots \ldots \ldots$

B.1.3 $x=70 m m \ldots \ldots \ldots \ldots \ldots \ldots \ldots$

B.1.4 $x=85 \mathrm{~mm} \ldots \ldots \ldots \ldots \ldots \ldots \ldots$

$\begin{array}{ll}\text { Appendix C Time resolved velocity profile } & 157\end{array}$

$\begin{array}{ll}\text { Appendix D PDA Matlab Algorithm } & 161\end{array}$ 


\section{List of Tables}

5.1 Measurement volume dimension comparison . . . . . . . . . . . 55

5.2 Maximum droplet size vs mask selection . . . . . . . . . . . . 56

5.3 Summary of instrument parameter selection $\ldots \ldots \ldots 61$

5.4 Component error summary . . . . . . . . . . . . . . . . . . 63

6.1 Measurement position . . . . . . . . . . . . . . . 71

6.2 Result summary, standard injection parameters. Droplet size uncertainty found using analysis in Section 5.4.2. . . . . . . 80

6.3 Result summary, effect of mass injected . . . . . . . . . . . . 88

6.4 Result summary, effect of injection pressure . . . . . . . . . . . 90

6.5 Effect of ambient pressure on spray tip penetration $\ldots \ldots \ldots . . .92$

6.6 Average axial velocity . . . . . . . . . . . . . . . 96

D.1 Example of raw data from the BSA flow software $\ldots \ldots \ldots 161$ 


\section{List of Figures}

$2.1 \mathrm{IQT}^{\mathrm{TM}}$ instrument $\ldots \ldots \ldots \ldots \ldots \ldots$

$2.2 \mathrm{IQT}^{\mathrm{TM}}$ schematic $[1] \ldots \ldots \ldots \ldots \ldots$

2.3 IQT $^{\mathrm{TM}}$ Ignition delay determination ASTM D6890 [2] [1]. Solid line represents the needle lift. Dashed line represents the chamber pressure. 11

2.4 Diesel spray combustion . . . . . . . . . . . . . . . . . . 12

2.5 Regime of cavitating and non-cavitating flow through Diesel nozzles [3] 14

2.6 Droplet secondary breakup regimes. Adapted from: [4] . . . . . . 15

2.7 Techniques for converting a laser beam into a sheet $\ldots \ldots \ldots$

3.1 Measurement volume from intersecting coherent laser beams . . . . . 23

3.2 Single beam LDA principle . . . . . . . . . . . . . . . . . 24

3.3 Modern two beam LDA configuration . . . . . . . . . . . . . 25

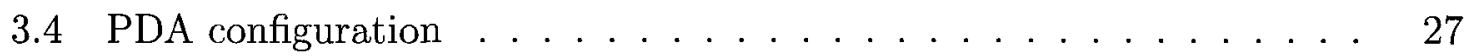

3.5 Detector signal phase difference. Adapted from [5] . . . . . . . . 28

3.6 Receiving Optics mask . . . . . . . . . . . . . . . . . . . . 29

$3.72 \pi$ ambiguity. Adapted from $[5] \ldots \ldots \ldots \ldots \ldots$

3.8 Solution to the " $2 \pi$ ambiguity" problem using 3 detectors . . . . . . . 31

4.1 Measurement point grid . . . . . . . . . . . . . . . . . 32

$4.2 \mathrm{IQT}^{\mathrm{TM}}$ injection apparatus . . . . . . . . . . . . 34

$4.3 \mathrm{IQT}^{\mathrm{TM}}$ injection pump $\ldots \ldots \ldots \ldots \ldots \ldots \ldots \ldots$

$4.4 \mathrm{IQT}^{\mathrm{TM}}$ injection nozzle $\ldots \ldots \ldots \ldots \ldots \ldots \ldots$ 
4.5 Custom Schmitt trigger and monostable vibrator circuit . . . . . . . . 38

4.6 Nozzle needle trace and trigger threshold voltage . . . . . . . . . . . . 40

4.7 PDA system and experimental apparatus . . . . . . . . . . . . . 42

4.8 Experimental process diagram . . . . . . . . . . . . . . . 43

5.1 Scattered light intensity as a function of Mie parameter $x_{m}$ and particle size for water $n_{r e l}=1.33$. Solid line represents $\theta=30^{\circ}$ and parallel polarization $[6] \ldots \ldots \ldots \ldots$

5.2 Scattering intensity for three different Mie parameter values computed for water droplets in air using EGO [6] . . . . . . . . . . . . . . . . . 46

5.3 Scattered light from water droplet where $d_{p} \gg \lambda_{b}, n_{r e l}=1.33[5]$. . . 47

5.4 Intensity distribution of different scattering modes for $d_{p}=10 \mu \mathrm{m}$ water droplet calculated using extended geometrical optics EGO. [6] .

5.5 Droplet of diesel oil in atmospheric air $n_{\text {rel }}=1.46$. Left: Logarithmic light intensity. Right: GO representation [5] . . . . . . . . .

5.6 Effect of laser power on the droplet AMD and velocity . . . . . . . . 51

5.7 FiberFlow PDA Probe (57X80) layout. Photo detectors are represented as U1,U2 and U3. . . . . . . . . . . . . . .

5.8 Importance of the slit aperture for Gaussian intensity distributed measurement volumes. Adapted from Wigley et al. [7] . . . . . . . . .

5.9 Influence of the receiving optics slit aperture: velocity results $40 \mathrm{~mm}$ downstream from the orifice, $1.4 \mathrm{~mm}$ off-axis, $P_{\imath n_{\jmath}}=500 \mathrm{bar}, P_{a m b}=$ 7 bar (air) [8] ......................... 54

5.10 AMD and average axial velocity as a function of injection sample size. $\quad 54$

5.11 Diameter histogram and time resolved droplet size (30 injections Mask

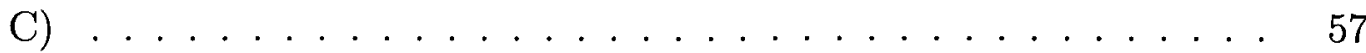

5.12 Data coupling analysis (concatenation process) . . . . . . . . 58

5.13 Doppler burst and noise contribution [6] . . . . . . . . . . . . 59 
5.14 Signal to noise ratio $[6] \ldots \ldots \ldots \ldots$

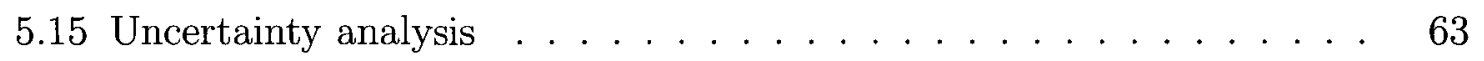

6.1 Nozzle needle dynamic behaviour . . . . . . . . . . . . . 67

6.2 Spray image synchronized to the needle lift trace . . . . . . . . . 67

6.3 Velocity data at $85 \mathrm{~mm}$ from nozzle tip and $0 \mathrm{~mm}$ radial . . . . . . 69

6.4 Coupling injector needle position to the PDA data at $x=85 \mathrm{~mm}$,

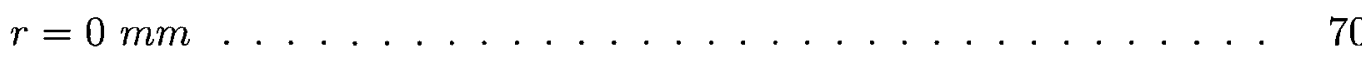

6.5 Bin averaged axial velocity, AMD and SMD for the optimization of the time bin width selection . . . . . . . . . . . . 75

6.6 Side view of the injection spray in atmospheric ambient air at standard $\mathrm{IQT}^{\mathrm{TM}}$ setting . . . . . . . . . . . . . . 77

6.7 Axial velocity vs radial position $\ldots \ldots \ldots \ldots \ldots \ldots$

6.8 AMD and dimensionless droplet count as a function of nondimensional radial position $r^{\prime} \ldots \ldots \ldots \ldots \ldots \ldots$

6.9 Velocity profiles $0.2 \mathrm{~ms}$ to $1.0 \mathrm{~ms} \ldots \ldots \ldots$

6.10 Velocity profiles $1.2 \mathrm{~ms}$ to $2.4 \mathrm{~ms} \ldots \ldots \ldots \ldots$

6.11 Velocity RMS at $x=35 \mathrm{~mm} \ldots \ldots \ldots \ldots \ldots$

6.12 Effect of mass injected on the needle trace and droplet count . . . . 86

6.13 Effect of mass injected on the axial velocity and AMD $\ldots \ldots$. . . 87

6.14 Effect of injection pressure on the needle lift and droplet count . . . . 89

6.15 Effect of injection pressure on the axial velocity and AMD . . . . . 91

6.16 Optical chamber . . . . . . . . . . . . . . . . . . 91

6.17 Effect of the ambient pressure on the penetration rate . . . . . . 92

6.18 Effect of ambient pressure on spray tip velocity $\ldots \ldots \ldots 93$

6.19 Effect of ambient pressure on SMD . . . . . . . . . . . . . 94

6.20 Effect of the Spalding number on spray penetration . . . . . . . . 99

$6.21 \mathrm{IQT}^{\mathrm{TM}}$ injection rate . . . . . . . . . . . . . . . 102 
6.22 Correlated spray characteristic equations . . . . . . . . . . 103

B.1 $\mathrm{x}=35 \mathrm{~mm}, \mathrm{r}=0 \mathrm{~mm} \ldots \ldots \ldots \ldots \ldots \ldots \ldots$

B.2 $\mathrm{x}=35 \mathrm{~mm}, \mathrm{r}=2 \mathrm{~mm} \ldots \ldots \ldots \ldots \ldots \ldots \ldots \ldots \ldots \ldots \ldots \ldots \ldots$

B.3 $\mathrm{x}=35 \mathrm{~mm}, \mathrm{r}=3 \mathrm{~mm} \ldots \ldots \ldots \ldots \ldots \ldots \ldots$

B.4 $\mathrm{x}=35 \mathrm{~mm}, \mathrm{r}=3.5 \mathrm{~mm} \ldots \ldots \ldots \ldots \ldots \ldots$

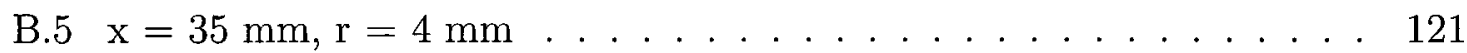

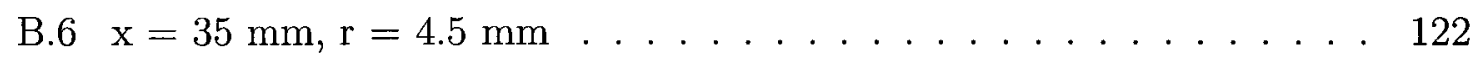

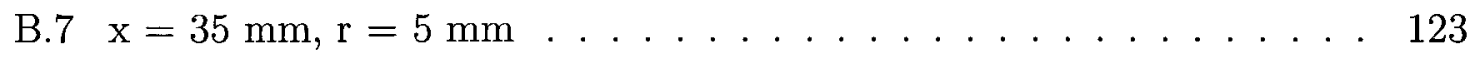

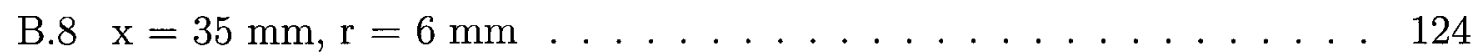

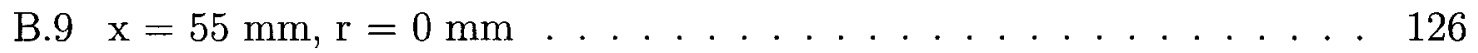

B.10 $\mathrm{x}=55 \mathrm{~mm}, \mathrm{r}=2.5 \mathrm{~mm} \ldots \ldots \ldots \ldots \ldots \ldots \ldots$

B. $11 \mathrm{x}=55 \mathrm{~mm}, \mathrm{r}=3.5 \mathrm{~mm} \ldots \ldots \ldots \ldots \ldots \ldots$

B.12 $\mathrm{x}=55 \mathrm{~mm}, \mathrm{r}=4.5 \mathrm{~mm} \ldots \ldots \ldots \ldots \ldots \ldots$

B.13 $\mathrm{x}=55 \mathrm{~mm}, \mathrm{r}=5.5 \mathrm{~mm} \ldots \ldots \ldots \ldots \ldots$

B.14 $\mathrm{x}=55 \mathrm{~mm}, \mathrm{r}=6.5 \mathrm{~mm} \ldots \ldots \ldots \ldots \ldots \ldots \ldots \ldots$

B. $15 \mathrm{x}=55 \mathrm{~mm}, \mathrm{r}=7.5 \mathrm{~mm} \ldots \ldots \ldots \ldots \ldots \ldots$

B.16 $\mathrm{x}=55 \mathrm{~mm}, \mathrm{r}=8.5 \mathrm{~mm} \ldots \ldots \ldots \ldots \ldots \ldots \ldots$

B.17 $\mathrm{x}=70 \mathrm{~mm}, \mathrm{r}=0 \mathrm{~mm} \ldots \ldots \ldots \ldots \ldots \ldots$

B. $18 \mathrm{x}=70 \mathrm{~mm}, \mathrm{r}=4.5 \mathrm{~mm} \ldots \ldots \ldots \ldots \ldots$

B.19 $\mathrm{x}=70 \mathrm{~mm}, \mathrm{r}=5.5 \mathrm{~mm} \ldots \ldots \ldots \ldots \ldots \ldots \ldots$

B. $20 \mathrm{x}=70 \mathrm{~mm}, \mathrm{r}=6.5 \mathrm{~mm} \ldots \ldots \ldots \ldots \ldots \ldots$

B. $21 \mathrm{x}=70 \mathrm{~mm}, \mathrm{r}=7.5 \mathrm{~mm} \ldots \ldots \ldots \ldots \ldots \ldots$

B. $22 \mathrm{x}=70 \mathrm{~mm}, \mathrm{r}=8.5 \mathrm{~mm} \ldots \ldots \ldots \ldots \ldots \ldots$

B. $23 \mathrm{x}=70 \mathrm{~mm}, \mathrm{r}=9.5 \mathrm{~mm} \ldots \ldots \ldots \ldots \ldots \ldots$

B. $24 \mathrm{x}=70 \mathrm{~mm}, \mathrm{r}=10.5 \mathrm{~mm} \ldots \ldots \ldots \ldots \ldots \ldots$

B. $25 \mathrm{x}=70 \mathrm{~mm}, \mathrm{r}=11.5 \mathrm{~mm} \ldots \ldots \ldots \ldots \ldots \ldots$

B. $26 \mathrm{x}=70 \mathrm{~mm}, \mathrm{r}=12.5 \mathrm{~mm} \ldots \ldots \ldots \ldots \ldots \ldots$ 
B. $27 \mathrm{x}=70 \mathrm{~mm}, \mathrm{r}=13.5 \mathrm{~mm} \ldots \ldots \ldots \ldots \ldots \ldots \ldots$

B. $28 \mathrm{x}=85 \mathrm{~mm}, \mathrm{r}=0 \mathrm{~mm} \ldots \ldots \ldots \ldots \ldots \ldots \ldots$

B. $29 \mathrm{x}=85 \mathrm{~mm}, \mathrm{r}=7.5 \mathrm{~mm} \ldots \ldots \ldots \ldots \ldots \ldots$

B. $30 \mathrm{x}=85 \mathrm{~mm}, \mathrm{r}=8.5 \mathrm{~mm} \ldots \ldots \ldots \ldots \ldots \ldots$

B.31 $\mathrm{x}=85 \mathrm{~mm}, \mathrm{r}=9.5 \mathrm{~mm} \ldots \ldots \ldots \ldots \ldots$

B. $32 \mathrm{x}=85 \mathrm{~mm}, \mathrm{r}=10.5 \mathrm{~mm} \ldots \ldots \ldots \ldots \ldots \ldots$

B.33 $\mathrm{x}=85 \mathrm{~mm}, \mathrm{r}=11.5 \mathrm{~mm} \ldots \ldots \ldots \ldots \ldots \ldots$

B. $34 \mathrm{x}=85 \mathrm{~mm}, \mathrm{r}=12.5 \mathrm{~mm} \ldots \ldots \ldots \ldots \ldots \ldots$

B. $35 \mathrm{x}=85 \mathrm{~mm}, \mathrm{r}=13.5 \mathrm{~mm} \ldots \ldots \ldots \ldots \ldots \ldots \ldots$

B.36 $\mathrm{x}=85 \mathrm{~mm}, \mathrm{r}=14.5 \mathrm{~mm} \ldots \ldots \ldots \ldots \ldots \ldots$

B. $37 \mathrm{x}=85 \mathrm{~mm}, \mathrm{r}=15.5 \mathrm{~mm} \ldots \ldots \ldots \ldots \ldots \ldots$

C.1 Time resolved velocity profile $0.1 \mathrm{~ms}$ to $0.8 \mathrm{~ms} \ldots \ldots \ldots \ldots$

C.2 Time resolved velocity profile $0.9 \mathrm{~ms}$ to $2.0 \mathrm{~ms} \ldots \ldots \ldots$. . . 158

C.3 Time resolved velocity profile $2.1 \mathrm{~ms}$ to $3.2 \mathrm{~ms} \ldots \ldots$. . . . . . 159

C.4 Time resolved velocity profile $3.3 \mathrm{~ms}$ to $4.4 \mathrm{~ms} \ldots \ldots \ldots$. . . . . 160 


\section{Nomenclature}

List of Abbreviations and Acronyms: The following acronyms are used throughout the thesis:

\begin{tabular}{llll}
\hline Acronym & Description & & \\
\hline AET & Advanced Engine Technology & SOI & Start Of Injection \\
IQT & Ignition Quality Tester & NOP & Nozzle Opening Pressure \\
ASTM & American Society of Testing and Materials & DAQ & Data Acquisition Card \\
CN & Cetane Number & FLMT & Fourier-Lorenz Mie Theory \\
DCN & Derived Cetane Number & GO & Geometrical Optics \\
PDA & Phase Doppler Anemometry & EGO & Extended Geometrical Optics \\
LDV & Laser Doppler Velocimetry & CW & Continuous Wave \\
CCD & Charged Coupled Device & HV & High Voltage \\
AMD & Arithmetic Mean Diameter & SNR & Signal to Noise Ratio \\
SMD & Sauter Mean Diameter & FFT & Fast Fourier Transform \\
CFR & Cooperative Fuel Research & PSD & Power Spectral Density \\
TDC & Top Dead Center & FPS & Frame Per Seconds \\
PIV & Particle Image Velocimetry & TTL & Transistor-Transistor-Logic
\end{tabular}


List of symbols: The following symbols are used throughout the thesis:

\begin{tabular}{llllll}
\hline Symbol & Description & Units & & & \\
\hline$W_{e}$ & Weber number & $\mathrm{NA}$ & $f_{o}$ & Bragg cell shifted frequency & $\mathrm{Hz}$ \\
$B$ & Spalding number & $\mathrm{NA}$ & $\phi$ & Beam intersection angle & $\mathrm{rad}$ \\
$Y_{R}$ & Droplet surface mass fraction & $\mathrm{NA}$ & $\theta$ & Scattering Angle & $\mathrm{rad}$ \\
$Y_{\infty}$ & Ambient air mass fraction & $\mathrm{NA}$ & $\psi$ & Inclination Angle & $\mathrm{rad}$ \\
$p_{v}$ & Vapor pressure & $P a$ & $\Phi$ & Phase difference & $\mathrm{rad}$ \\
$\rho_{f u e l}$ & Liquid fuel density & $k g / \mathrm{m}^{3}$ & $\beta$ & Detector geometrical factor & NA \\
$\rho_{a i r}$ & Ambient air density & $k g / \mathrm{m}^{3}$ & $n_{r e l}$ & Relative refraction index & NA \\
$G$ & Droplet surface mass flux & $k g / \mathrm{m}^{2} s$ & $X_{m}$ & Mie parameter & NA \\
$D_{a b}$ & Diffusivity of Diesel into Air & $\mathrm{m}^{2} / \mathrm{s}$ & $P_{1}$ & Fuel pressure & $b a r s$ \\
$K_{v}$ & Droplet evaporation rate & $\mathrm{m}^{2} / \mathrm{s}$ & $P_{2}$ & Ambient air pressure & $b a r s$ \\
$C_{d}$ & Drag Coefficient & $\mathrm{NA}$ & $A_{f}$ & Instantaneous flow area & $\mathrm{m}^{2}$ \\
$\lambda_{b}$ & Incident beam wavelength & $\mathrm{nm}$ & $C_{d \imath s}$ & Discharge coefficient & NA \\
$e$ & Incident beam velocity & $m / s$ & $u$ & Axial velocity & $\mathrm{m} / \mathrm{s}$ \\
$f_{r}$ & Frequency shift & $\mathrm{Hz}$ & $x$ & Axial distance from nozzle tip & $\mathrm{mm}$ \\
$f_{D}$ & Doppler frequency & $\mathrm{Hz}$ & $r$ & Radial distance from nozzle axis & $\mathrm{mm}$ \\
$\sigma_{g}$ & Dropelt size dispersity & $\mathrm{NA}$ & $\sigma_{d}$ & Standard dev. of droplet size & $\mu m$ \\
$\sigma_{D 10}$ & Standard dev. estimate of the AMD & $\mu m$ & & &
\end{tabular}




\section{Chapter 1}

\section{Introduction}

The introduction of the diesel engine in the 1920's revolutionized the commercial transportation industry. The application of the diesel engine is incredibly widespread from stationary power generation, rail locomotives, marine propulsion and the biggest mover of all, road transportation [9]. Diesel producers must ensure that the ignition quality of diesel fuel blends meet engine manufacturer's specification as pollution, particulate emission, power output and noise, which are all function of the ignition quality of the fuel [10]. Cetane Number $(\mathrm{CN})$ is a regulated specification that characterizes the ignition quality of diesel fuels. The $\mathrm{CN}$ is obtained by measuring the ignition delay from the start of injection (SOI) to the time of combustion. Fuels with a high $\mathrm{CN}$ will auto-ignite quicker and burn more completely to minimize the creation of undesired exhaust emissions.

\subsection{ASTM Ignition Quality Determination}

\subsubsection{Cooperative Fuel Research (CFR) Engine ASTM-D613}

The CN of diesel fuels were initially determined experimentally in a Cooperative Fuel Research (CFR) test engine according to the ASTM D613 method, which is 
a standard test method for Cetane Number determination of diesel fuel oil. The CFR engine consists of a single cylinder indirect injected compression ignition engine where the compression ratio is adjustable by varying the dead-space volume in the injection pre-chamber. The CFR engine is run at $900 \mathrm{rpm}$ with an injection timing set at $13^{\circ}$ before top dead center (TDC) which corresponds to an ignition delay of $2.407 \mathrm{~ms}$ [11]. The engine operator manually adjusts the compression ratio in order to obtain combustion of the sampled fuel at TDC. The sampled fuel's CN is then determined by formulating a blend of reference fuels that match the performance of the sample. One particular drawback of the CFR engine technology has been its reproducibility limits. The current method lists reproducibility limits that vary from $2.8 \mathrm{CN}$ at a $\mathrm{CN}$ level of $40 \mathrm{CN}$ to $4.8 \mathrm{CN}$ at a $\mathrm{CN}$ level of $56 \mathrm{CN}$ [11]. The poor precision, high capital cost, large fuel sample quantity, and long testing time of this test method have been problematic for the petroleum industries.

\subsubsection{Ignition Quality Tester (IQT ${ }^{\mathrm{TM}}$ ) ASTM-D6890}

Advanced Engine Technology Ltd. (AET, Ottawa ON) has developed a novel diesel fuel Ignition Quality Tester (IQT ${ }^{\mathrm{TM}}$ - ASTM D6890 [1]) that permits more reproducible measurements of the ignition delay of diesel fuels. The technology is based on a small constant volume combustion based apparatus initially developed by Southwest Research Institute [12]. A correlation equation was experimentally generated in 2002 assigning a derived cetane number (DCN) to the ignition delay (ID in $m s$ ) of a fuel sample. This DCN is found by correlating the ignition delay from the D6890 test method to the CN determined from the D613 method as:

$$
D C N=4.460+\frac{186.6}{I D}
$$


Since the introduction of the IQT ${ }^{\mathrm{TM}}$, more than 145 units have been commissioned worldwide. The accuracy of the IQT ${ }^{\mathrm{TM}}$ allows refineries to blend closer to the legislated minimum CN. The IQT ${ }^{\mathrm{TM}}$ thus saves on 'cetane giveaway' which has resulted in substantial savings for refineries. Precision and repeatability are paramount to CN determination as refineries want the most precise instrument available in the market. More accurate and repeatable CN measurements provide considerable economic benefits for refineries in terms of savings of additives, which otherwise would have to be included in order to maintain the ignition quality level. Further improvements to the precision of the IQT ${ }^{\mathrm{TM}}$ would translate into an important competitive advantage for the IQT ${ }^{\mathrm{TM}}$ as existing users and potential customers strive to further reduce their production costs.

The IQT ${ }^{\mathrm{TM}}$ is also a well established reference research instrument. Many research institutions in the fuel and combustion field use the IQT ${ }^{\mathrm{TM}}$ for parametric studies to characterize the ignition delay of novel fuels. The IQT ${ }^{\mathrm{TM}}$ is featured in academic papers published by research institutions such as Southwest Research Institute, Wayne State University, ExxonMobile Research and Engineering and Sasol Oil [13] [14]. The importance of the IQT $\mathrm{T}^{\mathrm{TM}}$ in this sector is growing rapidly. The cross-correlation between the IQT ${ }^{\mathrm{TM}}$ (ASTM D6890) and the CFR Engine (ASTM D613) will not likely hold for some new novel biomass derived fuels [14]. The CN of novel biomass derived fuels measured using the CFR engine has been shown to mispredict auto-ignition characteristics when compared to actual engine tests. [15]. The IQT ${ }^{\mathrm{TM}}$ has the potential of experimentally validating the combustion kinetic models of these novel fuels. The temperature, pressure, oxygen content and the amount of mass injected are all known and controllable variables within the IQT ${ }^{\mathrm{TM}}$ which makes model implication simple. Bogin et al. [14] modelled the fuel spray and the combustion process of $n$-heptane within the IQT ${ }^{\mathrm{TM}}$ in 2009. Sasol Advanced Fuels Laboratory in South Africa also 
conducted a similar study on the combustion model of n-heptane within the IQT ${ }^{\mathrm{TM}}$ in 2007 [13]. Both their findings concluded a small discrepancy between the experimental and modelled ignition delay. The discrepancy can be partly attributed to the difference between the experimental and modelled spray characteristics. The lack of spray characteristics of the IQT ${ }^{\mathrm{TM}}$ creates uncertainty in the physical ignition delay of their model. Physical ignition delay is the time required for the injected fuel to break up, evaporate and entrain air for mixing. Similarly, the chemical ignition delay is the time required for combustion kinetics to sustain combustion. Validating experimental results from the $\mathrm{IQT}^{\mathrm{TM}}$ to chemical kinetic models without an appropriate physical characteristic of the injection spray is difficult. Spray characteristics of the S-type pintle nozzle, used within the IQT ${ }^{\mathrm{TM}}$, in literature is limited to only front light photography and line of sight laser diffraction techniques dating back to the early 1980's [16]. A better spray characterization approach is needed for further development of combustion models using the $\mathrm{IQT}^{\mathrm{TM}}$.

\subsection{Previous Work Completed}

Previous work completed in 2009 by M. Karakolis revealed that most of the bias error of the IQT ${ }^{\mathrm{TM}}$ community is due to different injection system characteristics [17]. Understanding the behavior of the injection spray of the IQT ${ }^{\mathrm{TM}}$ is necessary in order to evaluate how the reproducibility can further be improved. Over the years, many researchers have successfully characterized various types of injection systems using various optical measurement techniques, however, the IQT ${ }^{\mathrm{TM}}$ has a unique patented fuel injection system where the spray characteristics of this injection system are still not fully understood. 


\subsection{Thesis Objective}

The objective of this thesis is to characterize the velocity and droplet size of the $\mathrm{IQT}^{\mathrm{TM}}$ fuel injection spray to better understand the atomization process and to investigate the effect of different IQT $\mathrm{T}^{\mathrm{TM}}$ injection parameters on atomization performance. Phase Doppler Anemometry (PDA) is used to investigate the size and velocity characteristics of droplets from the IQT ${ }^{\mathrm{TM}}$ 's injection spray into atmospheric ambient air. This technique is widely used in characterizing aerosols, diesel sprays and other types of jets. The high temporal resolution of this technique enables the investigation of time resolved velocity and diameter. The droplet count also indicates the size distribution as a function of time and spatial position. This information is then used to develop correlated spray characteristic equations valid at the nozzle outlet. This will be of significant importance to researchers modelling the IQT $\mathrm{T}^{\mathrm{TM}}$, allowing the implementation of a valid spray characteristic inlet condition, which is necessary for kinetic model validation. This information will also help AET to better understand the spray characteristics and the effect of changing parameters such as the injection pressure and the amount of mass injected on the atomization performance within the $\mathrm{IQT}^{\mathrm{TM}}$.

\subsection{Outline}

Chapter 2: Background and Literature Review: This chapter covers the $\mathrm{IQT}^{\mathrm{TM}}$ operational procedures for the determination of the derived cetane number (DCN). Diesel spray breakup theory and various fuel spray characterization methods are also reviewed in this section.

Chapter 4: Experimental Apparatus: The experimental apparatus is covered in detail in this chapter. The complete injection system of the $\mathrm{IQT}^{\mathrm{TM}}$, the injection 
sequence, all electronics associated with the IQT ${ }^{\mathrm{TM}}$, including the trigger circuit synchronizing the fuel spray to the measurement system, are described.

Chapter 3: Laser and Phase Doppler Anemometry: This chapter focuses on the theory behind laser interferometric measurements such as Laser and Phase Doppler Anemometry. Detailed information on the physical principles of the measurement is presented to the reader. This information is necessary in order to understand the decisions made during the selection of measurement system parameters in Chapter 5 .

Chapter 5: Measurement Parameter Selection: In this chapter, the scattering properties of droplets are explained for the purpose of understanding how different measurement system parameters can affect experimental results. Experiments were conducted in order to optimize the laser power, the system's high voltage, the signal to noise ratio, and the injection sample size for this study. This section also introduces the measurement uncertainty for both diameter and velocity statistics.

Chapter 6: Results In this chapter, the data analysis procedure and different derived spray parameters are introduced. Followed by the IQT ${ }^{\mathrm{TM}}$ spray characteristic results for the following test conditions:

- Standard IQT $T^{T M}$ operation: Included in this analysis are the time resolved front light visualizations using a high speed camera and averaged PDA results for the main injection. The PDA results are further investigated in a time resolved manner in order to generate the velocity spray profile as a function of time.

- The effect of mass injected: In this analysis, the averaged PDA results and the injector needle trace are compared to the standard IQT ${ }^{\mathrm{TM}}$ operation. 
- The effect of the injection pressure: Similarly, the averaged PDA results and injector needle trace are compared to the standard IQT ${ }^{\mathrm{TM}}$ operation.

- The effect of the ambient pressure on penetration rate: A custom optical chamber is fitted to the experimental apparatus. The spray penetration rate is investigated as a function of the ambient pressure using front light visualization. The results of the penetration rate is compared to previous work completed by different authors.

- The effect of the ambient pressure on the droplet size statistics: Although no PDA measurements were performed at different ambient pressures, the droplet size statistical behaviour as a function of ambient pressure is reviewed for sake of completeness.

- Backtracking algorithm: A backtracking algorithm is introduced. This algorithm is used to backtrack velocity and diameter statistics to the nozzle outlet under atmospheric ambient condition. The results of this algorithm provides modellers with the velocity, diameter, and count profiles at the IQT ${ }^{\mathrm{TM}}$ nozzle outlet. Spray conditions at the nozzle outlet are believed to be similar despite the ambient pressure given that the pressure differential across the nozzle remains constant.

Chapter 7: Conclusion A summary of this study is presented in this chapter. In addition, future work considerations and recommendations are presented in order to improve the overall unit to unit variability of the IQT ${ }^{\mathrm{TM}}$. 


\section{Chapter 2}

\section{Background and Literature Review}

\subsection{Ignition Quality Tester IQT $^{\mathrm{TM}}$}

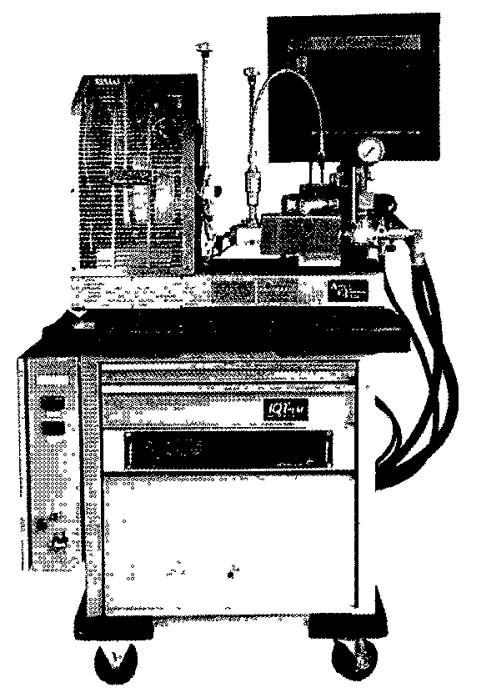

Figure 2.1: $\mathrm{IQT}^{\mathrm{TM}}$ instrument

Although not directly used in the current thesis, the whole IQT ${ }^{\mathrm{TM}}$ system and operation is briefly described here for the sake of completeness. The IQT ${ }^{\mathrm{TM}}$ is a constant volume combustion chamber apparatus developed to measure the ignition delay of diesel fuels. The IQT ${ }^{\mathrm{TM}}$ functions by measuring the ignition delay of fuels, which is then used to calculate a derived cetane number (DCN) using a cross correlation with 
the CFR Engine (ASTM D613) [11]. The IQT ${ }^{\mathrm{TM}}$ schematic is represented in Figure 2.2. The volume of the stainless steel combustion chamber is $0.213 \mathrm{~L}$. The chamber is embedded with nine electrical cartridge heaters used to heat the walls and contents of the chamber. Three thermocouples monitor the chamber temperature; two internal thermocouples measure the front and back chamber temperatures and the third thermocouple measures the chamber skin temperature. The thermocouple on the chamber skin provides feedback for the heaters to ensure that the chamber stays at a relatively constant set point temperature. This temperature is set based on an n-heptane calibration procedure as per ASTM D6890 [1]. 


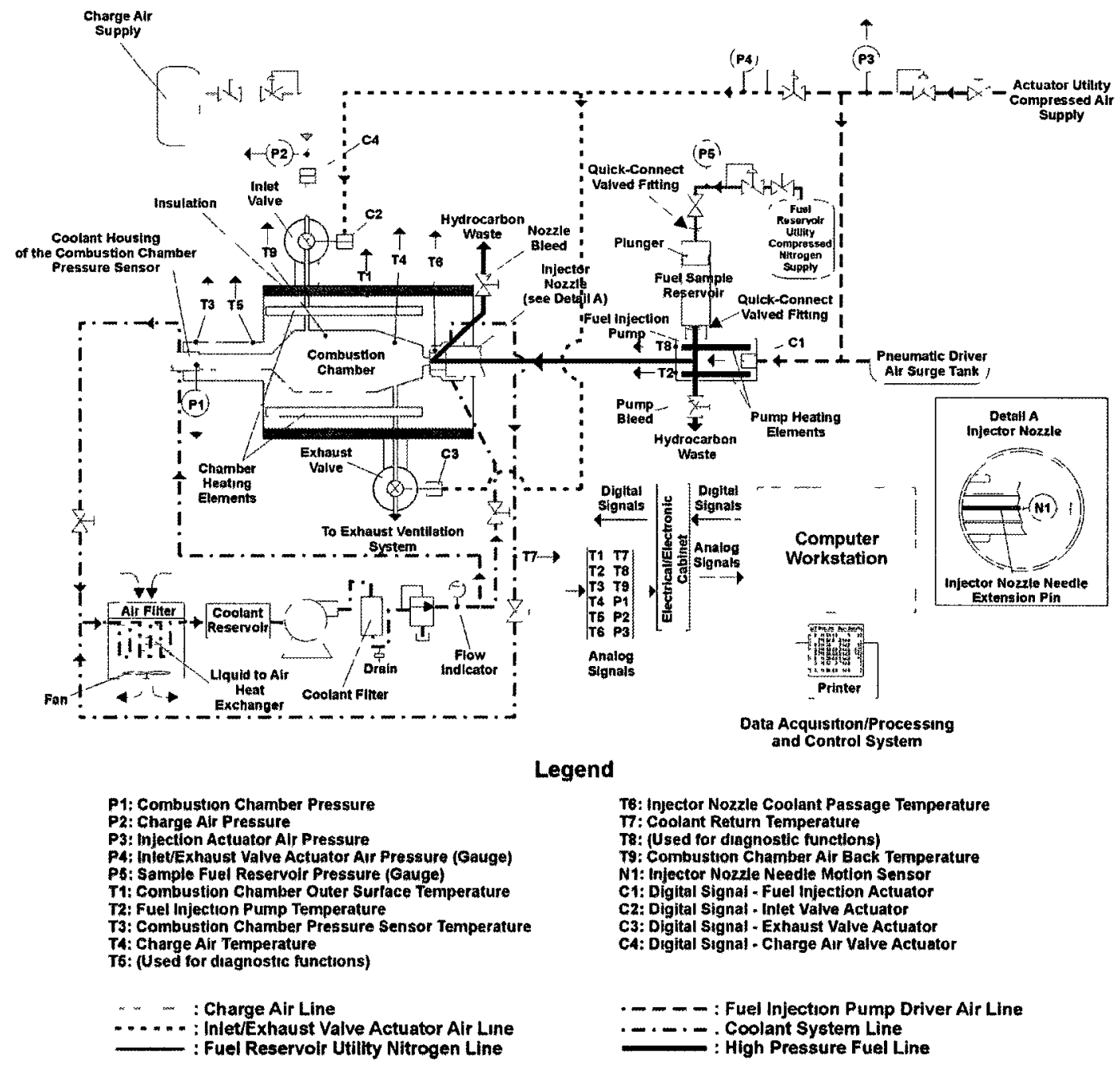

Figure 2.2: $\mathrm{IQT}^{\mathrm{TM}}$ schematic [1] 
There are two pneumatically actuated valves controlling the air flow to the chamber. Air is introduced into the chamber at a pressure of 21.1 bar. The fuel injection nozzle and holder are located at one side of the chamber. A proximity sensor mounted within the nozzle holder measures the movement of the fuel injection nozzle's needle valve as fuel is injected into the chamber. A piezoelectric pressure transducer located on the opposite side of the chamber monitors the pressure during the combustion process.

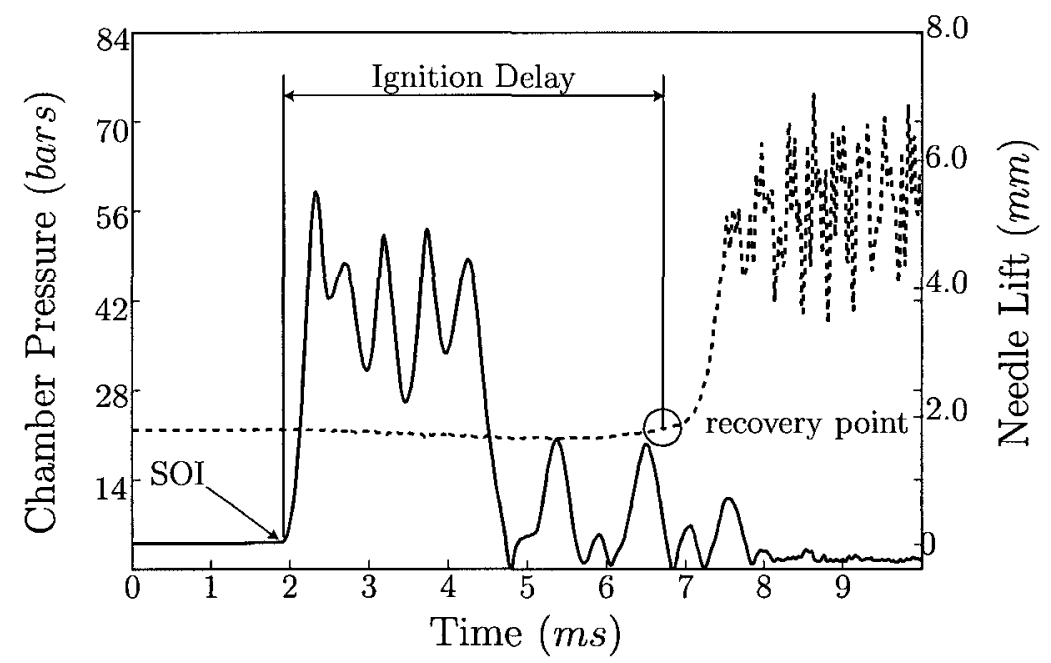

Figure 2.3: IQT $^{\mathrm{TM}}$ Ignition delay determination ASTM D6890 [2] [1]. Solid line represents the needle lift. Dashed line represents the chamber pressure.

The IQT ${ }^{\mathrm{TM}}$ measures the ignition delay as illustrated in Figure 2.3. The needle valve trace (solid line) is used to determine the start of injection (SOI) while the combustion chamber pressure trace (dashed line) provides an indication of the start of combustion within the chamber. The chamber pressure decreases after the SOI due to evaporative cooling of the vaporizing droplets injected. The pressure in the combustion chamber increases very rapidly once sustained combustion begins to occur. The ignition delay is measured from the SOI to the pressure recovery point (i.e. initial chamber pressure) [14]. 
The entire IQT ${ }^{\mathrm{TM}}$ test sequence requires 17 minutes for completion and consists of 15 warm-up combustion cycles. These initial cycles are necessary to reach thermal equilibrium. The test is followed by 32 main combustion cycles which are used to determine the average ignition delay and standard deviation of the measurement. The chamber is purged with a constant stream of air after each combustion event and then refilled with pressurized air having an $\mathrm{O}_{2}$ concentration of $20.9 \%$. The patented compressed-air-actuated fuel injection system is composed of a specialized barrel/plunger and an inward opening S-type delayed pintle-type nozzle, which will be covered in detail in Section 4.1.2. The pintle injection nozzle was selected for its satisfactory hollow spray cone atomizing characteristics with a broad range of different diesel fuels with varying fuel properties [17].

\section{$2.2 \quad$ Diesel Spray Breakup}

Diesel spray structures can be represented by 4 processes. First the primary breakup mechanisms at the nozzle outlet, followed by the secondary breakup, followed by the evaporation and mixing of droplets with the surrounding gas, and the chemical reaction of the mixture.

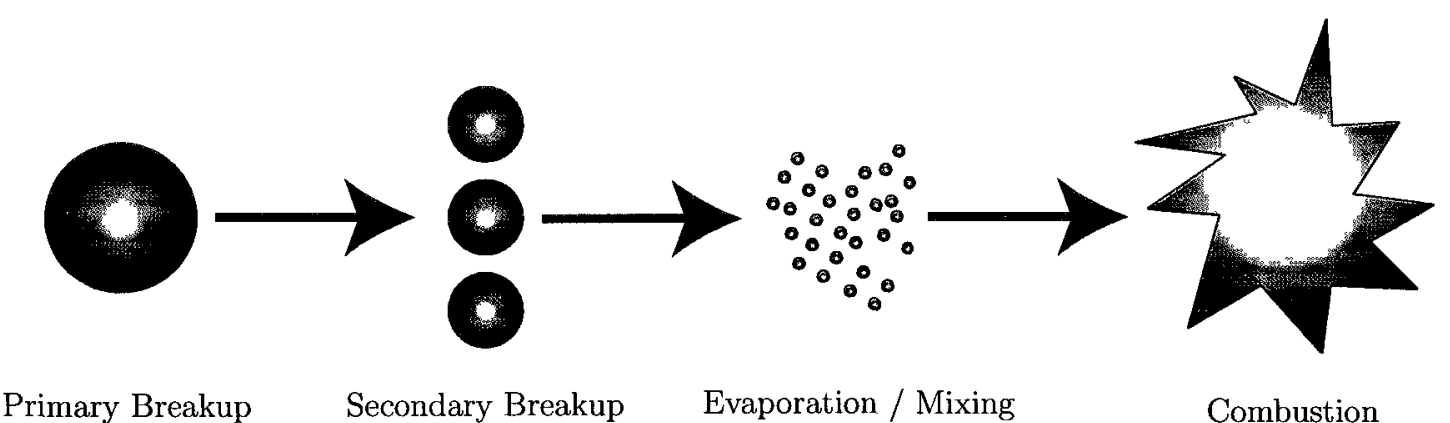

Figure 2.4: Diesel spray combustion 


\subsubsection{Primary Breakup}

The primary breakup is one of the most important steps of the injection process. The main breakup mechanism is caused by the cavitation of the fuel passing through the nozzle hole. The high fuel line pressure causes voids in the liquid core at the exit of the nozzle [18]. The bursting and spontaneous collapse of these vapor cavities disintegrate the liquid core at the nozzle exit by promoting rapid instability growth of the liquid core [19]. The cavitation also promotes instability from the pressure wave generated by the implosion of voids [20]. The liquid core is sheared into both ligaments and large droplets. Initially, it was believed that the liquid core existed some distance away from the nozzle exit. However, modern fluid measurement techniques such as back lit imaging are now able to measure the core of the spray at the nozzle outlet [19], [21]. The liquid core is found to be completely atomized after only a few nozzle diameters downstream [22]. The liquid fuel at the nozzle exit of the $\mathrm{IQT}^{\mathrm{TM}}$ is expected to behave as a cavitating flow based on work completed by Chaves et al. [3]. Figure 2.5 demonstrates how the IQT ${ }^{\mathrm{TM}}$ injection is expected to behave in the cavitation region.

\subsubsection{Secondary Breakup}

The secondary breakup of ligaments and large droplets is attributed to the aerodynamic forces causing distortion of the liquid fuel [23]. The fuel viscosity and surface tension resist the distortion to the point where the aerodynamic forces are too great and droplet breakdown occurs. The Weber number quantifies the onset of droplet breakdown which is governed by the ratio of aerodynamic forces acting on the droplet compared to its surface tension [24]. The gas Weber number is represented by:

$$
W e_{g}=\frac{\rho_{g a s} U_{r e l}^{2}}{2 \sigma / D}
$$




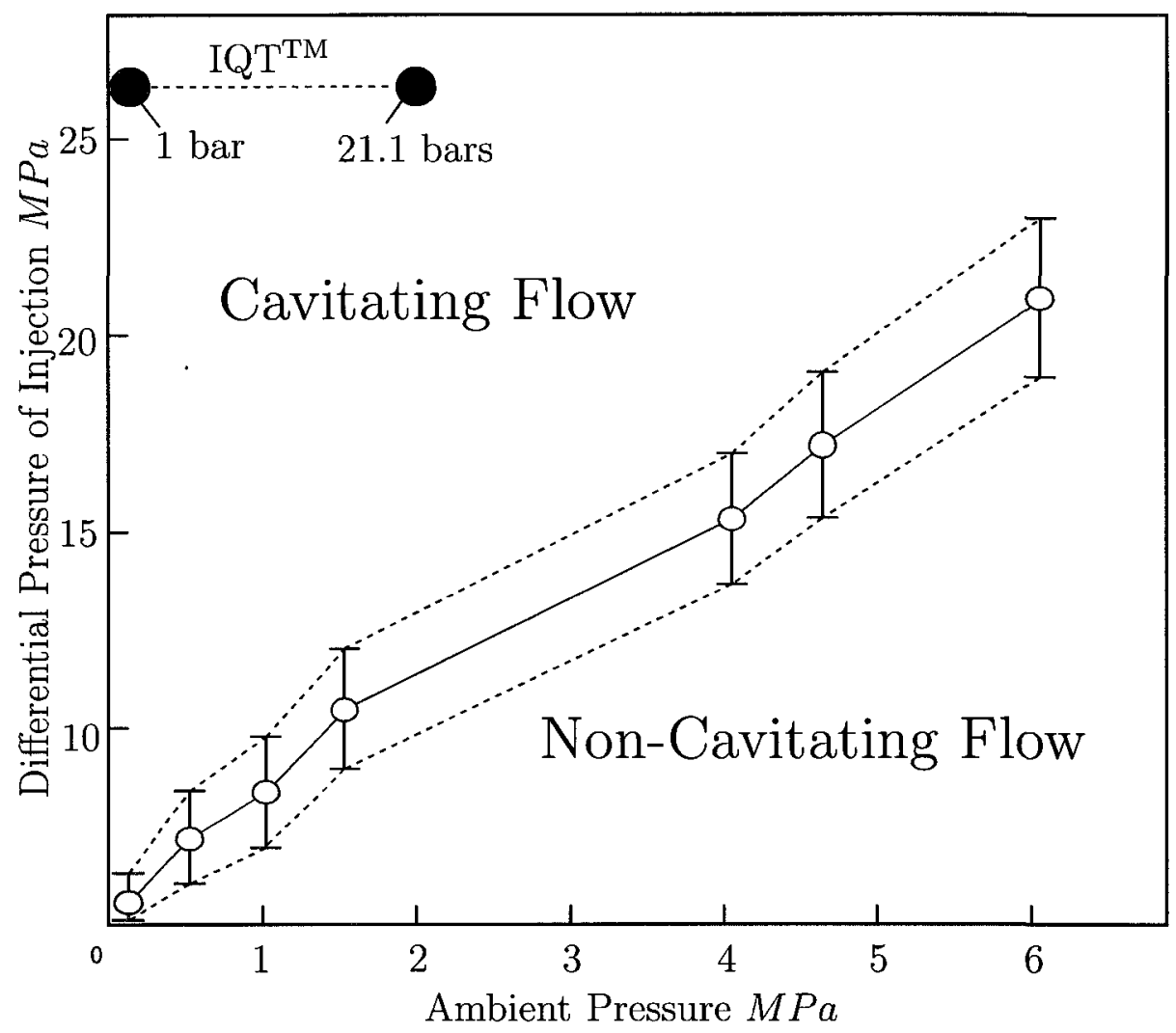

Figure 2.5: Regime of cavitating and non-cavitating flow through Diesel nozzles [3]

where, $\rho_{\text {gas }}$ is the surrounding gas density, $U_{r e l}$ is the droplet relative velocity, $\sigma$ is the liquid surface tension, and $D$ is the droplet diameter.

The breakup phenomenon is governed by the ratio of fuel inertia to the internal viscous force $(R e)$, the ratio of distortion forces to the surface tension $\left(W e_{l}\right)$, and the ratio of internal liquid viscous force to the surface tension $(Z)$ [25]. These three nondimensional parameters play an important role in determining the critical Weber number at which droplet breakup occurs. The Ohnesonge number $(Z)$ which can be represented by $Z=\sqrt{W e_{l}} / R e_{1}$, governs the critical gas Weber number $W e_{g}$ at which secondary breakup occurs. Flows with a $Z$ number smaller than 0.1 will experience secondary breakup at $W e_{\text {critical }}$ between 6 and 12. While the critical Weber number $\left(W e_{\text {critical }}\right.$ ) of flows with $Z \gg 0.1$ is approximately $\simeq Z^{2}[26]$. 
There are a few secondary breakup regimes suggested in literature. The bag breakup, which occurs at lower Weber numbers slightly above the $W e_{\text {critical }}$ number and catastrophic breakup, which happens at $W e_{g}>0.5 \sqrt{R e_{d}}[27]$. Figure 2.6 demonstrates the differences between the two secondary breakup regimes. All above calculations were completed using No.2 diesel oil properties available in Appendix A.
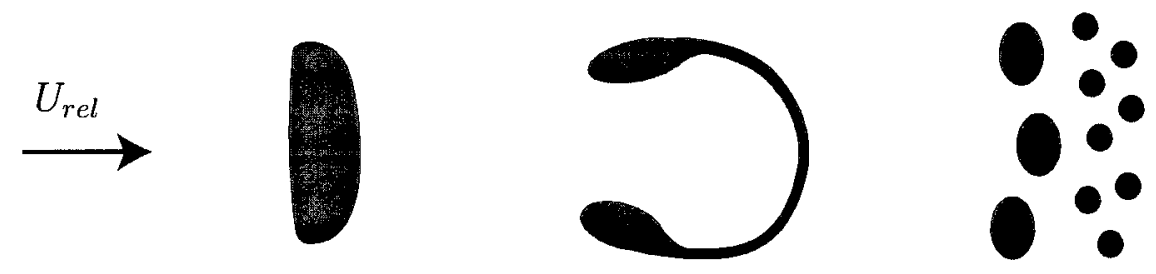

Bag Breakup
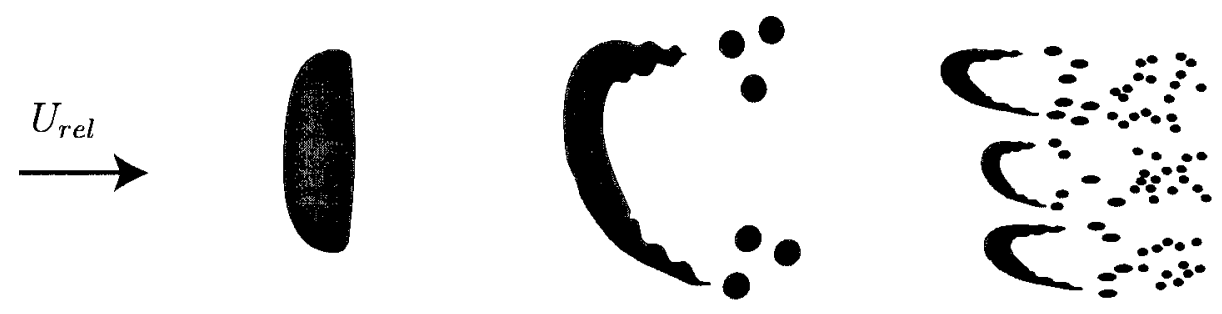

Catastrophic Breakup

Figure 2.6: Droplet secondary breakup regimes. Adapted from: [4]

\subsubsection{Evaporation / Mixing}

The evaporation is promoted by heat and mass transfer from the droplet to the surrounding gas. The liquid droplet surface has a specific fuel vapor mass fraction depending on the initial liquid fuel temperature. This mass fraction produces a concentration gradient at the droplet surface promoting diffusion from the droplet towards the surrounding gas. The Spalding number $(B)$ is a non dimensional mass 
fraction gradient represented by:

$$
B=\frac{Y_{R}-Y_{\infty}}{1-Y_{R}}
$$

where $Y_{R}$ and $Y_{\infty}$ are the mass fraction of fuel at the droplet surface and in the surrounding gas, respectively. The mass fraction at the droplet surface can be found when the vapour pressure of the fuel is known. The Antoine equation is a reasonably accurate vapor pressure equation:

$$
P_{V}=\exp \left(A-\frac{B}{T+C}\right)
$$

where the coefficient $\mathrm{A}, \mathrm{B}$ and $\mathrm{C}$ are specific to the fuel [28].

$$
G=\frac{\rho D_{A B}}{R} \ln (1+B)
$$

The mass flux from the droplet can be found knowing the diffusivity of the liquid fuel $D_{A B}$ to the surroundings using Eq. (2.4). The change in the droplet mass can then be represented by Eq. (2.5) where the loss of mass from the droplet equals the mass flux times the surface area. For the ideal case of perfectly spherical droplets, equation Eq. (2.5) simplifies to:

$$
\frac{d m}{d t}=4 \pi \rho_{L} R^{2} \frac{d R}{d t}=-4 \pi R^{2} G
$$

These equations indicate that the droplet surface area decreases linearly as a function of time. This is known as the $d^{2}$ law of droplet evaporation [29].

The droplet life time, represented by $t_{\text {evap }}$, is dependent on the evaporation driving constant $K_{V}$. Eq. (2.7) underestimates the droplet lifetime as the transient heating period of the liquid droplet from the fuel reservoir temperature to the chamber temperature is ignored.

$$
K_{V}=8 \frac{\rho_{a i r} D}{\rho_{L}} \ln (1+B)
$$




$$
d=d_{o}-\sqrt{\Delta t K_{V}} \rightarrow t_{\text {evap }}=\frac{d_{o}^{2}}{K_{V}}
$$

This analysis consists of mass transfer mainly by diffusion. In a diesel spray, large droplet velocities accelerate the evaporation of droplets by transferring more efficiently fuel vapor at the droplet surface to the surrounding gas. This additional convective heat and mass transfer results from large relative velocities between the droplet and the stagnant air. This additional convection term greatly increases the droplet evaporation constant $K_{V}$.

\subsection{Diesel Spray Penetration}

Diesel spray penetration is an important spray characteristic. Spray penetration is the maximum attainable droplet distance from the nozzle tip. For sprays in atmospheric pressure and temperature, this distance can be quite long since mass transfer driving forces are low. However, in engine like conditions, this distance can be of the orders of only 100 nozzle diameters. Spray penetration has been the most studied feature of diesel sprays using front light visualization techniques. Adequate penetration is required for proper mixing of the fuel and air to obtain proper local equivalence ratios [20]. The penetration distance is affected by the charged air temperature and density. The Spalding transfer number increases with the charged air temperature. This results in larger mass flux from the droplet surface as discussed in Section 2.2.3 resulting in shorter droplet life time and penetration distance. The density has a similar effect on penetration where an increase in gas density results in larger Weber number, promoting droplet breakup and a lower penetration distance [30]. Penetration distance is also a function of the chamber pressure as the drag force increases linearly with the ambient gas density. 


\subsection{Measurement techniques for sprays}

Various flow visualization techniques have played a very important role in understanding different fluid atomization problems for different applications. Using high speed digital CCD cameras, laser diagnostics and advanced image analysis, it is possible to derive qualitative and quantitative information about the atomization process. A large variety of flow visualization techniques are available for characterizing sprays.

\subsubsection{Planar Mie Scattering}

The planar Mie scattering technique using laser sheet illumination (LSI) is one of the most common flow visualization techniques used by researchers (e.g., [31], [32], [33]). The planar Mie scattering technique can generate instantaneous as well as average images of the spray. This technique has been extensively used due to its simplicity. The concept of LSI is to illuminate a seeded flow using a sheet of laser light while capturing the scattered light of the seeded particulates using a camera. The spray parameters derived from LSI analysis are the cone angle and the solidity. The spray solidity is the ratio of droplet to empty space within the cross section of the spray [34]. The laser sheet, used to illuminate the plane of interest across the spray, can be produced in two different ways.

1. The conventional way to produce a sheet of light is to pass the incident beam through a plano-convex cylindrical lens. The light intensity profile of the laser sheet produced in this manner is Gaussian distributed centered on the lens axis [35]. The illumination plane and the intensity profile are illustrated in Figure 2.7(a).

2. As the laser sheet, generated by the above conventional method, is not uniformly distributed across the region of interest, an improvement can be made to the 
LSI system by replacing the cylindrical lens with a rotating mirror. Using this method allows the generation of a uniform laser sheet across the plane of interest and avoids the complex image correction associated with the Gaussian distributed sheet. Figure 2.7(b) demonstrates the generation of the dynamic laser sheet using a rotating mirror.

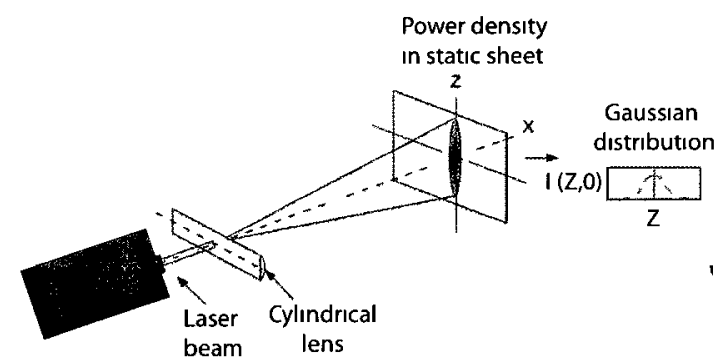

(a) Static laser sheet from cylindrical lens

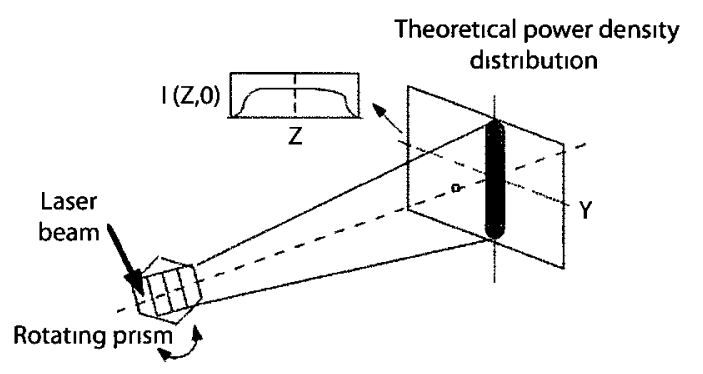

(b) Dynamic laser sheet from rotating prism

Figure 2.7: Techniques for converting a laser beam into a sheet

\subsubsection{Back lit imaging (Shadowgraphy)}

Another technique that is very useful for microscopic investigation of the ligament breakup in atomizing sprays is back lit imaging. This technique utilizes a strobe or flash lamp synchronized to a CCD digital camera. The principle of back lit imaging is to capture the shadow of the ligaments at the atomizer outlet [19], [21]. The strobe lamp flashes for a short period of time. This short exposure freezes the ligaments which are then imaged on the camera. This technique is more effective in freezing ligaments and droplets compared to the planar Mie method. In order to freeze frames using the LSI technique, a short camera shutter time is required. In order to get a good image under short shutter time conditions, an extensive amount of incident light power is required. 


\subsubsection{Particle Image Velocimetry (PIV)}

The measurement principle of PIV is to correlate the movement of seeded particle clusters between two instantaneous images. A vector can then be associated to each cluster knowing the time between the frames and a calibration distance from the image generating a 2-D vector field. PIV generally utilizes a light sheet generated from a Nd:YAG ${ }^{1}$ laser to illuminate the seeded flow. This laser produces pulses of high energy which can be controlled using a Q-switch to trim off the Gaussian distribution of the pulse [36]. The pulse duration of these lasers are usually between $100 \mathrm{ps}-10 \mathrm{~ns}$. PIV requires a large amount of seed particles for appropriate measurements. For diesel spray applications, performing PIV measurements using only the fuel droplets as the seed is difficult. In diesel sprays, PIV is usually performed using a seeded fluorescent dye in suspension in a quiescent pressure vessel [37] [38]. The fluorescent seeds are then imaged using a bandpass filter on the camera lens. The vector field now represents the ambient combustion air entrainment through the fuel spray which is important in understanding the fuel/air mixing process [39].

\subsubsection{Laser Diffraction and Laser Interferometry}

Quantitative spray characteristics are of significant importance for the atomization and spray sector. The most important characteristics of evaporative sprays are the velocity and the size of droplets as the design of combustion chambers, gasifiers and burners depends on the droplet trajectories, penetration rates and residence times. More importantly, the symmetry of the spray is paramount as an asymmetrical mass delivery from atomizers results in significantly different local equivalence ratios. These measurement techniques include laser diffraction and laser interferometry. The laser diffraction method interrogates the flow field in a line of sight using spatial sampling

\footnotetext{
${ }^{1}$ Solid state lasers containing rare earth neodymium $\left(\mathrm{Nd}^{+3}\right)$ incorporated into a crystal of yttrium aluminum garnet [36]
} 
while the interferometric technique uses temporal sampling at a point measurement (flux sampling). The disadvantage of the laser diffraction method is the spatial resolution of the measurement when compared to the interferometric technique [40]. A significant advantage of the interferometric technique is the simultaneous measurement of the size of droplets and their respective velocities. The interferometric technique is the measurement technique implemented in this study and will be covered in detail in Chapters 3 and 5. 


\section{Chapter 3}

\section{Laser and Phase Doppler Anemometry}

The first successful PDA fluid measurement experiment was presented in 1975 by Durst and Zaré [41] where they successfully determined simultaneously the velocity and size distribution of suspended fluid particles. The PDA technique known today is far more advanced and precise than the original Phase Doppler measurements performed. The largest contributions to the technique came in the mid 80's where the introduction of three photo detectors allowed measurements of small sub-micron particles [42] [43]. Initially, this technique was used for low particle proximities as characterization of dense sprays such as diesel fuel injectors which was impossible due to the incident beam attenuation through the dense droplet field. With the rapid evolution of laser technology, laser sources of very high power are available and characterization of dense sprays can be performed.

\subsection{Measurement Principle}

Phase Doppler Anemometry is often mistaken with Laser Doppler Anemometry however they are different in many ways. Both modern Phase and Laser Doppler Anemometry are point measurement techniques. The measurement volume is formed 


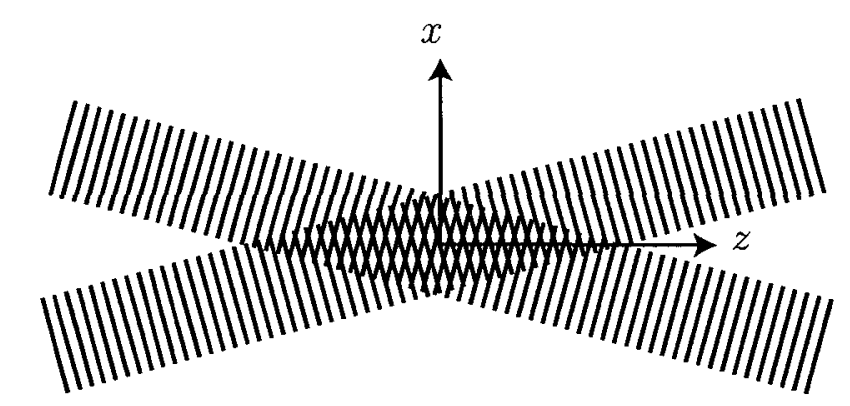

Figure 3.1: Measurement volume from intersecting coherent laser beams

by the intersection of two coherent monochromatic light beams as illustrated in Figure 3.1. The particle velocity and size statistics are contained in the scattered field of light when particles cross the measurement volume due to the Doppler effect [6].

\subsubsection{Laser Doppler Anemometry}

Measuring particle velocities using theory from the Doppler effect seems reasonably simple. However, the hardware limitation prevents us from simply measure scattered light from a single monochromatic coherent light source. Figure 3.2 demonstrates how the Doppler effect is used to measure particle velocity. When the incident light beam, which has a wavelength of $\lambda_{b}$ and velocity $e_{b}$, collides with the moving particle with velocity $V_{p}$, scattered light is emitted in all directions. If a photo detector is positioned at angle $\theta_{r}$, the emitted scattered light from the particle to the photo detector will have a velocity of $\mathbf{e}_{p r}$. This emitted light produces a sinusoidal signal with frequency $f_{r}$ found using the following equation:

$$
f_{r}=f_{b}+\frac{\mathbf{V}_{p} \cdot\left(\mathbf{e}_{p r}-\mathbf{e}_{b}\right)}{\lambda_{b}}
$$

The problem with this simple LDA arrangement is that for typical flow situations, the shifted frequency $f_{r}$ is on the order of $10^{14} \mathrm{~Hz}$. This shifted frequency is practically impossible to measure using conventional photo detectors [6]. The first successful LDA experiment dates back to 1964 where Cummins et al. measured the velocity of 


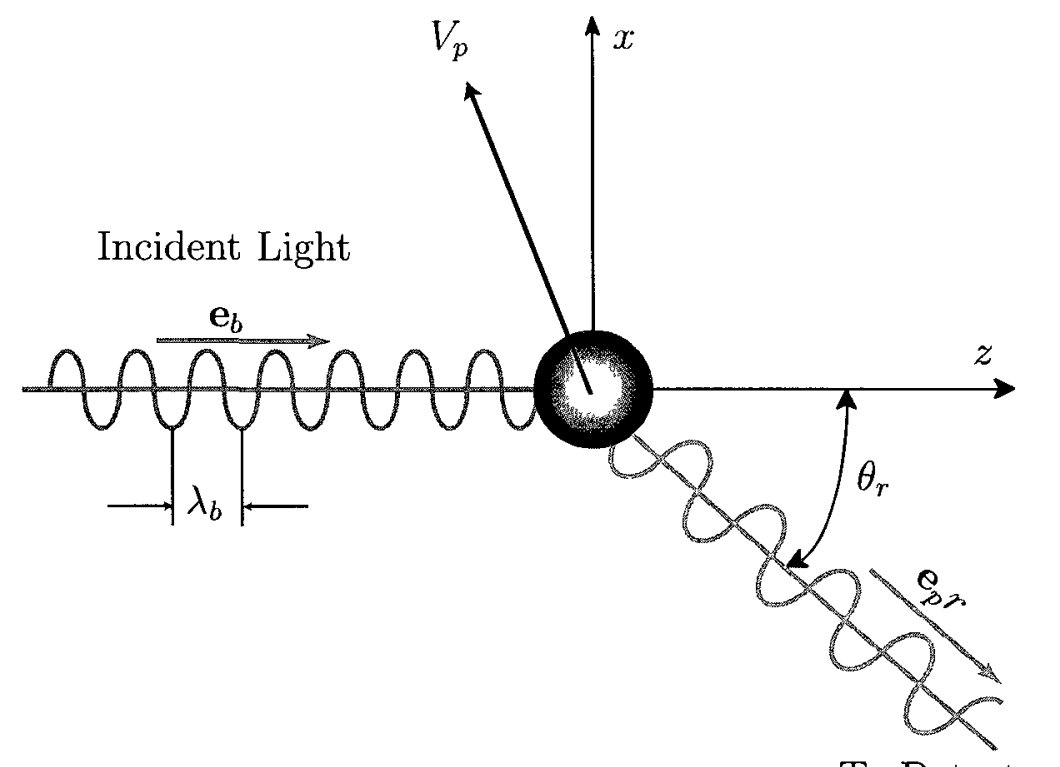

To Detector

Figure 3.2: Single beam LDA principle

polystyrene spheres using a reference beam technique [44]. This featured only one light beam as illustrated in Figure 3.2, however, the detector was provided with the incident light wave and by a process known as heterodyne, the Doppler shift was measurable. Heterodyne is the combination of two frequencies which produces a beat frequency. Both the reference beam and the scattered light produce frequencies that are outside the scope of measurement. When these two signals are combined, they produce a beat frequency which now lies within the measurement scope of the photo detector.

Today, modern LDA systems use two intersecting incident beams in order to resolve the above issue. Figure 3.3 illustrates a modern LDA arrangement where $e_{1}$ and $e_{2}$ are the incident coherent beam producing an intersection angle of $\phi$. Using this configuration, the Doppler frequency $f_{D}$ is now independent of the detector position [6]. Eq.(3.2) demonstrates how the $x$ component of velocity can be found from the Doppler frequency of scattered light independent of detector position. The Doppler 
frequency is also known as the beat frequency or the Doppler burst where $f_{D}$ is a combination of $f_{1}$ and $f_{2}$. This $f_{D}$ now lies in a reasonable frequency range that is easily detectable by photo detectors without the need of a reference beam [6].
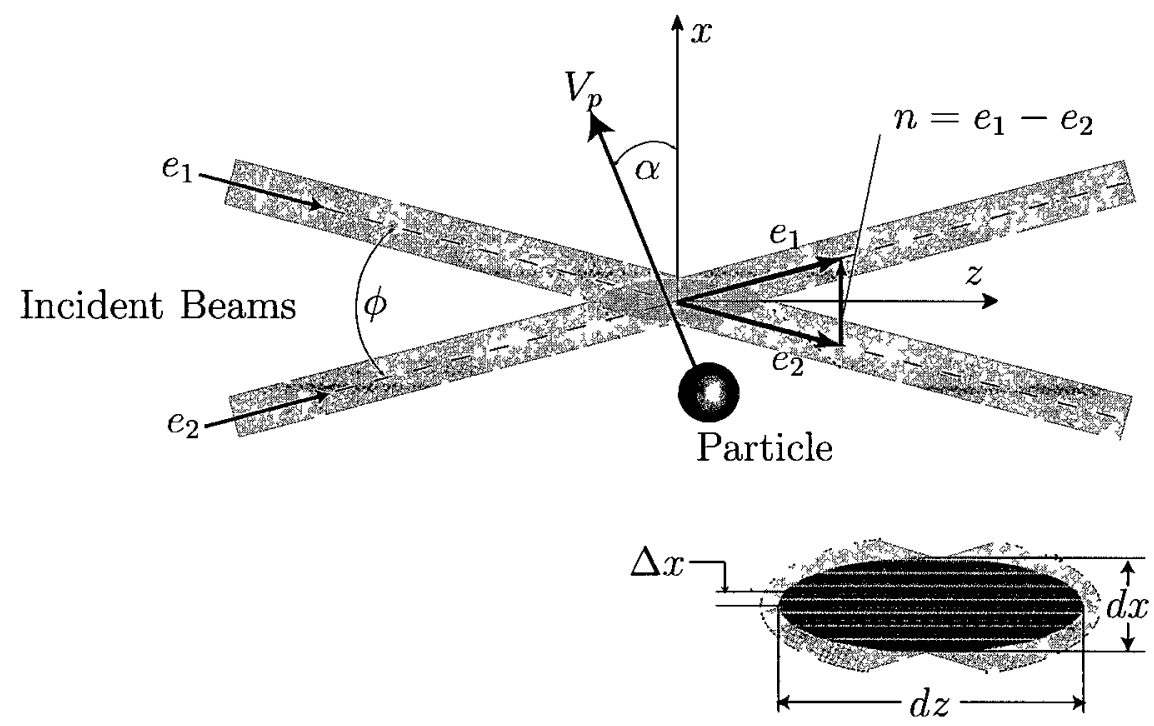

Figure 3.3: Modern two beam LDA configuration

$$
f_{D}=\frac{2 \sin \left(\frac{\phi}{2}\right)}{\lambda_{b}}\left|V_{p}\right| \cos \alpha=\frac{2 \sin \left(\frac{\phi}{2}\right)}{\lambda_{b}} v_{p \perp}
$$

Another way of explaining LDA using the more popular approach, is the interference fringe model as illustrated in Figure 3.1. The intersection of the two beams produces an intensity profile in the $x$ direction which is function of the parameters in Figure 3.3. The intensity profile of the measurement volume is represented by Eq: (3.3).

$$
I=\varepsilon c E_{o}\left[1+\cos \left(2 \pi \frac{2 \sin \frac{\phi}{2}}{\lambda_{b}}\right)\right]
$$

If all optical parameters from Figure 3.3 are known, the fringe spacing is found using Eq: (3.4) 


$$
\Delta x=\frac{\lambda_{b}}{2 \sin \frac{\phi}{2}}
$$

From geometry, the size of the measurement volume can be approximated knowing the incident beam diameter $d_{b}$ and the intersecting angle $\phi$ as:

$$
\delta x=\frac{d_{b}}{\sin \left(\frac{\phi}{2}\right)} \quad \delta z=\frac{d_{b}}{\cos \left(\frac{\phi}{2}\right)} \quad \delta y \simeq d_{b}
$$

Knowing the burst frequency $f_{D}$ from the photo detector signal and the fringe spacing $\Delta x$, the perpendicular velocity $V_{\perp}$ can now be found using the following equation:

$$
V_{p \perp}=V_{p x}=f_{D} \Delta x
$$

A disadvantage of using two beams as described so far, is the fact that a particle crossing the measurement volume from bottom to top with velocity $+v_{x}$ produces the identical Doppler burst signal as a particle crossing from top to bottom with velocity $-v_{x}$ if $\left|+v_{x}\right|=\left|-v_{x}\right|$. To handle the ambiguity of particle direction, a Bragg cell is added to one of the incident beams. The Bragg cell is composed of an electro mechanical transducer driven by an oscillator and shifts the frequency of beam $e_{2}$ by $f_{o}$. This frequency shift ensures that a particle with velocity $-v_{x}$ introduces a distinguishable Doppler burst. The shifted Doppler burst frequency is now: [6]

$$
f_{D}=\left|f_{o}+\frac{2 \sin (\phi / 2)}{\lambda} v_{x}\right|
$$

\subsubsection{Phase Doppler Anemometry}

Phase Doppler Anemometry has the same physical measurement principle as LDA with a few minor differences that allow precise measurement of the diameter statistics of particles crossing the measurement volume. PDA uses multiple photo detectors 
positioned out of the incident beam plane. The configuration of the detectors is illustrated in Figure 3.4. Modern commercial PDA systems now integrate multiple photo detectors in a common housing to facilitate optical geometry calculations. This housing is referred to as the "receiving optics". The receiving optics are positioned at a particular scattering angle $\theta$, which is selected based on the relative refraction index of the particle to medium and the scattering properties of the particles measured. This will be covered in detailed in Chapter 5 . The three detectors in Figure 3.4 form angles $\psi$ with respect to the scattering plane. The particle diameter statistics come from the differences in optical path length of scattered light to each detector. All detectors identify the same Doppler Burst $f_{D}$, however, the phases of the bursts vary with the inclination angle $\psi$ of the individual detectors and with the particle diameter [5]. This concept is illustrated in Figure 3.5 where the detector signals $A$ and $B$ are out of phase based on the detector position and particle size.

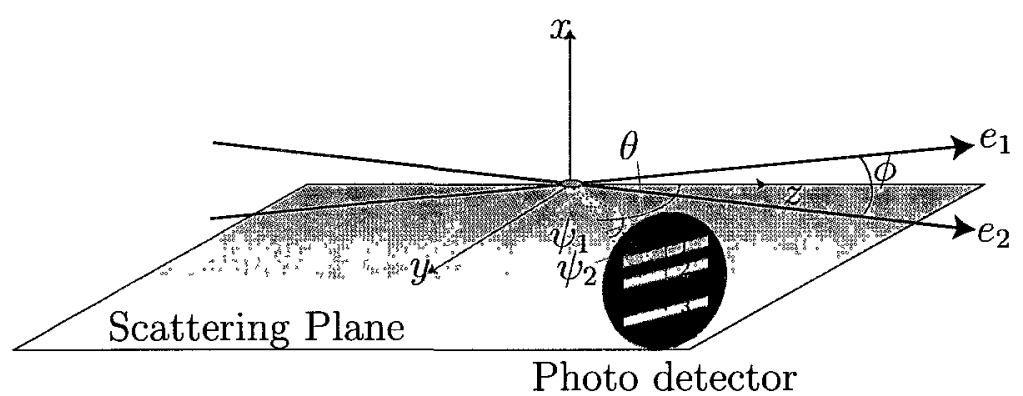

Figure 3.4: PDA configuration

If the geometrical position of the photo detectors remains unchanged, which is the case if they are embedded in a common housing, the phase difference will only be a function of the particle size. Mathematically, the phase at detector $i$ can be represented by:

$$
\Phi_{i}=\frac{\pi}{\lambda_{b}} D \beta_{i}
$$

Where $D$ is the particle diameter and $\beta_{i}$ is a detector geometrical factor which 

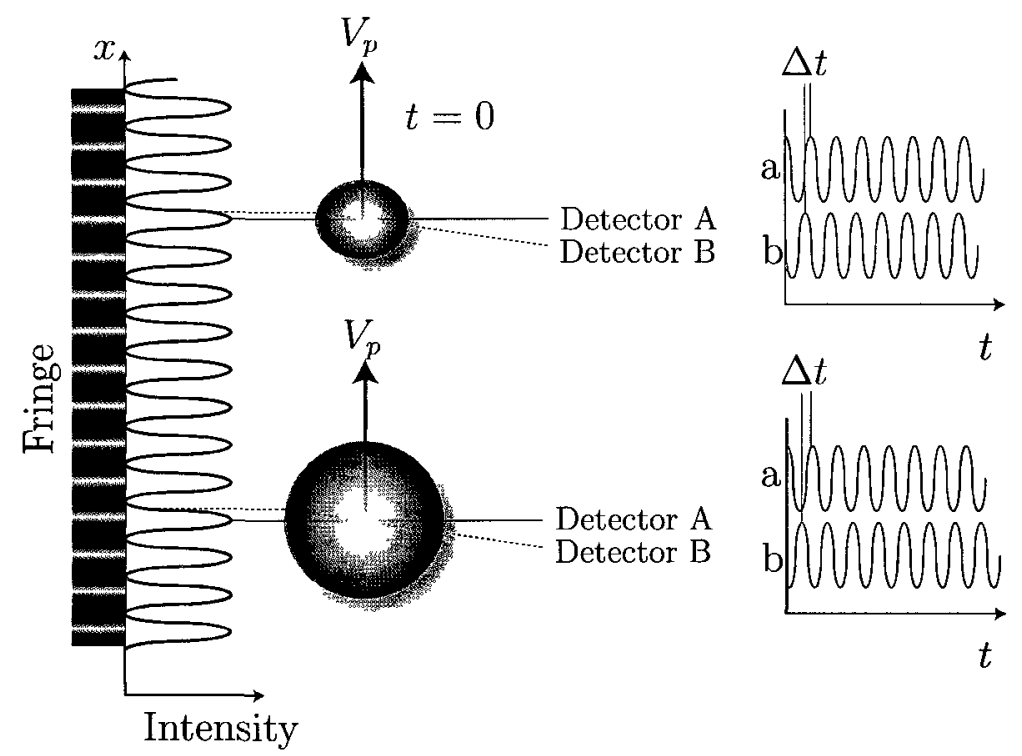

Figure 3.5: Detector signal phase difference. Adapted from [5]

is function of its spacial position, scattering mode and relative refraction index $n_{r e l}$. The particular $\beta_{i}$ from Eq. (3.9) corresponds to first order refraction, which is the scattering mode used in this study. Similar equations are available for reflection and other orders of refraction. Scattering properties of droplets are covered in detail in Chapter 5 .

$$
\beta_{i}=2\left[\sqrt{1+n_{r e l}^{2}-\sqrt{2} \cdot n_{r e l} \cdot \sqrt{f_{i+}}}-\sqrt{1+n_{r e l}^{2}-\sqrt{2} \cdot n_{r e l} \cdot \sqrt{f_{i-}}}\right]
$$

For this study, the relative refraction index is 1.46 as Viscor calibration fluid $\left(n_{2}=1.46\right)$ is injected into atmospheric air $\left(n_{1} \simeq 1\right)$. The $i^{\text {th }}$ detector geometrical factor $f_{i \pm}$ is a function of the scattering angle $\theta$, the incident beam intersection angle $\phi$ and the detector to scattering plane azimuth angle $\psi_{i}$ as:

$$
f_{i \pm}=1+\cos \frac{\phi}{2} \cdot \cos \theta \cdot \cos \psi_{i} \pm \sin \frac{\phi}{2} \cdot \sin \psi_{i}
$$

Now the phase difference of detector 1 and 2 can be represented as: 


$$
\Phi_{12}=\Phi_{2}-\Phi_{1}=\frac{\pi}{\lambda_{b}} D \cdot\left(\beta_{2}-\beta_{1}\right)
$$

Eq. (3.8) demonstrates how the droplet diameter is linearly proportional to the phase difference of photo detector 1 and 2. The sensitivity of the diameter to phase relationship can be changed by modifying the geometrical factor $\beta$. This modification will alter the slope of the relationship between diameter and phase and is extremely useful to optimize the measurement. Given that all detectors are embedded in a common housing, the easiest way to alter one of the parameters is changing the mask at the front of the receiving optics. Figure 3.6 demonstrates how the azimuth angle $\psi$ changes with different masks. As the azimuth angle decreases, $\frac{\delta \Phi}{\delta D}$ also decreases leading to a broader bandwith of particle size. However, the uncertainty also increases as a small phase error leads to a larger diameter error [5].
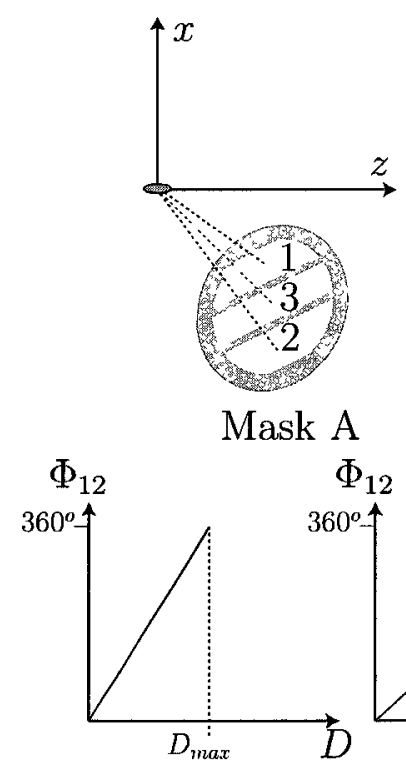

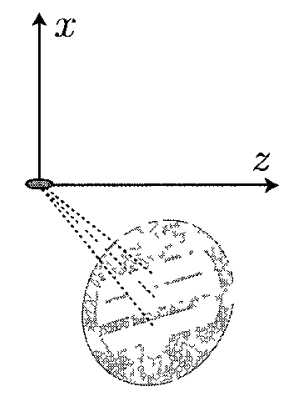

Mask B

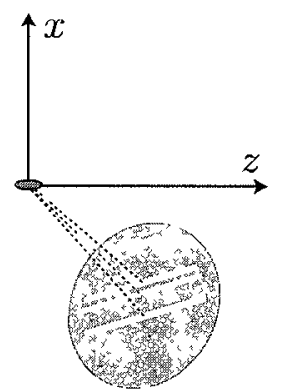

Mask C

$\Phi_{12}$

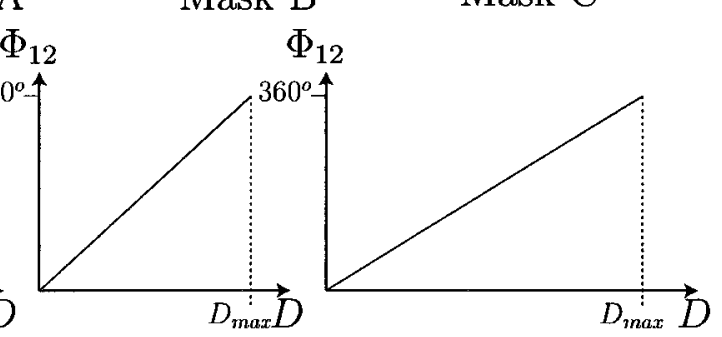

Figure 3.6: Receiving Optics mask

A problem that occurs when using only two detectors is that if the measured phase difference $\Phi_{12}$ is larger than $2 \pi$ or $360^{\circ}$, there is no way to predict the particle size. This problem is known as the " $2 \pi$ ambiguity" and is illustrated in Figure 3.7, where 
the smaller particle $V_{p 1}$ generates the same phase difference $\Phi_{12}$ as the larger particle $V_{p 2}$.
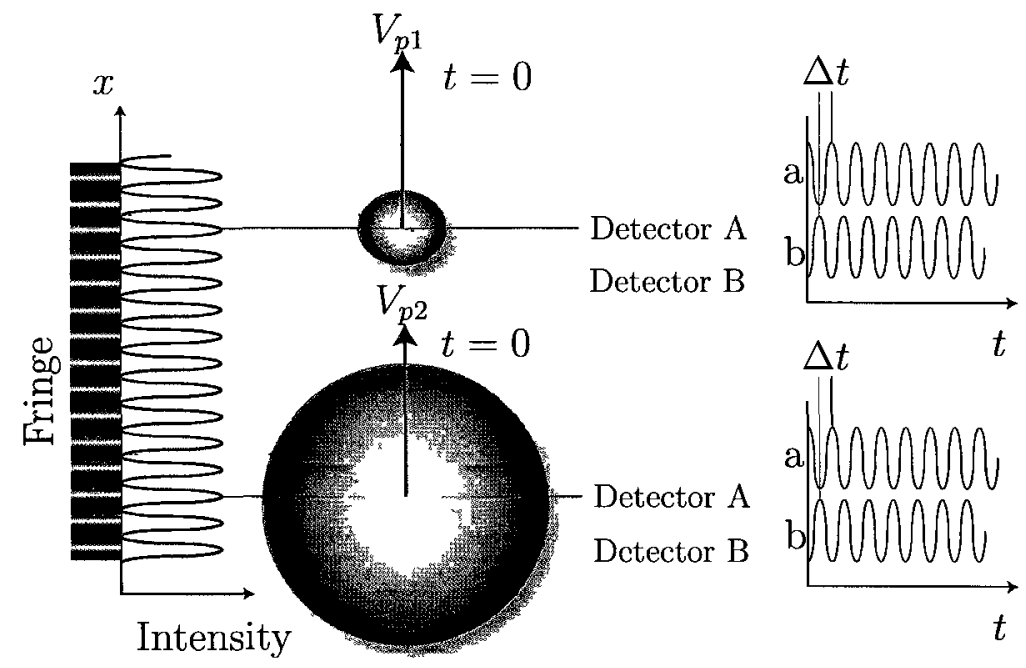

Figure 3.7: $2 \pi$ ambiguity. Adapted from [5]

Their respective $\Phi_{12}$ phase difference are exactly out of phase by one period. Determining the droplet diameter becomes impossible when the experimenter is uncertain of the size range of the measured droplets. As illustrated in Figure 3.8, the droplet diameter could be $D_{1}, D_{2}$ or even $D_{3}$. The addition of a third detector, which has a significantly different geometrical factor $\beta$, enables precise determination of the droplet diameter by comparing the location of both phase differences $\Phi_{12}$ and $\Phi_{13}$ on the diameter axis. The phase difference $\Phi_{13}$ of Figure 3.8 validates only $D_{1}$ as being the correct droplet diameter. 


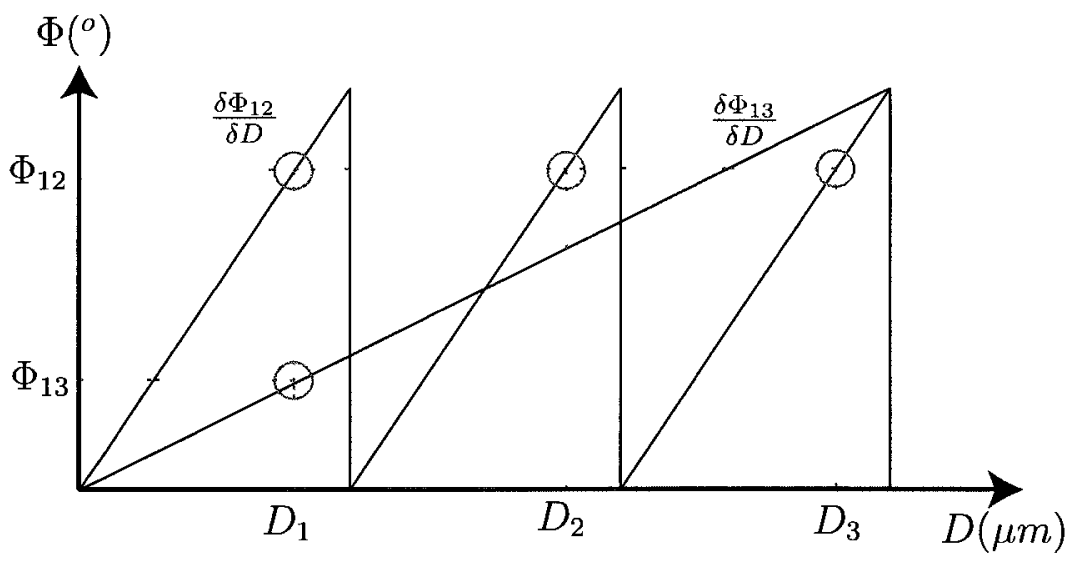

Figure 3.8: Solution to the " $2 \pi$ ambiguity" problem using 3 detectors 


\section{Chapter 4}

\section{Experimental Apparatus}

In this study, the injection spray of the IQT $\mathrm{T}^{\mathrm{TM}}$ is investigated to determine the diameter and velocity characteristics of atomized fuel droplets. The mass injected is varied from $0.98 \mathrm{~g}$ per 10 injections (IQT ${ }^{\mathrm{TM}}$ standard mass) to $0.82 \mathrm{~g}$ per 10 injections by adjusting the shim stack of the injection pump block. the injection pressure is also varied by adjusting the accumulator pressure from 175 psi to 270 psi. The measurements are performed in ambient air at atmospheric pressure. The measurements are performed at $35 \mathrm{~mm}, 55 \mathrm{~mm}, 70 \mathrm{~mm}$ and $85 \mathrm{~mm}$ downstream of the nozzle tip with 7 to 10 radial measurement points per axial position concentrated in the spray cone as illustrated in Figure 4.1.

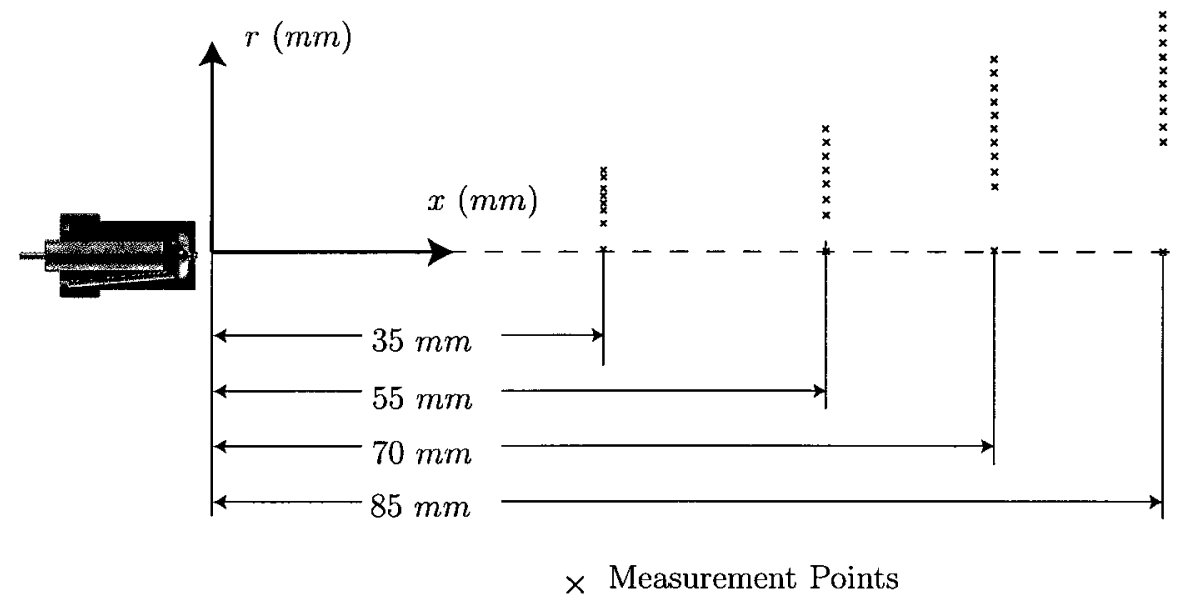

Figure 4.1: Measurement point grid 
The injection system used in this study is kept identical to the IQT ${ }^{\mathrm{TM}}$ to minimize any discrepancies due to differences between the experimental setup and the IQT $^{\mathrm{TM}}$ instrument. The spray is characterized using a Phase Doppler Anemometry system (Dantec Dynamics, Ramsey NJ). In addition, a CCD Nanosense MKII high speed camera is used to validate results of the PDA system using front light imaging. The injection event is synchronized to the CCD camera and the PDA system using a custom trigger. This enables time resolved spray characterization to be performed.

\subsection{Injection Apparatus}

The IQT ${ }^{\mathrm{TM}}$ 's injection system (Figure 4.2) is kept in the original equipment configuration as much as possible during the assembly of this experimental apparatus. First, the injection pump is installed on a custom spine plate and fitted onto a stable anti-vibration optics table. All pneumatic hoses, fuel lines and bleed lines are kept in same length as the IQT ${ }^{\mathrm{TM}}$ and installed onto the injection pump. The chamber bracket is installed to the spine plate and the chamber end cap is fastened to the chamber bracket. This chamber end cap will serve as a nozzle holder during the experiment.

The only difference between the experimental apparatus and the IQT ${ }^{\mathrm{TM}}$ is the fact that there is no heat added to the injection pump block. Normally, the IQT ${ }^{\mathrm{TM}}$ maintain the injection pump temperature to $35^{\circ} \mathrm{C}$. Without these injection pump heaters (T2 in Figure 2.2), the IQT ${ }^{\mathrm{TM}}$ 's fuel temperature increases from room temperature to approximately $35^{\circ} \mathrm{C}$ throughout the cycle of one IQT ${ }^{\mathrm{TM}}$ test. Atomization characteristics are a strong function of the fuel viscosity and surface tension which are both related to fuel temperature [16]. However, the temperature control of the pump block is not as important for these series of experiments as temperature is kept constant at 
$20^{\circ} \mathrm{C}$. The resulting droplet size statistics might be slightly biased towards a higher value as a result.

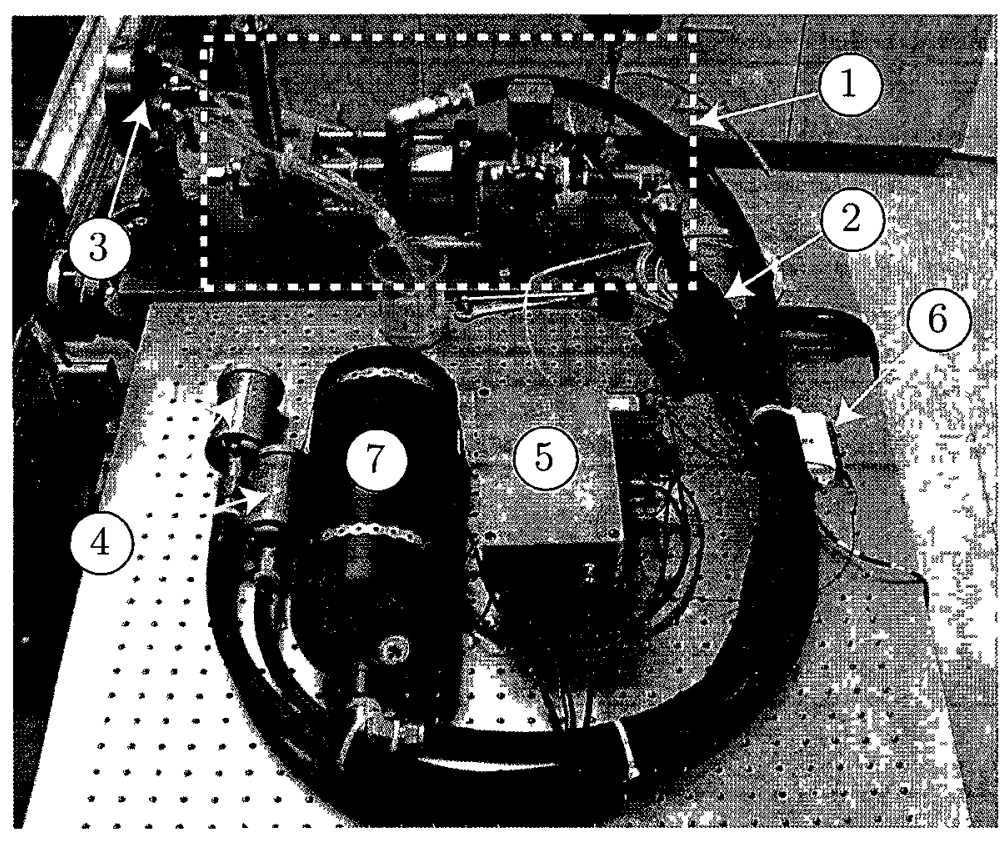

Figure 4.2: $\mathrm{IQT}^{\mathrm{TM}}$ injection apparatus

$$
\text { Where: } \begin{aligned}
1 & =\text { Injection pump } \\
2 & =\text { Solid state relay board } \\
3 & =\text { Injector, nozzle holder and needle lift sensor } \\
4 & =\text { Actuator muffler } \\
5 & =\text { Power supply and integrated trigger circuit } \\
6 & =\text { Data acquisition card } \\
7 & =\text { Air accumulator }
\end{aligned}
$$




\subsubsection{Injection Pump}

The IQT ${ }^{\mathrm{TM}}$ is fitted with a patent protected pneumatic/hydraulic fuel injection pump. The pneumatics consists of a charged accumulator, an AC solenoid valve and a pneumatic actuator. The hydraulic system consists of a barrel \& plunger and a delay pintle style injector. In the event of an injection, the charge air of the accumulator (\#13) is released using a digital negative logic signal to the solid state relay board. The relay completes the solenoid valve circuit and opens the valve (\#12). High pressure air produces a force on the pneumatic actuator(\#4), which is mechanically connected to the plunger (\#1). The actuator forces the plunger into the barrel causing a rapid increase in fuel pressure. Pressure in the fuel line increases to the point where the nozzle opening pressure (NOP) is reached. The nozzle needle lifts and discharges fuel to equilibrate pressure from the needle spring and the fuel in the injector sac. A detail representation of the injection pump is illustrated in Figure 4.3.

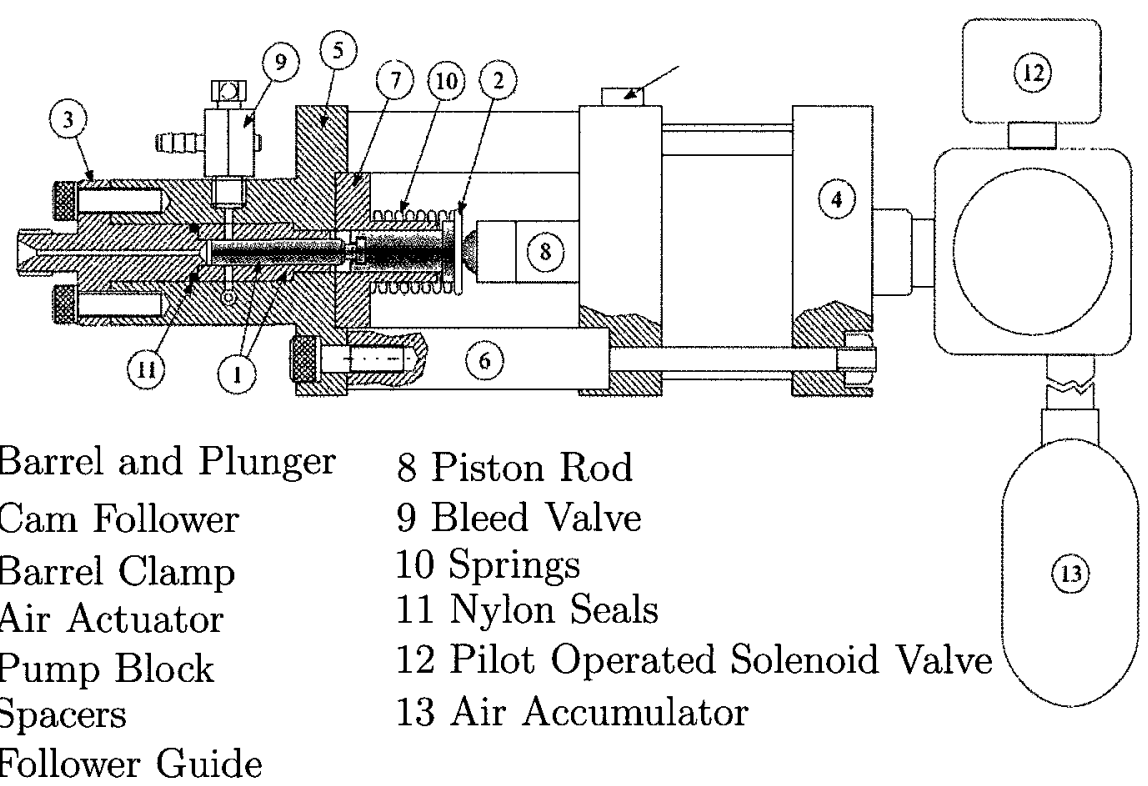

Figure 4.3: $\mathrm{IQT}^{\mathrm{TM}}$ injection pump 


\subsubsection{Injection Nozzle}

The IQT ${ }^{\mathrm{TM}}$ uses an S-type throttling pintle nozzle (Figure 4.4). The pintle nozzle is used mostly in indirect injection diesel engines. The indirect injection pre-combustion chamber is usually located above the main combustion chamber. This pre-chamber has a large amount of swirl and turbulence and a pintle nozzle is favoured for rapid mixing of fuel and air. The delay pintle was later introduced to reduce idling noise and favoured overall quieter operation [9]. The delay pintle has a reduced initial mass delivery rate compared to the original pintle nozzle. The pintle injection nozzle was selected by AET for its satisfactory hollow spray cone atomizing characteristics with a broad range of different diesel fuels with varying fuel properties. The fuel enters the nozzle from holes located in the barrel and sits in the sac surrounding the inclined surface of the needle valve. When fuel pressure surpasses the pressure caused by the the spring force acting on the needle, the needle lifts from its seat and discharges high pressure fuel. As the needle valve lifts, the initial area between the needle valve and barrel is small and therefore the injection rate is low at the start of injection. As the needle valve continues to lift the area increases reaching a maximum injection rate before the fully opened position. As the needle approaches the fully opened position, the area decreases and so does the rate of fuel injected from the nozzle [17].
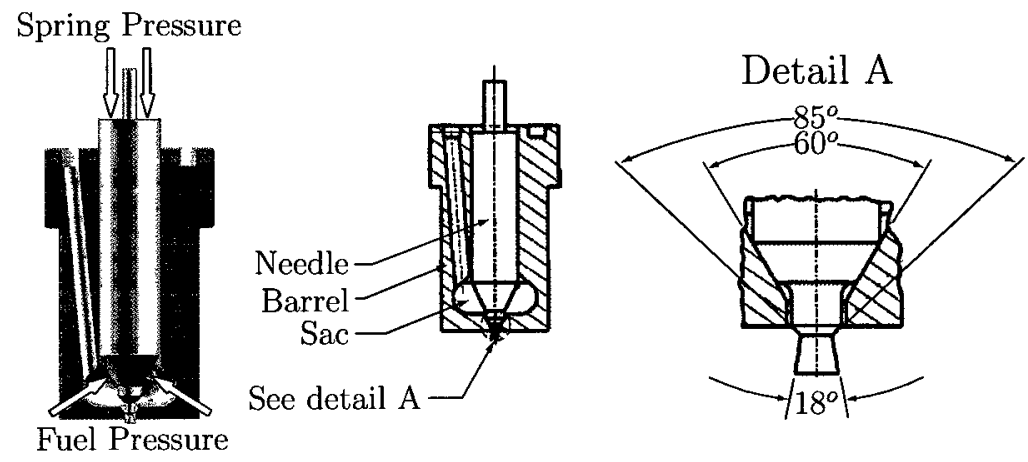

Figure 4.4: $\mathrm{IQT}^{\mathrm{TM}}$ injection nozzle 


\section{$4.2 \quad$ Electronics}

\subsubsection{Digital Signal and Data Acquisition}

The digital signal sent to the solid state relay board (Figure 4.2 component 2) is generated using a National Instruments NIDAQ 6009 USB data acquisition unit. This unit has the capability of writing digital signals on 10 channels and reading analogue inputs on 4 channels $(4 \mathrm{kHz} / \mathrm{ch})$. This data acquisition system was also used to acquire the injector nozzle needle lift trace during an injection event. The frequency of this data acquisition unit is $40 \mathrm{kHz}$ multiplexed which is appropriate to capture an adequate resolution of the IQT ${ }^{\mathrm{TM}}$ 's injector needle lift trace.

The injection sequence is programmed into the DAQ instrument using Visual Basic 6.0. The program inputs require the number of injections for the experiment and a folder to save the text file containing the comma separated values of the IQT ${ }^{\mathrm{TM}}$ needle lift trace. This needle lift trace is used to correlate atomization characteristics to the nozzle needle position, which will be covered in Chapter 6 .

\subsubsection{Needle Lift Sensor}

A Bently Nevada 3300 XL $8 \mathrm{~mm}$ proximity transducer system is used to determine the position of the injection nozzle needle. The system provides an output voltage that is directly proportional to the distance between the probe tip and the nozzle needle. The signal is sent to the NIDAQ instrument for the acquisition of the injection trace, and to the custom Schmitt Trigger for synchronizing the injection event to the PDA system and the CCD camera. 


\subsubsection{Custom Schmitt Trigger}

In order to concatenate data taken from multiple injections, a Schmidt trigger was designed and fabricated to send a TTL high pulse to the PDA system and the CCD high speed camera. The purpose of this trigger is to synchronize the PDA system and the CCD camera to the injection event. This allows multiple injections to be averaged and is necessary to characterize the spray in a time resolved manner. The trigger works as an evaluator, when the IQT ${ }^{\mathrm{TM}}$ injection pump activates, the signal from the needle lift sensor generates a voltage going from $-7 V$ (needle closed) to $-4 V$ (needle fully opened) during the injection process. The evaluator is configured such that at $-6.8 \mathrm{~V}$, the instant where the needle opens, the trigger output changes from TTL low to high. This TTL pulse is sent via a BNC cable to the PDA system and the CCD camera. This trigger allows multiple injection events to be superimposed and can later be time averaged during post-processing.

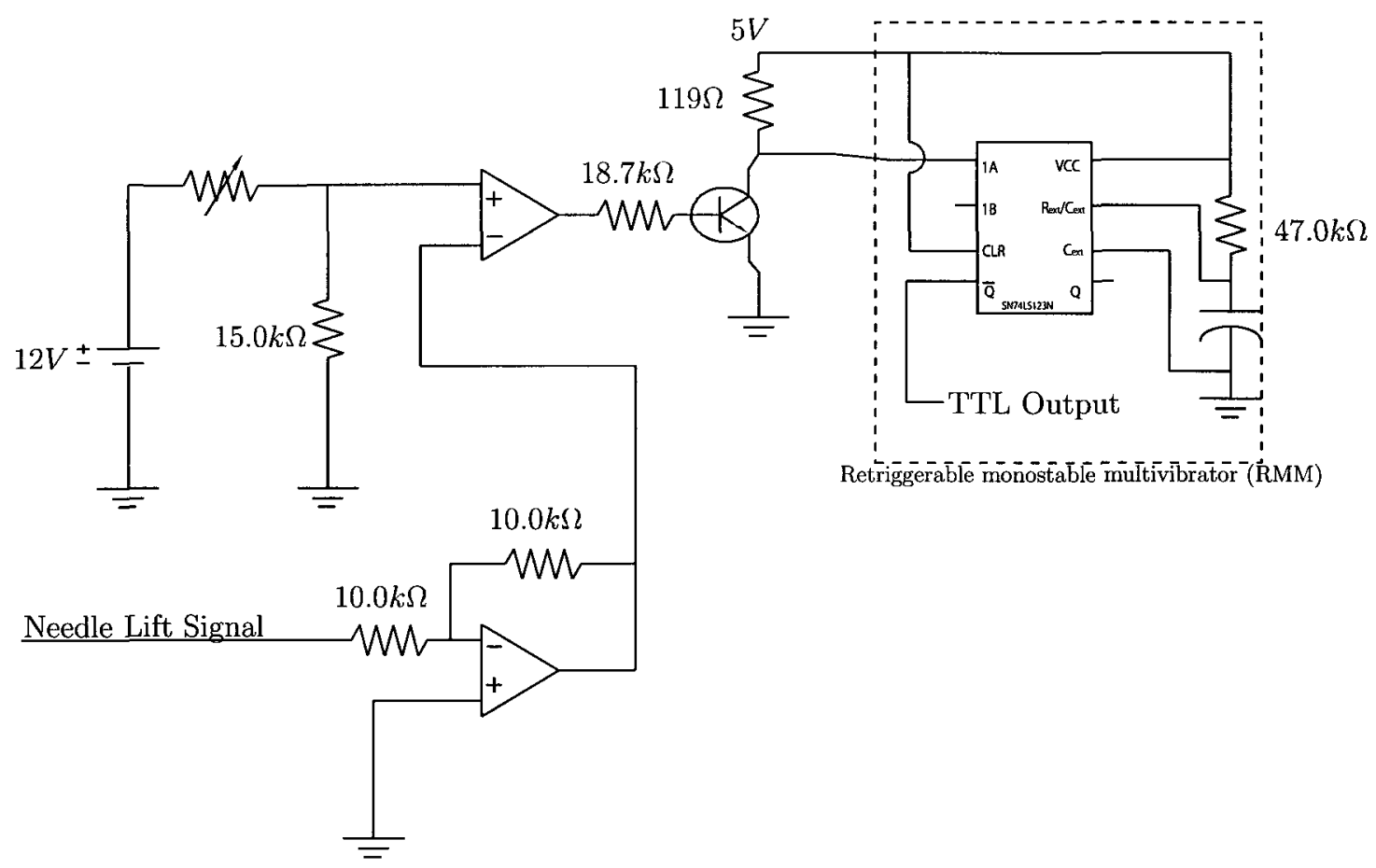

Figure 4.5: Custom Schmitt trigger and monostable vibrator circuit 
The trigger circuit is composed of two 741 operational amplifiers, one transistor and 6 resistors as depicted in Figure 4.5. The needle lift sensor static output is a negative signal in the $-7 V$ range. Therefore, the purpose of the first amplifier is to invert the signal without any gain. The inverted signal is then transmitted to the second amplifier where it compares the signal to a threshold voltage of $6.8 \mathrm{~V}$ set by the adjustable resistor. Immediately after the needle lift sensor voltage surpasses the $6.8 \mathrm{~V}$ threshold, the output of the second amplifier saturates the transistor switching the output from +5 to $0 \mathrm{~V}$.

Figure 4.6 demonstrates how secondary injections could trigger an unwarranted injection event. In order to prevent this from happening, a monostable multi-vibrator SN74LS123 chip set is used. This chip will keep a rising or falling edge signal high or low for an extended period of time. From Figure 4.5 , the $C_{\text {ext }}$ and $R_{\text {ext }}$ values were selected to provide a pulse width of $200 \mathrm{~ms}$ which is longer than the injection period of the IQT ${ }^{\mathrm{TM}}$. This will prevent secondary injections from triggering an injection event.

\subsection{Characterizing Instruments}

\subsubsection{CCD High Speed Camera}

A Nanosense MKII CCD high speed camera is used for qualitative spray characteristics such as cone angle and penetration rates. The camera features a $10 \mathrm{Khz}$ recording capability at $1280 \times 1024$ pixels with an internal memory of 2 GB. The TTL output of the trigger sends a signal to both the PDA system and the CCD camera simultaneously, therefore, the exact time of the particle flight from the nozzle tip to the measurement volume of the PDA system is known. The CCD camera indicates 


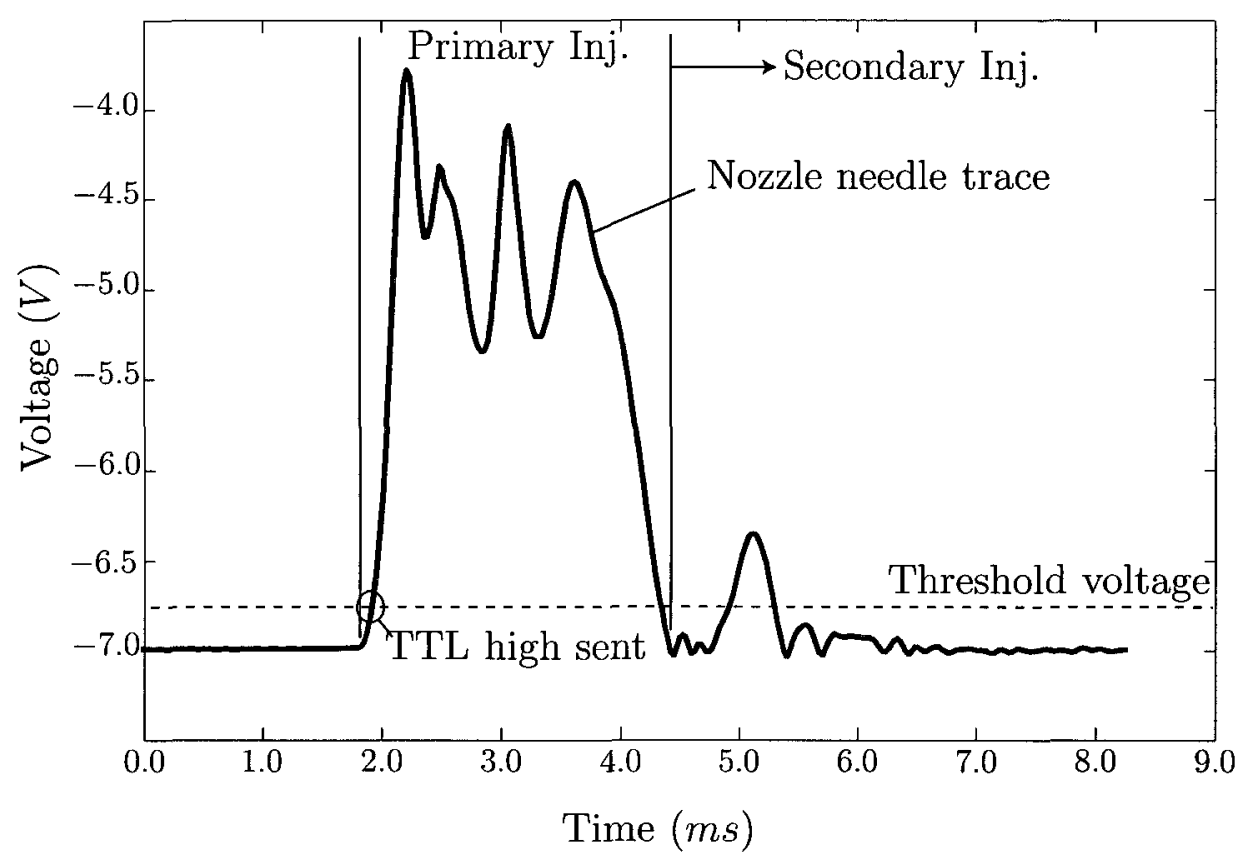

Figure 4.6: Nozzle needle trace and trigger threshold voltage

the starting time of secondary injections which will be implemented in the post processing analysis. The camera will also help validate the spray angle found using the PDA system.

\subsubsection{Phase Doppler Anemometry}

Phase Doppler Anemometry (PDA) is a non-intrusive point measurement technique used to characterize the size and velocity of spherical particles. This technique is mostly used in characterizing aerosols or other types of jets. PDA is an extension of Laser Doppler Velocimetry (LDV) where the main difference is the fact that PDA is limited to the presence of a single particle in the measurement technique. The PDA system is coupled with a 2D traversing support system which moves the transmitter and receiver allowing multiple measurement points to be interrogated without realigning the optics. The PDA system used in this study is provided by Dantec Dynamics. Transmitting optics are composed of a Spectra-Physics Stabilite 2017 laser 
(refer to Figure 4.7), which produces a multiline beam with a maximum power output of roughly $7 \mathrm{~W}$. The beam then enters the transmission box where a quartz prism splits the incoming beam into two $514.5 \mathrm{~nm}$ and two $488.0 \mathrm{~nm}$ lines. Both lines are connected to a FiberFlow $60 \mathrm{~mm}$ 2D transmitter using fiber optics. The receiving optics utilize a Hidense FiberPDA 57x50 receiver. A BSA P60 Flow and Particle Processor is coupled to the FiberPDA Box. The FiberPDA box is connected to the receiving optics using optical fiber. The FiberPDA box contains three photomultiplier tubes which convert the optical Doppler burst into current. The current is then send to the BSA P60 processor where the frequency content of the signal is analyzed by Fast Fourier Transforms (FFT). The processor sends result files to the post-processing software on a desktop computer. A detailed description of this measurement method is covered in Chapter 3 and Chapter 5. 


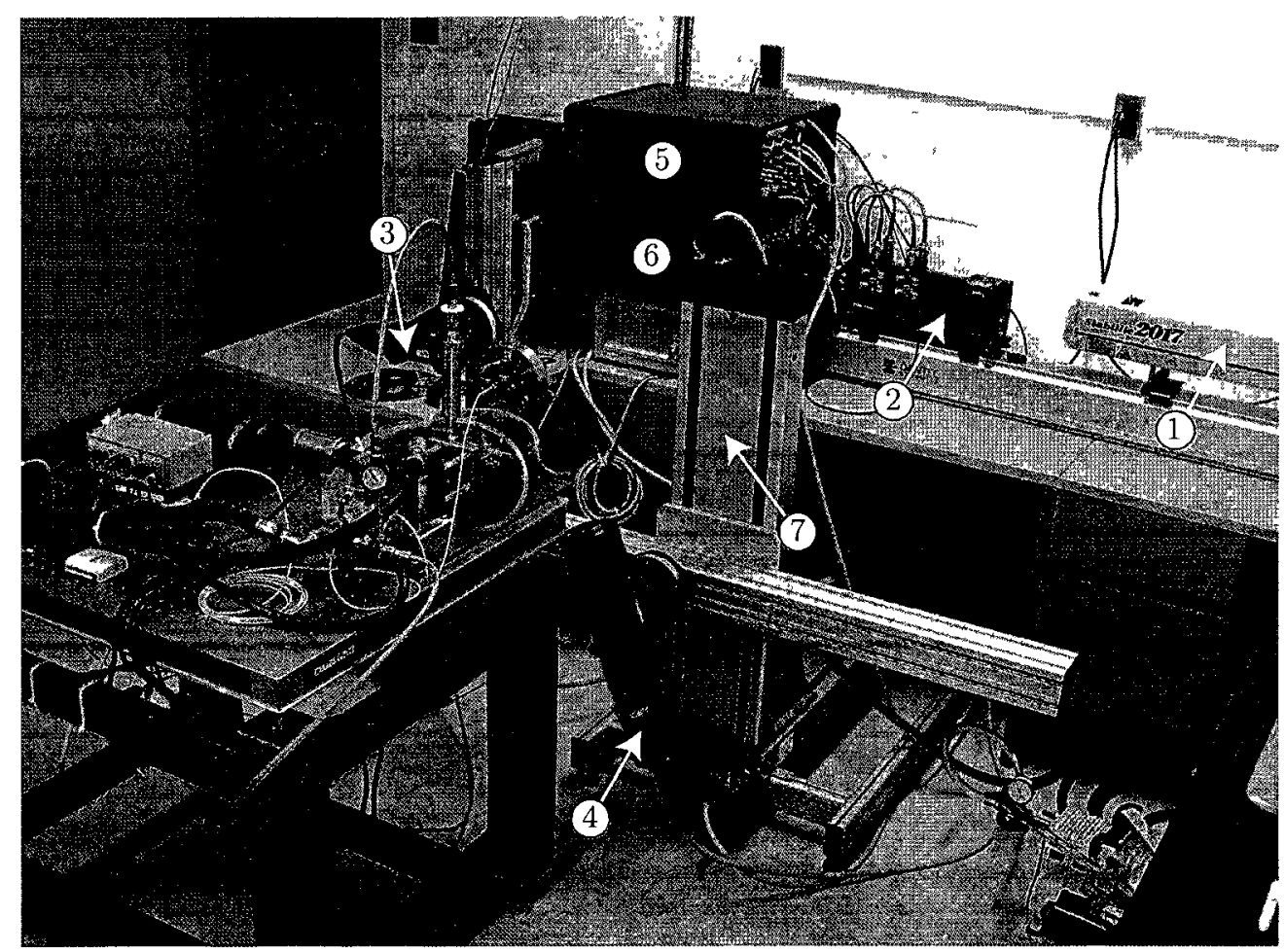

Figure 4.7: PDA system and experimental apparatus

$$
\text { Where: } \begin{aligned}
1 & =\text { Spectra-Physics Stabilite } 2017 \text { LASER } \\
2 & =\text { Beam Splitter } \\
3 & =60 \mathrm{~mm} 2 \mathrm{D} \text { Transmitting Probe } \\
4 & =\text { HiDense FiberPDA Receiver } \\
5 & =\text { Fiber PDA Detector } \\
6 & =\text { BSA P60 Processor } \\
7 & =2 \mathrm{D} \text { Traverse System }
\end{aligned}
$$

\subsection{Summary of Process Diagram}

The experimental process diagram is illustrated in Figure 4.8. The injection command is sent from the DAQ card to the solid state relay. The solid state relay opens the air accumulator valve and the injection pump is activated. The nozzle discharges fuel 
while the needle lift sensor monitors the needle position. The needle position signal is sent to the trigger and the DAQ card simultaneously. The DAQ card records the needle trace while the trigger sends a TTL rising edge signal to the BSA processor and the CCD camera simultaneously to signal the start of injection. The FiberPDA detects the optical Doppler burst of fuel droplets as they cross the measurement volume and sends a current proportional to the burst intensity to the BSA processor. The BSA processor analyzes the frequency content of the burst and calculates the droplet velocity and diameter as a function of the time following the trigger signal. The results are then sent to the BSA Flow Software.

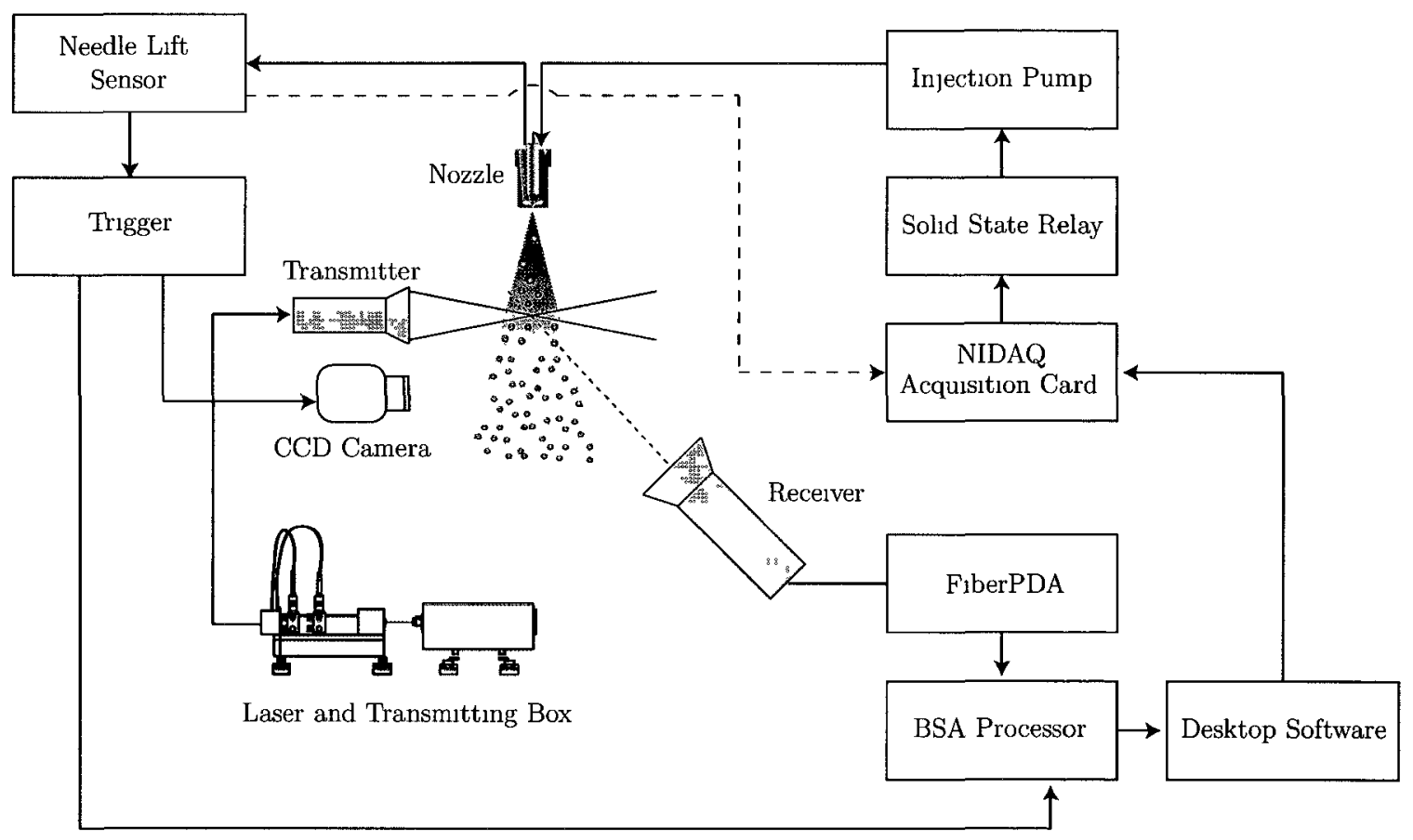

Figure 4.8: Experimental process diagram 


\section{Chapter 5}

\section{Measurement Parameter Selection}

Phase Doppler measurement techniques originate from the properties of the scattered light from a droplet or particle in the measurement volume. The scattered light is imaged on photo detectors at a strategic position in space as discussed in Chapter 3. The scattered light transports information of the droplet velocity and size characteristics. It is important to understand the scattering properties of droplets in their surrounding medium in order to be able to make proper decisions in the selection of PDA measurement parameters.

\subsection{Droplet Scattering Properties}

There are a few light scattering theories used in phase Doppler measurements depending of the size of the droplets. The important parameter in deciding what type of scattering analysis really depends on the relative size of the droplet diameter to the incident light wavelength. This nondimensional parameter is known as the Mie parameter, where $d_{p}$ is the particle diameter and $\lambda_{b}$ is the incident light wavelength:

$$
x_{m}=\frac{\pi d_{p}}{\lambda_{b}}
$$


For droplets having a Mie parameter in the range of $1<x_{m}<10$, the wavelength of light is comparable to the droplet size. Scattering intensity maps of these droplets must be calculated using Lorenz-Mie theory. This theory is based on changing the incident planar wave into a sum of spherical waves over the particle. The wave equation is then solved and a solution for the spherical waves is obtained by superposition [45]. This technique is only used for very small particles where the particle size is much smaller than the incident beam diameter. For particles having similar size or larger than the incident beam waist, this technique must be modified given that the incident wave is no longer homogeneous. This leads to the Fourier-Lorenz Mie Theory (FLMT) where the inhomogeneous wave is replaced by a series of homogeneous waves using Fourier transforms and solved using the wave equation.

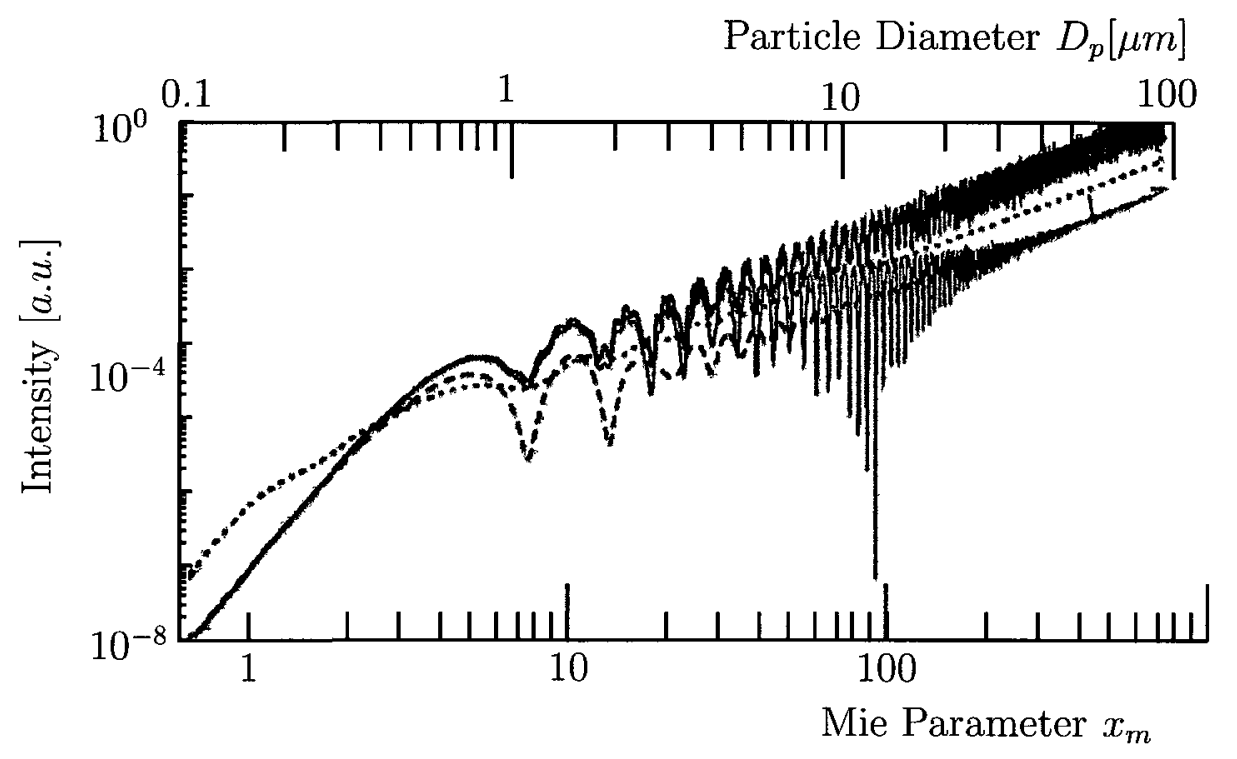

Figure 5.1: Scattered light intensity as a function of Mie parameter $x_{m}$ and particle size for water $n_{r e l}=1.33$. Solid line represents $\theta=30^{\circ}$ and parallel polarization $[6]$

Figure 5.1 illustrates the intensity of scattered light, in arbitrary units, at $\theta=30^{\circ}$ as a function of particle diameter and Mie parameter $x_{m}$. For small particles having a Mie number of 10 or below, the intensity of scattered light increases with the 
$6^{\text {th }}$ power of the particle diameter. This scattering behaviour is known as Rayleigh scattering. This will represent particles of size smaller than $1.64 \mu \mathrm{m}$ for the phase Doppler configuration in this study. Scattered intensity of particles having a Mie number of 10 and above increases with the square of the particle diameter [6].

Another technique for analyzing the scattering intensity field and mostly used for representation of phase Doppler measurements is the geometrical optics (GO) approach. GO is only valid for particles significantly larger than the wavelength of the incident light. As its name implies, it solves the scattered field by ray tracing through the particle. GO is also visualized assuming homogeneous plane waves however, extended geometric optics (EGO) can be used for visualizing inhomogeneous plane waves (i.e. large particle size compared to incident beam diameter). EGO is solved on a small portion of the particle where GO is applicable and a series of incident beams are traced towards the detector. This is how most logarithmic intensity plots as a function of scattering angle are constructed. Figure 5.2 demonstrates the importance of scatterin $g$ intensity as a function of the scattering angle $\theta$. The selection of the receiving optics spatial position with respect to the measurement volume is based on the scattering information of these plots.
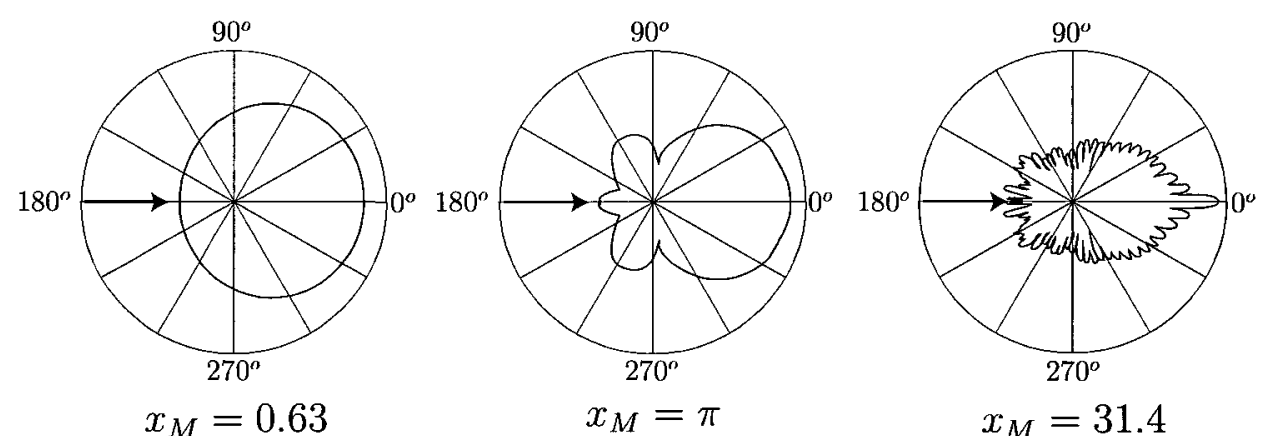

$x_{M}=\pi$

$x_{M}=31.4$

Figure 5.2: Scattering intensity for three different Mie parameter values computed for water droplets in air using EGO [6] 


\subsubsection{Scattering Modes}

There are many modes and orders of scattering which all depend on the physical properties of the particle and surrounding medium. The two main modes of scattering are reflection and $1^{\text {st }}$ order refraction. Understanding the scattering intensity of these two modes is paramount for optimization of the PDA measurement technique. The most important parameter is the relative refraction index of the particle compared to the medium $n_{\text {rel }}$.

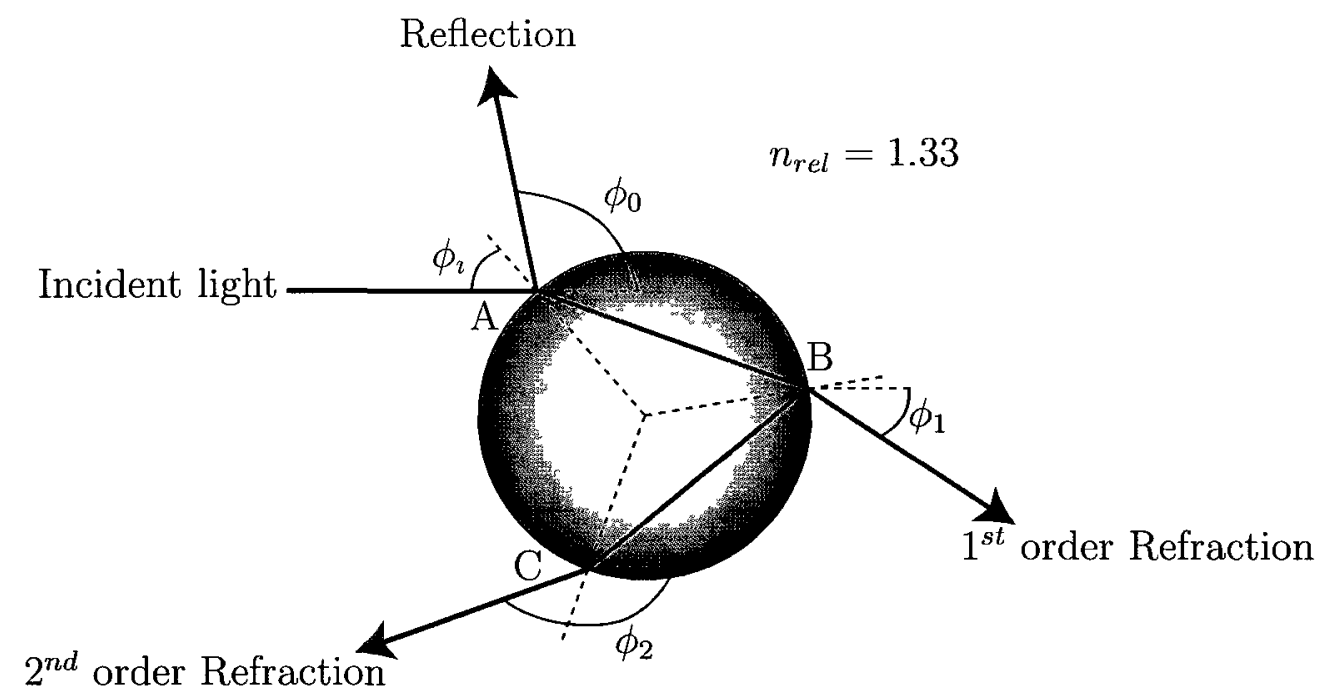

Figure 5.3: Scattered light from water droplet where $d_{p} \gg \lambda_{b}, n_{r e l}=1.33$ [5]

Figure 5.3 demonstrates the multiple modes of light scattering from a water droplet with $n_{r e l}=1.33$ and $d_{p} \gg \lambda_{b}$. When the incident beam interacts with the particle at point $\mathrm{A}$, a portion of the light is reflected at angle $\phi_{0}$. The other portion of the incident beam that penetrated the droplet escapes at point B with angle $\phi_{1}$. This is known as $1^{\text {st }}$ order refraction. Internal reflection is also present at point $\mathrm{B}$ which causes the other portion of the beam to escape at point $\mathrm{C}$ with angle $\phi_{2}$. This is defined as $2^{\text {nd }}$ order refraction. All these scattering angles can be found using GO knowing the incident angle $\phi_{\iota}$ and the relative refraction index of the particle to medium $n_{r e l}$ as: 


$$
\begin{gathered}
\phi_{0}=\pi-2 \phi_{i} \\
\phi_{1}=2\left[\arcsin \left(\frac{\sin \phi_{i}}{n_{r e l}}\right)-\phi_{i}\right] \\
\phi_{2}=4 \arcsin \left(\frac{\sin \phi_{i}}{n_{r e l}}\right)-2 \phi_{i}-\pi
\end{gathered}
$$

Figure 5.4 illustrates the intensity of scattered light computed by EGO with respect to the different modes and orders of scattering from a finite incident beam [6]. The relevant information from this figure is the dominant mode of scattering as a function of the scattering angle $\theta$. For example, if the selected scattering angle is $\theta=30^{\circ}$ then, the scattered light will be dominated by first order refraction. However, there still exists reflection at this scattering angle. Although the intensity is lower, a larger droplet in the measurement volume could scatter similar light intensity by reflection then a smaller droplet by refraction given that scattering intensity is also function of the droplet size. If these two droplets were to be in the measurement volume at the same instance, the particle would be rejected during validation stage of the PDA BSA processor.

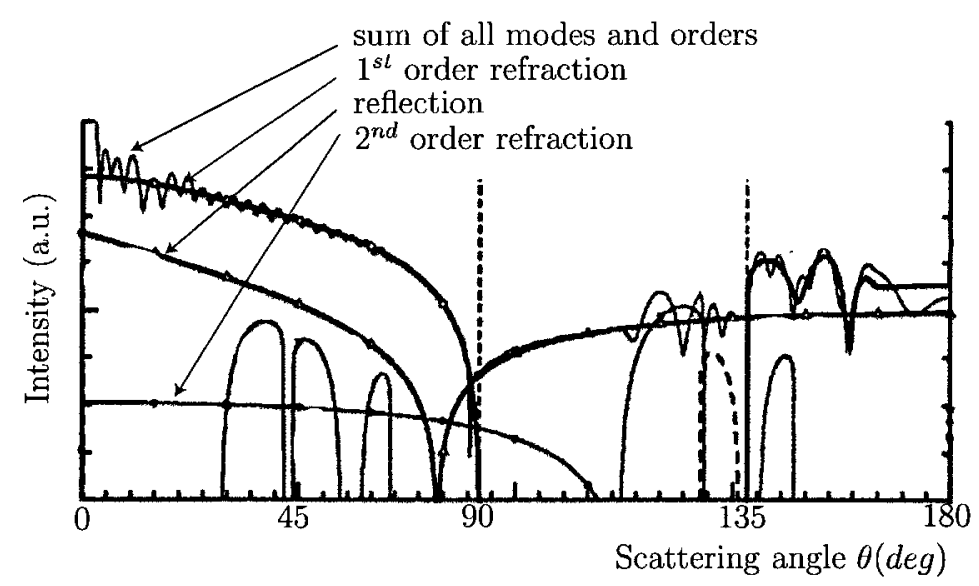

Figure 5.4: Intensity distribution of different scattering modes for $d_{p}=10 \mu \mathrm{m}$ water droplet calculated using extended geometrical optics EGO. [6] 


\subsubsection{Scattering Angle Selection}

Another important factor of scattering intensity is the incident light polarization. Scattering properties of a diesel droplet in atmospheric ambient air as a function of scattering angle and incident light polarization is represented in Figure 5.5. Generally, first order refraction (forward scattering) dominates anywhere between $30^{\circ}$ and $70^{\circ}$ using parallel polarization while reflection is only dominant using perpendicular polarization between $89^{\circ}$ and $148^{\circ}$. If for some reason, forward scattering accessibility is impossible (e.g. looking into a combustion chamber with only one optical port), second order refraction (back scattering) dominates anywhere between $148^{\circ}$ and $212^{\circ}$ with either incident light polarization. The important scattering angle to note is $\theta_{b 0}$, where using parallel polarized light, reflection is completely absent. This angle is known as the Brewster angle [5]. Using this scattering angle will reduce the occurrence of a multiple Doppler burst caused by the Gaussian intensity profile which will be discussed in Section 5.2.3. The scattering angle chosen for this study is $68.8^{\circ}$ which is the Brewster angle of Viscor calibration fluid in ambient atmospheric air $n_{\text {rel }}=1.46[6]$.
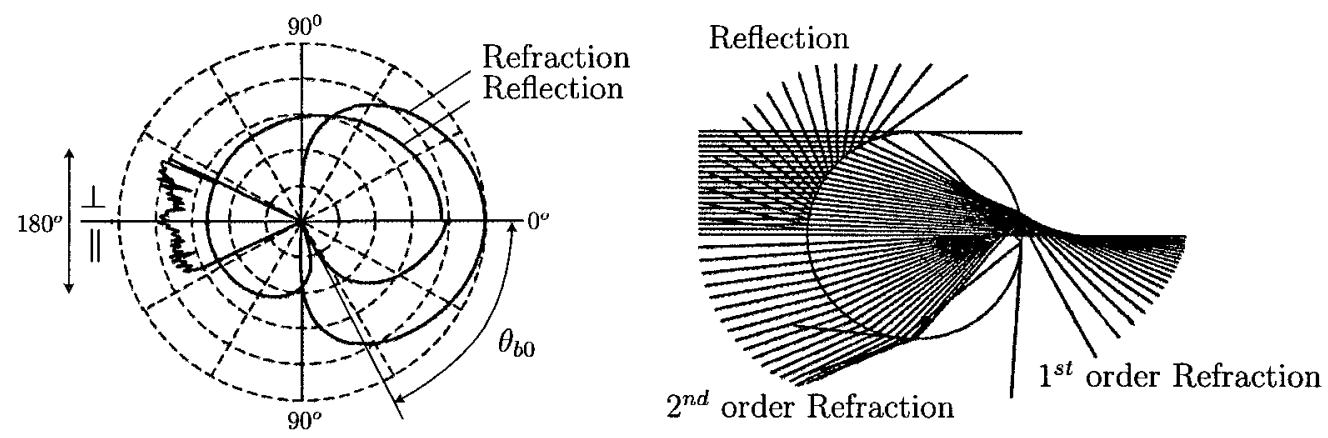

Figure 5.5: Droplet of diesel oil in atmospheric air $n_{\text {rel }}=1.46$. Left: Logarithmic light intensity. Right: GO representation [5] 


\subsection{Selection of Instrument Parameters}

\subsubsection{Laser Power}

Laser power is an important parameter to optimize to ensure proper measurement. The difficulty of characterizing a dense liquid spray is attributed to the beam attenuation through the dense droplet cloud surrounding the measurement volume. Laser power must be set such that the Doppler burst caused by droplets surpasses the burst threshold. Laser power is not the limiting factor in this case as a $7 \mathrm{~W}$ continuous wave (CW) laser source is available. Recalling from Section 5.1, the scattering intensity is a strong function of the droplet size, therefore, if the laser power is set such that the scattered intensity of small droplets is insufficient to surpass the burst threshold, the smaller droplets will not be detected. At lower power levels this would lead to biased high droplet size statistics [8].

The effect of laser power on droplet diameter is investigated at an axial distance of $35 \mathrm{~mm}$ from the nozzle tip where the droplet density is highest. Figure 5.6(a) illustrates the dependency of the average droplet diameter on laser power. The laser power in this test is varied from $100 \mathrm{~mW}$ to $5 \mathrm{~W}$. The AMD along with its estimated error (see Section 5.4) are computed after 30 injections for each power level. The results suggest an exponential relationship between laser power and the AMD which is in agreement with findings from Araneo et al. [46] and Kapulla et al. [47]. As expected, lower power levels lead to biased high AMD statistics.

Similar velocity measurements were found to be not as dependent on laser power as compared to the AMD. As long as the burst threshold is met, velocity histograms and time resolved velocities will be similar for different laser power levels. This result is demonstrated in Figure 5.6(b) where the average axial velocity remains relatively 


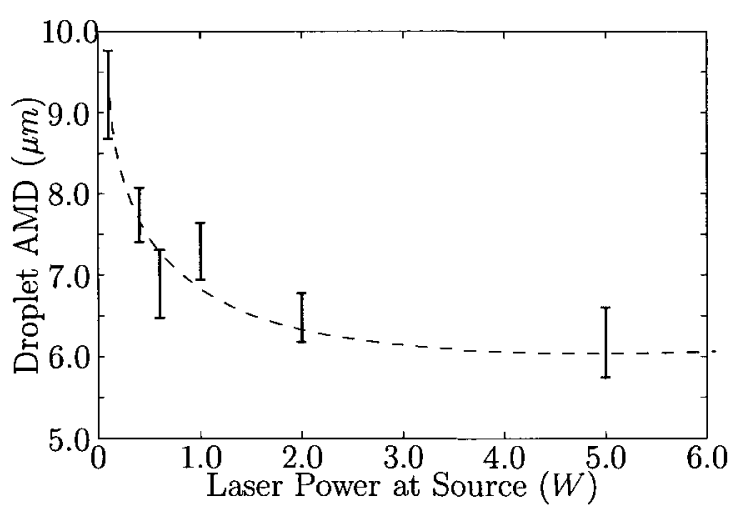

(a) Droplet diameter dependency on incident laser power

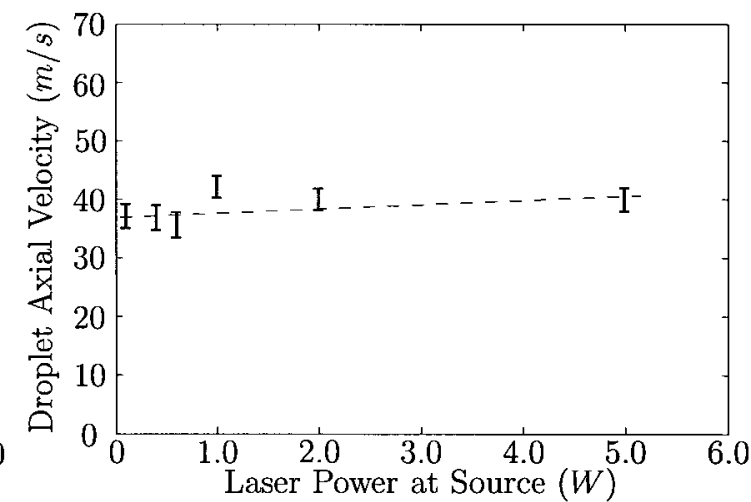

(b) Droplet velocity dependency on incident laser power

Figure 5.6: Effect of laser power on the droplet AMD and velocity

constant for all laser power levels. The laser power selected for this characterization study is $2 W$ given that Figure 5.6(a) suggests at least $1 W$ of laser power for proper AMD statistics. Also, using a laser power of $2 W$ will ensure proper penetration of the incident light to the measurement volume in high droplet proximity regions.

\subsubsection{High Voltage, and SNR}

The photo detector high voltage (HV) is varied from $500 \mathrm{~V}$ to $1400 \mathrm{~V}$ on each of the detectors to identify the best HV for this study. The effect of HV on the acquisition of data is similar to laser power, however, an increase in voltage generates noisier signals. Therefore, $\mathrm{HV}$ is usually set with the signal to noise ratio (SNR) at the same instance. The data rate was the determining factor as an increase in data rate usually represents better acquisition, unless too much noise is misinterpreted as droplets [8]. Experiments were conducted at $85 \mathrm{~mm}$ away from the nozzle axis. Another criteria for the selection of $\mathrm{HV}$ is the anode current of each detector which must be similar. It was found that a $\mathrm{HV}$ of $1000 \mathrm{~V}$ with a gain of $24 \mathrm{~dB}$ on the LDA1 channel and $1200 \mathrm{~V}, 24 d B$ on LDA4 channel resulted in best data rate and overall time resolved velocity. 


\subsubsection{Receiver Slit Width}

The PDA system available features an aperture slit in front of the three photo detectors as illustrated in Figure 5.7. PDA is restricted to a single particle in the measurement volume for validation of the Doppler burst. A slit is added to the receiving optics to reduce this possibility in highly dense flows by truncating the measurement volume in the z-direction. Another reason for the slit is to control the area of investigation to a known width. The size of the measurement volume is not precise enough to associate a flux to droplet velocities and sizes. The slit ensures that the area, which the particles cross the measurement volume, is well defined. This makes flux measurements possible [5].

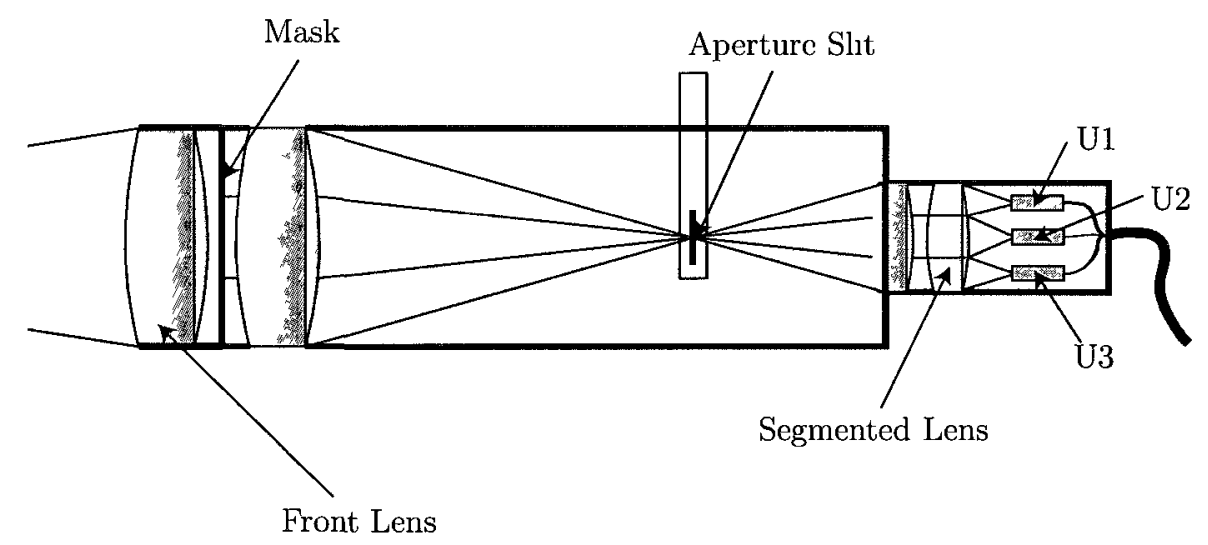

Figure 5.7: FiberFlow PDA Probe (57X80) layout. Photo detectors are represented as U1,U2 and U3.

Multiple particles could trigger the Doppler burst threshold if their sizes were relatively small compared to the measurement volume. Figure 5.8 demonstrates how the slit makes measurements possible in dense sprays where the droplet proximity is high. The measurement volume is represented by the light gray ellipsoid where multiple particles can be identified at time $=t$. The dominant scattering mode of these particles is first order refraction since the receiver is positioned at $\theta=30^{\circ}$. The slit in this case blocks first order refraction of each particle except droplet A. Reflection of 
droplet B is also identified, however, knowing the scattering properties discussed earlier and setting $\theta$ at $30^{\circ}$, the intensity of the Doppler burst of the reflected droplet is orders of magnitude weaker than the refracted light. The result will be the validation of droplet A only.

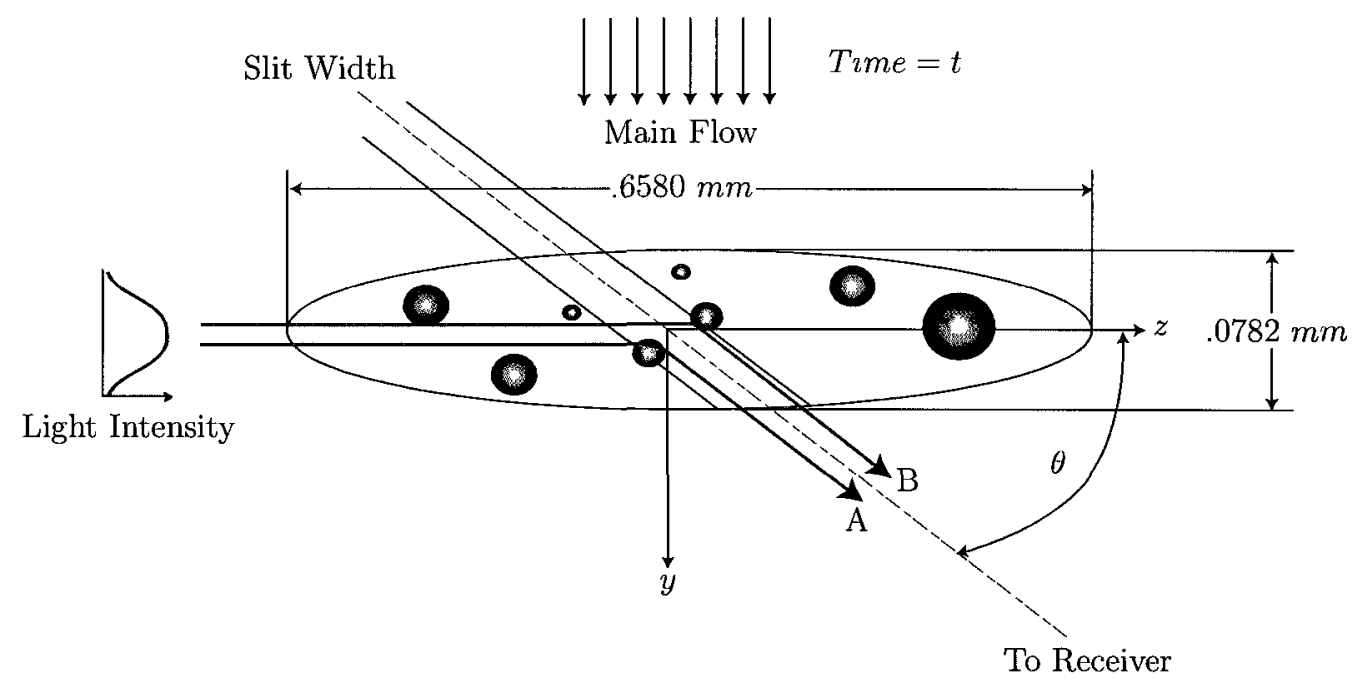

Figure 5.8: Importance of the slit aperture for Gaussian intensity distributed measurement volumes. Adapted from Wigley et al. [7]

The slit selection for dense diesel sprays should be as small as the receiver permits [8]. This will reduce the occurrence of multiple droplets in the measurement volume in the high droplet proximity regions. The disadvantage of using a small slit is that data rate will decrease since most droplets are blocked by the slit. To compensate for the reduction of data rate, the injection sample size is increased. Figure 5.9 demonstrates the axial velocity of a dense diesel spray measured by Payri et al. [8]. Using the smaller slit improves the measurement in the highly dense region $(1.0 \mathrm{~ms}<t<3.0 \mathrm{~ms})$ of the injection. 

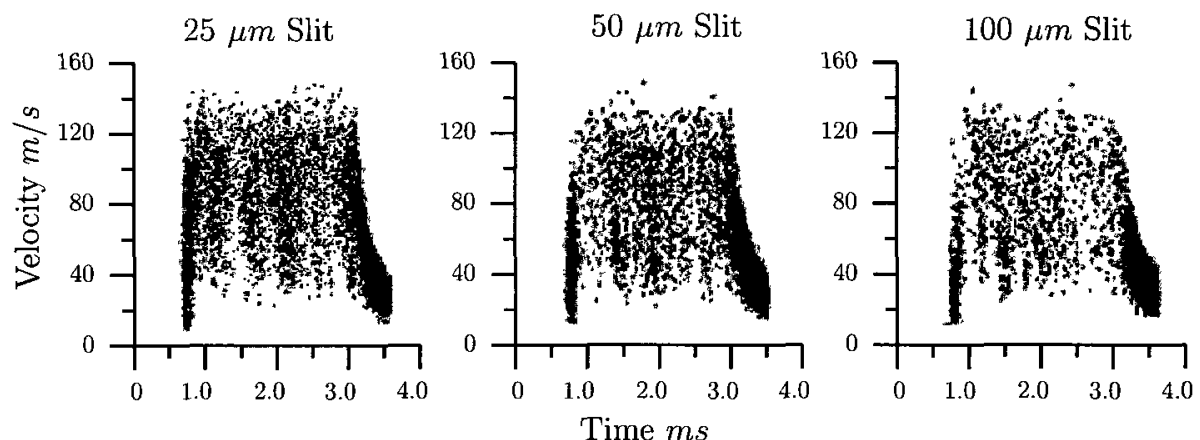

Figure 5.9: Influence of the receiving optics slit aperture: velocity results $40 \mathrm{~mm}$ downstream from the orifice, $1.4 \mathrm{~mm}$ off-axis, $P_{i n \jmath}=500 \mathrm{bar}, P_{a m b}=7 \mathrm{bar}$ (air) [8]

\subsubsection{Injection Sample Size}

Its important to determine how the average droplet velocity and size at a spatial position changes as a function of the injection sample size in order to select the proper sampling for this study. Figure 5.10(a) demonstrates how the droplet AMD converges to a value of $8.4 \mu \mathrm{m}$ then fluctuates within the $95 \%$ confidence uncertainty derived from Section 5.4.

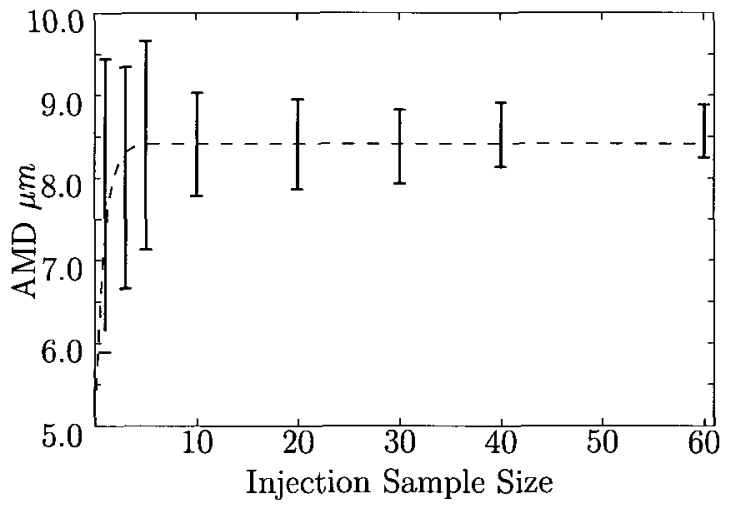

(a) AMD vs Sample Size

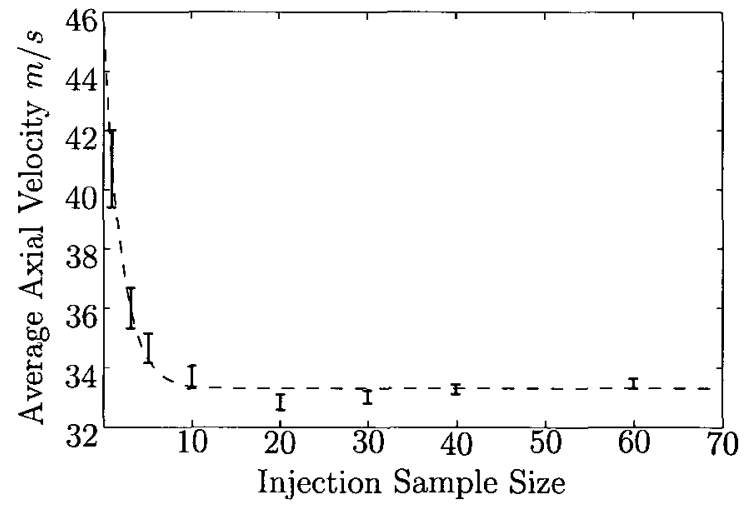

(b) Axial Velocity vs Sample Size

Figure 5.10: AMD and average axial velocity as a function of injection sample size.

Similarly, Figure 5.10(b) demonstrates similar results for the average droplet velocity. A sample value of 20 assures the convergence of the average axial velocity. Based 
on these results, an injection sampling size of 30 per spatial position is selected. This assures proper convergence and improves the sampling statistics. Sampling 30 injections will require 15 minutes to complete one spatial position.

\subsubsection{Transmitting Optics}

There are two focal lengths available for the transmitting optics. The selection of the $160 \mathrm{~mm}$ lens over the $400 \mathrm{~mm}$ lens is made from the optimization study of Payri et al. [8]. The measurement volume must be kept as small as possible for dense sprays such as diesel injection [48]. The focal length of the $160 \mathrm{~mm}$ lens produces an intersection half angle $\phi / 2=6.772^{\circ}$ while the $400 \mathrm{~mm}$ lens produces a half angle of $2.72^{\circ}$. Knowing the incident beam diameter $d_{b}=0.076 \mathrm{~mm}$, the size of the two ellipsoid volume is then approximated by Eq. (3.5). The resulting ellipsoid volume is represented in Table 5.1. The $160 \mathrm{~mm}$ produces the smallest ellipsoid volume resulting in a measurement volume which has the largest energy density.

Table 5.1: Measurement volume dimension comparison

\begin{tabular}{|l|c|c|c|}
\hline Lens & $\delta x$ & $\delta y$ & $\delta z$ \\
\hline $160 \mathrm{~mm}$ & $.0782 \mathrm{~mm}$ & $.0776 \mathrm{~mm}$ & $.658 \mathrm{~mm}$ \\
$400 \mathrm{~mm}$ & $.1941 \mathrm{~mm}$ & $.1941 \mathrm{~mm}$ & $4.091 \mathrm{~mm}$ \\
\hline
\end{tabular}

\subsubsection{Receiving Optics}

The focal length selection on the receiving optics controls the azimuth angle $\psi_{i}$ between the three photo detectors within the receiving optics. As discussed in Section 3 , the azimuth angle controls the size range of measured droplets. From previous work completed by Koo and Martin [49], diesel droplets sizes should range between $0 \mu m<d_{p}<200 \mu m$ for a standard valve covered orifice injector. However, drop 
size statistics measured using PDA are not available for a throttling pintle style injector used within the IQT ${ }^{\mathrm{TM}}$. Ryan et al. [16] estimated SMD values of $20 \mu \mathrm{m}$ from a pintle style injector using high speed photography and a Malvern laser-diffraction drop sizing instrument. Therefore, a $310 \mathrm{~mm}$ focal length receiving lens is selected to provide a size range of $0 \mu m<d_{p}<156.5 \mu m$.

Another, adjustment that enables a more precise control of the azimuth angle is the aperture mask positioned between the receiving lens and the segmented lens within the receiver. For the optical configuration selected above, the size range can further be controlled by the mask. The following values are summarized in Table 5.2.

Table 5.2: Maximum droplet size vs mask selection

\begin{tabular}{|c|c|c|}
\hline Mask A & Mask B & Mask C \\
\hline $0-40.43 \mu \mathrm{m}$ & $0-65.66 \mu \mathrm{m}$ & $0-156.5 \mu \mathrm{m}$ \\
\hline
\end{tabular}

All masks are evaluated by sampling 30 injections. Mask A and B discard many droplets as the cutoff sizes are $40.43 \mu \mathrm{m}$ and $65.66 \mu \mathrm{m}$, respectively. This does not have a significant effect on the average axial velocity and AMD. However, SMD changes significantly given that larger droplets have a larger contribution to the SMD. Figure 5.11(b) demonstrates the droplet diameter as a function of time after the SOI. Although the count of large droplets is small, they would have been rejected using Mask A and B resulting in erroneous SMD values. This demonstrates the importance of investigating the time resolved droplet size 5.11(b) to select the proper Mask as opposed to the histogram of Figure 5.11(a) which would lead one to believe that no droplets above $40 \mu \mathrm{m}$ exist in the spray. 


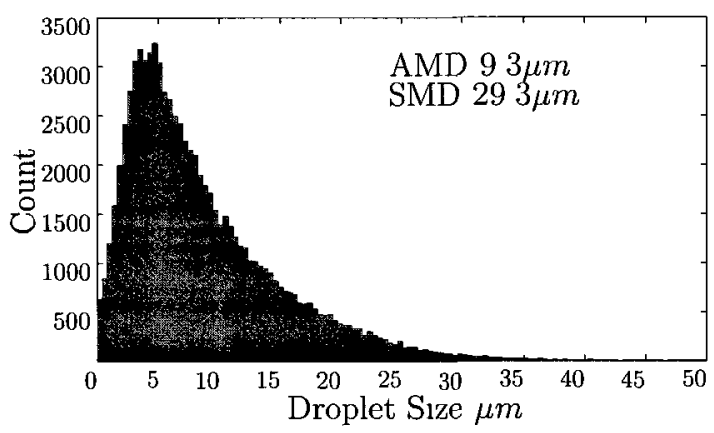

(a) Diameter Histogram

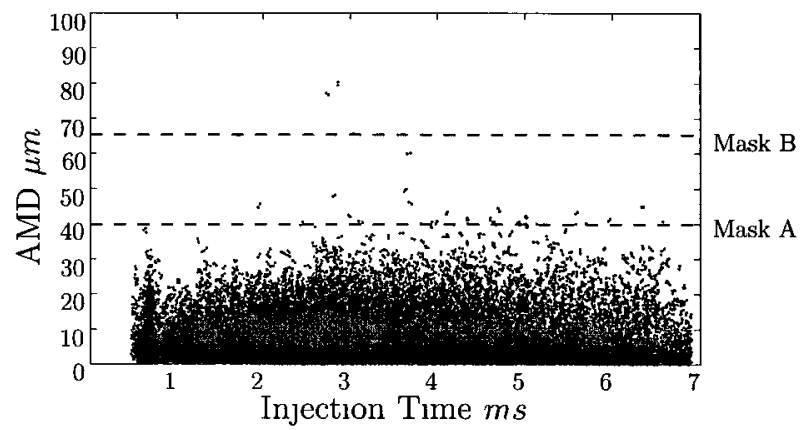

(b) Diameter vs Injection time

Figure 5.11: Diameter hıstogram and time resolved droplet size (30 injections Mask C)

\subsubsection{Processor Limitations}

The selection of the $160 \mathrm{~mm}$ focal length lens limits the maximum measurable velocity bandwidth to $62 \mathrm{~m} / \mathrm{s}$. A processor upgrade to the PDA system would allow a larger measurement bandwidth of $250 \mathrm{~m} / \mathrm{s}$, however this option is more expensive and the lead time would be too long for the time requirements of this study. A solution to this limitation is to complete bandwidth measurements by modifying the digital filtering frequency. This frequency can be adjusted such that the central velocity is set as $31 \mathrm{~m} / \mathrm{s}$ measuring velocities from $0 \mathrm{~m} / \mathrm{s}$ to $62 \mathrm{~m} / \mathrm{s}$ (Figure 5.12 (a)). Now to capture the faster moving droplets, the experiment is redone while adjusting the frequency such that the central velocity is now $93 \mathrm{~m} / \mathrm{s}$ allowing measurements from $62 \mathrm{~m} / \mathrm{s}$ to $124 \mathrm{~m} / \mathrm{s}$ (Figure 5.12(b)). Then, the data can be concatenated and analyzed yielding velocity bandwidth measurements of 0 to $124 \mathrm{~m} / \mathrm{s}$. Figure $5.12(\mathrm{c})$ demonstrates how the time resolved velocity data fits after the concatenation using this method. 


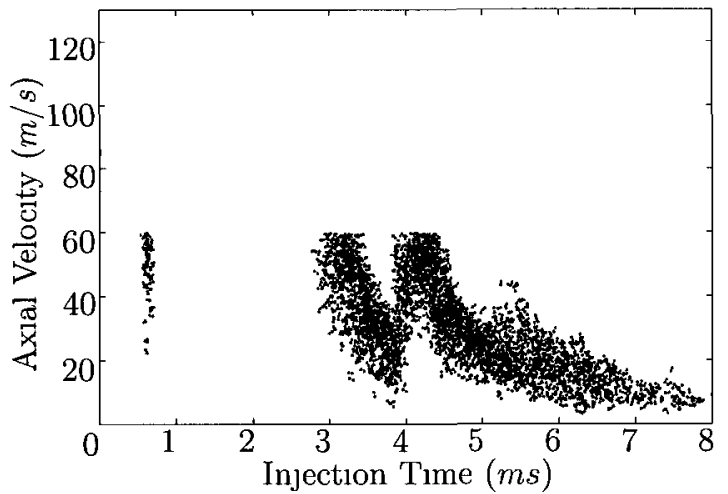

(a) Central velocity at $32 \mathrm{~m} / \mathrm{s}$

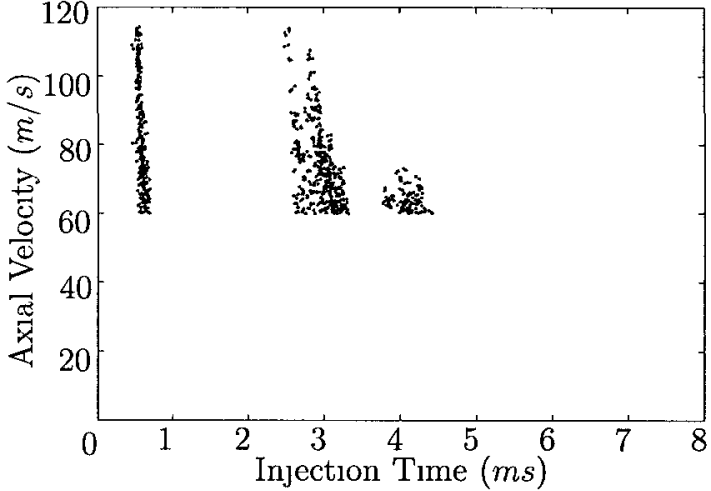

(b) Central velocity at $90 \mathrm{~m} / \mathrm{s}$

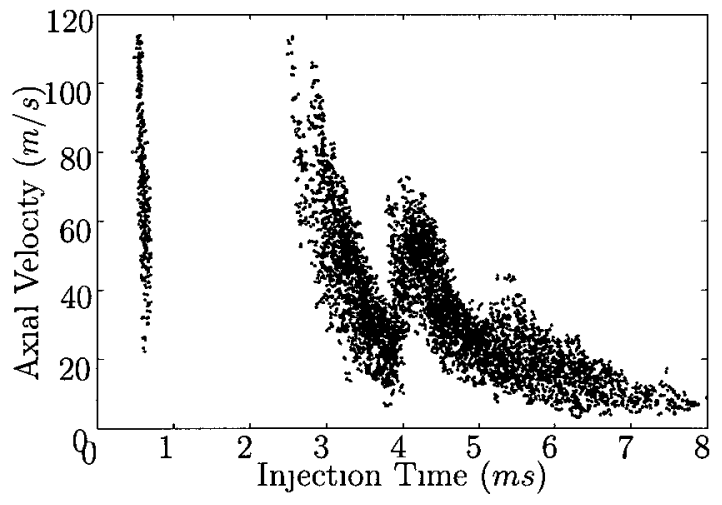

(c) Coupling data

Figure 5.12: Data coupling analysis (concatenation process) 


\subsubsection{Signal Validation Process}

The Doppler burst signal validation procedure is important to understand in order to optimize the data acquisition of the PDA measurement. One of the main reasons for poor data rate and poor droplet validation is a noisy signal. All Doppler burst signals from the photo detectors contain noise. The noise could come from the electronics as shot noise, Johnson noise, dark current and could even be caused by the scattering physics of light [6]. The idea here is not to investigate all different sources of noise relative to the signal but rather to understand how to validate a droplet event through the noise.
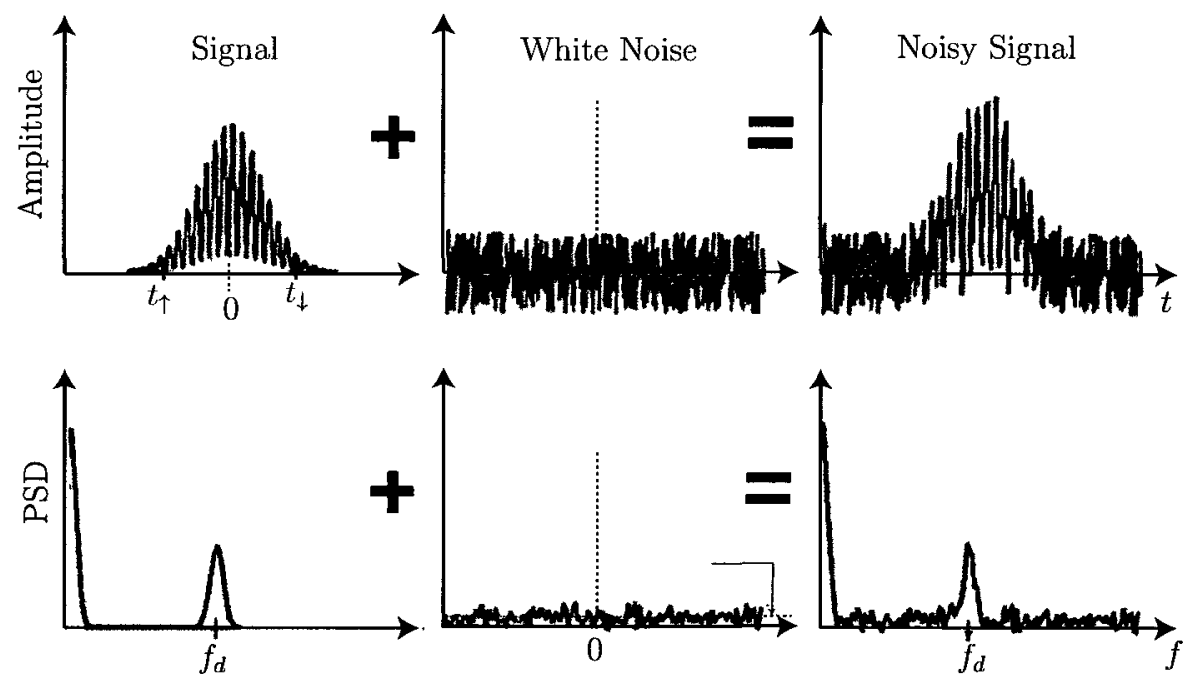

Figure 5.13: Doppler burst and noise contribution [6]

Figure 5.13 demonstrates an analytical Doppler burst signal with the addition of white noise. Most of the noise is frequency dependent and can therefore be removed using band bass filtering, however, white noise will always be present in the signal. White noise appears as a random signal with a flat power spectral density curve (PSD) occurring over the whole spectrum [50]. The second row of Figure 5.13 demonstrates the signal after the FFT is performed. This is known as the PSD of a signal. From 
this PSD, the doppler burst frequency $f_{D}$ can be identified within the noise. This particular Doppler burst is only validated if the SNR of the burst is above the SNR threshold selected.

The procedure to optimize the validation rate is to improve the SNR by balancing the photo detector signal gain and by selecting the appropriate laser power and scattering angle discussed earlier. Figure 5.14 demonstrates the SNR of a signal where $\sigma_{n}^{2} / f_{s}$ is the average noise amplitude and $\Delta f$ is the bandwidth selected for the measurement. The SNR of this signal is the ratio of the PSD area A versus area B as:

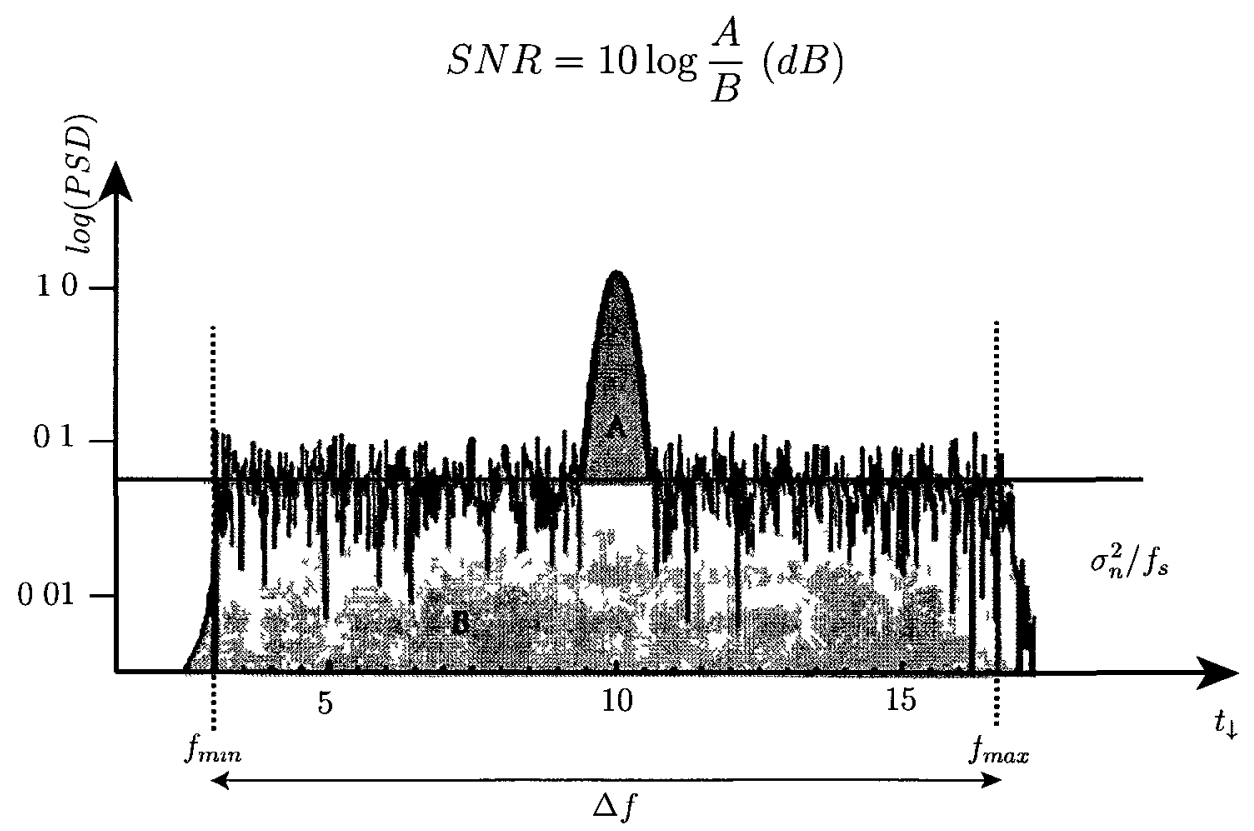

Figure 5.14: Signal to noise ratio [6]

The signal is validated only if SNR $>\mathrm{SNR}_{\text {Threshold. }}$. The $\mathrm{SNR}_{\text {Threshold }}$ set at $0 d B$ was found to generate the best data rate for this study. After the selection of the SNR, it is important to investigate for any noise validation. A good method that was found to detect noise validation is to investigate the velocity as a function of time after the SOI. If fast droplets $(40 \mathrm{~m} / \mathrm{s}$ to $130 \mathrm{~m} / \mathrm{s})$ are identified long after the 
SOI, they are likely the result of noise validation. This would suggest increasing the $\mathrm{SNR}_{\text {threshold }}$ to eliminate noise validation.

\subsection{Parameter Selection Summary}

Table 5.3 shows a summary of all instrument parameters selected for this study.

Table 5.3: Summary of instrument parameter selection

\begin{tabular}{|c|c|c|c|c|}
\hline Parameter & LDA1 & LDA4 & PDA U1 & PDA U2 \\
\hline HV & $1000 \mathrm{~V}$ & $1200 \mathrm{~V}$ & $1000 \mathrm{~V}$ & $1000 \mathrm{~V}$ \\
\hline Signal gain & $24 d B$ & $24 d B$ & & \\
\hline Signal to noise ratio & $0 d B$ & $0 d B$ & & \\
\hline Level validation & 2 & 2 & & \\
\hline Wavelength $\lambda_{b}$ & & & $514.5 \mu \mathrm{m}$ & $488.0 \mu \mathrm{m}$ \\
\hline Transmitter focal length & & & $160 \mathrm{~mm}$ & $160 \mathrm{~mm}$ \\
\hline Beam diameter & & & $1.35 \mathrm{~mm}$ & $1.35 \mathrm{~mm}$ \\
\hline Frequency shift & & & $40 \mathrm{MHz}$ & $40 \mathrm{MHz}$ \\
\hline Fringe number & & & 35 & 35 \\
\hline Fringe spacing & & & $2.182 \mu m$ & $2.069 \mu m$ \\
\hline Beam half angle $\theta / 2$ & & & $6.772^{\circ}$ & $6.772^{\circ}$ \\
\hline Probe volume $\delta x$ & & & $0.0782 \mathrm{~mm}$ & $0.0742 \mathrm{~mm}$ \\
\hline Probe volume $\delta y$ & & & $0.0776 \mathrm{~mm}$ & $0.0736 \mathrm{~mm}$ \\
\hline Probe volume $\delta z$ & & & $0.658 \mathrm{~mm}$ & $0.624 \mathrm{~mm}$ \\
\hline Scattering angle & & & $67^{\circ}$ & $67^{\circ}$ \\
\hline Receiver focal length & & & $310 \mathrm{~mm}$ & $310 \mathrm{~mm}$ \\
\hline Fringe direction & & & Negative & Negative \\
\hline Scattering mode & & & Refraction & Refraction \\
\hline Aperture mask & & & Mask C & Mask C \\
\hline Spherical validation band & & & $25 \%$ & $25 \%$ \\
\hline Max detectable diameter & & & $156.5 \mu m$ & $156.5 \mu \mathrm{m}$ \\
\hline Relative refraction index & & & 1.46 & 1.46 \\
\hline Phase factor $\Phi_{12}$ & & & 7.745 & 7.745 \\
\hline Phase factor $\Phi_{13}$ & & & 1.672 & 1.672 \\
\hline
\end{tabular}




\subsection{Uncertainty Analysis}

Phase Doppler measurements are based on physical principles which do not require calibration of the instrument prior to measurements [47]. However, as presented above, many system parameters do affect the droplet size measurement statistics and must be optimized prior to the measurement.

Phase Doppler measurement derives physical quantities from the frequency and not the intensity of the scattered light. Therefore, the incident beam attenuation through a dense particle region does not introduce error to the measurement as long as the burst threshold of crossing droplets is met [6]. Many authors investigated the effect of system parameters on the measured droplet size (Albrecht et al. 2003 [6], Kapulla et al. 2005 [47], Araneo et al. 2000 [46] ) and reported findings similar to the optimization study performed in the previous sections. However, very few attempts have been done to investigate the propagation of the error throughout the system.

\subsubsection{Velocity error}

The velocity uncertainty develops from four sources of errors. The beam intersecting angle, beam wavelength, Bragg cell frequency shift and the photo detector signal frequency uncertainty. In order to investigate the velocity error propagation, the incident beam wavelength was given an error of $1 \%$ and the intersection angle was assumed to have a $0.5 \%$ error [51]. As for the detector, Yoshioka et al. 2005 [52] successfully measured the uncertainty of photo detectors using twice-modulated light. The detector uncertainty in the frequency range of LDA measurements is roughly $0.66 \%$. Table 5.4 summarizes the different sources of error.

Figure 5.15(a) illustrates the contribution of the optical parameters to the velocity error. The derived velocity uncertainty is found using the ASME measurement 
Table 5.4: longcaption

\begin{tabular}{lllll} 
& Source & Symbol & Equation & Error \\
\hline \multirow{3}{*}{ Optics } & Beam intersection angle & $\theta$ & Eq. (3.2) & $0.5 \%[51]$ \\
& Bragg cell & $f_{o}$ & Eq. (3.7) & $<1 \%[51]$ \\
& Wavelength & $\lambda_{b}$ & Eq. (3.2) & $<1 \%$ \\
\hline Electronics & Photo detrector & $f_{D}$ & Eq. (3.7) & $0.66 \%[52]$
\end{tabular}

uncertainty reference [53], Eq. (3.6) and (3.7). The resulting conservative velocity uncertainty is demonstrated in Figure 5.15(b).

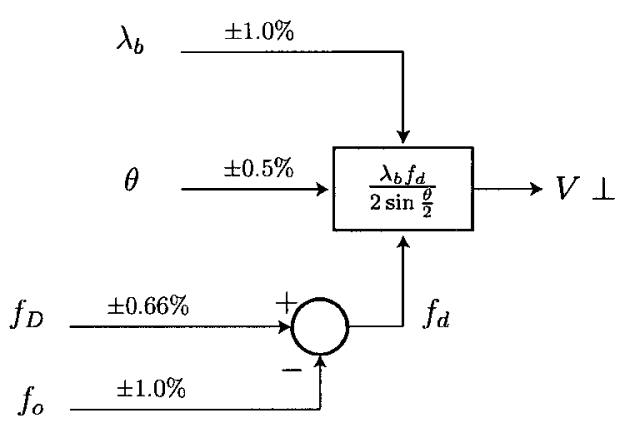

(a) Error propagation in velocity measurement

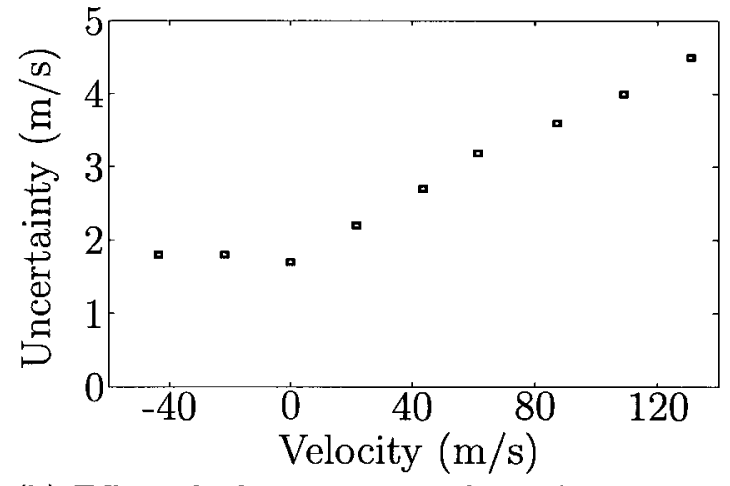

(b) Effect of velocity magnitude on the measurement uncertainty

Figure 5.15: Uncertainty analysis

\subsubsection{Diameter}

The droplet diameter found from phase Doppler measurement is derived in a completely different way than the velocity. The phase difference between signals from multiple detectors is of interest rather than the frequency content. Performing a similar uncertainty analysis is difficult given that the elevation angle of the detectors is unknown to the PDA user. The elevation angle is a software derived parameter 
associated with the receiver mask selected. Below is a list of parameters required for the determination of droplet size:

- Incident beam wavelength

- Incident beam intersection angle

- Scattering angle of the receiver with respect to the incident beam

- Elevation angle of each photo detectors with respect to the scattering plane

- Relative refraction index of the droplet/medium

- Phase difference of the Doppler burst between multiple detectors

A different approach to evaluate the uncertainty of the droplet size was suggested by Kapulla et al. [47]. The scatter of the diameter estimate can be treated as normally distributed [47]. Therefore, the determination of the AMD $\left(D_{10}\right)$ uncertainty can be performed by first determining if each measured droplet is statistically independent. An auto-covariance equation is introduced in order to determine the correlation between each successive droplet [54]. Once the correlation function is known, the independent lag time $\tau$ between droplets can be found. This lag time determines the frequency at which droplets are statistically independent. For example, if droplet data is acquired at a mean rate of $\simeq 1,000 \mathrm{~Hz}$ with a lag time of $2 \mathrm{~ms}$ found using the correlation function, this would suggest that only half the droplets are statistically independent.

The arithmetic mean diameter variance estimator $\sigma_{D_{10}}^{2}$ can be found using the following equations [6]:

$$
\sigma_{d}^{2}=\frac{1}{N} \sum_{i=1}^{N}\left(d_{i}-D_{10}\right)^{2}
$$




$$
\sigma_{D_{10}}^{2}=\frac{\sigma_{d}^{2}}{N}
$$

The correlation function package implemented in the BSA software was not available for this study. However, authors have suggested a lag time between $2<\tau<4 m s$ for typical atomization sprays which results in a frequency of $250<f<500 \mathrm{~Hz}[47]$. Given the average acquisition rate of this study fluctuated around $1 \mathrm{kHz}$ suggests that only a quarter of droplets acquired were statistically independent. As an example, the droplet statistic measured at $85 \mathrm{~mm}$ downstream at a radial position of $21 \mathrm{~mm}$ is:

- The total droplet count: $N=2687$

- The arithmetic mean diameter: $D_{10}=7.46 \mu \mathrm{m}$

- The variance of the diameter: $\sigma_{d}^{2}=40.1 \mu m^{2}$

- The estimate of the variance of the arithmetic mean diameter assuming that only a quarter of droplets are statistically independent: $\sigma_{D_{10}}^{2}=0.059 \mu \mathrm{m}^{2}$

- The true value lies within $\pm 1.96 \sigma_{D_{10}}$ of the arithmetic mean diameter with a $95 \%$ confidence. The resulting AMD and uncertainty is $D_{10}=7.46 \pm .48 \mu \mathrm{m}$

A similar uncertainty analysis approach suggests that the average velocity at this spatial location is $24.4 \pm 1.55 \mathrm{~m} / \mathrm{s}$ which is comparable to the error propagation uncertainty presented in Section 5.4.1. 


\section{Chapter 6}

\section{Results}

\subsection{Data Analysis}

\subsubsection{Injector Dynamics}

The injector needle valve motion is highly dependent on the rate of fuel delivery from the barrel and plunger style injection pump. As discussed in Chapter 2, the injector needle valve opens as fuel pressure surpasses the nozzle body spring pressure. This injection system produces a rapid increase in fuel pressure. Immediately after the opening of the injector needle valve, high pressure fuel is discharged, rapidly reducing the injector sac fuel pressure. This causes the needle valve to close, given that the spring pressure is now slightly higher than the sac pressure. These oscillations can be observed throughout the main injection period from the injector needle lift trace illustrated in Figure 6.2.

The needle lift trace in Figure 6.1 is synchronized to the CCD spray images of Figure 6.2. The first image shows how the initial fuel discharged, as the nozzle opens, is contained on the nozzle axis forming a jet. The next frame 6.2(b) demonstrates how the hollow cone spray develops approaching the maximum needle lift. These findings are in agreement with visualization work performed by Ryan in 1984 [55]. The needle 


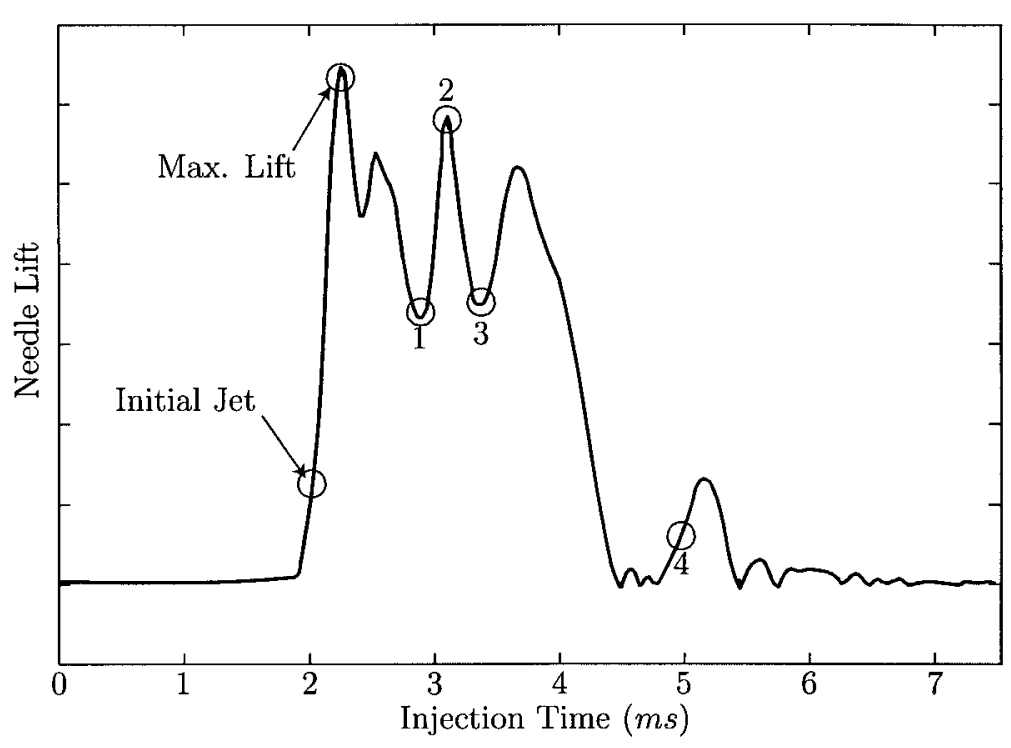

Figure 6.1: Nozzle needle dynamic behaviour

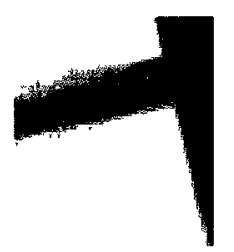

(a) Initial Jet

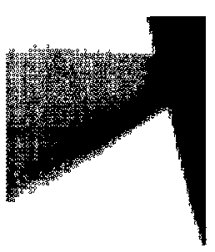

(b) Max lift

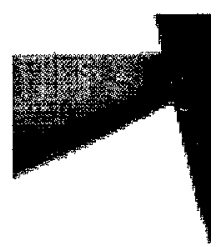

(c) Position 1

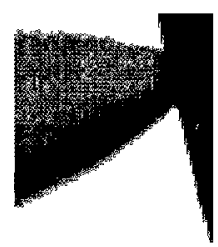

(d) Position 2

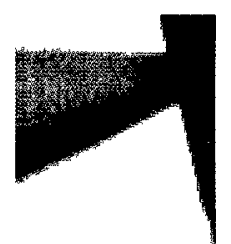

(e) Position 3

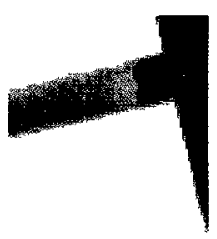

(f) Position 4

Figure 6.2: Spray image synchronized to the needle lift trace

valve oscillations in position 1,2 , and 3 demonstrates how the needle fluctuations affect the hollow cone angle of the spray. The cone angle appears to maximize as the needle position is at a peak of the trace and minimizes at local minima positions. This suggests that droplet size and velocity in the transient region would be expected to fluctuate as the instantaneous fuel injection rate $\dot{m}$ is a function of the pressure 
difference between the injector sac pressure $P_{1}$ and the ambient pressure $P_{2}$ as: [55]

$$
\begin{aligned}
\dot{m} & =C_{d i s} A \sqrt{2 \rho_{\text {fuel }}\left(P_{1}-P_{2}\right)} \\
A & =\text { Instantaneous flow area } \\
C_{d i s} & =\text { Instantaneous discharge coefficient } \\
\rho_{\text {fuel }} & =\text { Density of the liquid fuel injected }
\end{aligned}
$$

Figure 6.2(f) demonstrates how fuel is discharged after the main injection period. These secondary injections are contained to the nozzle axis and are expected to have a larger droplet statistic as the diameter of injected droplets are inversely proportional to the pressure difference $P_{1}-P_{2}$ which is considerably lower compared to the main injection [20]. Secondary injections are caused by pressure waves in the fuel line after the initial injector shutoff. Usually, secondary injections are prevented using a contraction valve installed on the injector body. The IQT ${ }^{\mathrm{TM}}$ 's injection system is not able to be equipped with a contraction valve as the location of this valve on the injector body is currently occupied by the needle lift proximity sensor.

\subsubsection{Analysis Procedure}

Post-processing of all acquired data using the PDA system is accomplished using Matlabß Version 7.1 (Mathworks, Natick, MA). A description of this algorithm and a sample of raw data from the PDA system is included in Appendix D. This allows more control of data analysis and representation. The concatenated unprocessed data for each spatial location from the PDA system is written to a text file. This file now becomes the input of an M-file in Matlab®, which completes the data processing. The text file contains information of all validated droplets. The time of flight, $u$ and $v$ velocities, droplet diameter and the phase difference $\Phi_{12}, \Phi_{13}$ are some of the 
variables available.

It is important to discard all droplets initiating from the secondary injection event as this would not be a truthful representation of the spray characteristics. For this to be accomplished, the post-processing M-file requires knowledge of the time for which the main injection is completed, i.e., the time when the last droplets injected in the main injection reach the measurement volume some distance away from the nozzle tip. This is accomplished by synchronizing a series of high speed images with data collected from the PDA system. The CCD camera is positioned at $30^{\circ}$ in the forward scattering direction and focused on the measurement volume. All frames are then inverted such that scattered light from droplets crossing the measurement volume is represented by dark pixels. Correlation of the time (PDA time) for which the last droplets of the main injection cross the measurement volume is then possible.

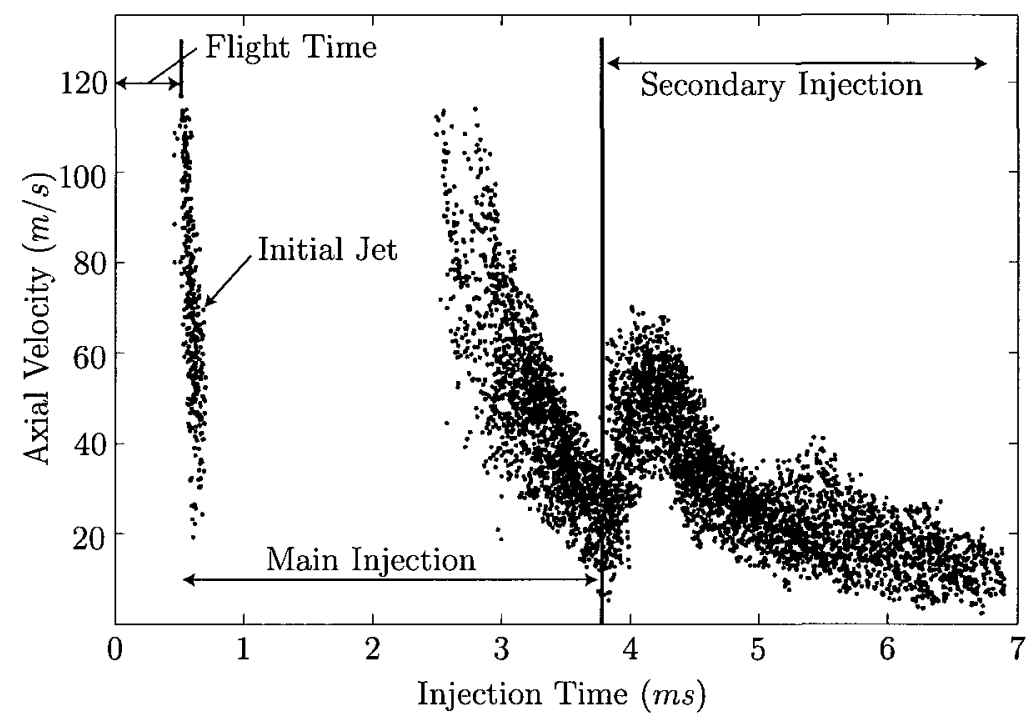

Figure 6.3: Velocity data at $85 \mathrm{~mm}$ from nozzle tip and $0 \mathrm{~mm}$ radial

Figure 6.3 illustrates the axial velocity data collected on the nozzle axis at $x=$ $85 \mathrm{~mm}$ and $r=0 \mathrm{~mm}$ as an example where time $0 \mathrm{~ms}$ corresponds to $t \simeq 2 \mathrm{~ms}$ from Figure 6.1. The droplet flight time identified is approximately $0.7 \mathrm{~ms}$ for the 
faster moving droplets in the initial jet. The initial jet can be identified at $t=0.7 \mathrm{~ms}$ followed by a lack of data between $0.7<t<2.4 \mathrm{~ms}$. Time based PDA results for all measurement locations are available in Appendix B presented in a similar manner to Koo et al. [49]. The complete analysis of all positions is presented in Section 6.2.

$0.7 \mathrm{~ms}$

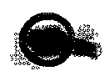

$1.9 \mathrm{~ms}$

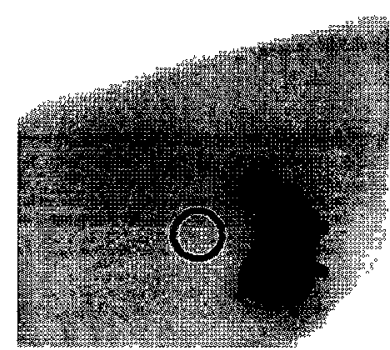

$0.9 \mathrm{~ms}$

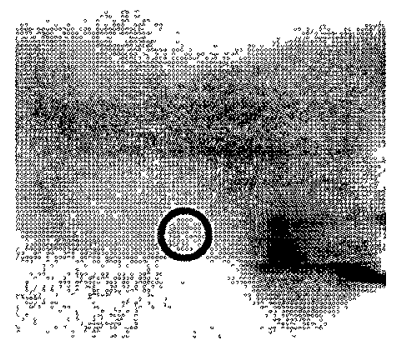

$2.4 \mathrm{~ms}$

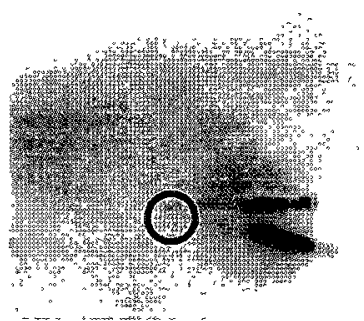

$1.5 \mathrm{~ms}$

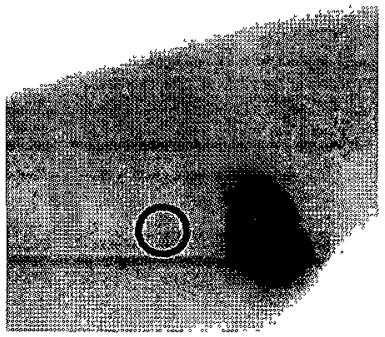

$2.7 m s$

Figure 6.4: Coupling injector needle position to the PDA data at $x=85 \mathrm{~mm}$, $r=0 \mathrm{~mm}$

Initially, it was uncertain if the lack of data between $0.7<t<2.4 \mathrm{~ms}$ was physical. The first hypothesis was that the lack of data could be a result of laser attenuation through the dense spray. However, the high speed images in Figure 6.4 suggest otherwise, where the measurement volume is represented by the circled region. After the initial jet crossing at $0.7 \mathrm{~ms}$, no droplets scatter light until $2.7 \mathrm{~ms}$. This proves that there is actually no droplets present on the nozzle axis after the full hollow cone spray formation. The presence of droplets at $2.7 \mathrm{~ms}$ is the result of the hollow cone closing down after the injector shuts off. 
These findings suggest that droplet velocity and size statistics should be investigated from $0<t<3.6 \mathrm{~ms}$ for the characterization of the main injection at $x=85 \mathrm{~mm}$ for an injected mass of $0.98 \mathrm{~g}$ per 10 injections. The arrival time of secondary injections will be shorter as the measurement volume approaches the nozzle tip. Table 6.1 summarizes the main injection period and position of all measurements performed in this study. The measurement grid was depicted previously in Figure 4.1.

Table 6.1: Measurement position

\begin{tabular}{|l|l|l|l|l|}
\hline Axial Position & $85 \mathrm{~mm}$ & $70 \mathrm{~mm}$ & $55 \mathrm{~mm}$ & $35 \mathrm{~mm}$ \\
\hline Main Injection & $0-3.6 \mathrm{~ms}$ & $0-3.4 \mathrm{~ms}$ & $0-3.2 \mathrm{~ms}$ & $0-3.0 \mathrm{~ms}$ \\
\hline & $0 \mathrm{~mm}$ & $0 \mathrm{~mm}$ & $0 \mathrm{~mm}$ & $0 \mathrm{~mm}$ \\
& $7.5 \mathrm{~mm}$ & $4.5 \mathrm{~mm}$ & $2.5 \mathrm{~mm}$ & $2.0 \mathrm{~mm}$ \\
& $8.5 \mathrm{~mm}$ & $5.5 \mathrm{~mm}$ & $3.5 \mathrm{~mm}$ & $3.0 \mathrm{~mm}$ \\
& $9.5 \mathrm{~mm}$ & $6.5 \mathrm{~mm}$ & $4.5 \mathrm{~mm}$ & $3.5 \mathrm{~mm}$ \\
& $10.5 \mathrm{~mm}$ & $7.5 \mathrm{~mm}$ & $5.5 \mathrm{~mm}$ & $4.0 \mathrm{~mm}$ \\
& $11.5 \mathrm{~mm}$ & $8.5 \mathrm{~mm}$ & $6.5 \mathrm{~mm}$ & $4.5 \mathrm{~mm}$ \\
& $12.5 \mathrm{~mm}$ & $9.5 \mathrm{~mm}$ & $7.5 \mathrm{~mm}$ & $5.5 \mathrm{~mm}$ \\
& $13.5 \mathrm{~mm}$ & $10.5 \mathrm{~mm}$ & $8.5 \mathrm{~mm}$ & $6.0 \mathrm{~mm}$ \\
& $14.5 \mathrm{~mm}$ & $11.5 \mathrm{~mm}$ & & \\
& $15.5 \mathrm{~mm}$ & $12.5 \mathrm{~mm}$ & & \\
& $16.5 \mathrm{~mm}$ & $13.5 \mathrm{~mm}$ & & \\
& & \multicolumn{3}{|l}{} \\
\end{tabular}

In total, 38 positions are interrogated forming a complete measurement set. This measurement set requires 2280 injections to be performed and roughly $230 \mathrm{~g}$ of Viscor calibration fluid. The fluid properties of Viscor are similar to commercial number 2 diesel oil (Refer to Appendix A for the fluid properties of No. 2 diesel oil and Viscor). Three measurement sets were completed for this study. First, a characterization at standard IQT ${ }^{\mathrm{TM}}$ setting is performed. Then, the amount of mass injected per injection cycle was varied in order to investigate the effects of mass injected on 
atomization characteristics. And finally, the injection pump accumulator pressure was increased to investigate the effect of the injection pressure on the atomization performance. These three measurement sets required a total of 6780 injection cycles to be performed.

\subsubsection{Calculated Variables}

The post-processing M-file computes the average axial velocity, arithmetic mean diameter (AMD), Sauter mean diameter (SMD), droplet count and the time resolved axial velocity and diameter based on a specified time step. The average axial velocity and $\mathrm{AMD}$ are found using the following respective equations:

$$
\begin{gathered}
\bar{u}=\frac{1}{N} \sum_{\imath=1}^{N} u_{\imath} \\
D_{10}=\frac{1}{N} \sum_{\imath=1}^{N} D_{\imath}
\end{gathered}
$$

where $N$ is the total validated droplet count in the main injection period.

The most important atomization characteristic for evaporative sprays is the Sauter mean diameter (SMD or $D_{32}$ ). The SMD is a fictitious diameter which represents a mono dispersed droplet size which has the same mass to surface area as the complete droplet size distribution in the spray. The amount of mass to evaporate is a function of $D^{3}$ while the surface area to transfer this mass is a function of $D^{2}$ as:

$$
D_{32}=\frac{\sum_{\imath=1}^{N_{2}} D_{\imath}^{3}}{\sum_{\imath=1}^{N_{\imath}} D_{\imath}^{2}}
$$

\section{Time Resolved Variables}

A more complex representation is used to obtain time resolved variables. The time bin width $(\Delta T)$ is varied in an attempt to find the optimal selection that will fit 
both the axial velocity and diameter of droplets. As $\Delta T$ is reduced, the time resolved data becomes smoother until the point where the lack of data per time bin produces fluctuation in the time resolved data.

Figure 6.5 demonstrates the importance of the $\Delta T$ selection. A $\Delta T$ of $0.1 \mathrm{~ms}$ (Figure 6.5(c) and 6.5(d)) results in a good resolution and representation of the time resolved axial velocity and AMD when compared to $\Delta T=1.0 \mathrm{~ms}$ (Figure 6.5(a) and 6.5(b)). The fluctuations in the time resolved data appears to start at $\Delta T=0.05 \mathrm{~ms}$ (Figure 6.5(f)). These fluctuations are amplified when using smaller bin widths of $0.01 \mathrm{~ms}$ (Figure 6.5(g) and 6.5(h)). These results suggests that the bin width selection for this study should be $0.1 \mathrm{~ms}$. It is important to note that any of these $\Delta T$ 's cannot represent time resolved SMD given that the SMD calculations requires an important amount of data per bin for good statistical representation. Hence, characterization of time resolved SMD was not performed. The time bin width $(\Delta T)$ selected for this study is in agreement with previous work completed by Kim et al. [56] and Arcoumanis et al. [57] where they concluded that a $\Delta T \simeq 0.10 \mathrm{~ms}$ was ideal for transient diesel sprays. The time resolved axial velocity and AMD can be represented by Eq. (6.5). 


$$
\begin{aligned}
\bar{u}(t) & =\sum_{\jmath=1}^{N_{\jmath}} \frac{1}{N_{\imath}} \sum_{\imath=1}^{N_{\imath}} u_{(\Delta t(\jmath-1)<t<\Delta t(\jmath), \imath)} \\
\overline{D_{10}}(t) & =\sum_{\jmath=1}^{N_{\jmath}} \frac{1}{N_{\imath}} \sum_{\imath=1}^{N \imath} D_{(\Delta t(\jmath-1)<t<\Delta t(\jmath), \imath)} \\
\imath & =\text { Particular droplet } \\
j & =\text { Particular time bin } \\
N_{\jmath} & =\text { Number of time bins } \\
N_{\imath} & =\text { Droplet count in bin } \jmath \\
u(t) & =\text { Axial velocity } \\
D(t) & =\text { Droplet diameter }
\end{aligned}
$$



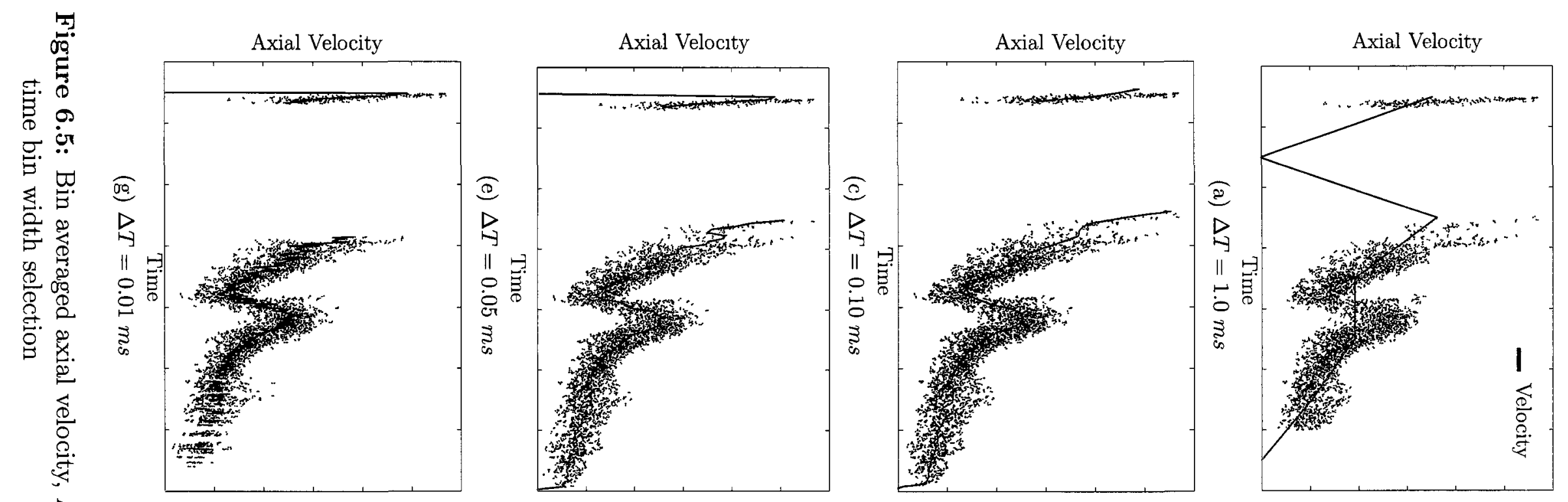

疍
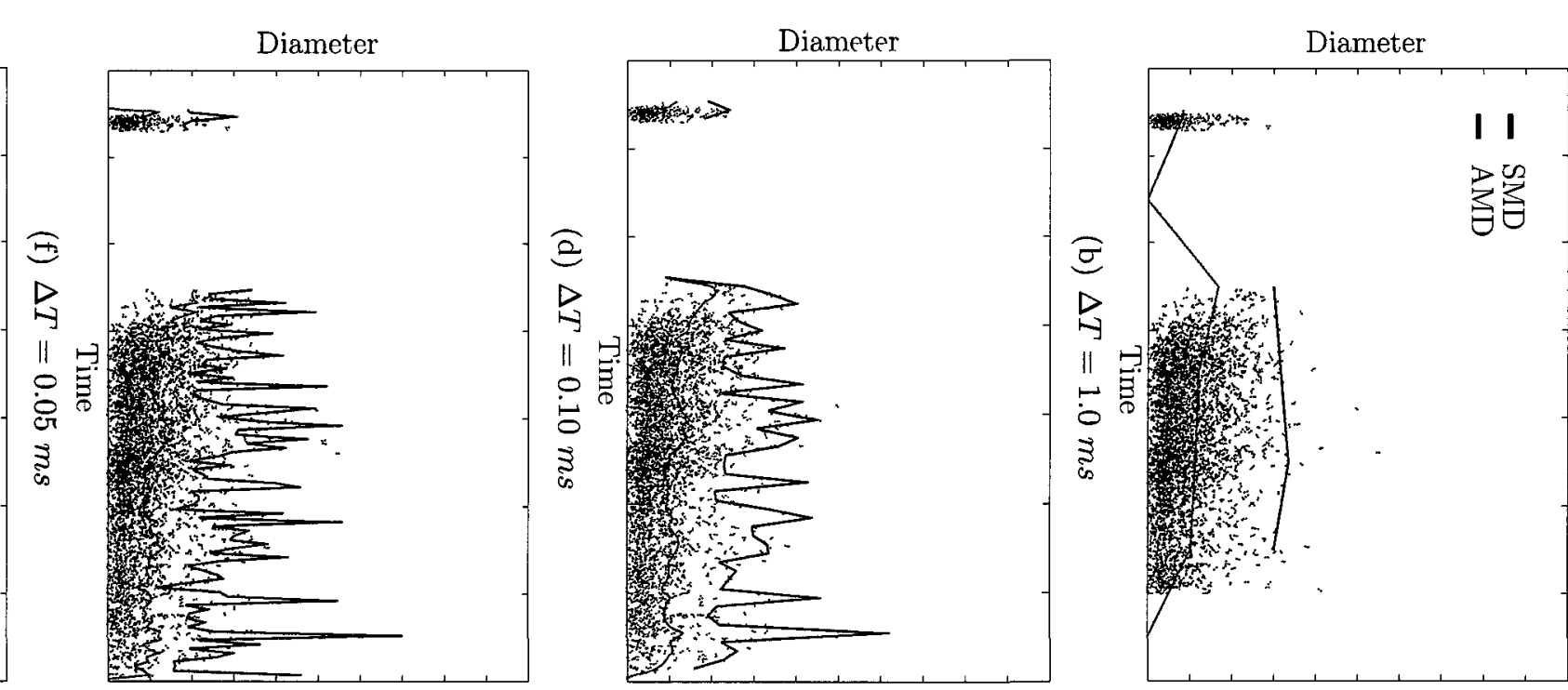


\subsection{Spray Characteristics at Standard IQT ${ }^{\mathrm{TM}}$ Set- ting}

\subsubsection{Qualitative Characteristics from High Speed Imaging}

The first measurement set is performed at standard IQT ${ }^{\mathrm{TM}}$ settings. A mass calibration as per the IQT ${ }^{\mathrm{TM}}$ 's owners manual is accomplished [2]. The injected mass is set at $0.98 \mathrm{~g} / 10$ injections and the air accumulator is charged with a pressure of 175 psi for each injection cycle. A series of spray images are analyzed for the qualitative characterization of the fuel spray. The high speed CCD camera is positioned at $90^{\circ}$ off the spray axis and synchronized with the trigger. Front light photography is used to capture the fuel spray. A background frame is acquired and removed from each of the spray frames in Figure 6.6. The resulting monochromatic frames are then inverted for better representation.

Figure 6.6 illustrates the development of the fuel spray with time steps of $0.5 \mathrm{~ms}$ (2000 fps). The first frame demonstrates the initial jet formation followed by the generation of the hollow cone spray. The initial hollow cone angle is found to be $28.6^{\circ}$ which is quite large compared to the fully developed average hollow cone spray angle $\left(\simeq 13.7^{\circ}\right)$ derived from the spray edge pixel geometry. This large initial angle is in agreement with visualization work completed by Bogin et al. [14]. Evaluating the initial jet velocity is possible knowing the distance traveled from the pixel difference at the jet tip. The pixel difference is found to be roughly $31 \pm 2$ pixels which corresponds to $48 \pm 4 \mathrm{~mm}$ from the calibration image. This corresponds to an axial velocity of $96 \pm 8 \mathrm{~m} / \mathrm{s}$. The fully developed hollow cone angle fluctuates between $11.6^{\circ}$ and $15.0^{\circ}$. 


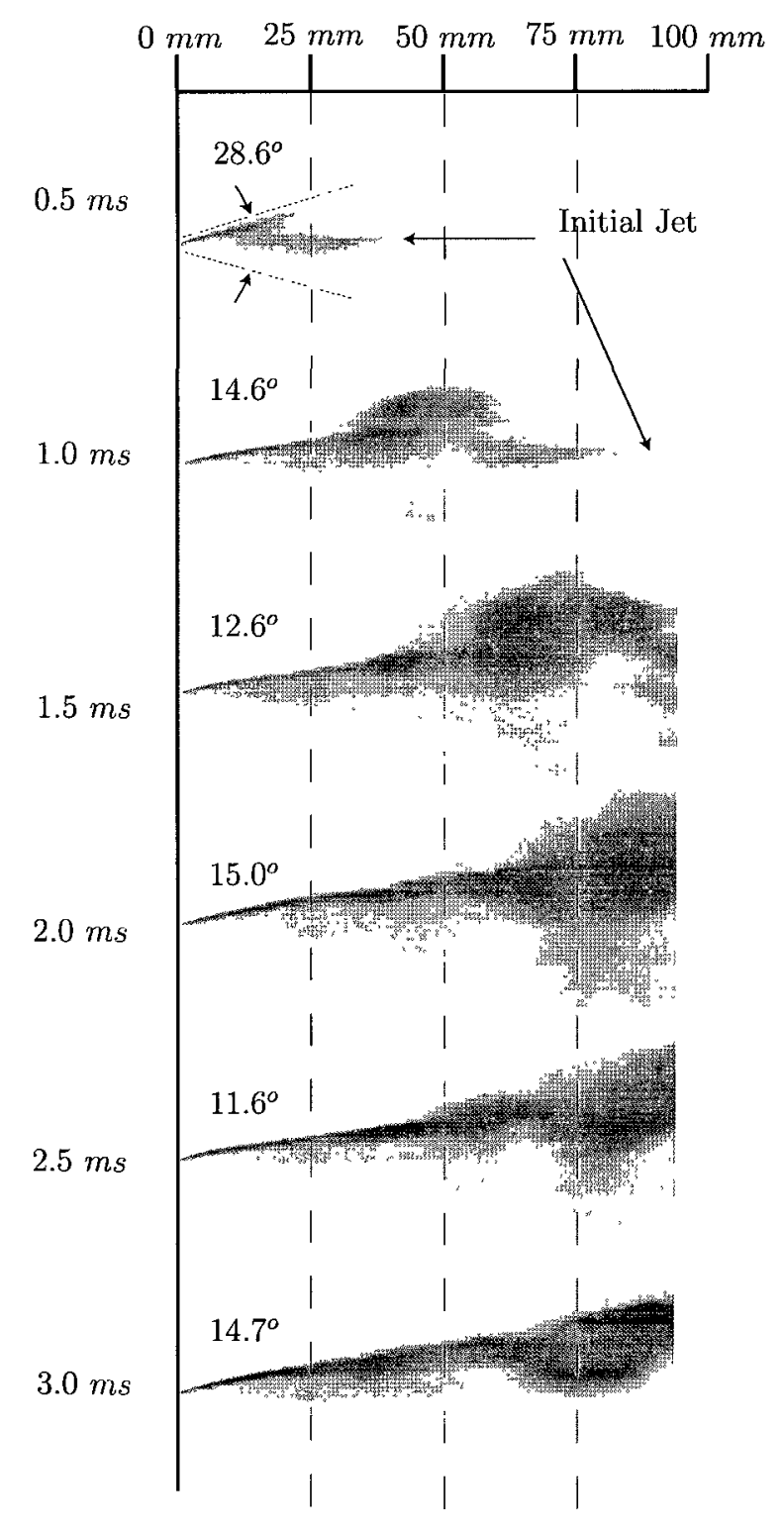

Figure 6.6: Side view of the injection spray in atmospheric ambient air at standard IQT $^{\mathrm{TM}}$ setting 


\subsubsection{Radial Analysis of PDA Measurements}

\section{Axial Velocity}

The axial velocity is averaged over the main injection period for all radial and axial position of Table 6.1. Figure 6.7(a) illustrates the velocity profile as a function of radial position $r$ for three different axial distances. The maximum velocity is located on the spray axis and quickly decreases as the radial distance increases. The axial velocity on the spray axis also decreases as a function of axial distance. This is also expected given that drag force reduces the velocity of droplets. Initially, it was complicated to demonstrate velocity trends from multiple axial positions. Given that the velocity profile of the spray is self-similar, the radial position is divided by the the axial position $x$. This results in a dimensionless approach were the $\mathrm{x}$-axis now represents constant angles as:

$$
r^{\prime}=\frac{r}{x}
$$

while the y axis represents the dimensionless velocity as:

$$
u^{\prime}=\frac{u(x, r)}{u(x, 0)}
$$

where $u(x, 0)$ is the axial velocity on the nozzle axis distance $x$ from the nozzle tip.

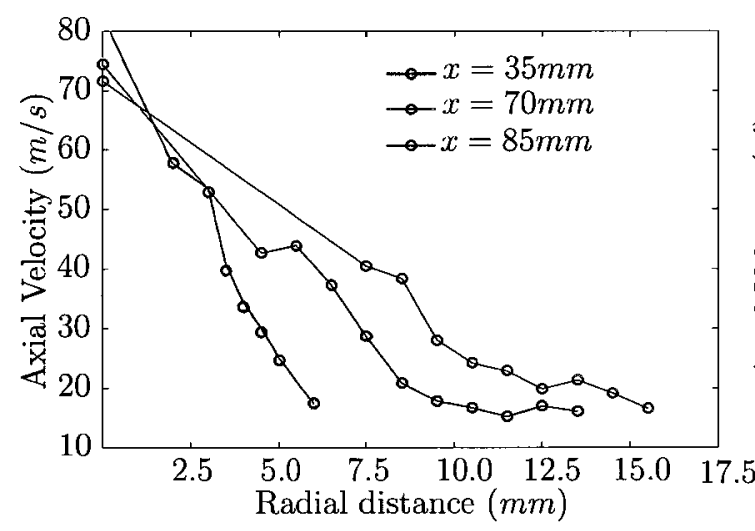

(a) Axial velocity vs radial distance

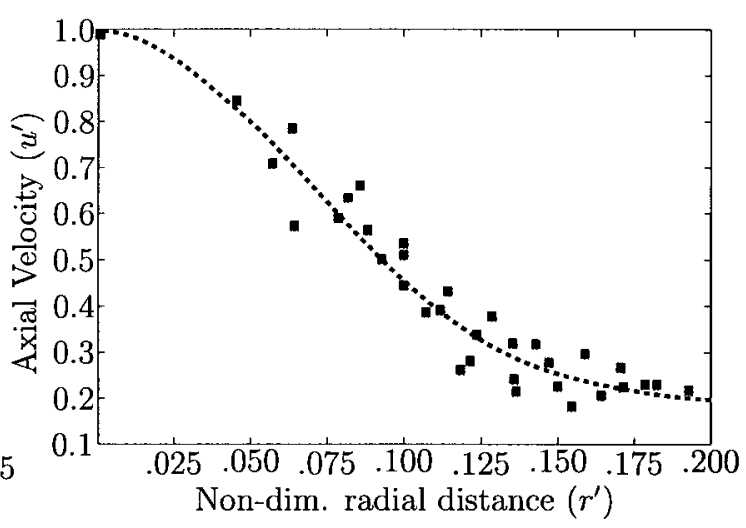

(b) Dimensionless velocity vs radial distance

Figure 6.7: Axial velocity vs radial position 
Figure 6.7(b) demonstrates how the axial velocity decreases with the increase of the angle $\left(\arctan r^{\prime}\right)$ from the nozzle axis. Representing the axial velocity using a dimensionless approach truly demonstrates how the axial velocity decreases as the angle between the interrogation point and the nozzle axis increases. For example, axial velocity at $r^{\prime}=0.10$ which corresponds to a half angle of $5.71^{\circ}$ is $0.4 u_{o}$. This suggest that for this angle, the axial velocity is expected to be $0.4 u_{o}$ where $u_{o}$ is the axial velocity $u(x, 0)$.

\section{Diameter Statistics and Droplet Count}

The next spray characteristic studied is the diameter statistics as a function of the nondimensional radial position. Along with the diameter statistics, droplet count is also investigated. Figure 6.8 demonstrates how the droplet AMD changes with $r^{\prime}$ but more importantly, how the particle count appears Gaussian distributed and centered in the middle of the hollow cone spray. The droplet count is nondimensionalized by the maximum particle count for every axial position.

Most large droplets are located inside the hollow cone spray were the droplet count is relatively low $\left(0<r^{\prime}<0.05\right)$. This larger droplet size statistic is a result of the initial jet after the SOI. The AMD on the nozzle axis is roughly $13.5 \mu \mathrm{m}$. The total droplet count located on the axis is only $20 \%$ of the maximum droplet count which is located at $r=0.11$. The AMD at the maximum count location is $8.0 \mu \mathrm{m}$, which is significantly smaller than the axis AMD. The AMD decreases to a minimum value of $7.5 \mu m$ inside the spray periphery at $r^{\prime}=0.15$. The weighted average of droplet size at standard IQT ${ }^{\mathrm{TM}}$ setting is found knowing the diameter and count for all measurement points. The weighted average of the AMD and SMD over 73531 droplets is $8.79 \mu \mathrm{m}$ and $26.29 \mu \mathrm{m}$ respectively. Table 6.2 is a summary of the droplet size statistics at all axial locations with their respective uncertainty derived using Section 5.4. 
Table 6.2: Result summary, standard injection parameters. Droplet size uncertainty found using analysis in Section 5.4.2.

\begin{tabular}{|c|c|c|c|c|c|}
\hline$x \mathrm{~mm}$ & $\mathrm{AMD} \mu \mathrm{m}$ & $\sigma_{d} \mu m^{2}$ & Count & Uncertainty $\mu m$ & SMD $\mu m$ \\
\hline 35 & 9.32 & 8.22 & 15331 & \pm 0.18 & 29.41 \\
55 & 9.88 & 7.62 & 12171 & \pm 0.19 & 25.15 \\
70 & 8.56 & 7.36 & 23070 & \pm 0.13 & 26.49 \\
85 & 8.27 & 7.04 & 22413 & \pm 0.13 & 25.09 \\
\hline
\end{tabular}

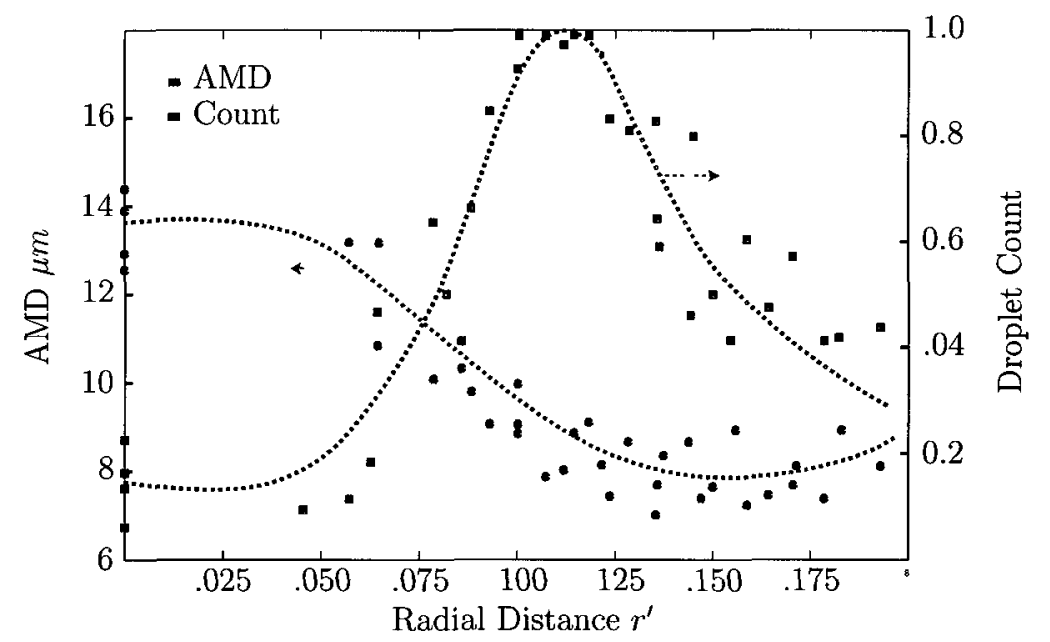

Figure 6.8: AMD and dimensionless droplet count as a function of nondimensional radial position $r^{\prime}$

The nondimensional droplet count also indicates the angle of the hollow cone spray. As discussed earlier, the nondimensional radial distance can be viewed as constant angles from the nozzle axis. The hollow cone spray half angle derived from the maximum count location of $r^{\prime}=0.110$ is $6.28^{\circ}\left(12.56^{\circ}\right.$ full angle $)$. This results is surprisingly similar to the spray angle found from high speed imaging where the spray angles varied from $11.6^{\circ}$ to $15.0^{\circ}$. The small count of droplets located at $r^{\prime}=0.20$ which corresponds to a half angle of $11.31^{\circ}$ is likely the result of the large initial angle found from high speed imaging illustrated in frame $0.5 \mathrm{~ms}$ of Figure 6.4. 


\subsubsection{Time Resolved Analysis}

Given the highly transient nature of the spray, time resolved velocity analysis provides a better representation of the spray characteristics. This time resolved analysis provides the average droplet velocity for each specified time bin as a function of spatial position. In order to represent the development of the spray, a custom Matlab® Mfile was written to output the bin averaged velocity of all axial positions investigated. The radial positions are mirrored over the nozzle axis for better spray representation.

Figure 6.9 demonstrates the velocity profile of all four axial positions investigated as a function of time. The initial jet can be identified at the $35 \mathrm{~mm}$ location in Figure 6.9(a). The jet is concentrated to the nozzle axis supporting findings from the CCD high speed images and work completed by Ryan [55]. The following axial positions have not yet registered any droplets at this time step.

In the following frame (Figure 6.9(b)), the initial jet now reaches the $70 \mathrm{~mm}$ location. The central jet can no longer be identified at $35 \mathrm{~mm}$ indicating the start of the hollow cone spray at this position. The hollow cone forms an initial full angle of approximately $22.6^{\circ}$. This angle is smaller then the initial angle reported by Bogin et al. on the IQT ${ }^{\mathrm{TM}}$ [14]. The visualization work by this author suggests an initial spray angle of $35^{\circ}$. The discrepancy between the two studies is a result of the differences in fluid properties of the injected fuels. Bogin et al. characterized the IQT spray using n-heptane which has a density and viscosity of $0.684 \mathrm{~g} / \mathrm{ml}$ and $0.56 \mathrm{cst}$, respectively, which is considerably less viscous than Viscor, which is used in this study.

The hollow cone fully develops by frame $6.9(\mathrm{c})$ where velocity oscillations can be identified from frame $6.9(\mathrm{~d})$ to $6.10(\mathrm{~b})$. The magnitude of the axial velocity changes, however, the profile shape remains somewhat constant throughout the main injection. 
The magnitude of these oscillations in droplet velocity are the result of the highly unsteady pintle dynamics discussed earlier in Section 6.1.1.

The injector is then fully closed by Figure $6.10(\mathrm{c})$ this causes the hollow cone to collapses at the $35 \mathrm{~mm}$ position. Given the droplet flight time, the hollow cone collapse at the remaining locations in the following frame.

The fully develop hollow cone spray profile suggests higher velocities towards the nozzle axis for all axial positions. This is caused by the entrainment of air from outside the hollow cone spray. The largest velocity gradient is found between the stagnant air and the fuel droplets on the outer periphery of the hollow cone spray. This produces high shear stresses accelerating the stagnant air by momentum transfer. This in fact reduces the velocity of droplets on the outside spray periphery. These findings are in agreement with spray characterization of a piezo pintle type injector performed by Nouri et al. [58]. A series of figures with a resolution of $0.1 \mathrm{~ms}$ from 0 to $4.4 \mathrm{~ms}$ is available in Appendix C. 


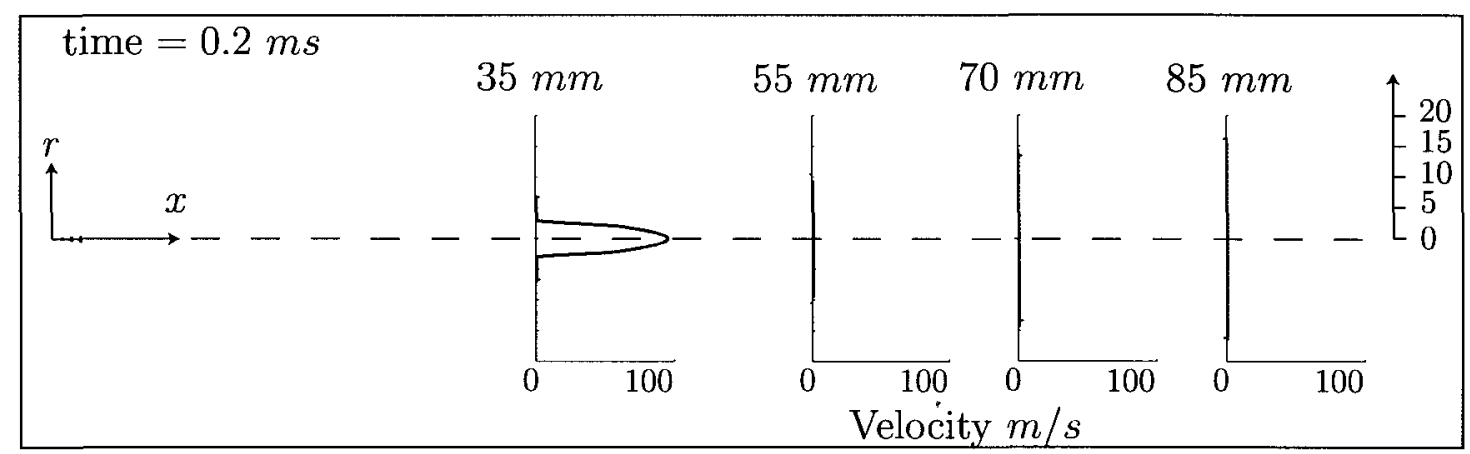

(a) time $=0.2 \mathrm{~ms}$

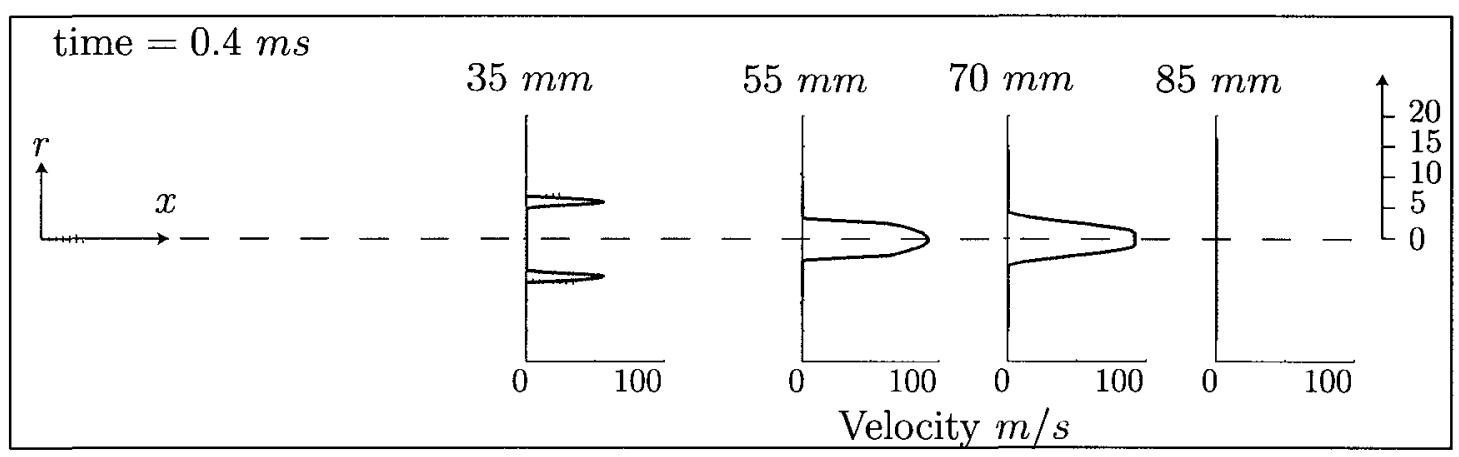

(b) time $=0.4 \mathrm{~ms}$

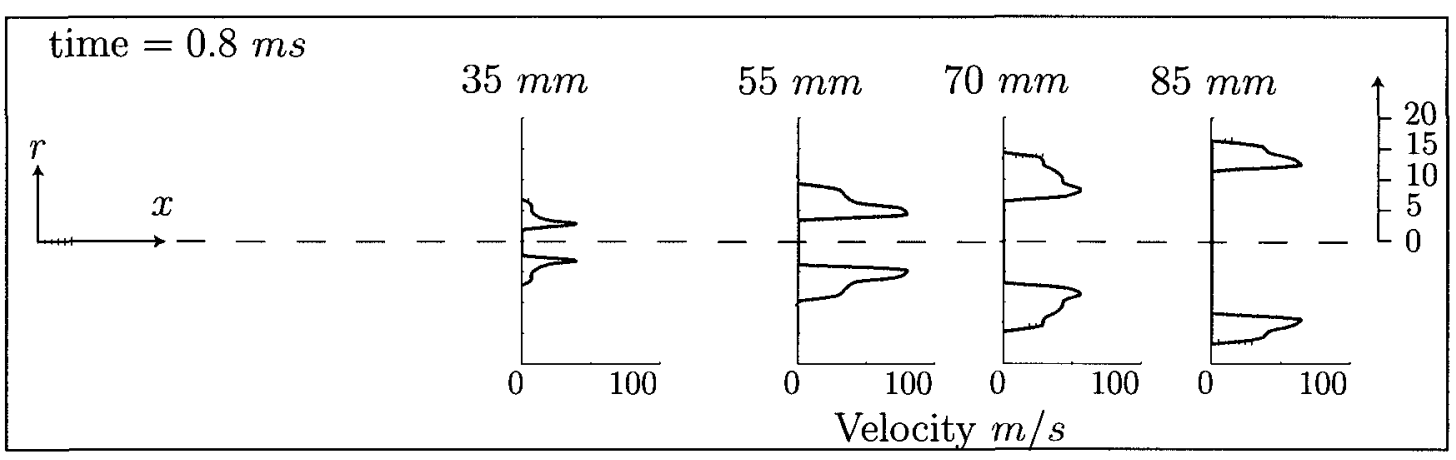

(c) time $=0.8 \mathrm{~ms}$

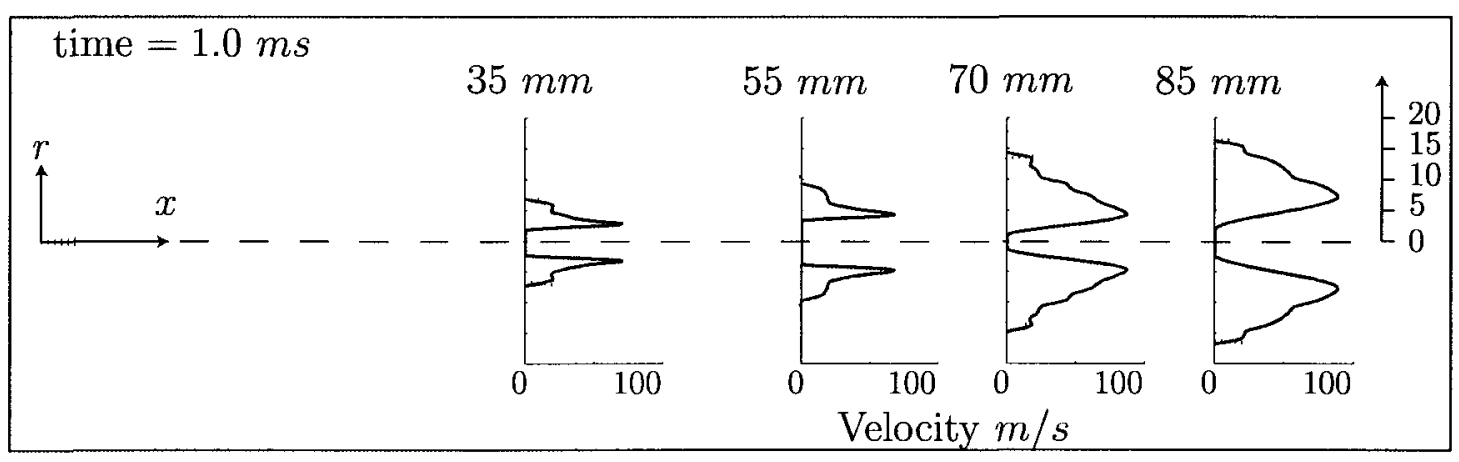

(d) time $=1.0 \mathrm{~ms}$

Figure 6.9: Velocity profiles $0.2 \mathrm{~ms}$ to $1.0 \mathrm{~ms}$ 


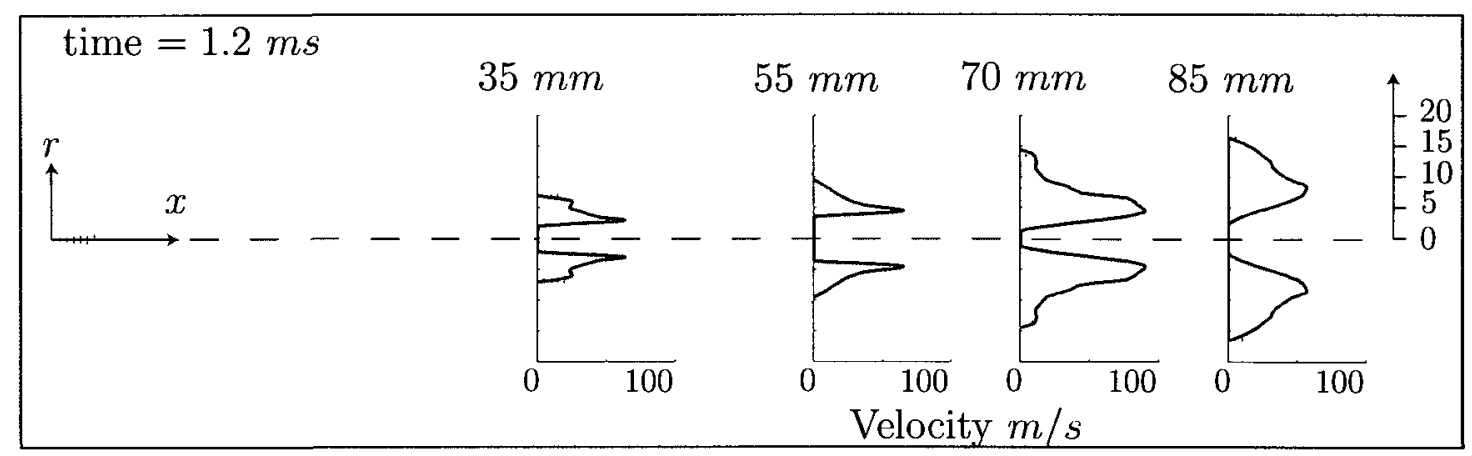

(a) time $=1.2 \mathrm{~ms}$

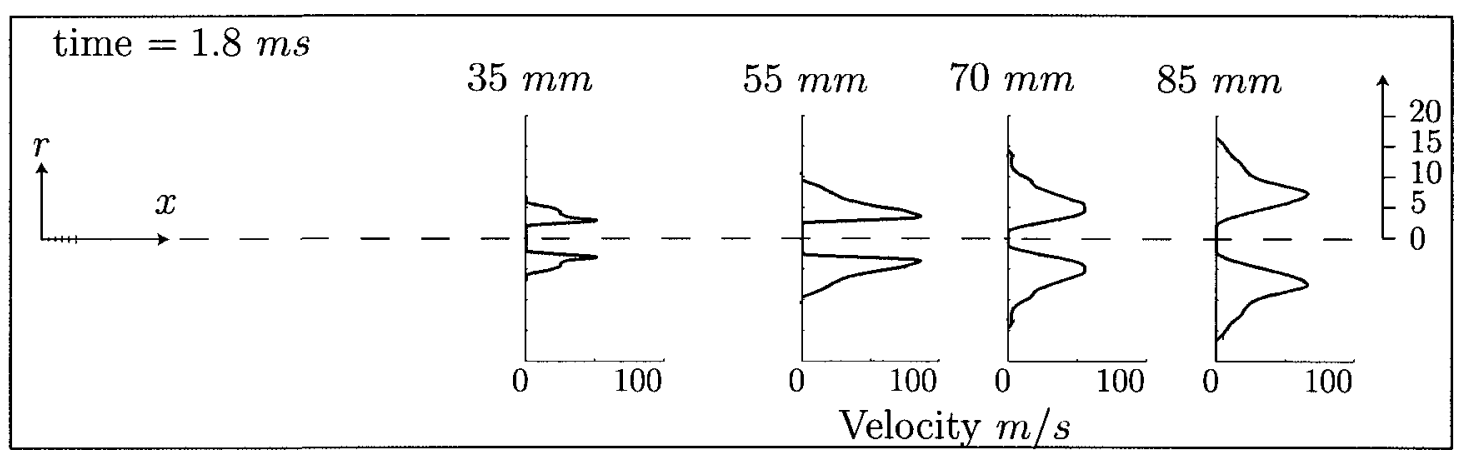

(b) time $=1.8 \mathrm{~ms}$

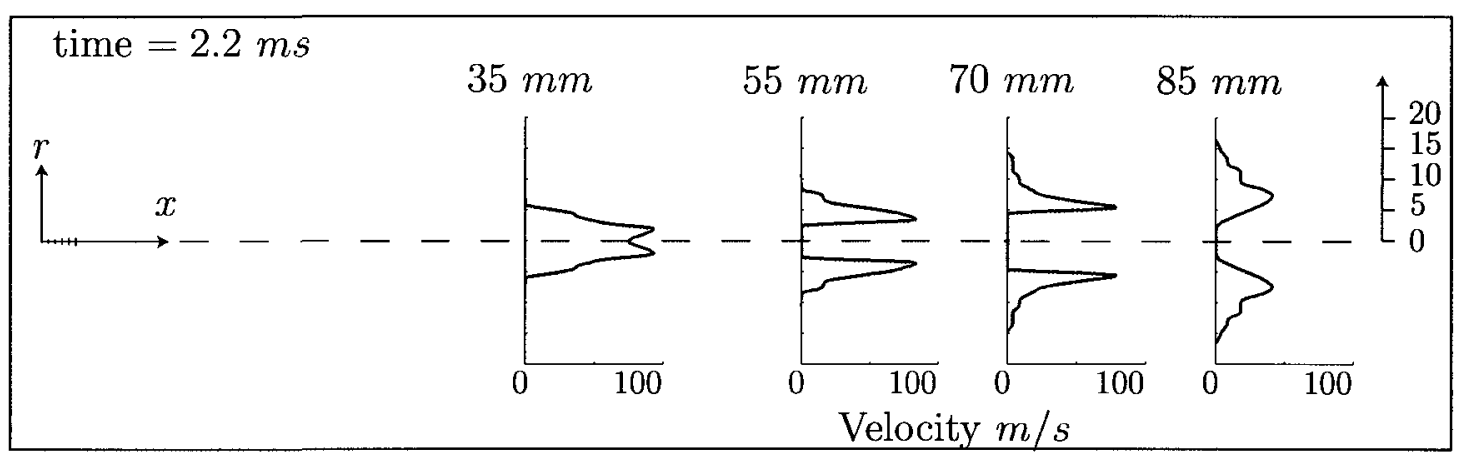

(c) time $=2.2 \mathrm{~ms}$

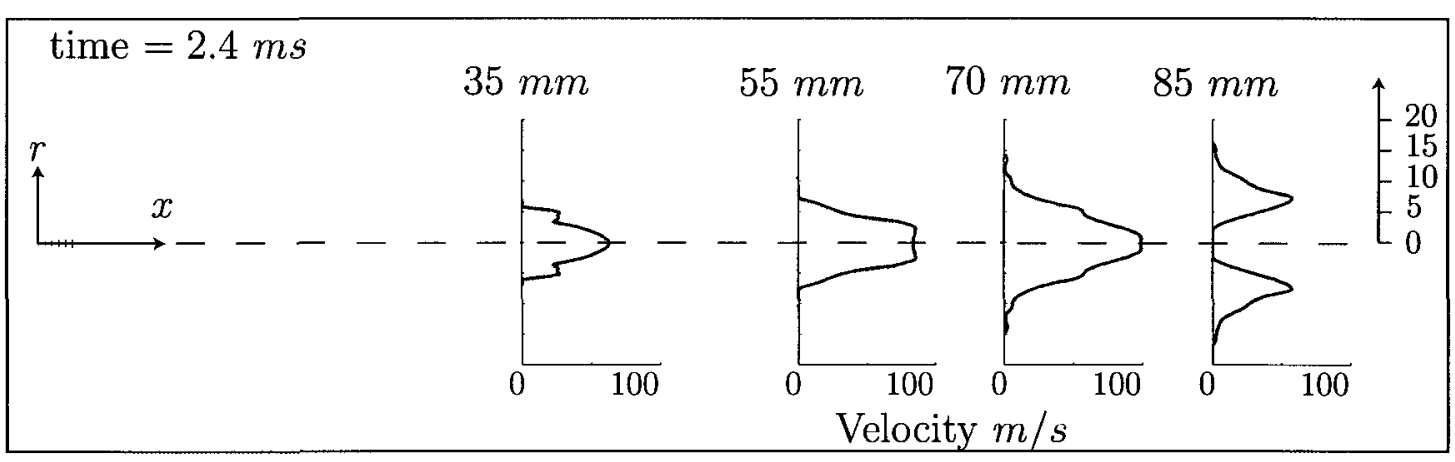

(d) time $=2.4 \mathrm{~ms}$

Figure 6.10: Velocity profiles $1.2 \mathrm{~ms}$ to $2.4 \mathrm{~ms}$ 
The main injection can be separated into three periods. The initial jet and hollow cone formation which occurs between $(0<t<0.7 \mathrm{~ms})$ followed by the fully developed injection period $(0.7<t<1.8 \mathrm{~ms})$ and finally, the hollow cone collapse period $(1.9<$ $t<3.0 \mathrm{~ms})$. The most steady state behaviour of the spray is found in the fully developed injection period. Spray characteristics in this period are further analyzed in order to identify the fluctuation as a function of the nondimensional radial position.

Figure 6.11 demonstrates the unsteadiness of the spray even in the most steady state injection period. The root-mean-square (RMS) of the axial velocity at $x=35 \mathrm{~mm}$ is largest at the inner periphery of the hollow cone and decreases towards the outer periphery of the cone. Comparing the RMS to the average velocity. The lowest RMS result is $\sigma=9.2 \mathrm{~m} / \mathrm{s}$ located at the outer spray periphery where the average velocity is approximately $20 \mathrm{~m} / \mathrm{s}$.

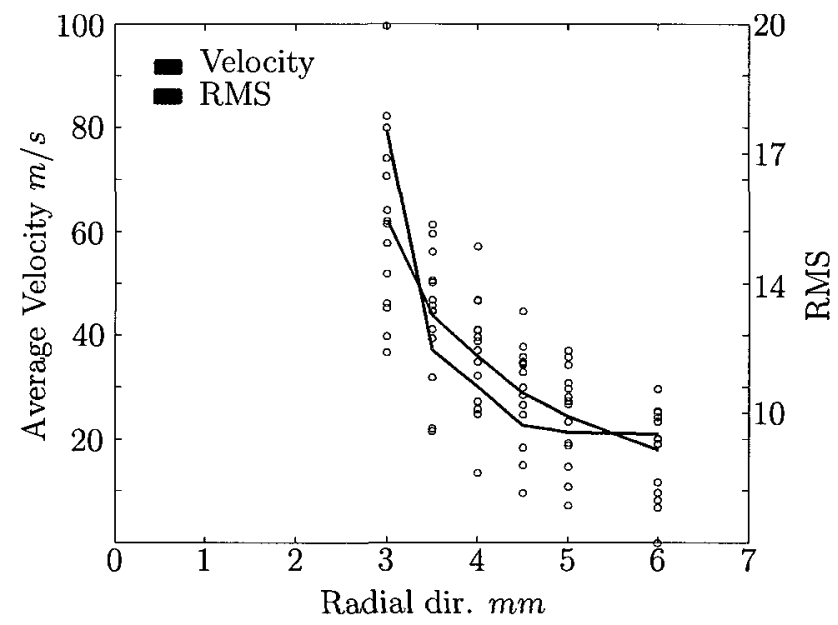

Figure 6.11: Velocity RMS at $x=35 \mathrm{~mm}$

\subsection{Effect of Mass Injected on Atomization}

The injected mass is lowered in order to investigate the effect of mass injected on atomization characteristics. The pump block is shimmed according to the 
IQT $^{\mathrm{TM}}$ owner's manual [2]. The mass is lowered from $0.98 \mathrm{~g}$ per 10 injections to $0.82 \mathrm{~g}$ per 10 injections. The process of identifying the secondary injection for all axial positions is performed as per Section 6.1.2. Figure 6.12(a) demonstrates the difference in the injector needle traces for the two cases. The total main injection time decreases from $2.4 \mathrm{~ms}$ to $2.0 \mathrm{~ms}$. The secondary injections appear to have larger contributions to the total mass injected as the mass is lowered. This can be identified by the area under the needle trace after $4.0 \mathrm{~ms}$ for the $0.82 \mathrm{~g}$ per 10 injections mass compared to the area after $4.4 \mathrm{~ms}$ for the $0.98 \mathrm{~g}$ per 10 injections mass in Figure 6.12(a). Another important finding from the needle trace comparison is the local minima reduction as the injected mass is lowered. This results in larger oscillations of the hollow cone spray as discussed in Section 6.1.1. Figure 6.12(b) supports this finding as the droplet count is maximum at $r^{\prime}=.136$ which corresponds to a hollow cone half angle of $7.74^{\circ}$ compared to $6.28^{\circ}$ for the larger injected mass.

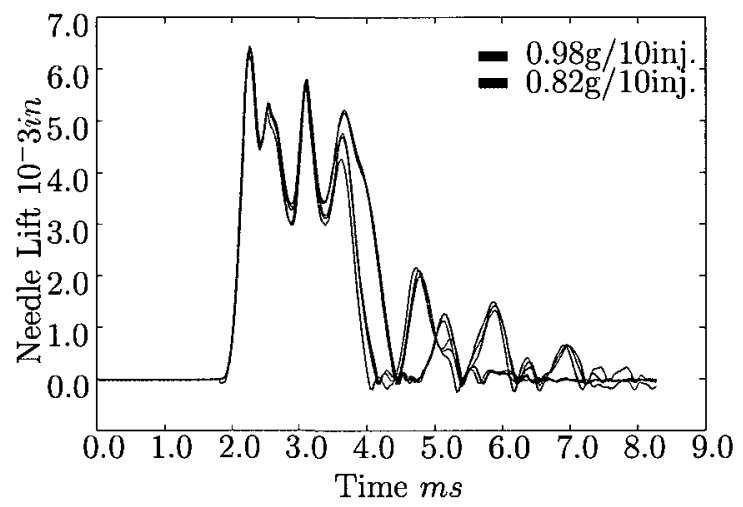

(a)

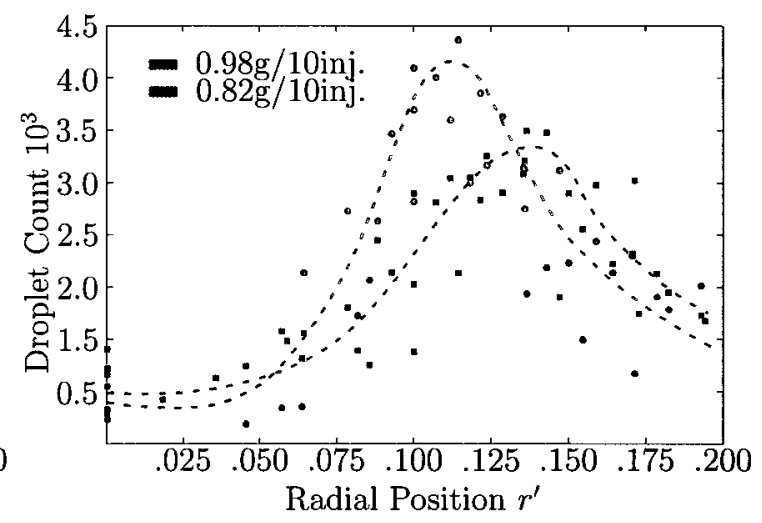

(b)

Figure 6.12: Effect of mass injected on the needle trace and droplet count

The injection pressure for this comparison is kept constant by controlling the accumulator air pressure at 175 psi. However, the energy density per injected mass increases as the injected volume decreased by $16 \%$. This explains the larger axial velocity for the lower mass injected as illustrated in Figure 6.13(a). Higher velocities 


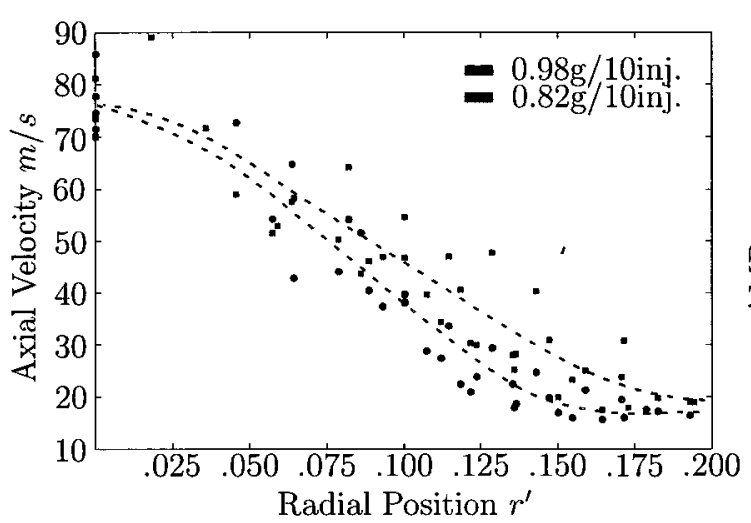

(a)

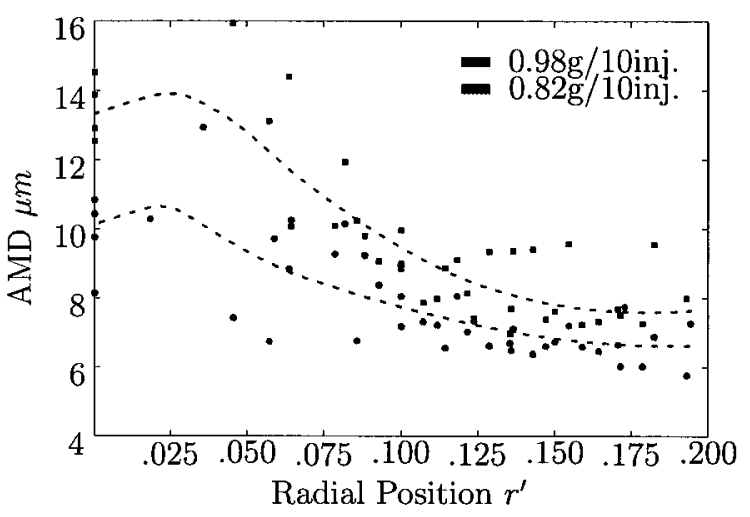

(b)

Figure 6.13: Effect of mass injected on the axial velocity and AMD

lead to larger Weber number suggesting better atomization and smaller droplet diameters which explains the decrease in the AMD for the lower mass injected represented in Figure 6.13(b).

The SMD is also found using the droplet statistics of the entire measurement set for each distinct axial position investigated. Although the AMD decreases as the injected mass is lowered, the opposite is found for the SMD (Table 6.3). The only way this can be physically possible is that droplets are more dispersed as mass is lowered. Dispersity is a nondimensional number that quantifies the uniformity of droplets as:

$$
\sigma_{g}=\sqrt{d_{2} / d_{1}}
$$

Where $d_{1}$ and $d_{2}$ are defined as the value for which $15.9 \%$ of droplets are smaller than $d_{1}$ and larger than $d_{2}$. As the droplet distribution becomes tighter, the dispersity will converge towards unity [36]. The distribution is then called monodisperse. The droplet size distributions for diesel sprays are usually polydisperse with larger $\sigma_{g}$ values [59]. Table 6.3 demonstrates how dispersity $\sigma_{g}$ increases as the injected mass is lowered. The average dispersity for the $0.98 \mathrm{~g}$ per 10 injections and the $0.82 \mathrm{~g}$ per 10 injections is 2.83 and 3.08 , respectively. 
Table 6.3: Result summary, effect of mass injected

\begin{tabular}{|c|c|c|c|c|c|c|}
\hline & \multicolumn{3}{|c|}{$0.98 \mathrm{~g} / 10 \mathrm{inj}}$. & \multicolumn{3}{c|}{$0.82 \mathrm{~g} / 10 \mathrm{inj}}$. \\
\hline \hline$x \mathrm{~mm}$ & AMD $\mu m$ & SMD $\mu m$ & $\sigma_{g}$ & AMD $\mu m$ & SMD $\mu m$ & $\sigma_{g}$ \\
\hline 35 & 9.32 & 29.41 & 2.713 & 6.64 & 32.33 & 3.039 \\
55 & 9.88 & 25.15 & 2.890 & 8.09 & 27.49 & 3.193 \\
70 & 8.56 & 26.49 & 2.784 & 7.51 & 29.41 & 3.046 \\
85 & 8.27 & 25.09 & 2.864 & 7.52 & 24.35 & 3.026 \\
\hline
\end{tabular}

The larger droplet size dispersion of the spray can be explained from the nozzle needle trace. As the injected mass is lowered, the local minima decreases significantly. This increase in needle oscillation causes larger spray angle oscillations and larger droplet dispersions given that the pressure difference between the fuel and ambient air is lower in the minima when compared to the standard mass injected yielding larger droplets.

\subsection{Effect of the Accumulator Pressure on Atom- ization}

The injection energy is increased to investigate its effect on atomization characteristics. The mass injected is kept constant at $0.82 \mathrm{~g}$ per 10 injections while the injection accumulator pressure is increased from 175 psi to 250 psi. This results in a $43 \%$ increase in energy. This pressure increase has a considerable effect on the needle dynamics as illustrated in Figure 6.14(a). The needle initially opens to $7.0 \mathrm{~mm}$ compared to $6.2 \mathrm{~mm}$ for the standard pressure injection. The overall injector needle trace suggests larger needle lift throughout the main injection period as injection pressure is increased. This results in larger fuel pressure as the spring pressure increases linearly with the injector needle lift. 
The location of the maximum particle count in Figure 6.14(b) is very similar suggesting that the average hollow cone angle remains the same for the two cases. However, hollow cone angle fluctuations are expected to be reduced as the injection pressure is increased since the oscillations of the injector needle throughout the main injection are greatly reduced.

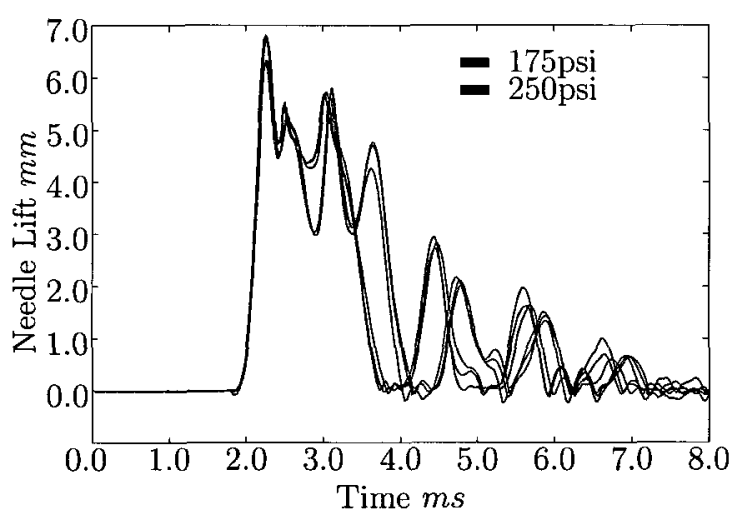

(a)

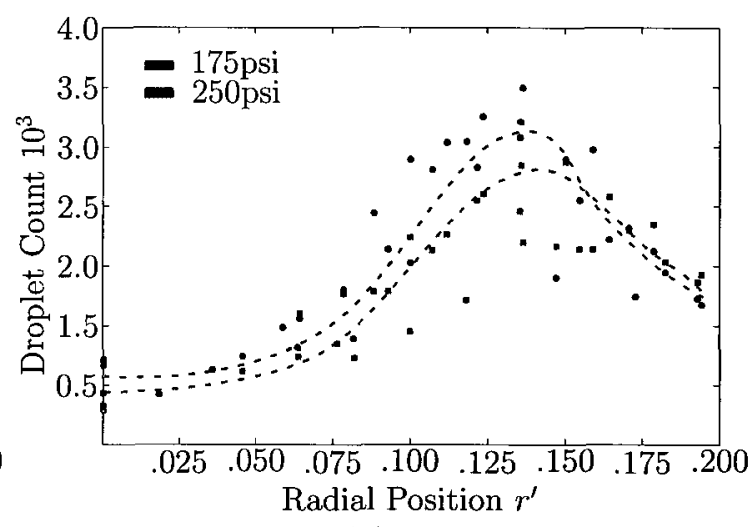

(b)

Figure 6.14: Effect of injection pressure on the needle lift and droplet count

The AMD is found to decrease as the injection pressure increases. Knowing that fuel pressure increases during the main injection period, these findings are in agreement with work completed by Allocca et al. [24] where they concluded that doubling the injection pressure from $10 \mathrm{MPA}$ to $20 \mathrm{MPa}$ resulted in a $28 \%$ reduction of the AMD. The axial velocity, however, did not appear to change between the two cases (Figure 6.15(a)). The equation of motion for non-evaporative, non-buoyant droplets, can be represented by equating the drag force $F_{d}$ to the particle acceleration as:

$$
F_{d}=m \frac{d v}{d t}
$$

Given that the ambient air is stagnant, the relative velocity to the droplet becomes $-v_{p}$. The drag force can then be represented as:

$$
F_{d}=-C_{D} \frac{\rho_{a i r} \pi d^{2}}{8} v_{p}^{2}
$$


Substituting Eq. (6.10) into Eq. (6.9) yields a differential equation for the droplet motion:

$$
\begin{aligned}
\frac{d v}{d t} & =-\frac{3 C_{D}}{4 d} \frac{\rho_{a r r}}{\rho_{\text {fuel }}} \cdot v_{p}^{2} \\
\rho_{\text {air }} & =\text { Density of the air } \\
\rho_{\text {fuel }} & =\text { Density of the liquid fuel } \\
C_{D} & =\text { Drag coefficient of droplet } \\
d & =\text { Droplet diameter } \\
v_{p} & =\text { Droplet velocity }
\end{aligned}
$$

Eq. (6.11) demonstrates how the rate at which velocity decreases is a function of not only the initial droplet velocity but also the diameter. Figure 6.15(a) suggest similar axial velocity downstream of the nozzle for the two cases. Figure 6.15(b) demonstrates that the injector produces smaller droplet diameter as the injection pressure increases. As the droplet diameter decreases, the rate at which the droplet decelerates will increase. Therefore, backtracking velocity to the nozzle tip would suggest that the initial discharge velocity is in fact greater as the injection accumulator pressure is increased.

Table 6.4: Result summary, effect of injection pressure

\begin{tabular}{|c|c|c|c|c|c|c|}
\hline & \multicolumn{2}{|c|}{250 psi injection energy } & \multicolumn{3}{|c|}{175 psi injection energy } \\
\hline \hline$x m m$ & AMD $\mu m$ & SMD $\mu m$ & $\sigma_{g}$ & AMD $\mu m$ & SMD $\mu m$ & $\sigma_{g}$ \\
\hline 70 & 6.70 & 23.94 & 2.880 & 7.51 & 29.41 & 3.046 \\
85 & 6.09 & 22.78 & 2.864 & 7.52 & 24.35 & 3.026 \\
\hline
\end{tabular}




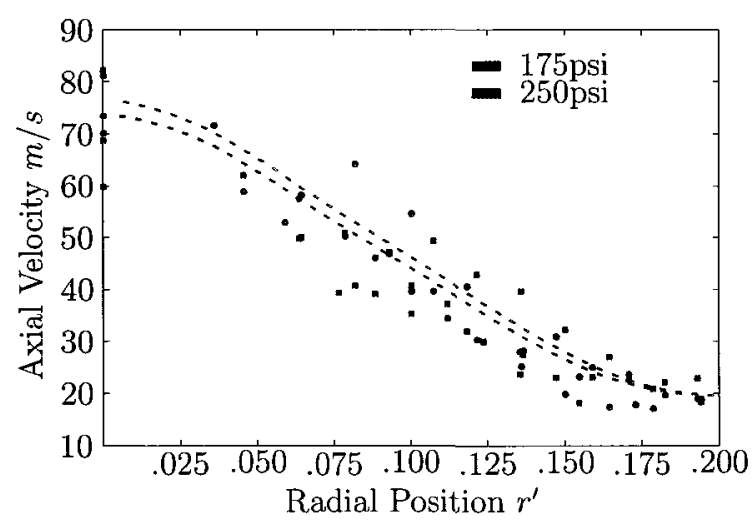

(a)

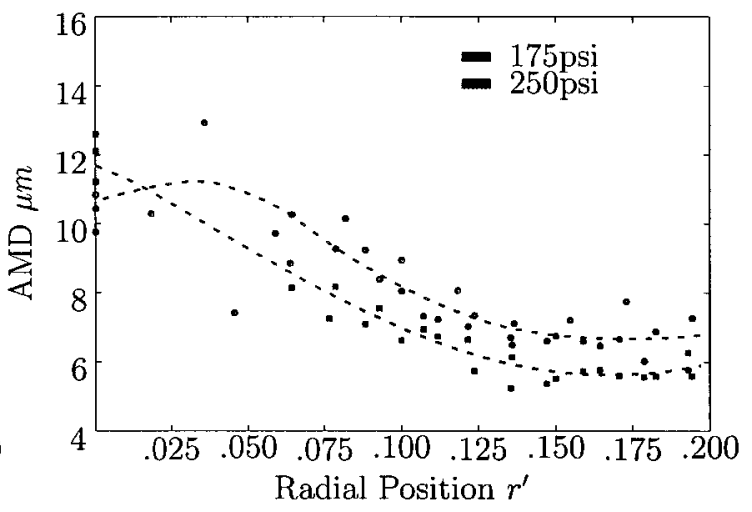

(b)

Figure 6.15: Effect of injection pressure on the axial velocity and AMD

The results from Table 6.4 demonstrates how the AMD and SMD decreases as the injection pressure is increased.

\subsection{Effect of ambient pressure on spray tip pene- tration}

The PDA characterization work completed in this study is performed under atmospheric ambient pressure conditions. The spray tip velocity and penetration as a function of ambient pressure is investigated to correlate the atmospheric PDA results to $\mathrm{IQT}^{\mathrm{TM}}$ operating ambient pressures. The penetration study is accomplished by injecting fuel from 1 to 20 bars in a custom designed combustion chamber of identical IQT $^{\mathrm{TM}}$ dimensions illustrated in Figure 6.16.

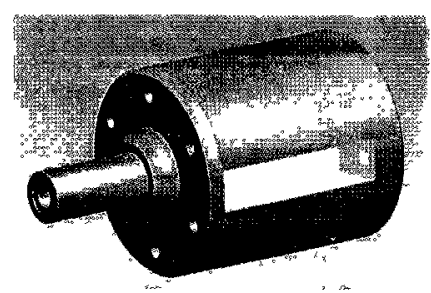

Chamber Assembly

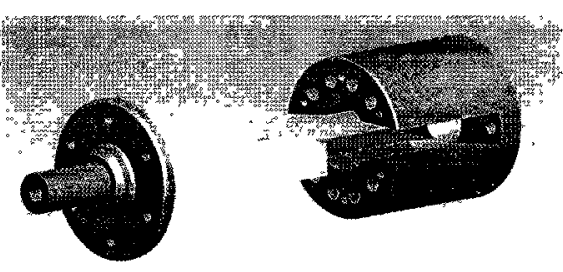

Exploded View

Figure 6.16: Optical chamber 
The chamber is fitted with two optical windows allowing the CCD camera to capture the injection spray using a back lit photography technique. Using pixel counting, the spray tip velocity is determined knowing the frame rate of the acquisition. Pixel counting is not an accurate method of determining the tip velocity $\left(U_{t \iota p}\right)$, the associated error on $U_{t \imath p}$ is roughly $\pm 5 \mathrm{~m} / \mathrm{s}$. Table 6.5 shows results of $U_{t \imath p}$ and the resulting dimensionless velocity reduction as a function of ambient pressure.

1 ba

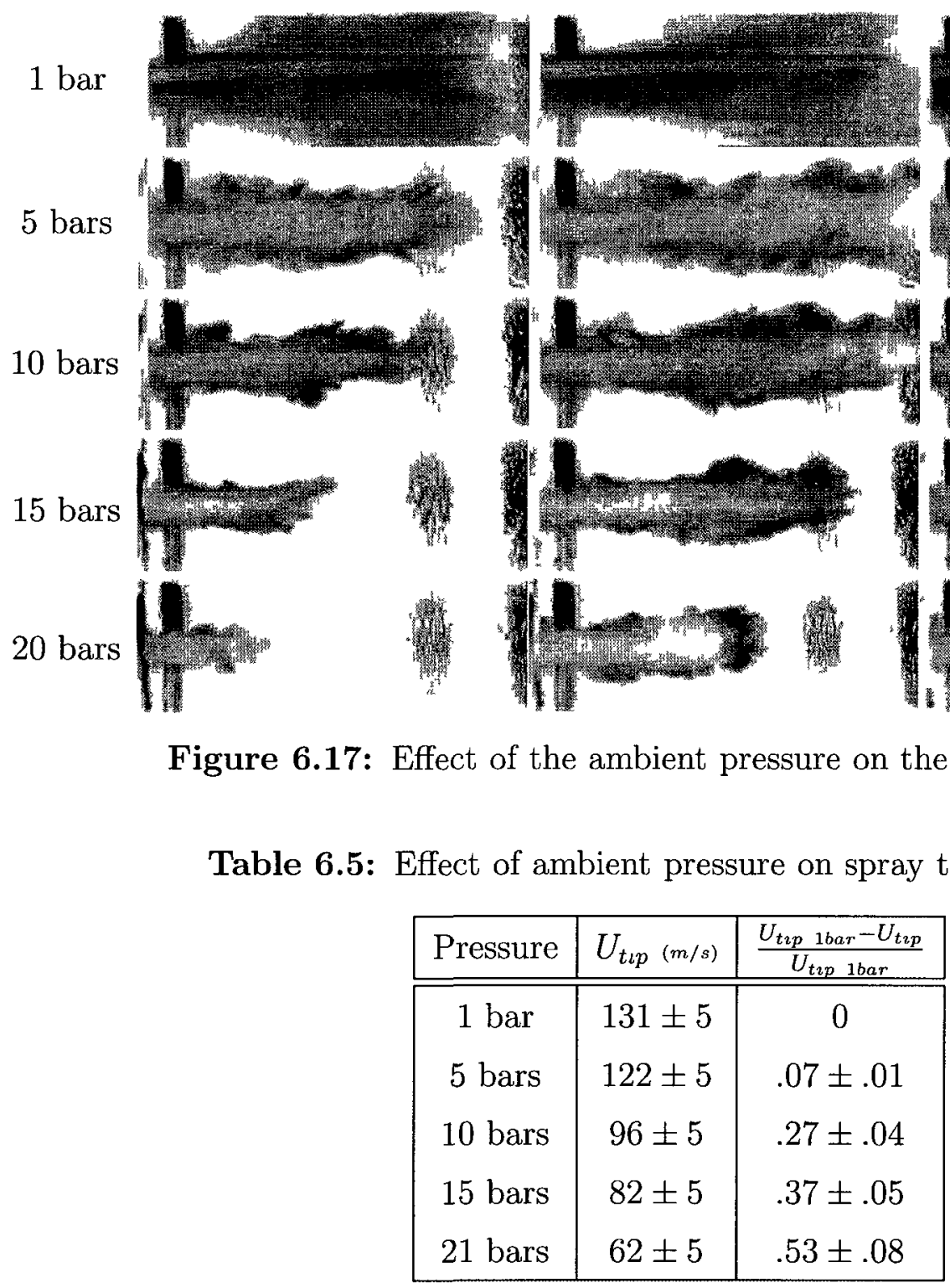




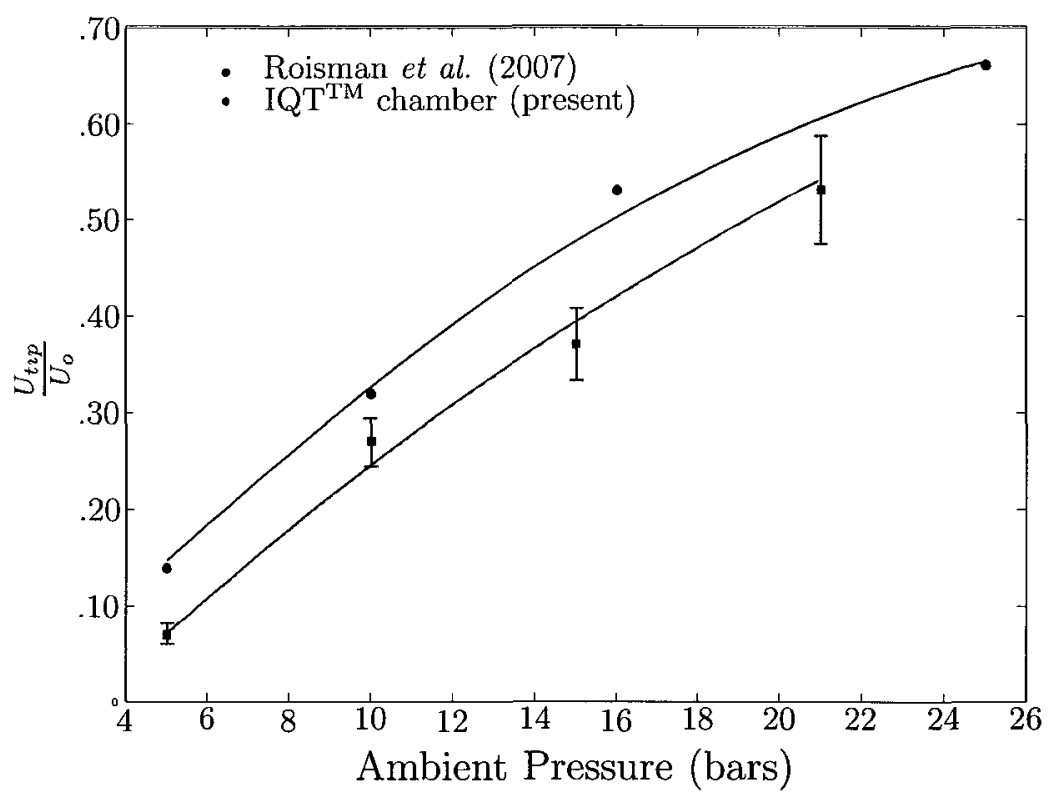

Figure 6.18: Effect of ambient pressure on spray tip velocity

The effect of ambient pressure on diesel spray penetration was previously studied by Roisman et al. [60]. Figure 6.18 demonstrates the spray tip velocity reduction from Roisman et al. [60] in comparison with results obtained in this visualization work. The small discrepancy between the IQT ${ }^{\mathrm{TM}}$ and literature is likely attributed to the different type of injectors used in the experiment. Roisman et al. used a valve covered orifice injector while the IQT ${ }^{\mathrm{TM}}$ utilizes a throttle pintle injector. The important fluid property to match in order to simulate IQT ${ }^{\mathrm{TM}}$ ambient conditions is the density ratio between the liquid fuel and the ambient air. The IQT ${ }^{\mathrm{TM}}$ operates at 21.1 bars with a charge air temperature of $550^{\circ} \mathrm{C}$. This corresponds to a density of:

$$
\rho=\frac{P}{R T}=\frac{21.1 \times 10^{5} \mathrm{~Pa}}{\left(287 \frac{\mathrm{J}}{\mathrm{kgK}}\right)(823 \mathrm{~K})}=8.93 \frac{\mathrm{kg}}{\mathrm{m}^{3}}
$$

Solving for the pressure term at an experimental test temperature of $20^{\circ} \mathrm{C}$ suggest that an ambient pressure of $[7.5 \text { bars }]_{T=293 K}$ will match the fluid density ratio of the $\mathrm{IQT}^{\mathrm{TM}}$. This penetration study suggests that the effect of IQT ${ }^{\mathrm{TM}}$ operating condition reduces the spray tip velocity $U_{t \imath p}$ by approximately $17 \%$. 


\subsection{Effect of ambient pressure on droplet size}

The effect of ambient pressure on the droplet size has been studied by Takeuchi et al. [61] on a throttling pintle nozzle similar to the one used in the IQT ${ }^{\mathrm{TM}}$. The SMD and the AMD were found to increase with the ambient pressure, which was increased from 1 to 40 bars. The increase in the SMD statistic from an atmospheric ambient pressure to the $\mathrm{IQT}^{\mathrm{TM}}$ 's ambient condition of 7.5 bars is roughly $40 \%$. Nakamura et al. [62] and Jasuka et al. [63] reported the same effect on twin fluid atomizers in a constant pressure combustor. All results demonstrate a similar increase in AMD and SMD. Figure 6.19 illustrates how the SMD increases with ambient pressure.

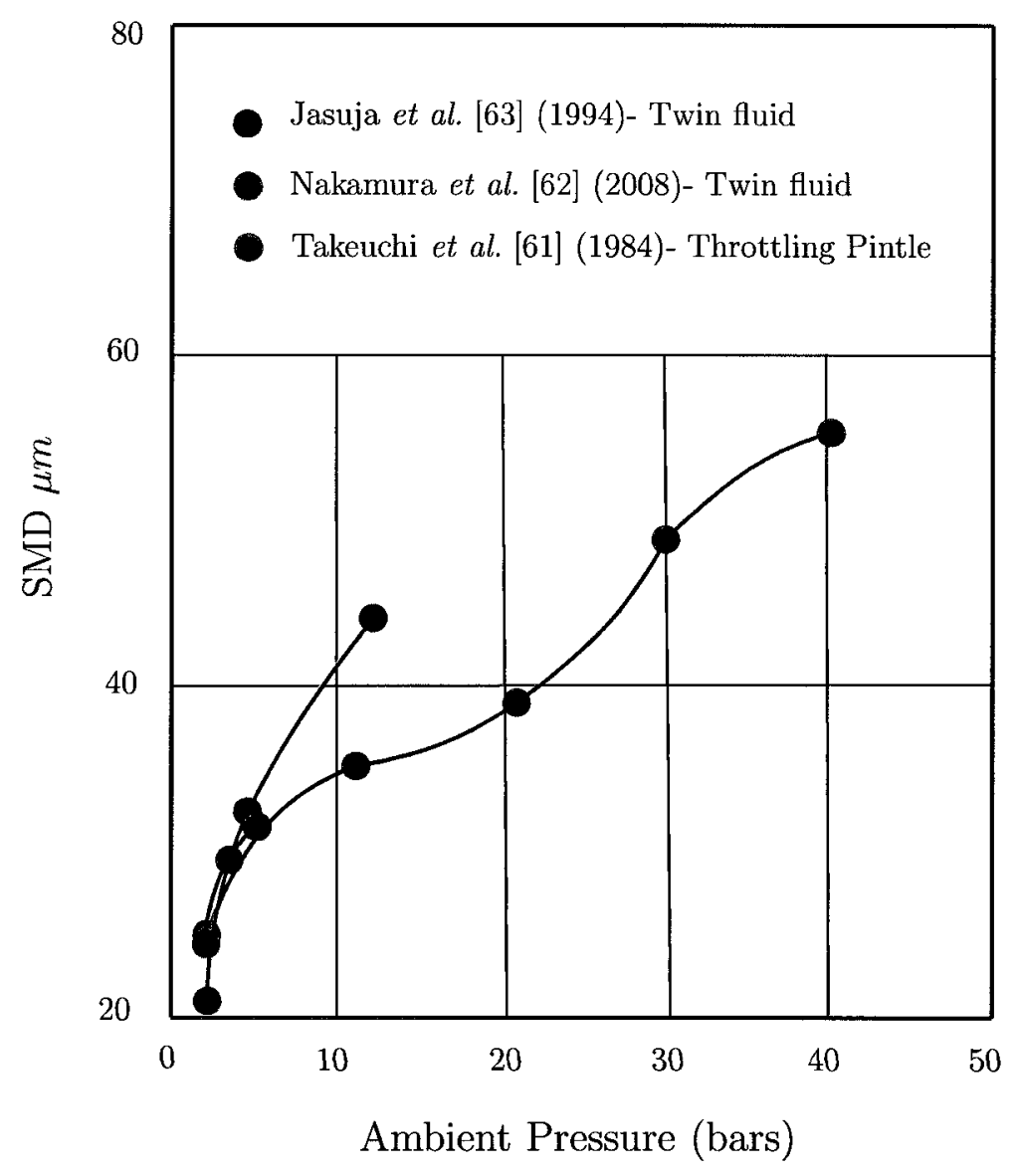

Figure 6.19: Effect of ambient pressure on SMD 
The ratio of the atmospheric ambient condition, Weber number to the IQT ${ }^{\mathrm{TM}}$ operating condition Weber number suggest better atomization as the ambient air pressure increases. The ratio of Weber numbers can be represented by Eq. (6.13) where the gas density $\rho_{g}$ is replaced with the ideal gas law $P / R T$ while assuming a similar discharged velocity at the nozzle outlet.

$$
\frac{W_{I Q T}}{W_{A t m}}=\left(\frac{P_{I Q T}}{P_{A t m}}\right)\left(\frac{T_{A t m}}{T_{I Q T}}\right)
$$

From the penetration study, this equation reduces to:

$$
\frac{W_{I Q T}}{W_{A t m}}=\left(\frac{21 b a r}{1 b a r}\right)\left(\frac{273 K}{773 K}\right)
$$

The above relation suggests a $W e_{I Q T} / W e_{\text {atm }}$ ratio of 7.42 . The larger IQT $\mathrm{TM}^{\mathrm{TM}}$ operating Weber number suggests better atomization. However, the larger droplet size reported by Takeuchi et al. [61] suggest otherwise. The main reason for this increase in droplet size is attributed to the coalescence of droplets as the ambient pressure increases. This coalescence is the result of lower droplet penetration which increases the droplet proximity. Previous work completed by Kang et al. [64] suggests that droplets with $W e<40$ tend to coalesce after impact while droplets having $W e>40$ tend to breakup. This is believed to be the main reason why droplet sizes increases with ambient pressure despite the higher Weber number [63].

\subsection{Backtracking Algorithm}

The injector discharge velocity is required in order to correlate the atmospheric ambient injection process to the IQT ${ }^{\mathrm{TM}}$ 's injection spray. The discharged velocity depends on the pressure differential $(\Delta P)$ across the injector sac and the ambient pressure. Under atmospheric ambient condition, this pressure differential is roughly 275 bars as the injection sac pressure is 276 bars compared to an ambient pressure of 1 bar. 
The $\Delta P$ under IQT ${ }^{\mathrm{TM}}$ operating condition of 21 bars is then 255 bars. The discharge velocity can be represented by Eq. (6.15) which is derived from Eq. (6.1).

$$
U=C_{\text {dis }} \sqrt{\frac{2 \Delta P}{\rho_{\text {fuel }}}}
$$

Assuming a similar discharge coefficient $C_{d i s}$ and instantaneous flow area $A$ the velocity ratio is:

$$
\frac{U_{a t m}}{U_{I Q T}}=\sqrt{\frac{\Delta P_{a t m}}{\Delta P_{I Q T}}}=1.04
$$

This relation demonstrates the similarity of the discharge velocity for the two cases. Backtracking the axial velocity to the nozzle tip under atmospheric ambient condition would give modellers a good approximation of the discharged velocity and profile for the inlet condition of the IQT ${ }^{\mathrm{TM}}$ combustion model.

The average velocity $u\left(x, r^{\prime}\right)$ at the nozzle tip $\left(x=0, r^{\prime}=0\right)$ is required in order to approximate the discharge velocity profile. The discharge velocity is found using a backtracking algorithm using results from the PDA measurements. Table 6.6 demonstrates the average velocity results on the nozzle axis.

Table 6.6: Average axial velocity

\begin{tabular}{|c|c|}
\hline Axial distance & Velocity $\mathrm{m} / \mathrm{s}$ \\
\hline \hline $35 \mathrm{~mm}$ & 86 \\
$55 \mathrm{~mm}$ & 81 \\
$70 \mathrm{~mm}$ & 74 \\
$85 \mathrm{~mm}$ & 71 \\
\hline
\end{tabular}

The differential equation governing the droplet motion is found by equating the drag force acting on the droplet to the change in droplet momentum as:

$$
\frac{C_{D} \pi d^{2}}{8} \rho_{\text {air }} u_{r e l}^{2}=\frac{\pi d^{3}}{6} \rho_{\text {liquid }} \frac{\delta u}{\delta t}
$$


This ODE simplifies to:

$$
\frac{\delta u}{\delta t}=\frac{-3 \rho_{a \iota r} C_{D} u_{r e l}^{2}}{4 \rho_{f u e l} d}
$$

This equation cannot be solved analytically as the diameter $d(k v, B)$ and drag coefficient $C_{D}(R e, B)$ are both dependent on the mass transfer Spalding number $B$ (Eq.(2.7) and Eq. (6.10)). The drag coefficient is also function of the Reynolds number $R e$ which is function of the droplet diameter $d$. The drag coefficient of evaporating droplets is reduced as the mass flux from the droplet surface fills the wake behind the droplet which lowers the pressure drag. [65]. For Reynolds number below 1000, the drag coefficient can be approximated as [66]:

$$
C_{D o}=\frac{24}{R e}\left(1+.15\left(R e^{687}\right)\right)
$$

Taking into account the reduction of drag due to droplet evaporation results in the following equation:

$$
C_{D}=\frac{C_{D o}}{1+B}
$$

The above equations are the foundation of the backtracking algorithm. The Spalding transfer number is varied between $1<B<1000$ while the initial discharge velocity is evaluated between $78 \mathrm{~m} / \mathrm{s}<U<200 \mathrm{~m} / \mathrm{s}$. The droplet diameter found using this model at $x=85 \mathrm{~mm}$ is used to validate the initial droplet diameter selection. The algorithm steps are:

- Input the initial discharge diameter of the droplet and the axial velocity values $u(35,0), u(55,0), u(70,0)$, and $u(85,0)$ into the Matlabß M-file.

- The droplet discharge velocity is then looped between $u(35,0)$ and $200 \mathrm{~m} / \mathrm{s} .($ Loop 1$)$

- The Spalding transfer number is also looped between 1 and 1000. (Loop 2)

- The droplet evaporation constant $K_{V}(B)$ is found knowing the Spalding transfer number and the liquid fuel properties using Eq. (2.6) 
- The droplet deceleration is found using a second order finite difference approximation of the differential equation Eq. (6.18) where $u(0,0)$ is the discharge velocity. The drag coefficient Eq. (6.20) used in this scheme depends strongly of the Spalding number $B$. This demonstrates the importance of looping through various Spalding numbers.

- The droplet diameter is also reevaluated after each time step knowing the droplet evaporative constant $K_{V}(B)$ using Eq. (2.7).

- The droplet velocity difference between the second order scheme and PDA results is evaluated for each locations $(x=35 \mathrm{~mm}, x=55 \mathrm{~mm}, x=70 \mathrm{~mm}, x=$ $85 \mathrm{~mm})$ and recorded for each combinations of $u(0,0)$ and $B$.

- The best result is attributed to the $u(0,0)$ and $B$ combination resulting in the smallest SSE. The result of the Matlab® M-file outputs the SSE, the Spalding number $B$, the discharge velocity $u(0,0)$ and the resulting diameter $85 \mathrm{~mm}$ downstream $d(85,0)$.

The best solution found is:

$$
\begin{aligned}
B & =37 \\
u(0,0) & =93 \mathrm{~m} / \mathrm{s} \\
d(0,0) & =17.5 \mu \mathrm{m} \\
d(85,0) & =12.7 \mu \mathrm{m}
\end{aligned}
$$

The best result is found using a discharge droplet diameter of $17.5 \mu \mathrm{m}$. The SSE of this result is surprisingly small at 0.0363 . The droplet diameter $85 \mathrm{~mm}$ downstream is also similar to the PDA diameter measurement at this axial distance which was $\mathrm{AMD} \simeq 12.9 \mu \mathrm{m}$. 
The effect of the Spalding number on the droplet penetration is illustrated in Figure 6.20(a). Large Spalding values shorten the droplet lifetime while reducing the drag coefficient considerably resulting in smaller droplet deceleration rates. Using $B=500$, complete droplet evaporation occurs at $x=65 \mathrm{~mm}$, while the velocity reduction is almost negligible. On the other hand, using $B=5$, the drag coefficient significantly reduces the droplet velocity, while retaining long droplet lifetime as the evaporation constant $K_{V}(B)$ is relatively low. This results in a stagnant droplet located at $x=$ $79 \mathrm{~mm}$ which has not yet been fully evaporated $d(79,0)=13 \mu \mathrm{m}$.
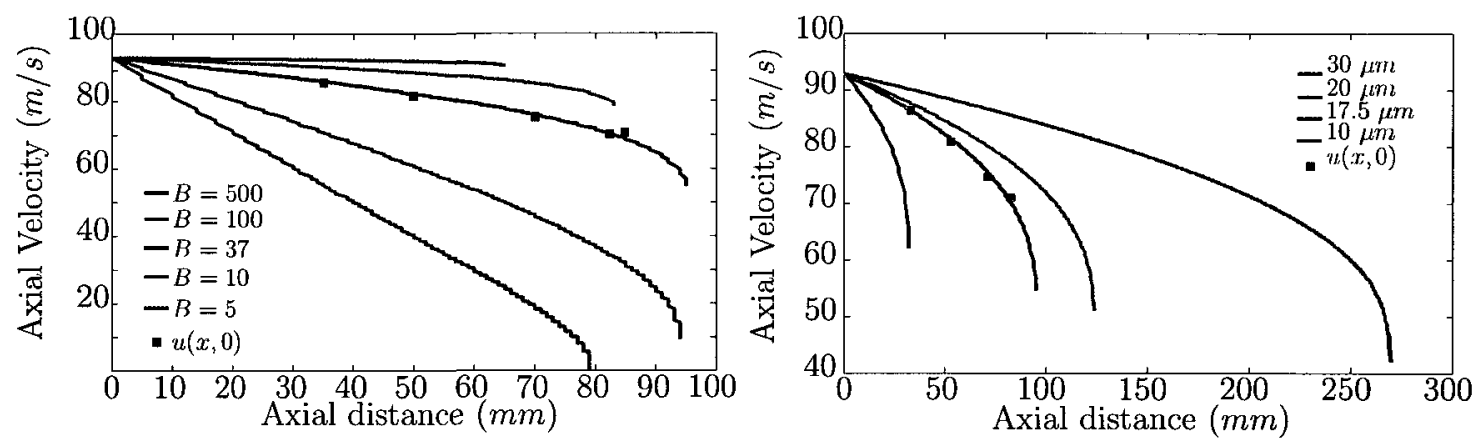

(a) Effect of the Spalding number on droplet (b) Effect of the discharge droplet diameter on penetration: Constant discharge velocity and penetration distance: Constant discharge velocdiameter ity and Spalding number

Figure 6.20: Effect of the Spalding number on spray penetration

\subsection{Discharged velocity approximation}

The IQT ${ }^{\mathrm{TM}}$ 's fuel discharge rate and the pintle nozzle hydraulic diameter $\left(D_{H}\right)$ can be approximated based on the ISO 4010 pintle injector flow characteristic standard using a inviscid incompressible flow theory [67]. The pintle injector used in the $\mathrm{IQT}^{\mathrm{TM}}$ produces a maximum flow of $4 \mathrm{~L} / \mathrm{min}$ of air at choking conditions. Knowing the ambient temperature of the ISO 4010 calibration test corresponds to a velocity of:

$$
\sqrt{\gamma R T}=343 \mathrm{~m} / \mathrm{s}
$$


The instantaneous flow area of the nozzle can be found using the volumetric flow rate expression as:

$$
A=\frac{Q}{V}=1.91 \times 10^{-7} \mathrm{~m}^{2}
$$

The fuel injected from the pintle injector forms an annular section. Knowing the pintle tip dimensions and the total instantaneous flow area, the hydraulic diameter is found to be $126 \mu \mathrm{m}$ using Eq. (6.24), where $A$ represents the wet area and $P$ represents the wet perimeter.

$$
D_{h}=\frac{4 A}{P} \rightarrow \frac{4 A}{\left(\pi D_{\imath}+\pi \sqrt{\frac{4 A}{\pi}+D_{\imath}^{2}}\right)}=126 \mu \mathrm{m}
$$

The mass of fuel injected throughout one injection cycle is roughly $0.098 \mathrm{~g}$. The total injection time is less than $4 \mathrm{~ms}$ which results in an average fuel discharge rate of 24 $\mathrm{g} / \mathrm{s}$. Converting the mass discharge rate to a volumetric rate using the fuel density results in a bulk flow rate of $Q=3.62 \times 10^{-5} \mathrm{~m}^{3} / \mathrm{s}$. The bulk fuel discharge velocity can then be approximated as:

$$
V=\frac{Q}{A}=168 \mathrm{~m} / \mathrm{s}
$$

This results in a liquid Reynolds numbers of $R e_{l}=8270$ at the nozzle outlet and a liquid Weber number of $W e_{l}=120 \times 10^{3}$ resulting in a Ohnesorge number of $Z=7.6 \times 10^{-4}$. This result suggests that the critical gas Weber number at the onset of droplet breakup is $6<W e_{g}<12$. The breakup theory presented in Section 2.2 .2 suggests that catastrophic breakup is expected at the outlet of the IQT ${ }^{\mathrm{TM}}$ injection nozzle.

Comparing the discharged velocity to the velocity found using the backtracking algorythm would suggest a velocity discharge coefficient of 0.55 , which is represented in the following equation. 


$$
C_{v}=\frac{u_{o}}{u_{t h}}=\frac{93}{168} \rightarrow 0.55
$$

Where $u_{t h}$ is the theoretical discharged velocity found from Bernoulli's principle and $u_{o}$ represents the discharged velocity from the backtracking algorithm. This value is similar to previous work performed by Nurick et al. [68] where a discharged coefficient of 0.62 was reported for a valve covered orifice nozzle.

\subsection{IQT $\mathrm{T}^{\mathrm{TM}}$ injection rate}

The injection rate of the IQT $\mathrm{TM}^{\mathrm{TM}}$ is found using the flow characteristics of the pintle style injector. All nozzles used within the IQT ${ }^{\mathrm{TM}}$ are certified under the ISO 4010 standard [67]. The ISO 4010 standard is a test method that certifies delay-type pintle nozzles for use in diesel engines. The test method is used to determine the clearance at the pintle of the nozzle. It involves pulling ambient air through the nozzle using a vacuum pump with a pressure of $0.06 \mathrm{MPa}$ below atmosphere. The pressure differential across the nozzle is sufficient to choke the flow in the effective cross section at the outlet of the nozzle. The nozzle's needle valve is lifted in small increments and the flow rate is measured at each position using a mass flow meter. The results from this test are used to generate a diagram displaying the volume flow rate through the nozzle throat as a function of the needle lift which is illustrated in Figure 6.21(a). Correlating the needle lift trace from Figure 6.1 to the injection rate is accomplished using Eq. (6.27) and Eq. (6.28) knowing the chocked velocity of air through the nozzle.

$$
A=\frac{Q}{\sqrt{\gamma R T}}
$$




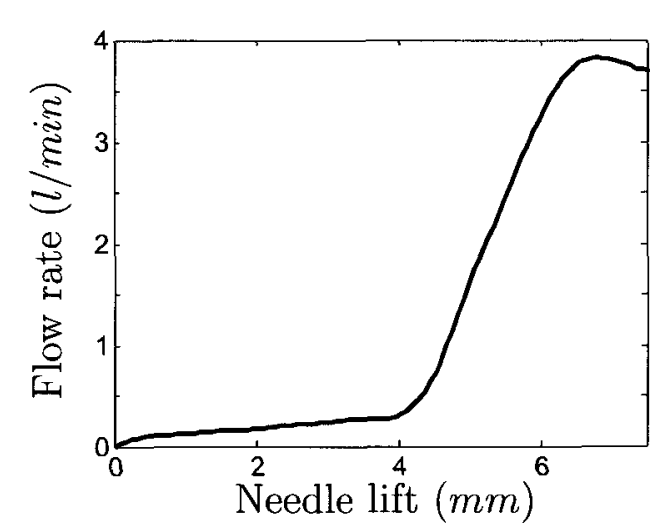

(a) Air flow rate through pintle nozzle as a function of the needle lift [67]

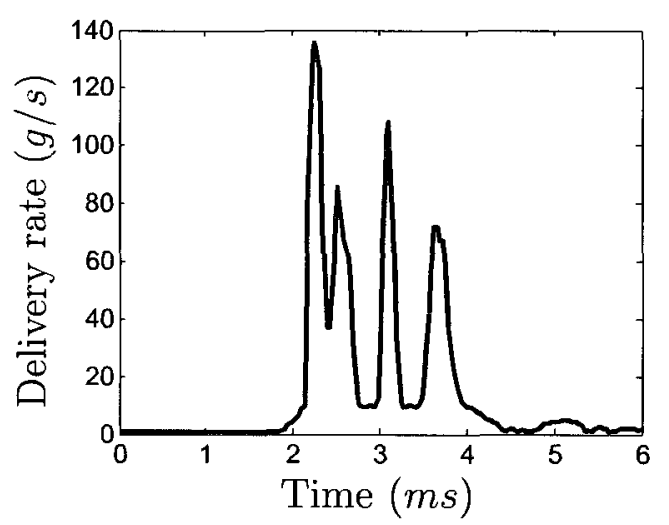

(b) Derived instantaneous mass delivery rate. $C_{D}=3.41$

Figure 6.21: $\mathrm{IQT}^{\mathrm{TM}}$ injection rate

The results of the time resolved instantaneous flow area at the nozzle outlet is then used in Eq. (6.28) to determine the injection rate.

$$
\dot{m}(t)=C_{D} Q(t) \sqrt{\frac{2 \rho_{\text {fuel }}\left(P_{\text {fuel }}-P_{\text {ambient }}\right)}{\gamma R T}}
$$

The injection rate is determined assuming a steady state discharge coefficient. The IQT $^{\mathrm{TM}}$ fuel line pressure and Viscor density are 276 bars and $0.862 \mathrm{~g} / \mathrm{ml}$ respectively. Integrating Eq. (6.28) and equating the total mass injected to $0.098 \mathrm{~g}$ (confirmed by a mass calibration of the nozzle) results in a discharge coefficient of 3.41. The resulting time resolved injection rate is illustrated in Figure 6.21(b). The injection rate profile demonstrates the impact of the nozzle needle valve fluctuations on mass delivery.

\subsection{Correlated Characteristic Equations}

The following spray characteristics correlation equations are found by fitting a Gaussian regression through the nondimensional PDA measurements. These equations can then be used as the inlet condition of combustion models by dimensionizing using the 
backtracking algorithm results presented in Section 6.7 and matching the instantaneous mass delivery rate from Section 6.9. The three main equations necessary for modelling the IQT ${ }^{\mathrm{TM}}$ are the discharge axial velocity, AMD and droplet count as a function of the inclination angle from the nozzle axis as:

$$
\begin{gathered}
u\left(0, r^{\prime}\right)=u(0,0) \exp \left(-\left[\frac{\left(r^{\prime}-\left(3 \times 10^{-13}\right)\right.}{.13}\right]^{2}\right) \\
d\left(0, r^{\prime}\right)=d(0,0)\left[.423 \exp \left(-\left[\frac{\left(r^{\prime}-\left(7.1 \times 10^{-5}\right)\right.}{.081}\right]^{2}\right)+.602 \exp \left(-\left[\frac{\left(r^{\prime}-24.9\right.}{117.1}\right]^{2}\right)\right] \\
n\left(0, r^{\prime}\right)=.665\left[\exp \left(-\left[\frac{\left(r^{\prime}-109\right.}{029}\right]^{2}\right)+\exp \left(-\left[\frac{\left(r^{\prime}+109\right.}{029}\right]^{2}\right)\right] \\
+.435\left[\exp \left(-\left[\frac{\left(r^{\prime}-167\right.}{118}\right]^{2}\right)+\exp \left(-\left[\frac{r^{\prime}+167}{118}\right]^{2}\right)\right]
\end{gathered}
$$

Figure 6.22(a) demonstrates the comparison between the PDA data and the equation in nondimensional form.
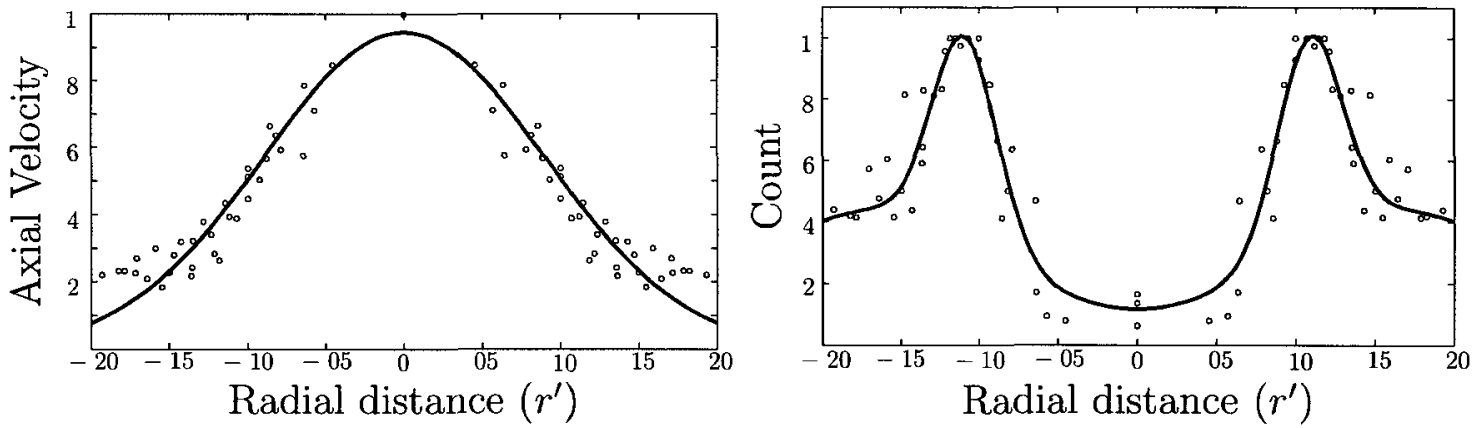

(a) Axial velocity profile correlation equation (b) Droplet count profile correlation equation compared with PDA measurement compared with PDA measurement

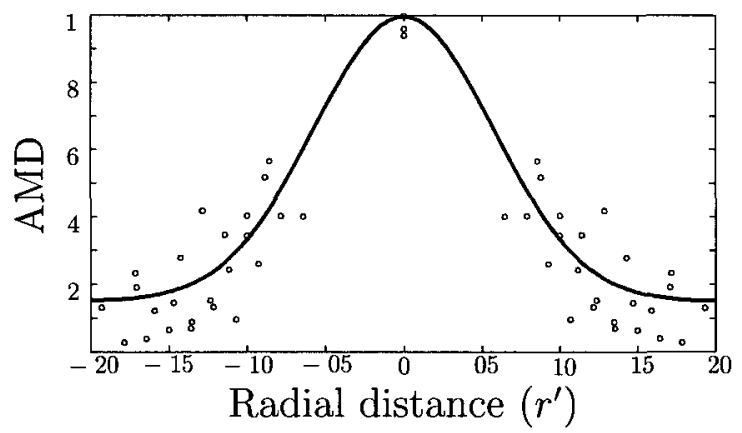

(c) Droplet diameter profile correlation equation compared with PDA measurement

Figure 6.22: Correlated spray characteristic equations 


\section{Chapter 7}

\section{Conclusions}

The IQT ${ }^{\mathrm{TM}}$ 's fuel spray was successfully characterized using PDA and front light photography.

\section{$7.1 \quad$ Summary}

\subsubsection{Qualitative Characteristics}

The initial spray is concentrated to the nozzle axis forming a poorly atomized jet. This initial jet occurs in the first $0.2 \mathrm{~ms}$ of the injection. The hollow cone quickly develops following the initial jet and is generated for approximately $3 \mathrm{~ms}$. The initial hollow cone angle was found to be $28.6^{\circ}$ which is more than twice the fully developed angle of $13^{\circ}$ found using front light photography.

\subsubsection{Quantitative Characteristics}

The droplet count suggests that most droplets form a full cone angle of $12.6^{\circ}$ which is relatively similar to the value found using front light photography. These droplets suggest an AMD of $8.0 \mu \mathrm{m}$ and a SMD of $25.0 \mu \mathrm{m}$. The axial velocity of droplets at the maximum count location was found to be $0.4 \cdot u(x)$ where $u(x)$ at the nozzle outlet was 
found to be $93 \mathrm{~m} / \mathrm{s}$ from the backtracking algorithm. Droplets located on the spray axis only accounted for $20 \%$ of the maximum count located at $12.6^{\circ}$. These droplets originate from the initial jet after the SOI and suggest an AMD of $14 \mu \mathrm{m}$, which is significantly larger than droplets located at the maximum count location. A time resolved analysis suggest a large initial hollow cone angle as the spray develops similar to results found using the CCD camera. The velocity fluctuations after the hollow cone spray, which has fully developed, are attributed to the highly transient nature of the injector pintle movement. The droplet statistics of all positions combined demonstrates an AMD of $9.3 \mu \mathrm{m}$ and an SMD of $29.3 \mu \mathrm{m}$.

\section{Effect of Mass Injected}

The amount of mass injected has a direct impact on the spray characteristics. It was found that lowering the injected mass by $16 \%$ increased the magnitude of the axial velocity throughout all radial positions. The increase in injection energy density, as mass is reduced, is responsible for this axial velocity increase. The effect of reducing mass was also found to decrease the droplet AMD by $21 \%$ while increasing the SMD statistic by $7 \%$. This result explains the increase of the dispersity parameter of the spray compared to the standard mass injected. The cause of this increase can be attributed to the change in the nozzle needle trace. As the mass injected is reduced, the local minima of the needle valve oscillations decreases leading to a lower pressure difference across the injector which causes larger and more polydisperse droplet statistics.

\section{Effect of Injection Pump Accumulator Pressure}

The injection pressure was also found to have a direct impact on spray characteristics. The largest impact was observed on the nozzle needle trace. As the injection pressure increases, the needle trace appears to be steadier during the fuel delivery. The local 
minima oscillations are almost completely eliminated. This results in similar AMD values, however the SMD of droplets is significantly reduced. Given that large droplets have greater contributions to the SMD, a reduction of the SMD suggest that the occurrence of these large droplets is reduced. Large droplets are expected to be caused by a lower pressure difference across the injector. Therefore, the needle trace supports this finding as the local minima is reduced when the injection energy increase.

\subsection{Recommendations}

The atomization characteristics were found to be quite dependent on the nozzle needle valve trace. This results from the linear relationship between the needle position and the spring pressure against the needle. In order to reduce the IQT ${ }^{\mathrm{TM}}$ instrument to instrument bias error, all needle lift traces should be made similar. It was identified from previous studies that the needle movement is highly dependent on injection pump dynamics and nozzle flow characteristics. However, this study suggests that an increase in injection energy reduces the needle fluctuations. Increasing the injection energy would reduce the needle trace differences between different IQT ${ }^{\mathrm{TM}}$ instruments. This would lead to an improvement in the unit to unit reproducibility. The size statistics reported in this thesis is not that critical for the IQT ${ }^{\mathrm{TM}}$. The importance is the size statistic difference within the $\mathrm{IQT}^{\mathrm{TM}}$ community. The important size statistic to match is the SMD. The SMD governs the physical ignition delay of the sampled fuel. Therefore, if two different IQT ${ }^{\mathrm{TM}}$ produces different SMD statistics, the total ignition delay of the sampled fuel will be different between the two instruments.

The injection energy can be increased in two methods. The easiest solution would be to increase the pressure of the accumulator. However, this option might not be feasible as some IQT ${ }^{\mathrm{TM}}$ operators use standard shop air with a volumetric intensifier 
as the accumulator air supply. A simpler solution would be to increase the pneumatic actuator size from 3 inches to 3.5 inches. This would result in an injection energy increase similar to increasing the pressure from 175 to 250 psi investigated in this study.

\subsection{Future Work}

The IQT ${ }^{\mathrm{TM}}$ combustion process should be modelled using the correlated spray equation derived in this study. In addition, using an optical IQT ${ }^{\mathrm{TM}}$ chamber, the location of auto-ignition could be compared to the model. This would be a significant contribution to researchers developing new chemical kinetic models for novel fuels using the $\mathrm{IQT}^{\mathrm{TM}}$. 


\section{List of References}

[1] American Society for Testing and Materials. ASTM D6890-09 standard test method for determination of ignition delay and derived cetane number (DCN) of diesel fuel oils by combustion in a constant volume chamber. Annual Book of ASTM Standards, 2009.

[2] Advanced Engine Technology Ltd. IQT ${ }^{T M}$ User Manual, 2009.

[3] H. Chaves, M. Knapp, A. Kubitzek, F. Obermeier, and T. Schneider. Experimental study of cavitation in the nozzle hole of diesel injectors using transparent nozzles. Society of Automotive Engineers, SAE Technical Series 950290, 1995.

[4] S. Hossainpour and A.R. Binesh. Investigation of the fuel spray atomization in a DI heavy duty diesel engine and comparison of various spray breakup models. Journal of Fuel, 2008.

[5] Dantec Dynamics. BSA flow software installation and user's guide V.4.10, 2006.

[6] H. E. Albrecht. Laser Doppler and Phase Doppler Measurement Techniques. Springer, 2003.

[7] G. Wigley, J. Heath, G. Pitcher, and A. Whybrew. Experimental analysis of the responce of a Laser/Phase Doppler anemometer system to a partially atomized spray. Particle and particle system characterization, Vol. 18:pp. 169-178, 2001.

[8] R. Payri, L. Araneo, J. Shakal, and V. Soare. Phase Doppler measurements: system set-up optimization for characterization of a diesel nozzle. Journal of Mechanical Science Technology, vol. 22(no. 8):pp. 1620-1632, 2008.

[9] M. J. Nunney. Light and Heavy Vehicle Technology. Elsevier, 2007.

[10] A.D.B. Yates, C.L. Viljoen, and A. Swarts. Understanding the relation between cetane number and combustion bomb ignition delay measurements. Society of Automotive Engineers, 2004. 
[11] American Society for Testing and Materials. ASTM D613 standard test method for cetane number of diesel fuel oil. Annual book of ASTM Standards, 2009.

[12] L.N. Allard, N.J. Hole, G.D. Webster, T.W. Ryan, D. Ott, A. Beregszazy, C.W. Fairbridge, J.Cooley, K.Mitchell, E.K. Richardson, N.G. Elliot, and D.J. Rickeard. Diesel fuel ignition quality as determined in the ignition quality tester IQT $^{\mathrm{TM}}$ - Part II. Society of Automotive Engineers, SAE Technical Series 971636 , 1996.

[13] O.J. Metcalf, A. Swarts, and A. Yates. A study of the ignition-delay character of n-heptane in the IQT ${ }^{\mathrm{TM}}$ combustion bomb using CFD modelling. Society of Automotive Engineers, SAE Technical Series 2007-01-0021, 2007.

[14] G.E. Bogin, A.M. Dean, A. DeFilippo, J.Y. Chen abd G. Chin, J. Luecke, M.A. Ratcliff, and B.T Zigler. Modelling the fuel spray and combustion process of the ignition quality tester with KIVA-3V. Fall Meeting of the Western States Section of the Combustion Institute, NREL/CP-540-4673, 2010.

[15] N. A. Henein and A. N. Fragoulis. Correlation between physical properties and auto-ignition parameters of alternate fuels. Society of Automotive Engineers, SAE Technical Series 850266, 1985.

[16] T. J. Callahan, T. W. Ryan, L. G. Dodge, and J. A. Shwalb. Effects of fuel properties on diesel spray characteristics. Society of Automotive Engineers, 870533, 1987.

[17] M. M. Karakolis. Experimental analysis of the fuel injection system of the ignition quality tester (IQT ${ }^{\mathrm{TM}}$ ). Master's thesis, Carleton University, 2009.

[18] H.K. Suh and C.S. Lee. Effect of cavitation in nozzle orifice on the diesel fuel atomization characteristics. International Journal of Heat and Fluid Flow, vol. 29(issue 4):pp. 1001-1009, 2008.

[19] C. Bae and J. Kang. The structure of a break-up zone in the transient diesel spray of a valve-covered orifice nozzle. Internation Journal of Engine Research, vol. 7, 2006.

[20] G.J. Smallwood and Omer L. Gulder. Views on the structure of transient diesel sprays. Atomization and Sprays, vol. 10:355-386, 2000.

[21] H. Kamoun, G. Lamanna, and B. Weigand. High-speed shadowgraphy investigations of superheated liquid jet atomization. Proceedings - Annual Conference on Liquid Atomization and Spray Systems, 2010. 
[22] O.L. Gulder, G.J. Smallwood, and D.R. Snelling. Diesel spray structure investigation by laser diffraction and sheet illumination. Socrety of Automotive Engineers, SAE Subsciption 920577, 1992.

[23] T.B Low and R. List. Collision, coalescence and breakup of raindrops. Journal of Atmospherıc Scrence, vol. 39:pp. 1591-1606, 1982.

[24] L.Allocca, S.Alfuso, L.Marchitto, and G.Valentino. GDI multi-hole injector: Particle size and velocity distribution for single and jet-to-jet evolution analysis. Internatıonal Annual Conference on Lıquid Atomızatıon and Spray Systems, 2009 .

[25] M. A. Rahman, J. Gomez, M. Balzan, and B. A. Fleck. Predicting the two-phase gas/liquid spray break-up mechanism by the dimensionless numbers. ILASS Europe 2010 - Proceedings of the 23rd Annual Conference on Liquid and Spray Systems, 2010.

[26] G.M. Faeth. Structure and atomization properties of dense turbulent sprays. Symposıum on Combustion, pages pp. 1345-1352, 1990.

[27] T. Wakisaka, Y. Shimamoto, Y. Isshiki, S. Akamatsu, and K. Ibaraki. Improvement in a droplet breakup model for numerical analysis of fuel sprays. Socrety of Automotice Engineers of Japan, vol. 18(issue 1):pp. 3-10, 1996.

[28] R.C. Reid, J.M. Prausnitz, and B.E. Poling. The Propertres of Gases and Liquids. McGraw-Hill, 4th edition edition, 1987.

[29] W.L.H. Hallet. Combustıon in Diffusıon Systems. University of Ottawa, 2009.

[30] L. Zhang, T. Tsurushima, T. Ueda, Y. Ishii, T. Itou, and T. Minami. Measurement of liquid phase penetration of evaporating spray in a DI diesel engine. Socıety of Automotive Engineers, SAE Technical Paper 971645, 1997.

[31] G. Solero and A. Coghe. Effect of injection topology on turbulent homogeneous

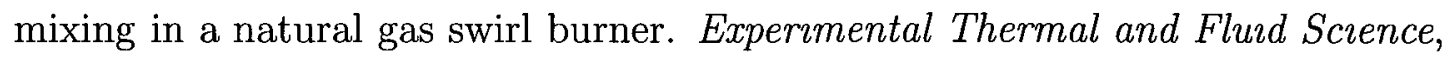
Vol. 21, Issues 1-3:pp. 162-170, 2000.

[32] S. Bernero, A. Glauser, and M. Zajadatz. Cold flow PIV and spray visualization experiments applied to the development of ALSTOM dual fuel gas turbine burners. 13th Int. Symp. on Applications of Laser Techniques to Flund Mechanıcs, 2006. 
[33] E. Kristensson, E. Berrocal, R. Wellander, M. Ritcher, M. Alden, and M. Linne. Structured illumination for 3-D Mie imaging and 2-D attenuation measurements in optically dense sprays. Proceedings of the Combustion Institute, vol. 33:pp. 855-861, 2010.

[34] J. Karnawat and A. Kushari. Controlled atomization using a twin-fluid swirl atomizer. Experiments in Fluids, Vol. 41:pp. 649-663, 2006.

[35] L. Thiery, J. Prenel, and R. Porcar. Theoretical and experimental intensity analysis of laser light sheets for flow visualization. Optics Communications, Vol. 123(issue 4-6):pp. 801-809, 1996.

[36] S. Tavoularis. Measurements in Fluid Mechanics. Cambridge University Press, 2005.

[37] S.N. Soid and Z. A. Zainal. Spray and combustion characterization for internal combustion engines using optical measuring techniques - a review. Energy, Vol. 36:pp. 724-741, 2011.

[38] S. Moon, Y. Matsumoto, K. Nishida, and J. Gao. Gas entrainment characteristics of diesel spray injected by a group-hole nozzle. Fuel, vol. 89:pp. 3287-3299, 2010.

[39] O.A. Kuti, K. Nishida, J.Y. Zhu, W. Zhang, X.G. Wang, and Z.H. Huang. Spray and flame characteristics of biodiesel fuel injected by common rail injection system for DI Diesel engine. Asia-Pacific Conference on Combustion, 2010.

[40] Dodge L. G., Rhodes D. J., and Reitz R. D. Drop-size measurement techniques for sprays: comparison of Malvern laser-diffraction and Aerometrics phase/Doppler. Applied Optics, Vol. 26(no. 11):pp. 2144-2154, 1987.

[41] F. Durst and M. Zar. Laser Doppler measurements in two-phase flows. In Proc. LDA Symp (Copenhagen), pages pp. 403-429, 1975.

[42] Naqwi A. and Ziema M. Extended phase Doppler anemometry for sizing particles smaller than 10 microns. Journal of Aerosol Science, Vol. 23:pp. 613-621, 1992.

[43] W. D. Bachalo and M. J. Houser. Phase / Doppler spray analyzer for simultaneous measurements of drop size velocity distributions. Optical Engineering, vol. 23(No. 5):pp. 583-590, 1984.

[44] H. Z. Cummins and Y. Yeh. Localized fluid flow measurements with an He-Ne laser spectrometer. Applied Physics Letters, vol. 4:pp. 176-178, 1965. 
[45] J. A. Lock and G. Gouesbet. Generalized Lorenz Mie theory and applications. Journal of Quantitative Spectroscopy and Radiative Transfer, vol. 11(issue 1):pp. 22-34, 2009.

[46] L. Araneo and C. Tropea. Improving phase Doppler measurement in a dense diesel spray. Society of Automotive Engineers, SAE Technical Series 2000-01$2047,2000$.

[47] R. Kapulla and S.B. Najera. Operation conditions of a phase doppler anemometer: Droplet size measurements with laser beam power, photomultiplier voltage, signal gain and signal-to-noise ratio as parameters. Measurement Science and Technology, Vol. 17:pp. 221-227, 2005.

[48] C. Behrouz. Preliminary drop size and velocity measurements in a dense dieseltype spray. Society of Automotive Engineers, 1990.

[49] J. Koo and J. K. Martin. Droplet sizes and velocities in a transient diesel fuel spray. Society of Automotive Engineers, SAE Technical Series 900397, 1997.

[50] D. G. Alciatore and M. B. Histand. Introduction to Mechatronics and Measurement Systems. McGraw Hill Higher Education, third edition edition, 2007.

[51] K-J Wu, D. A. Santavicca, and F. V. Bracco. LDV measurements of drop velocity in diesel-type sprays. American Institute of Aeronautics and Astronautics, Vol. 22:pp. 1263-1270, 1984.

[52] M. Yoshioka, S. Sato, and T. Kikuchi. A method for measuring the frequency responce of photo detector modules using twice modulated light. Journal of Lightwave Technology, Vol. 23(issue 6):pp. 2112-2120, 2005.

[53] American Society of Mechanical Engineers. Measurement uncertainty, instruments and apparatus, 1985.

[54] J.S. Bendat and A.G. Piersol. Random Data: Analysis and Measurement Procedures. Wiley-Interscience, 1986.

[55] T. W. Ryan. Diesel engine injection and combustion of slurries of coal, charcoal, and coke in diesel fuel. Society of Automotive Engineers, SAE Technical Series 840119, 1984.

[56] D.J. Kim and J.K. Lee. Analysis of the transient atomization characteristics of diesel spray using time-resolved PDPA data. Automotive Journal of Automotive Technology, vol. 9(no. 3), 2008. 
[57] C. Arcoumanis, J.C. Chang, and T. Morris. Spray characteristics of single and two-spring diesel fuel injectors. Society of Automotive Engineers, SAE, 1993.

[58] J.M. Nouri, M.A. Hamid, Y. Yan, and C. Arcoumanis. Spray characterization of a piezo pintle-type injector for gasoline direct injection engines. Journal of Physics, vol. 85(issue 1), 2007.

[59] Ja-Ye Koo and Jong-Hyun Kim. Assessment of a phase Doppler anemometry technique in dense droplet laden jet. KSME International Journal, Vol. 17(no. 7):pp. 1083-1094, 2003.

[60] I.V. Roisman, L. Araneo, and C. Tropea. Effect of ambient pressure on penetration of a diesel spray. International Journal of Multiphase Flow, vol. 33(issue 8):pp.904-920, 2007.

[61] K. Takeuchi, J. Senda, and K. Yamada. Droplet size distribution in diesel fuel spray (continued: Effects of back pressure and ambient density). Japan Society of Mechanical Engineers, vol. 27:pp. 2211-2218, 1984.

[62] M. Nakamura, S.M. Hwang, Y. Nakao, D. Nishioka, J. Hayashi, and F. Akamatsu. Measurement of spray characteristics of coaxial jet spray flames under high pressure. IASME/WSEAS Int. Conf. on Energy and Environment, pages pp. 51-54, 2008.

[63] A. Jasuja and A. Lefebvre. Influence of ambient pressure on drop-size and velocity distributions in dense sprays. Twenty-Fifth Symposium on Combustion/The Combustion Institute, vol. 25:pp. 345-352, 1994.

[64] S-J. Kang, E-S. Lee, K-C. Kwon, J-H. Oh, and M-J. Yu. Twin spray characteristics between two impinging f-o-o-f type injectors. Korean Society of Mechanical Engineers KSME, vol. 16(No. 5):pp. 732-742, 2002.

[65] P. Chuchottaworn, A. Fulinami, and K. Asano. Experimental study of evaporation of a volatile pendant drop under high mass flux conditions. Journal of Chemical Engineering, vol. 17:pp. 7-13, 1984.

[66] L.D. Smoot and D.T. Pratt. Pulverized Coal Combustion and Gasification. Plenum Press., 1979.

[67] International Organization for Standardization. Iso 4010: Diesel engines - calibration nozzle, delay pintle type. ISO Standard, 1998. 
[68] Nurick W.H. Orifice cavitation and its effect on spray mixing. Journal of Fluid Engineering, pages pp. 681-687, 1976. 


\section{Appendix A}

\section{Fluid Properties}

Diesel oil and Viscor fluid properties :

\begin{tabular}{|c|c|c|}
\hline Property & No. 2 Diesel Fuel ${ }^{1}$ & Viscor $\mathrm{NF}^{2}$ \\
\hline Chemical formula & $C_{3}$ to $C_{2} 5$ & NA \\
\hline Molecular weight & $\simeq 200$ & NA \\
\hline Specific gravity @ $60^{\circ} \mathrm{F}$ & $.81 \leftrightarrow .89$ & .827 \\
\hline Density $l b / g a l @ 60^{\circ} \mathrm{F}$ & $6.7 \leftrightarrow 7.4$ & 6.9 \\
\hline Boiling temperature ${ }^{\circ} \mathrm{F}$ & $370 \leftrightarrow 650$ & NA \\
\hline Cetane number & $40 \leftrightarrow 55$ & NA \\
\hline Freezing point ${ }^{o} \mathrm{~F}$ & $-40 \leftrightarrow-30$ & -17 \\
\hline Viscosity cP @ $60^{\circ} \mathrm{F}$ & $2.6 \leftrightarrow 4.1$ & 2.6 \\
\hline Latent heat of evaporation @ $60^{\circ} \mathrm{F} \frac{B T U}{l b}$ & 100 & NA \\
\hline
\end{tabular}

\footnotetext{
${ }^{1}$ Diesel Fuel Oils, 1987, Petroleum Product Surveys, National Institue for Petroleum and Energy Research, October 1987.

${ }^{1}$ Kroon-Oil B.V. maintenance products: Product information 06:30:30
} 


\section{Appendix B}

\section{Phase Doppler Anemometry}

\section{Experimental Data}

B.1 PDA results at standard mass, standard injection energy

\section{B.1.1 $x=35 \mathrm{~mm}$}

The following pages illustrates results $35 \mathrm{~mm}$ away from the nozzle tip: 

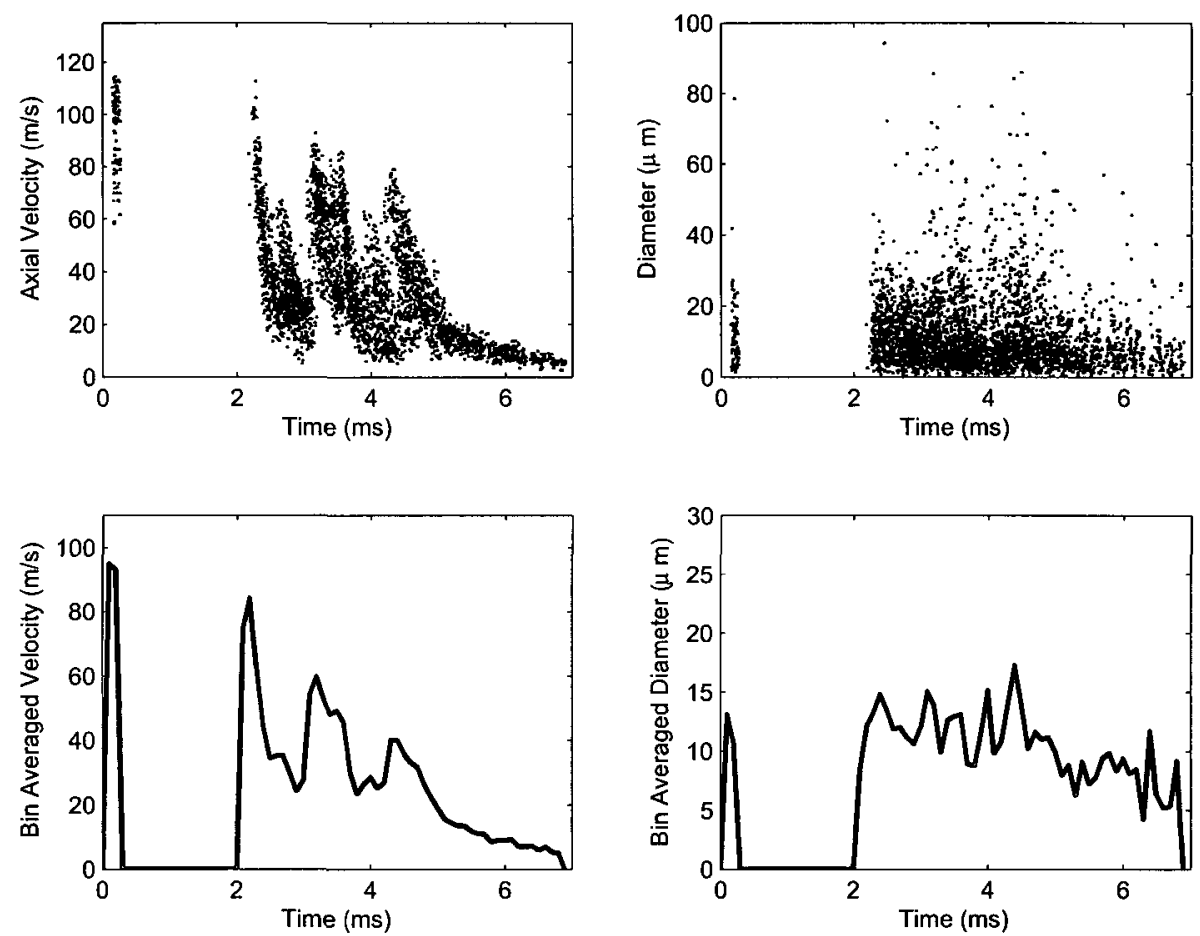

(a) Axial velocity and diameter vs time
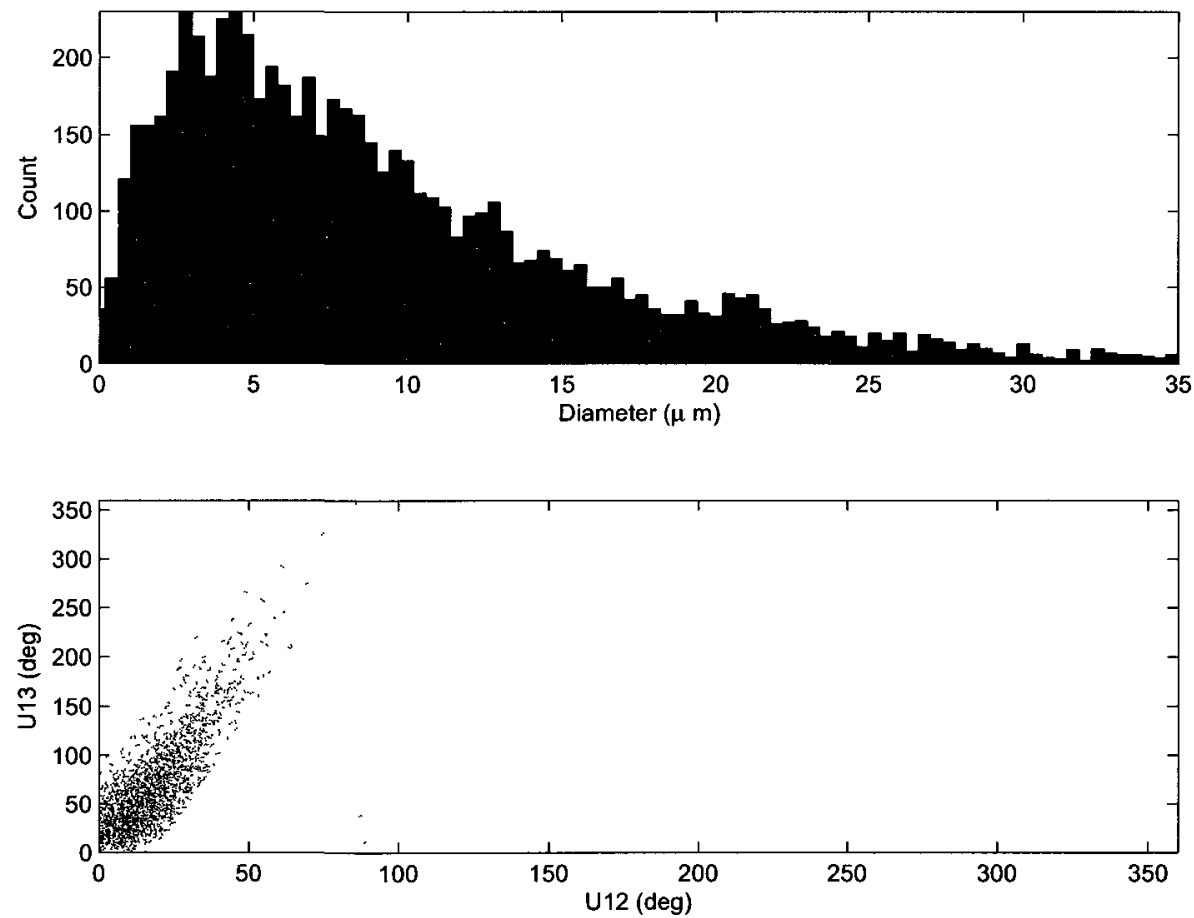

(b) Diameter histogram and PDA detector phase validation

Figure B.1: $\mathrm{x}=35 \mathrm{~mm}, \mathrm{r}=0 \mathrm{~mm}$ 

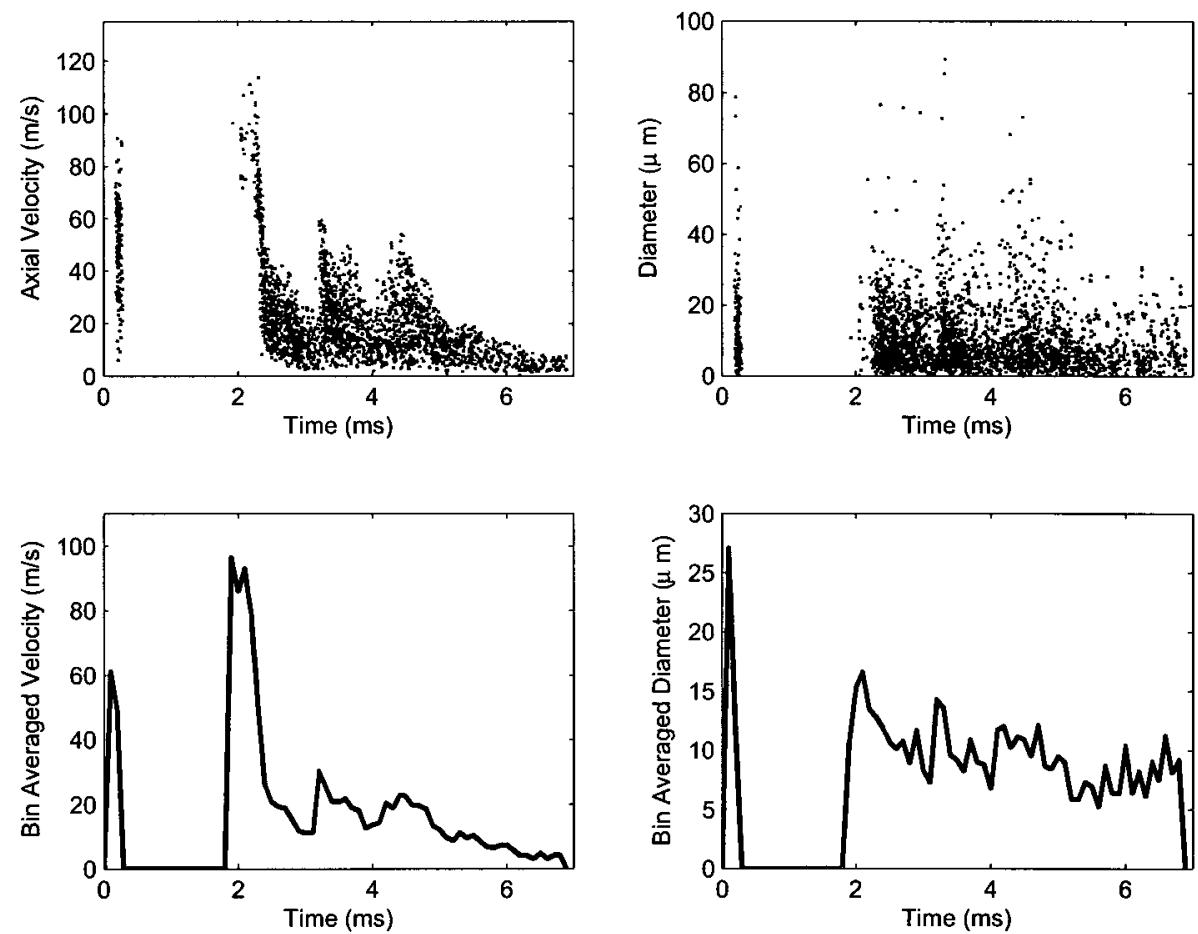

(a) Axial velocity and diameter vs time
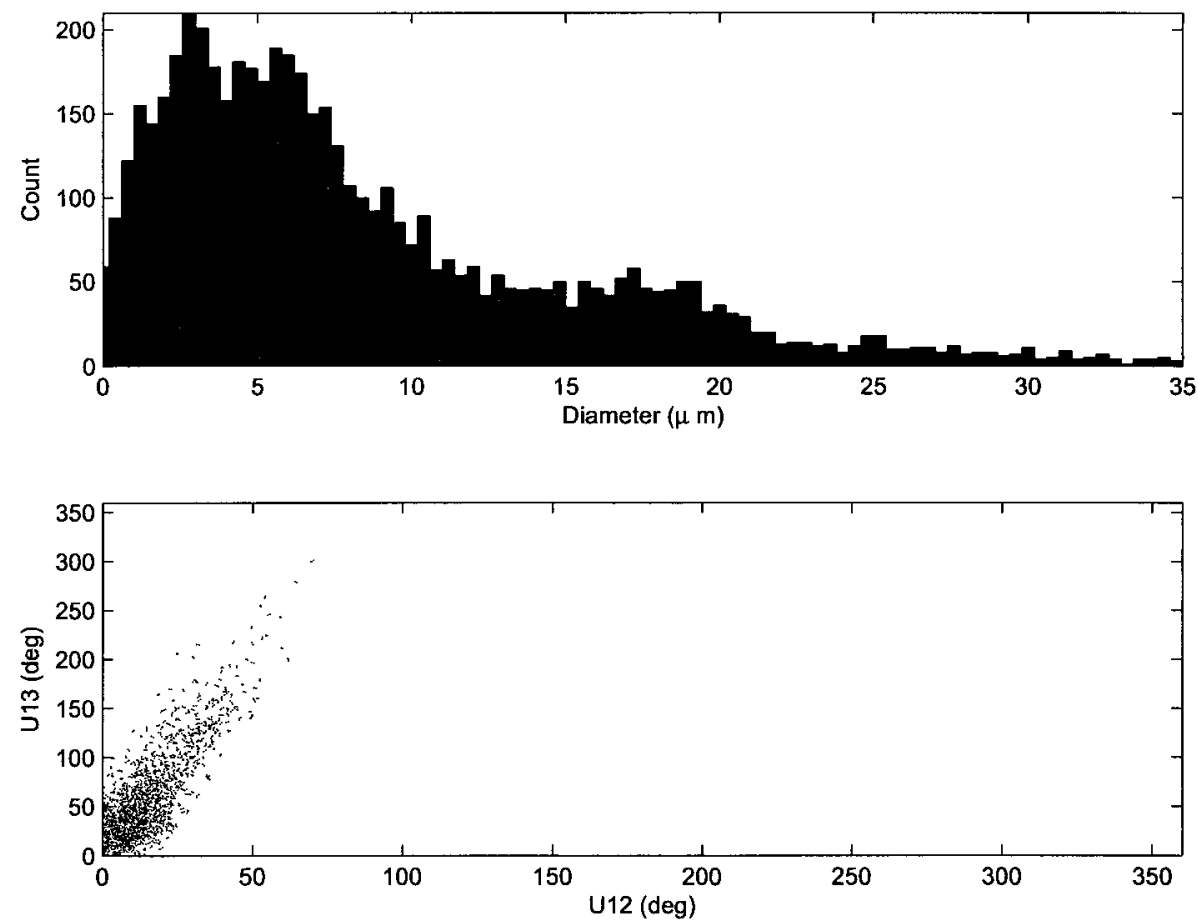

(b) Diameter histogram and PDA detector phase validation

Figure B.2: $\mathrm{x}=35 \mathrm{~mm}, \mathrm{r}=2 \mathrm{~mm}$ 

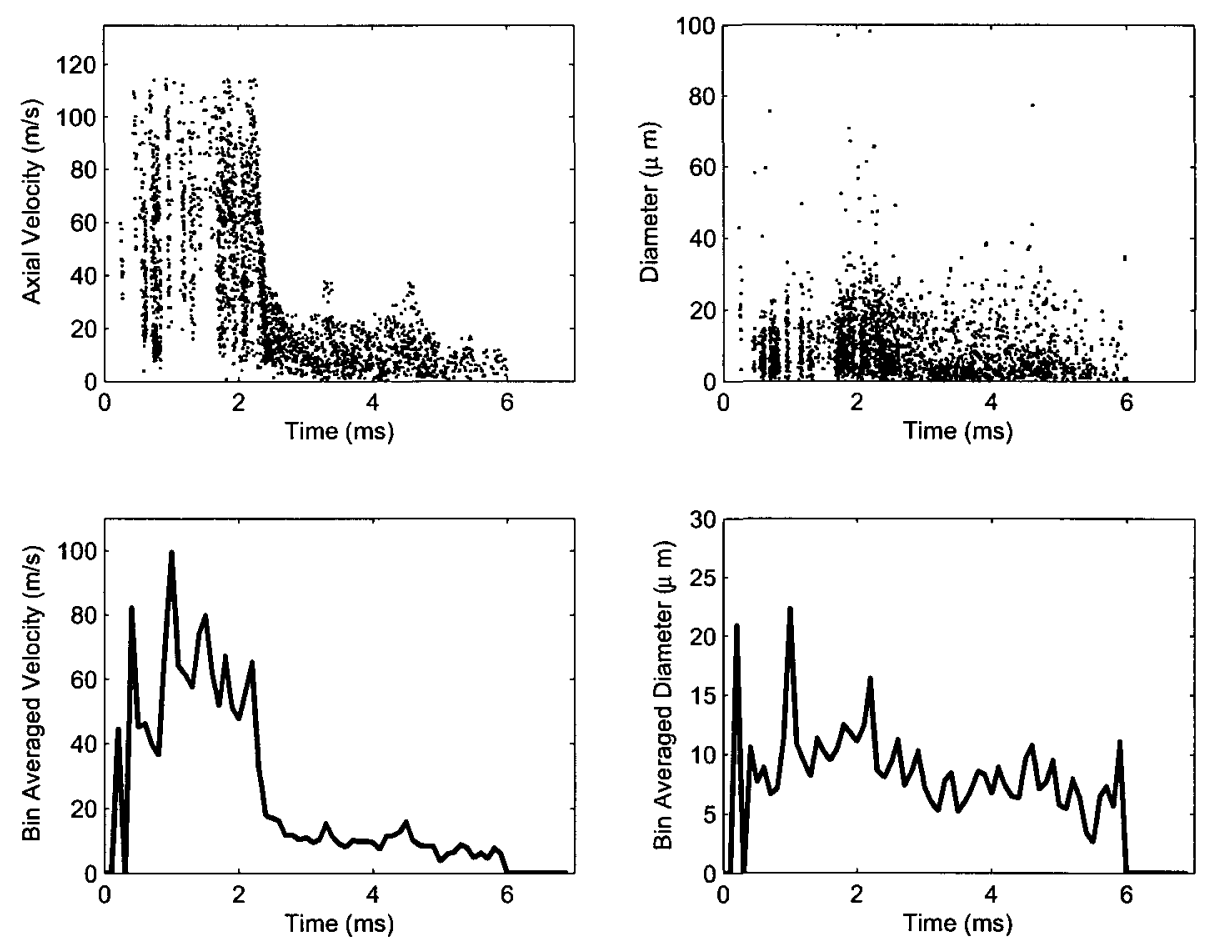

(a) Axial velocity and diameter vs time
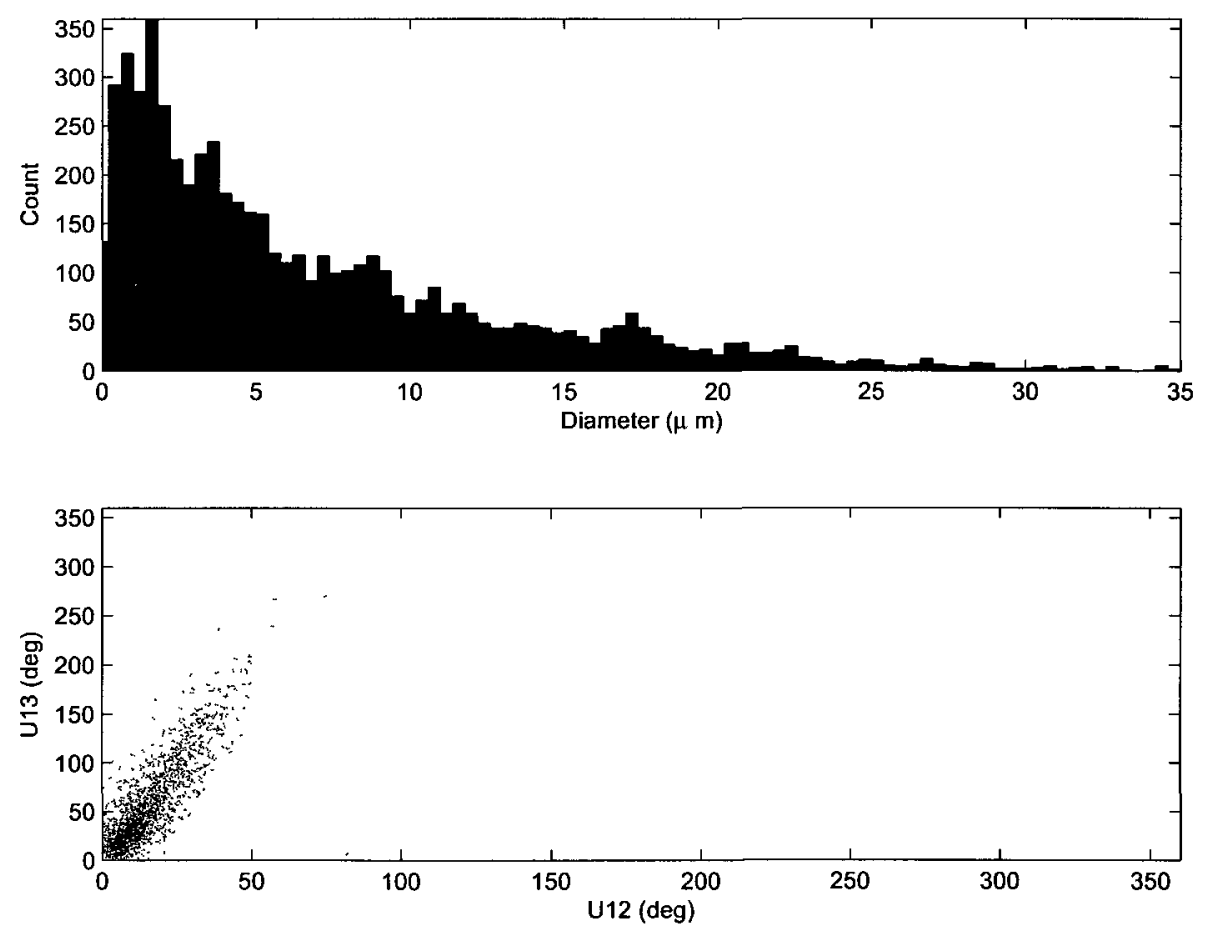

(b) Diameter histogram and PDA detector phase validation

Figure B.3: $\mathrm{x}=35 \mathrm{~mm}, \mathrm{r}=3 \mathrm{~mm}$ 

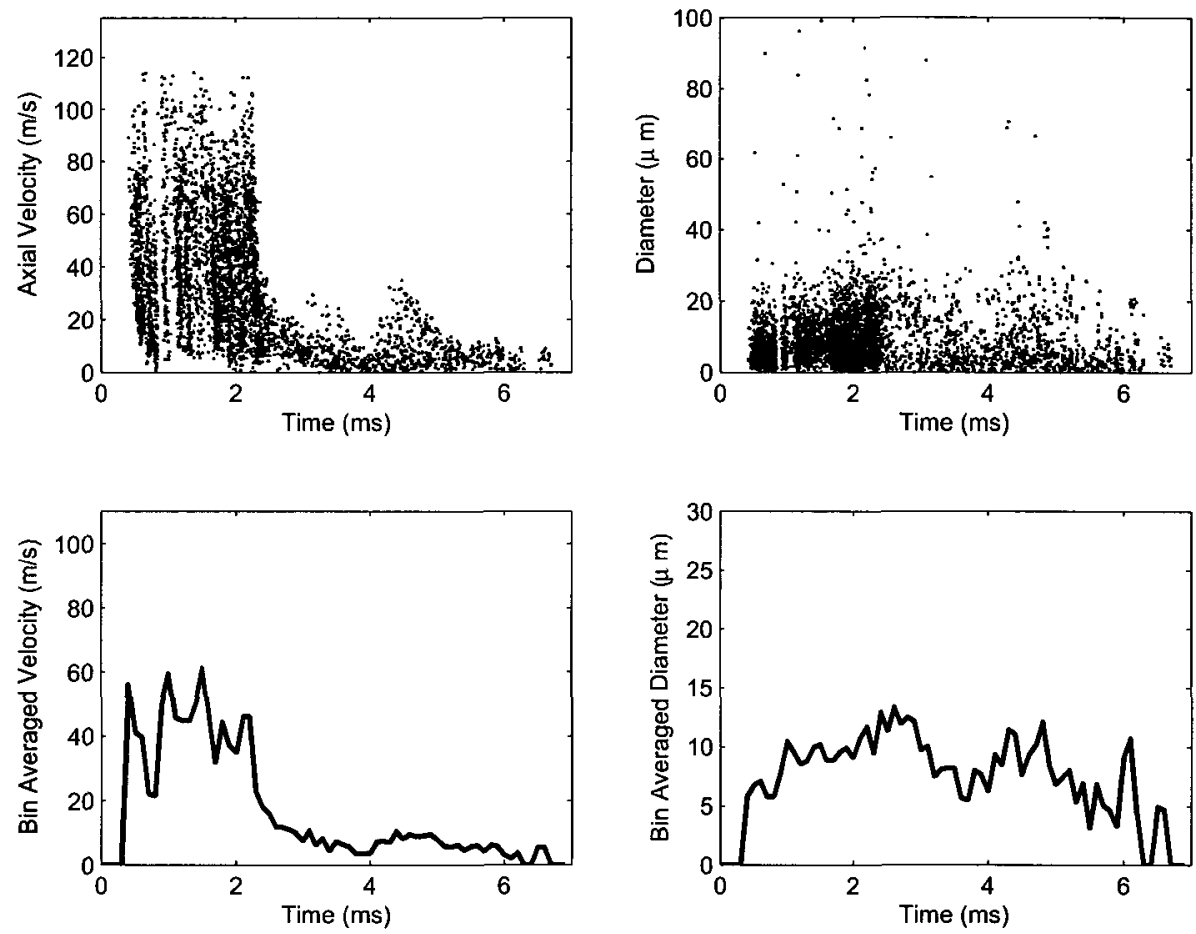

(a) Axial velocity and diameter vs time
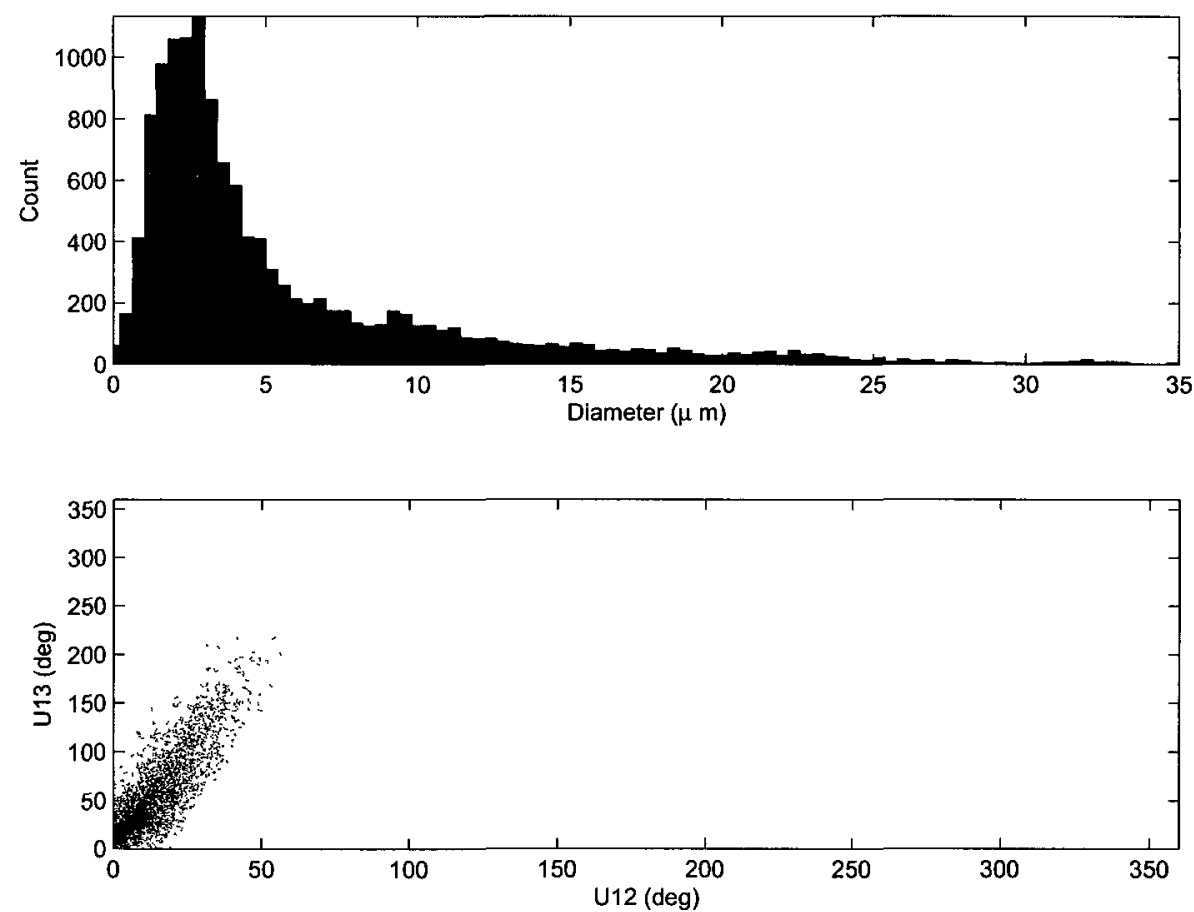

(b) Diameter histogram and PDA detector phase validation

Figure B.4: $\mathrm{x}=35 \mathrm{~mm}, \mathrm{r}=3.5 \mathrm{~mm}$ 

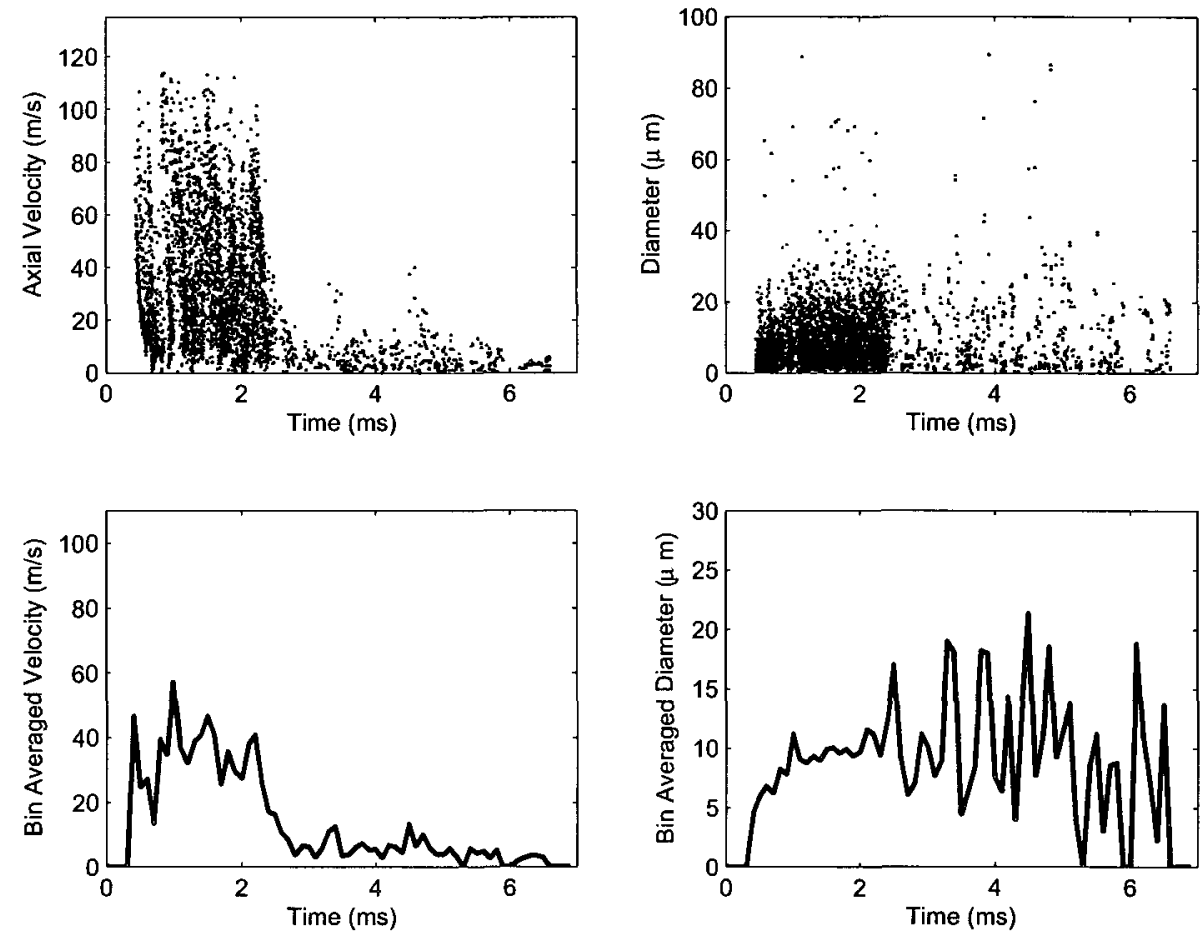

(a) Axial velocity and diameter vs time
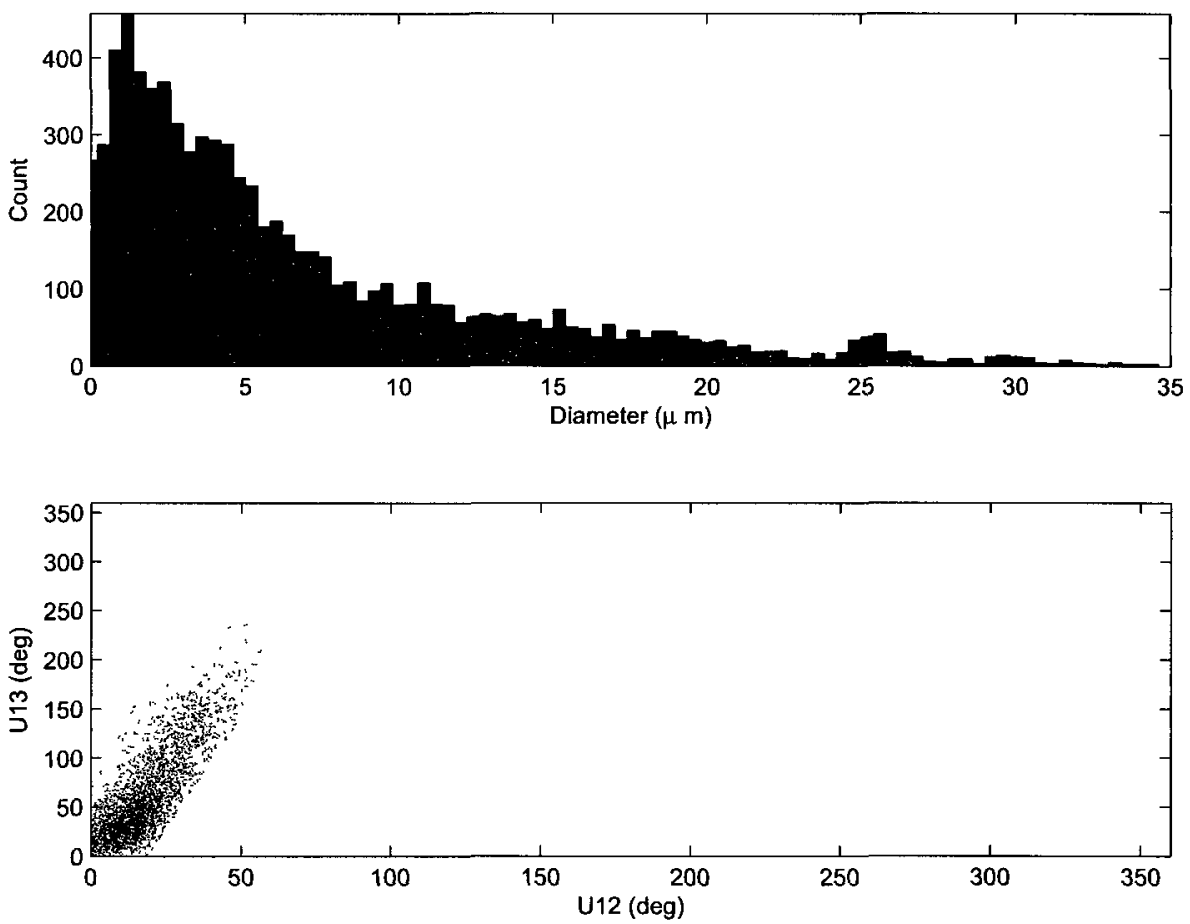

(b) Diameter histogram and PDA detector phase validation

Figure B.5: $\mathrm{x}=35 \mathrm{~mm}, \mathrm{r}=4 \mathrm{~mm}$ 

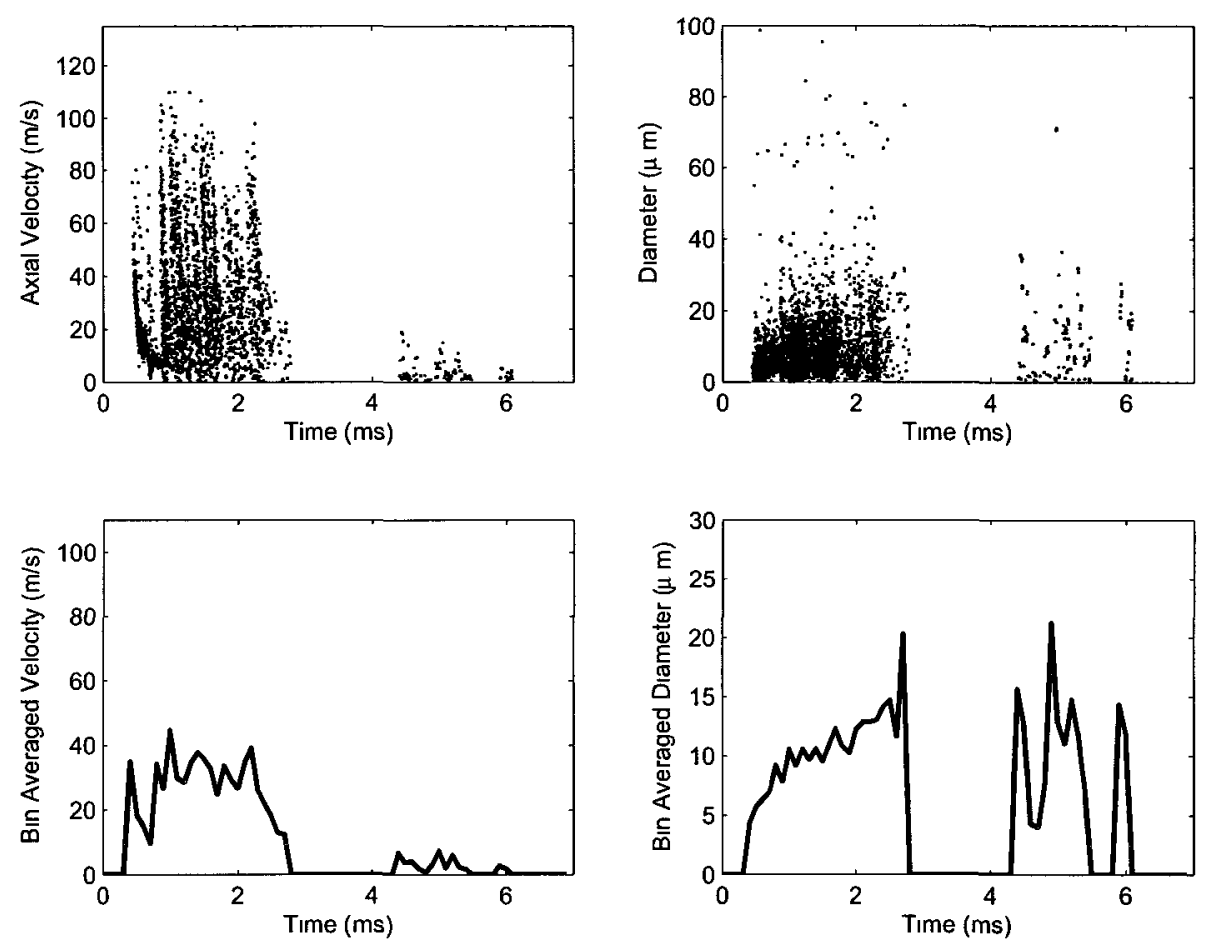

(a) Axial velocity and diameter vs time
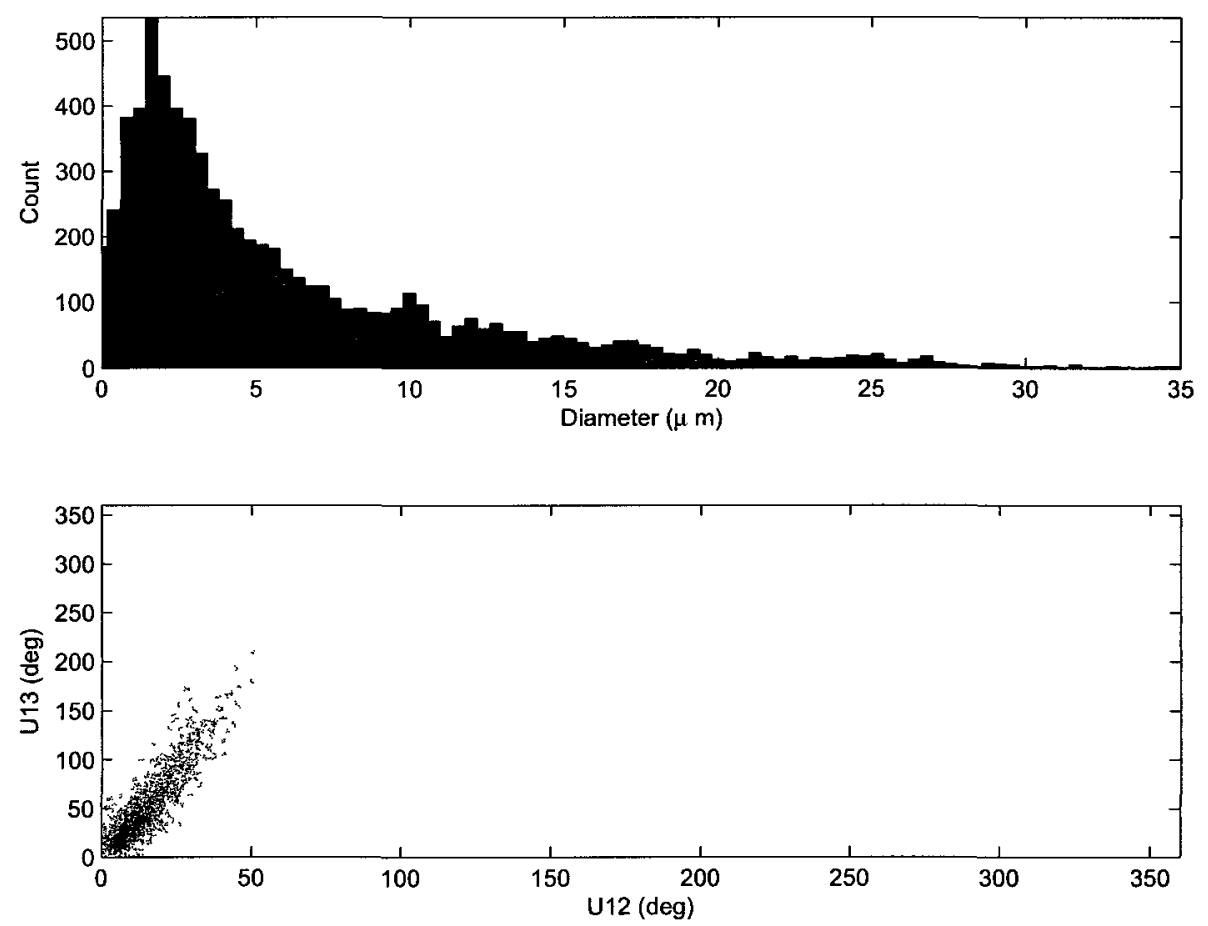

(b) Diameter histogram and PDA detector phase validation

Figure B.6: $x=35 \mathrm{~mm}, \mathrm{r}=4.5 \mathrm{~mm}$ 

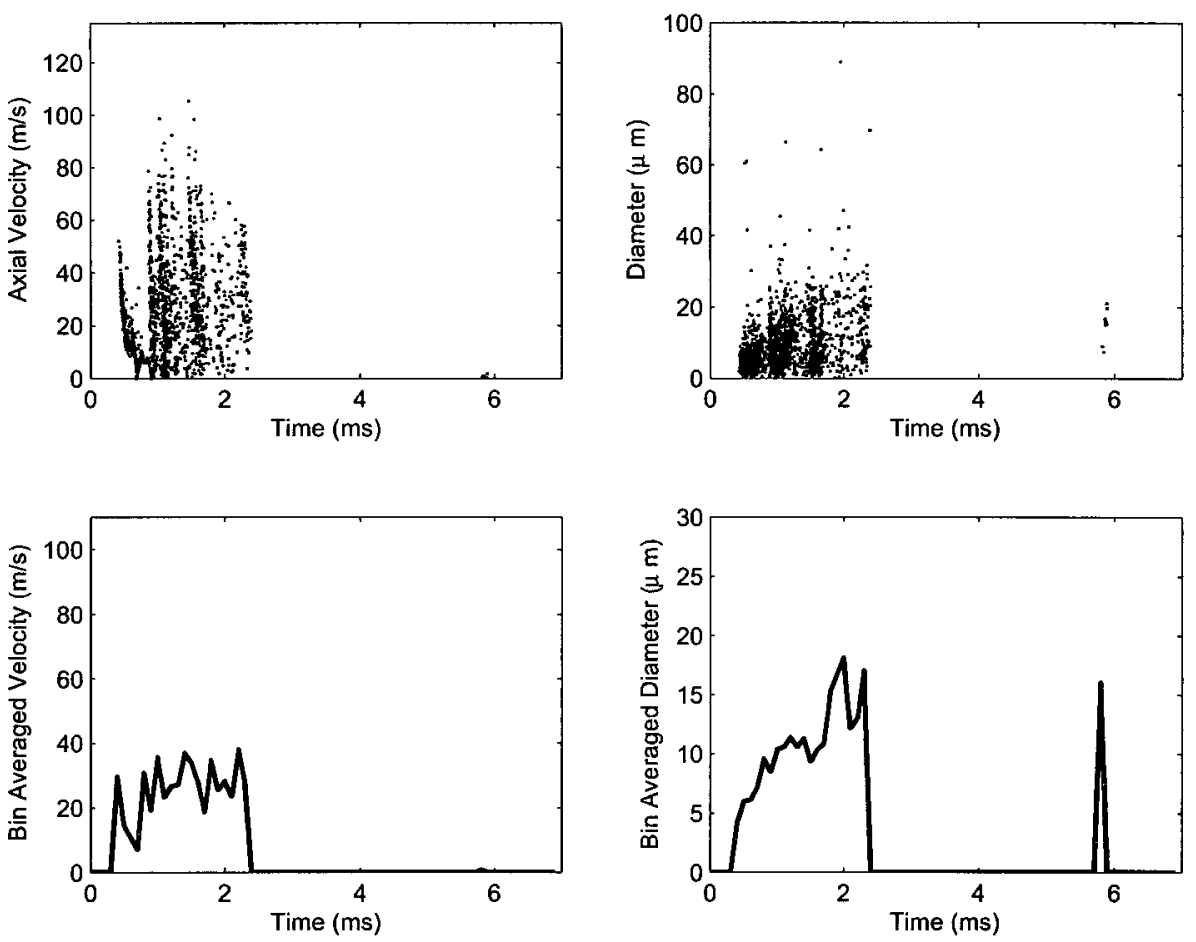

(a) Axial velocity and diameter vs time
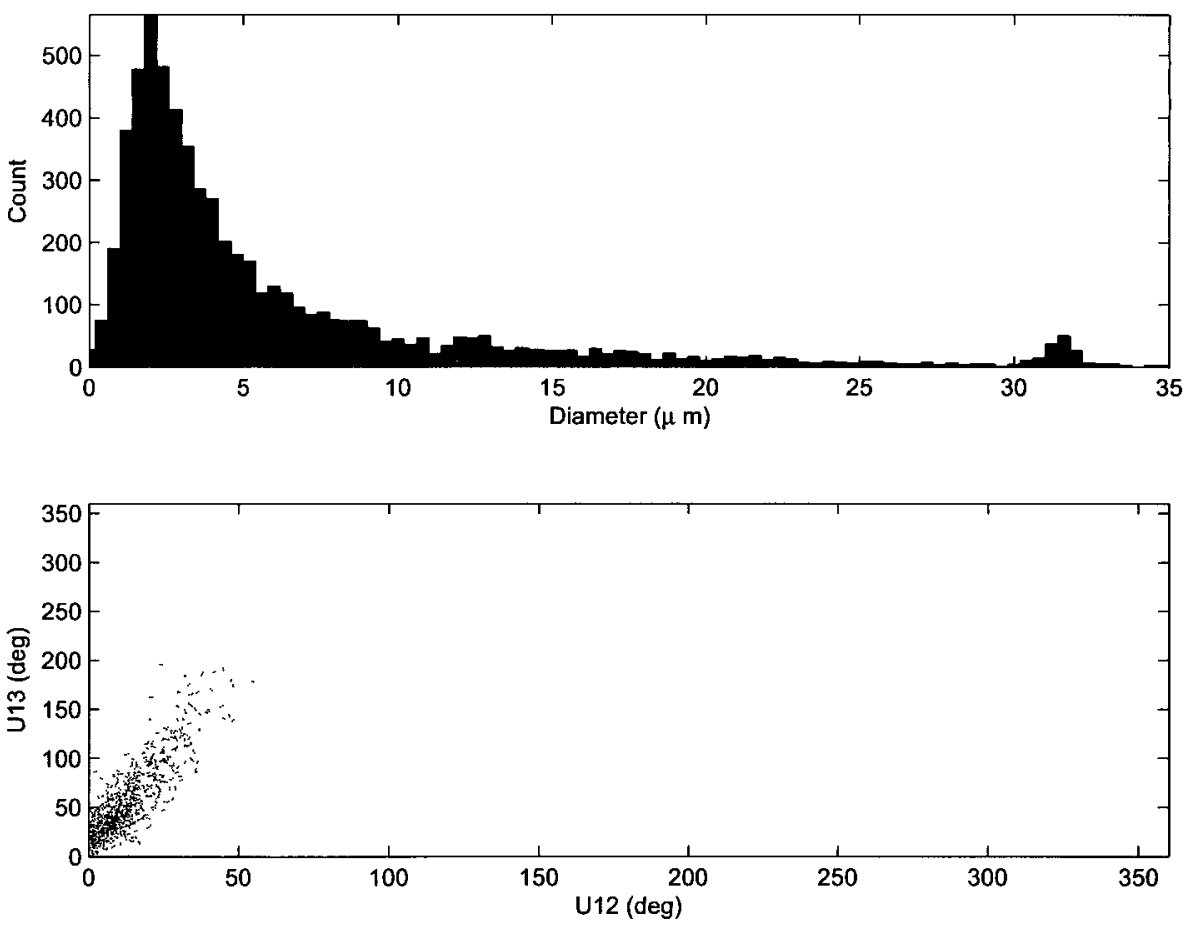

(b) Diameter histogram and PDA detector phase validation

Figure B.7: $\mathrm{x}=35 \mathrm{~mm}, \mathrm{r}=5 \mathrm{~mm}$ 

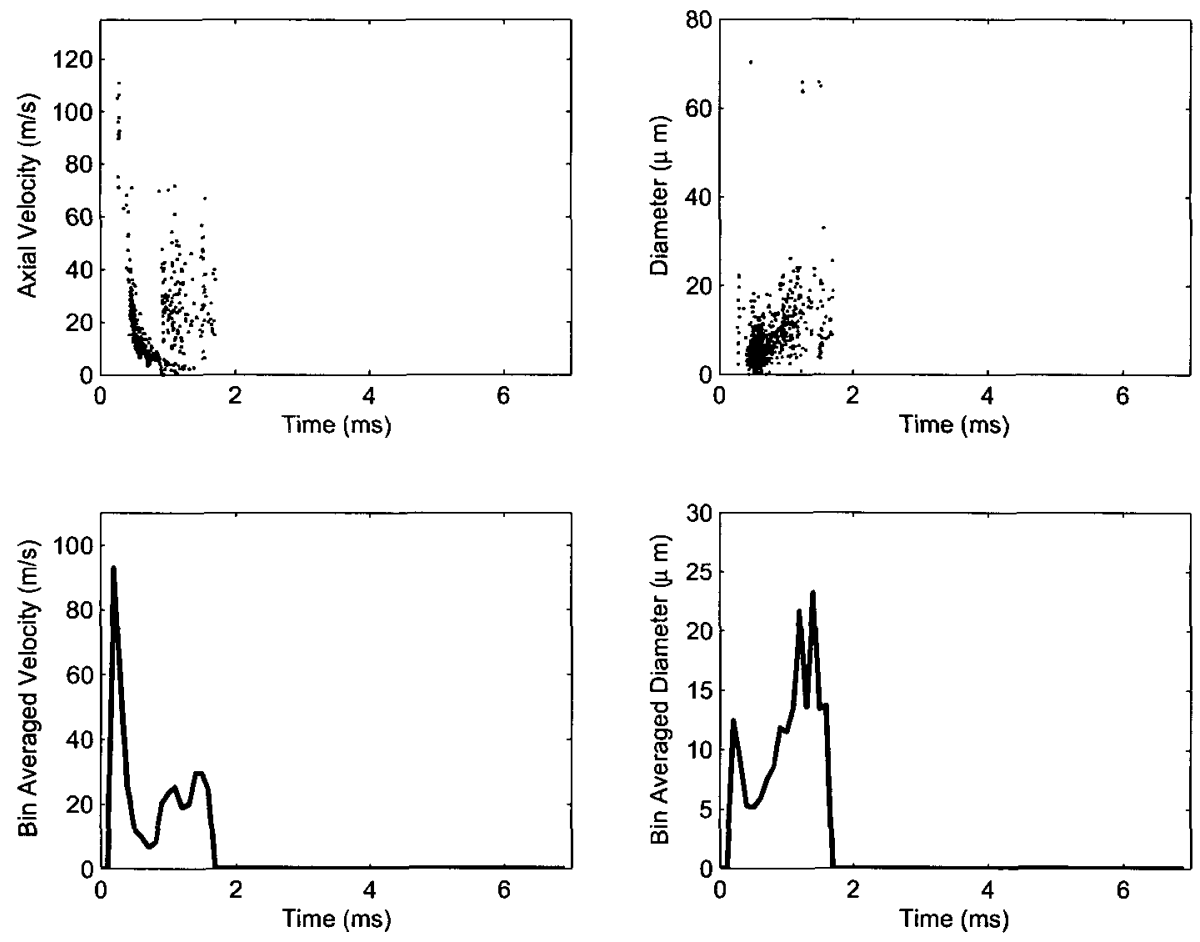

(a) Axial velocity and diameter vs time
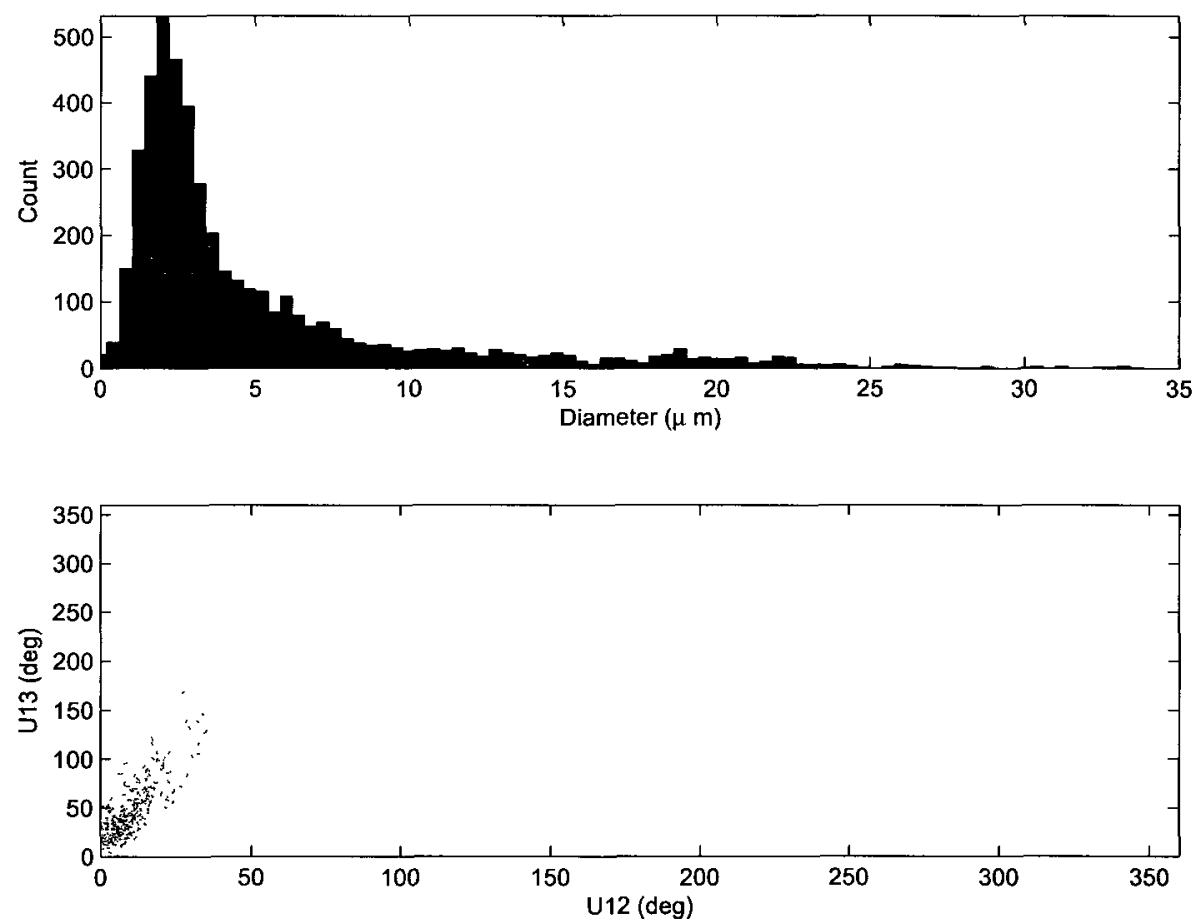

(b) Diameter histogram and PDA detector phase validation

Figure B.8: $\mathrm{x}=35 \mathrm{~mm}, \mathrm{r}=6 \mathrm{~mm}$ 


\section{B.1.2 $x=55 \mathrm{~mm}$}

The following pages illustrates results $55 \mathrm{~mm}$ away from the nozzle tip: 

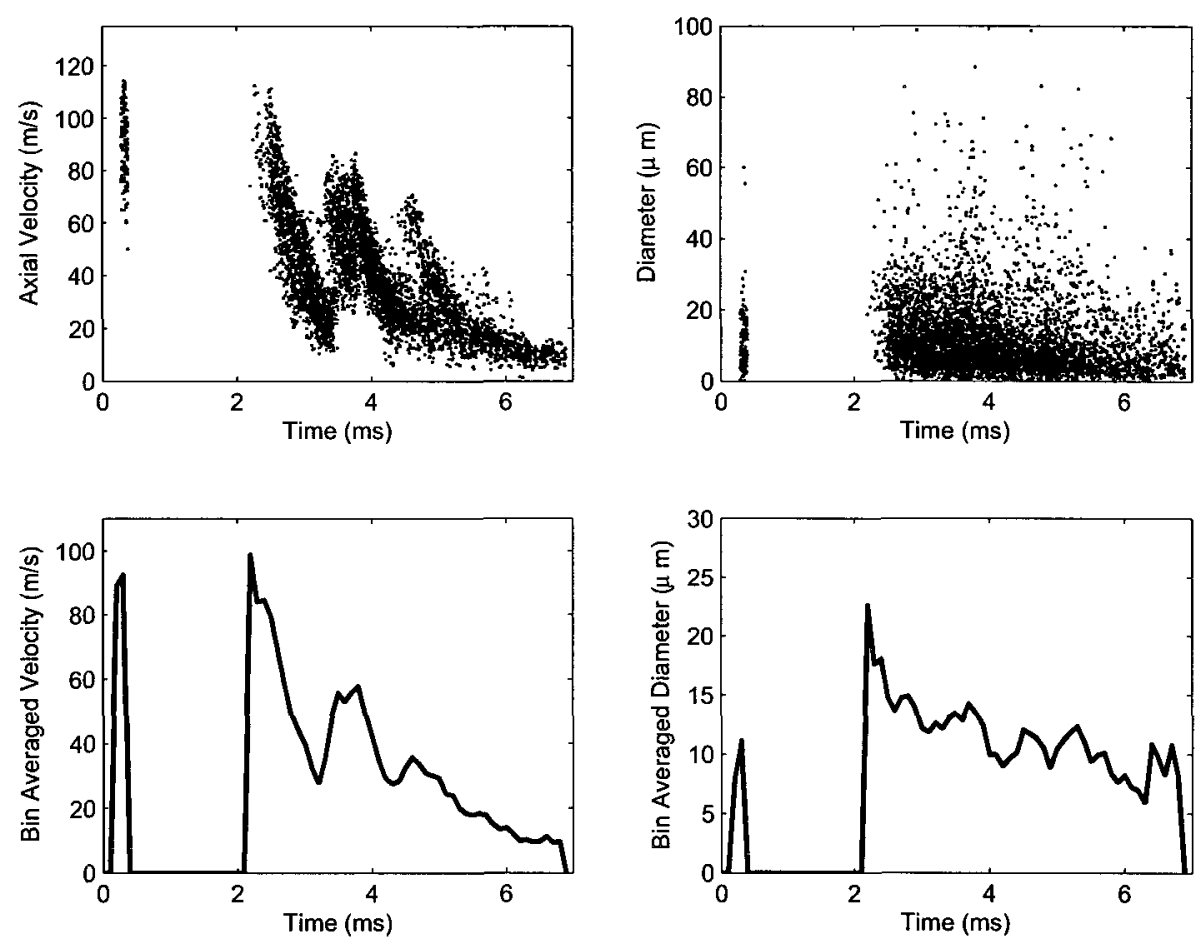

(a) Axial velocity and diameter vs time
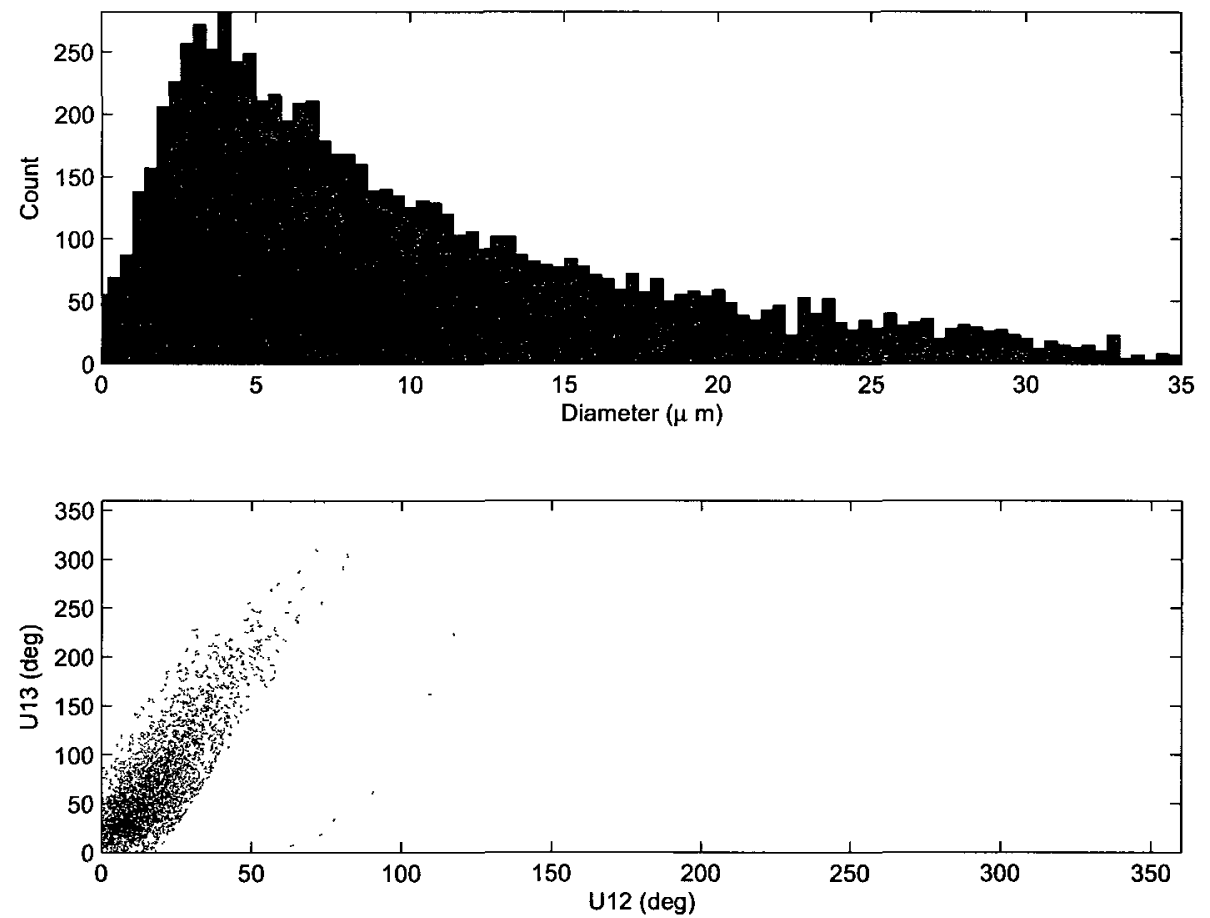

(b) Diameter histogram and PDA detector phase validation

Figure B.9: $\mathrm{x}=55 \mathrm{~mm}, \mathrm{r}=0 \mathrm{~mm}$ 

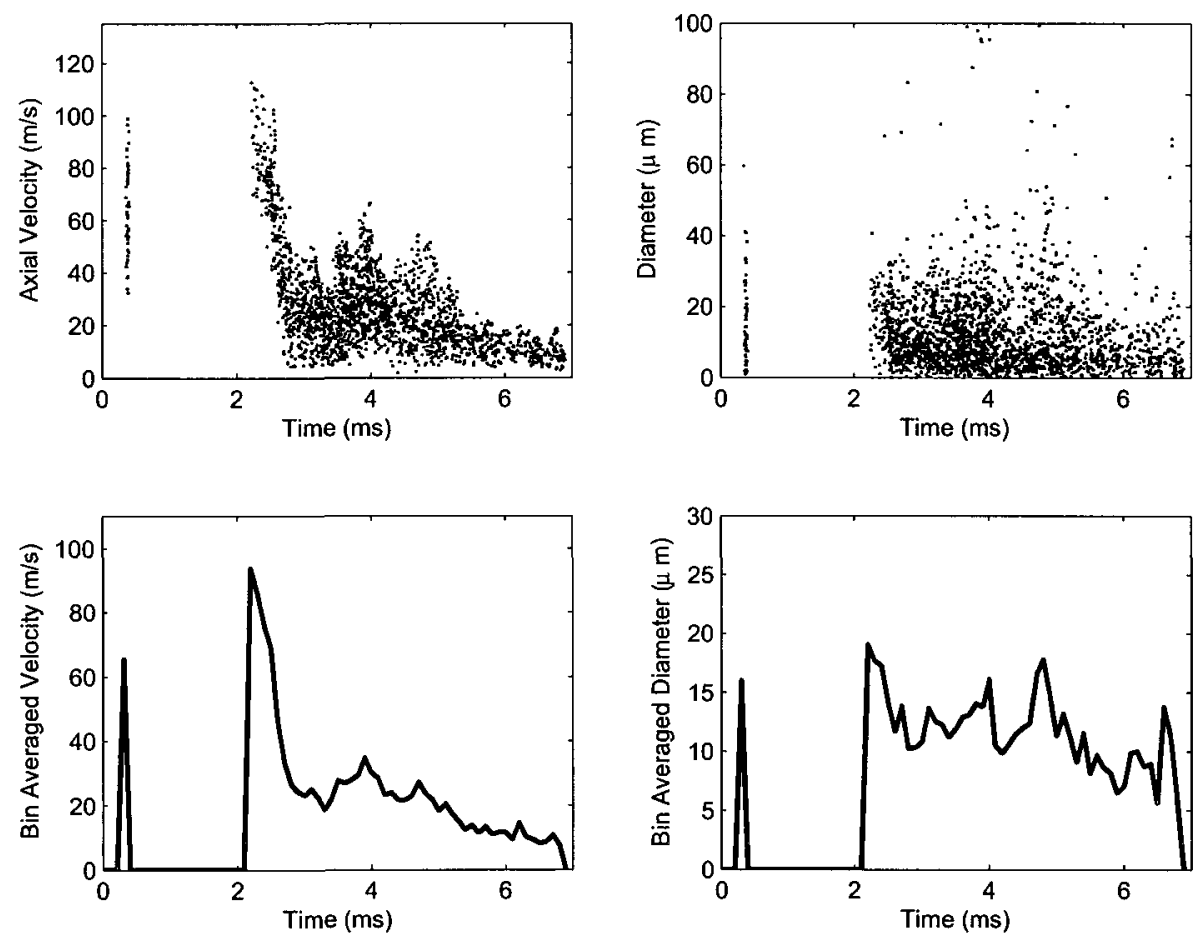

(a) Axial velocity and diameter vs time
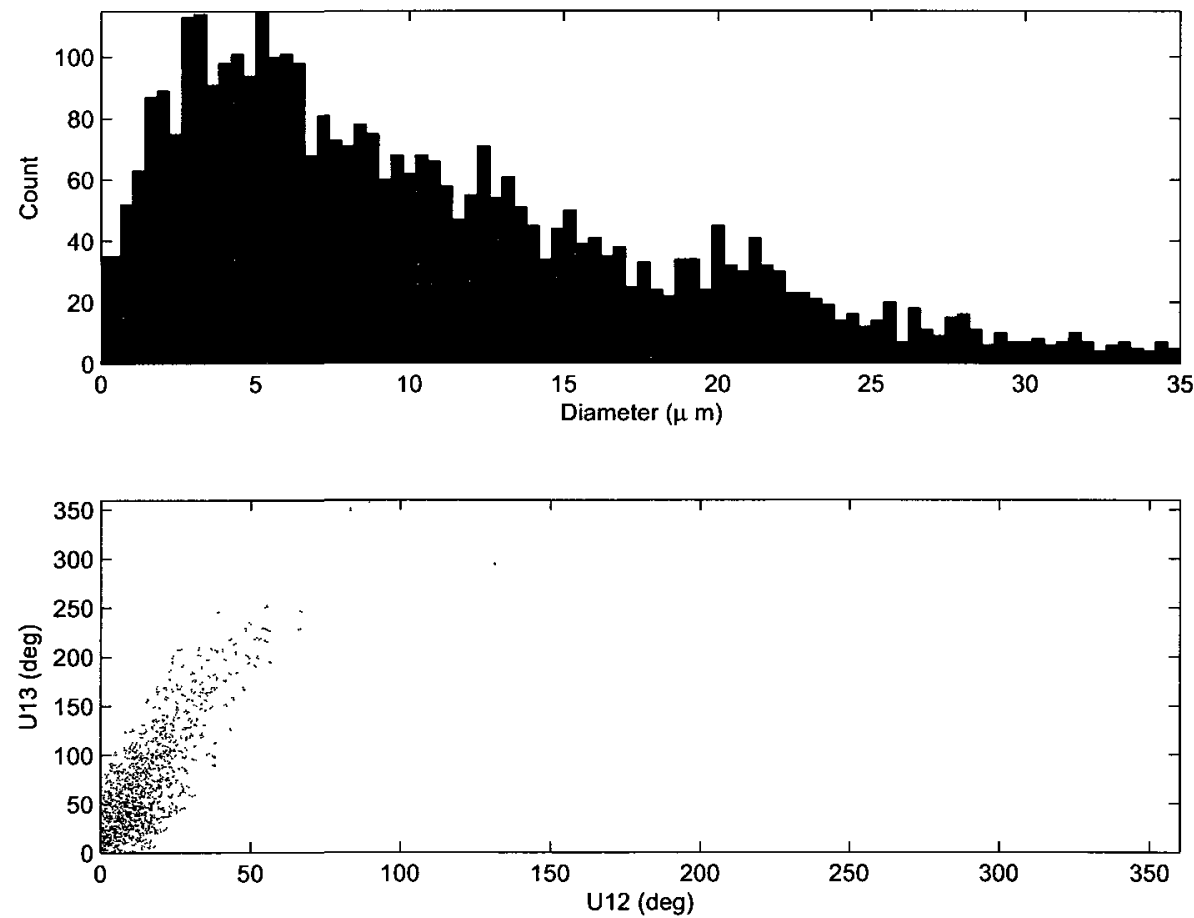

(b) Diameter histogram and PDA detector phase validation

Figure B.10: $\mathrm{x}=55 \mathrm{~mm}, \mathrm{r}=2.5 \mathrm{~mm}$ 

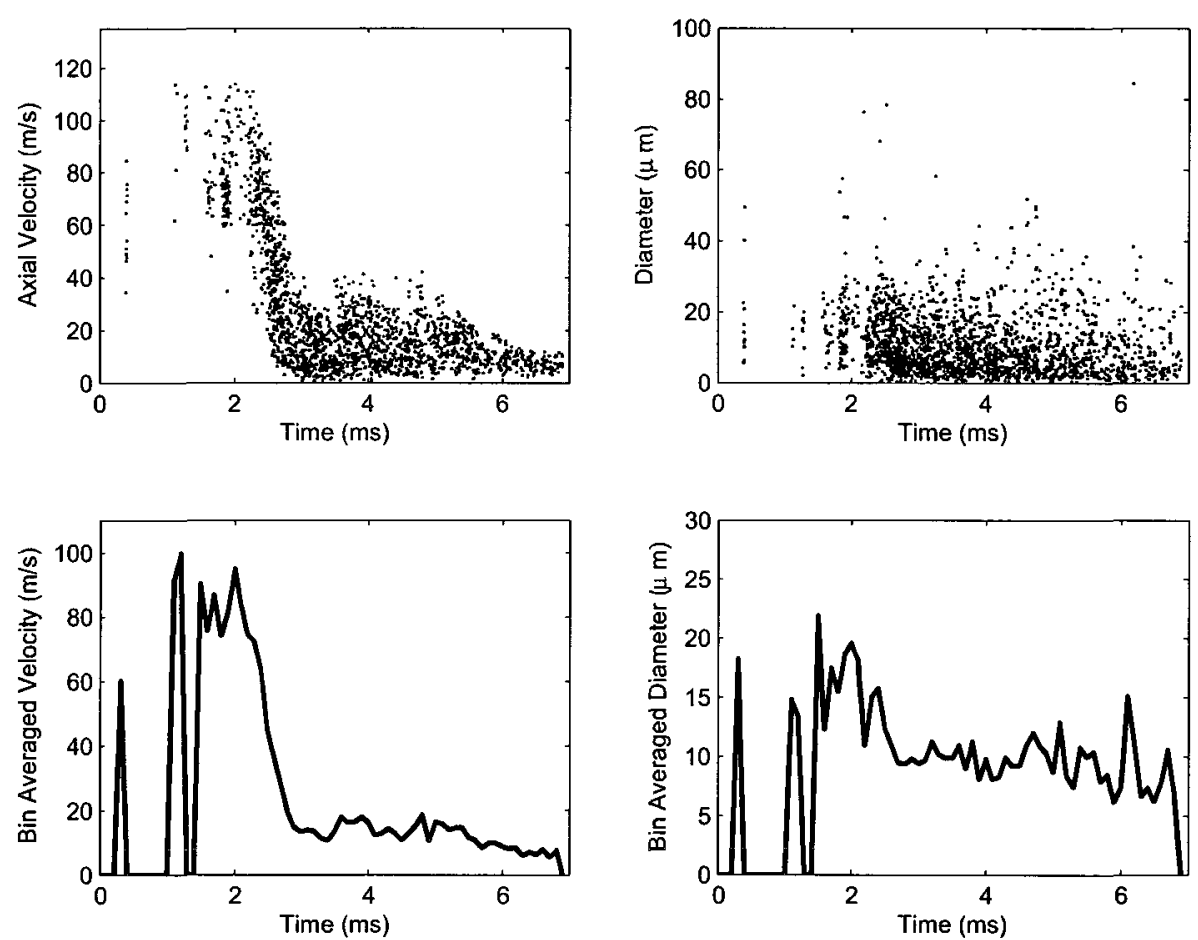

(a) Axial velocity and diameter vs time
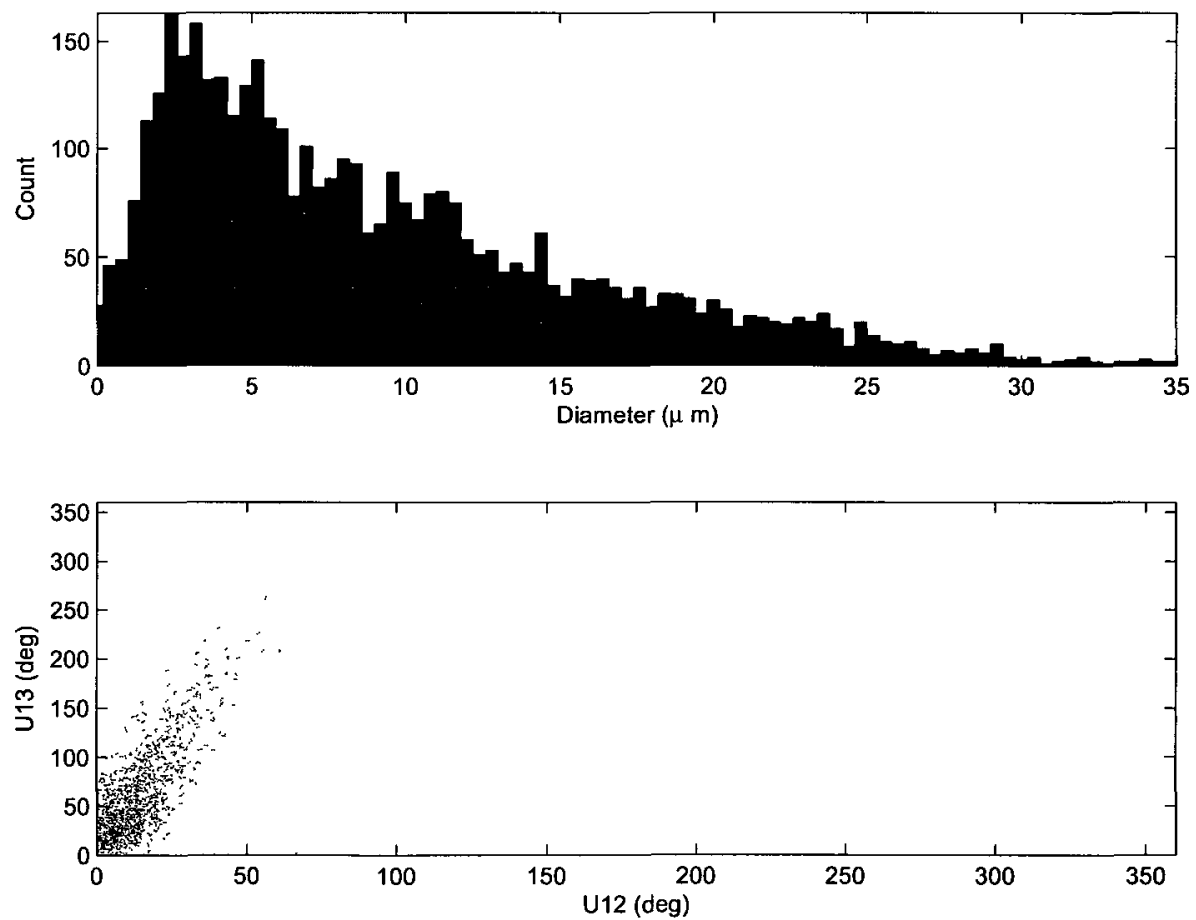

(b) Diameter histogram and PDA detector phase validation

Figure B.11: $\mathrm{x}=55 \mathrm{~mm}, \mathrm{r}=3.5 \mathrm{~mm}$ 

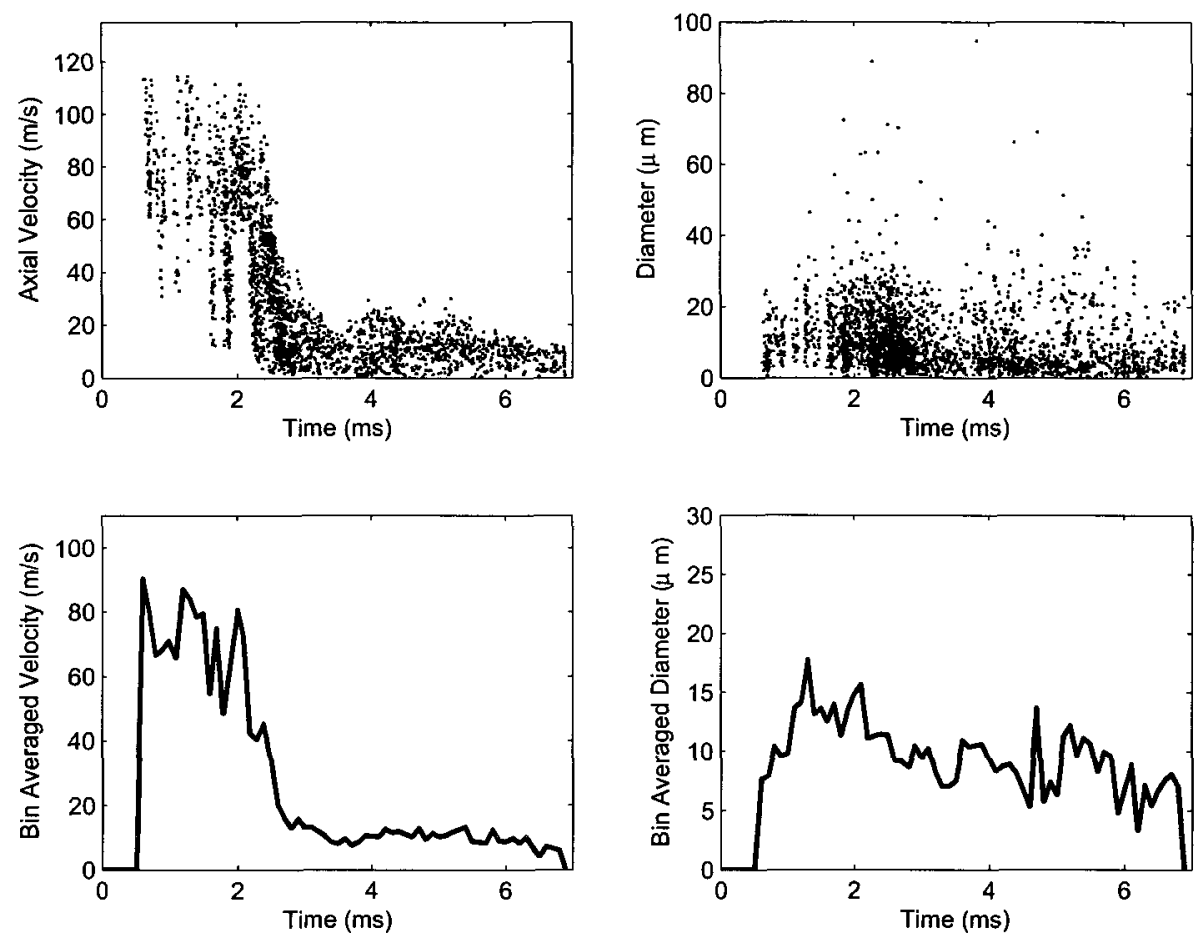

(a) Axial velocity and diameter vs time
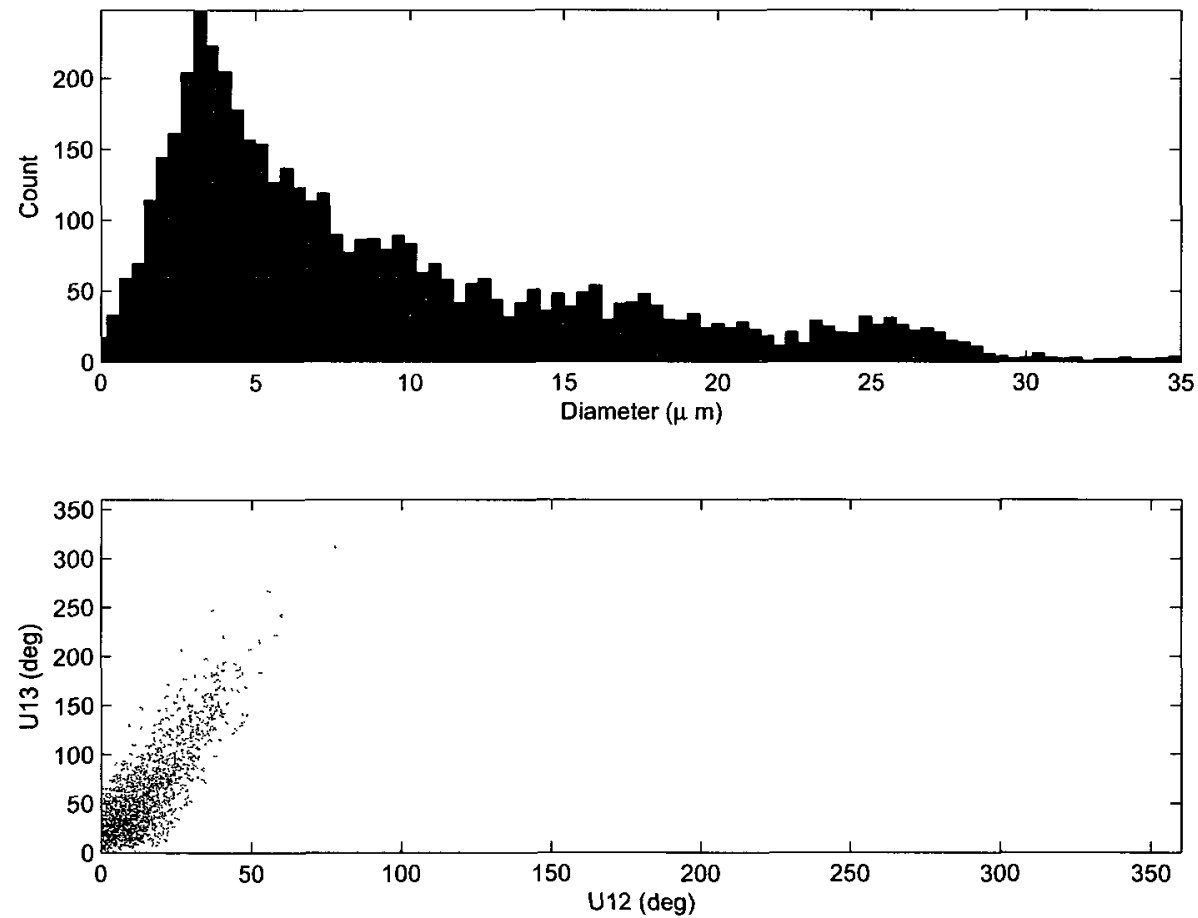

(b) Diameter histogram and PDA detector phase validation

Figure B.12: $\mathrm{x}=55 \mathrm{~mm}, \mathrm{r}=4.5 \mathrm{~mm}$ 

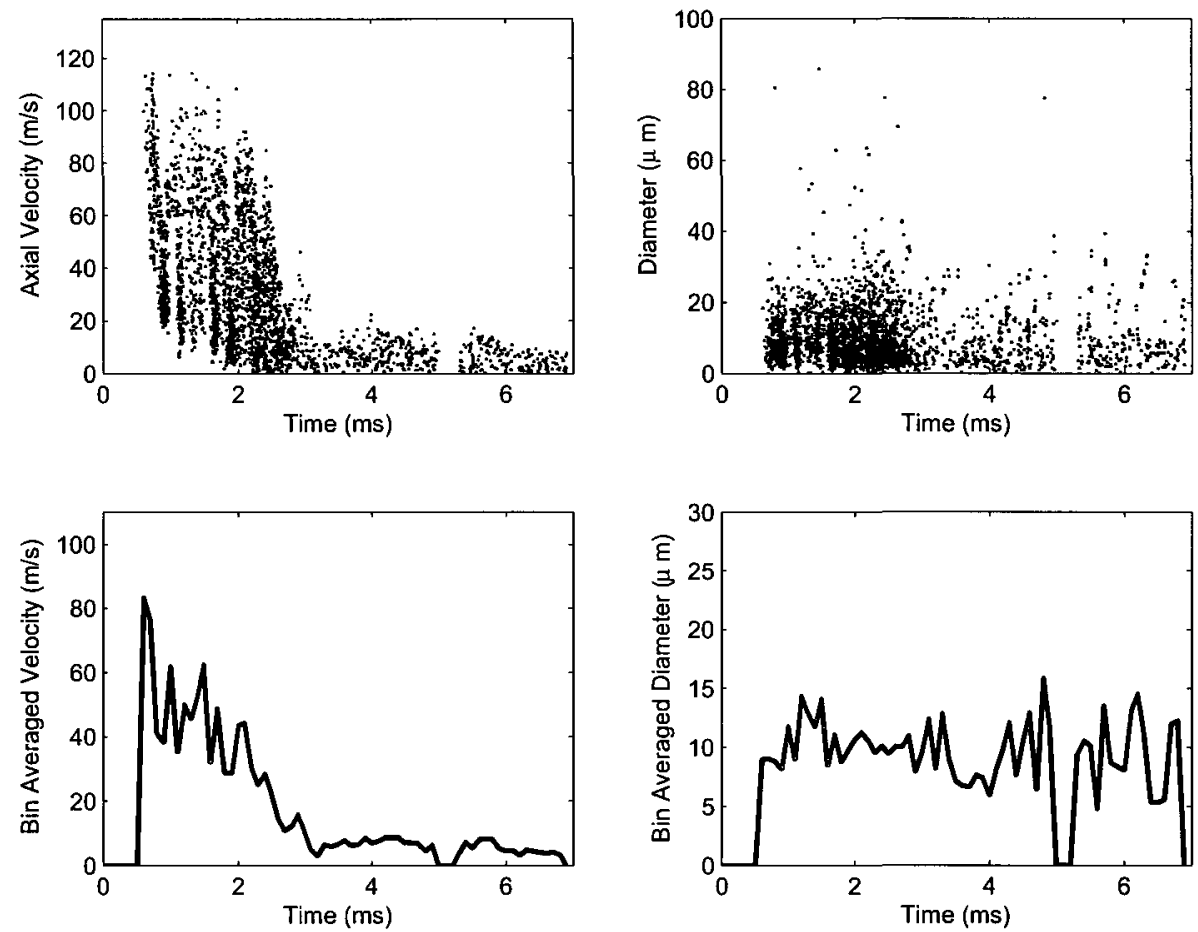

(a) Axial velocity and diameter vs time
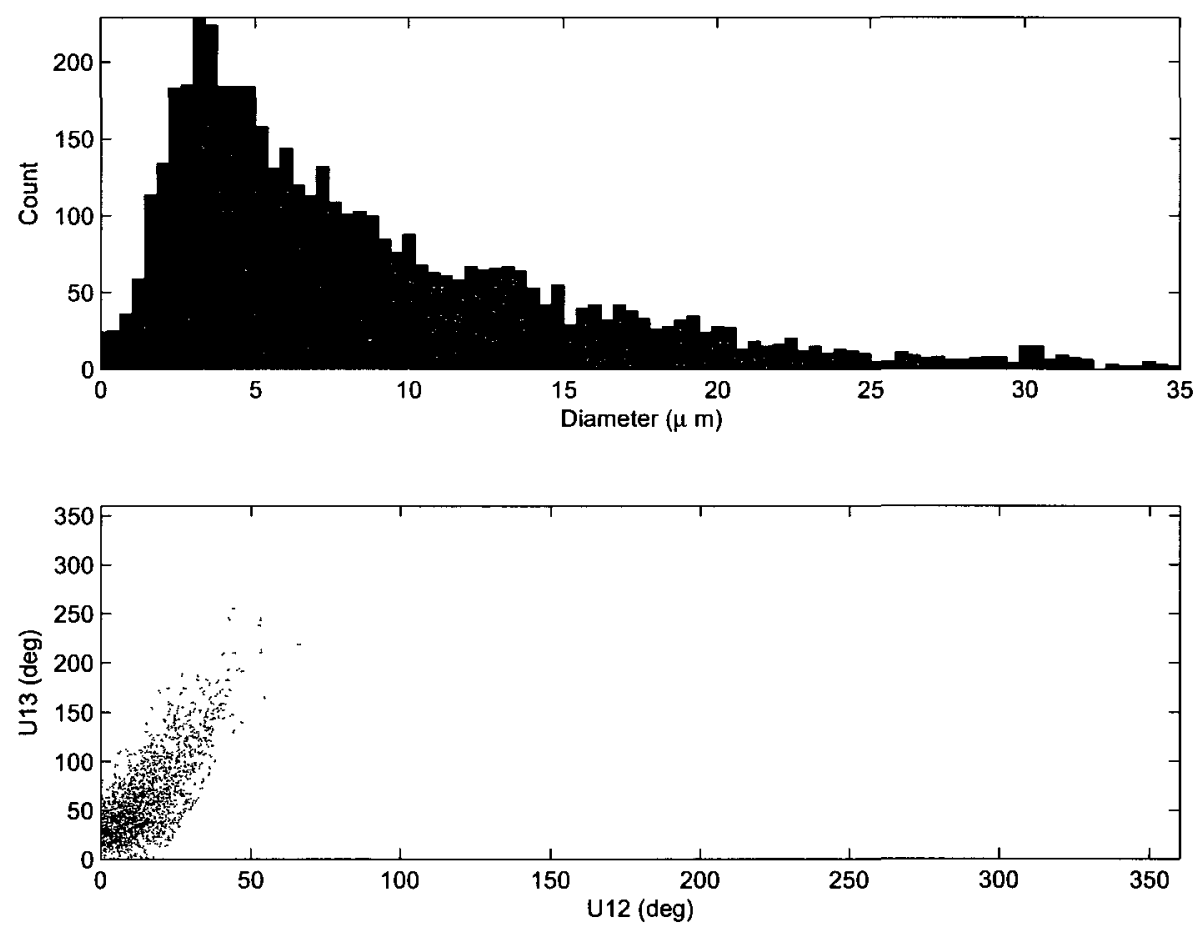

(b) Diameter histogram and PDA detector phase validation

Figure B.13: $\mathrm{x}=55 \mathrm{~mm}, \mathrm{r}=5.5 \mathrm{~mm}$ 

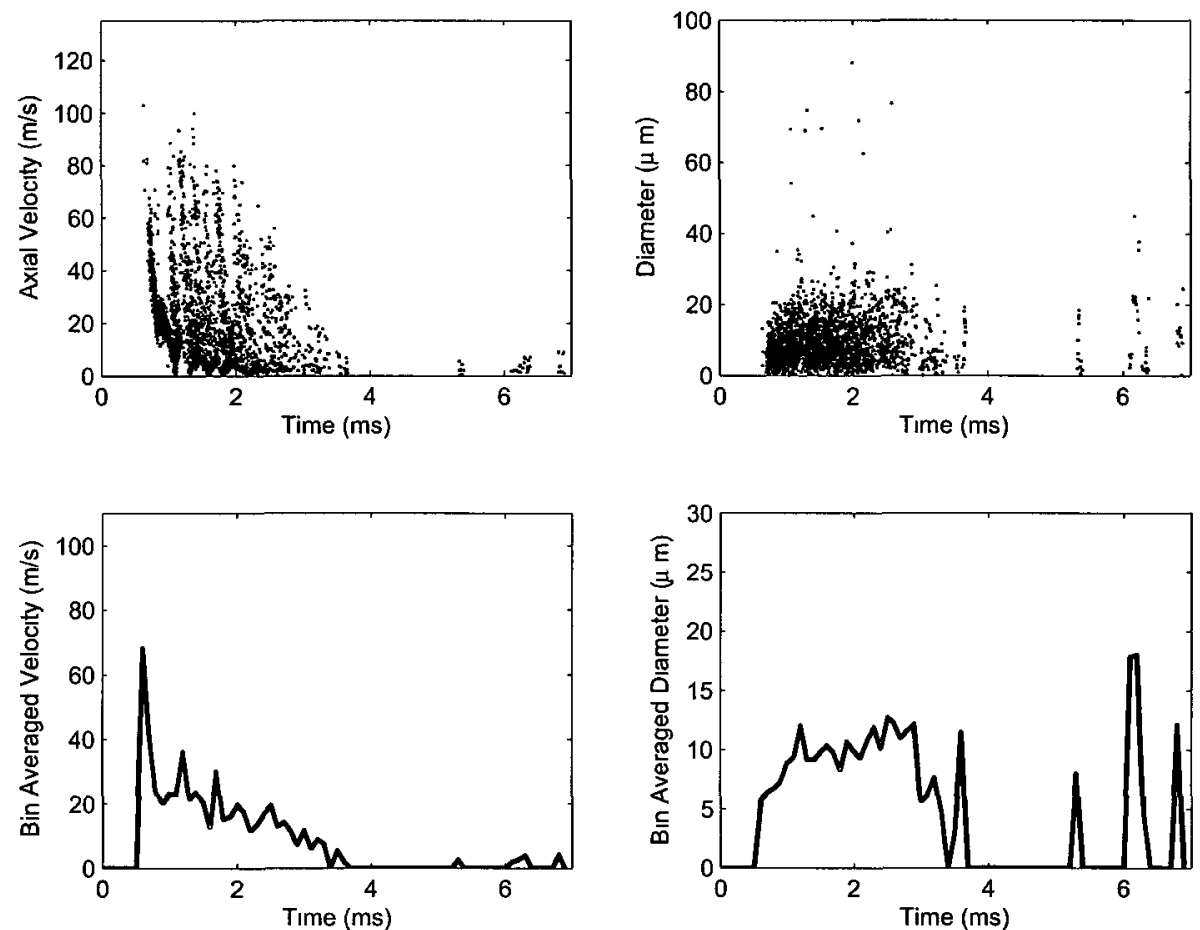

(a) Axial velocity and diameter vs time
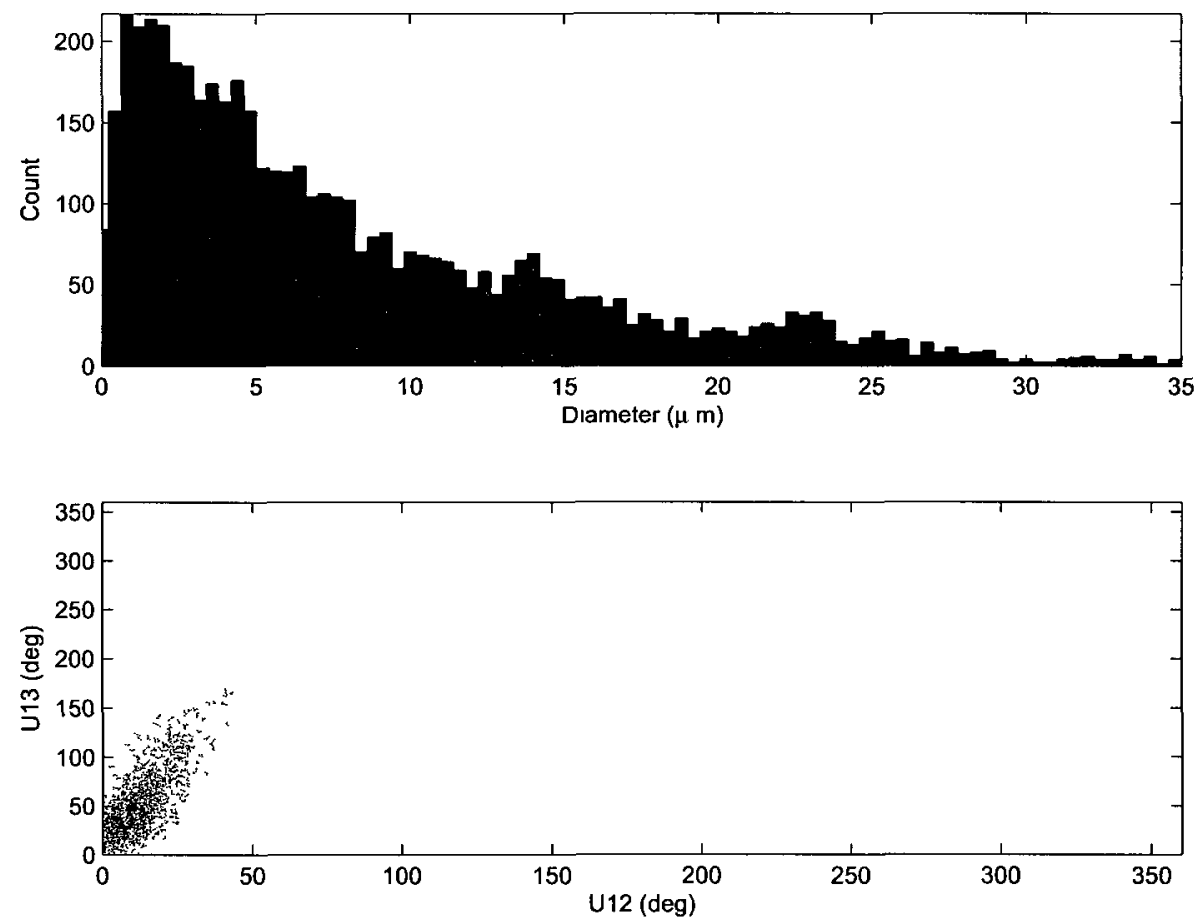

(b) Diameter histogram and PDA detector phase validation

Figure B.14: $\mathrm{x}=55 \mathrm{~mm}, \mathrm{r}=6.5 \mathrm{~mm}$ 

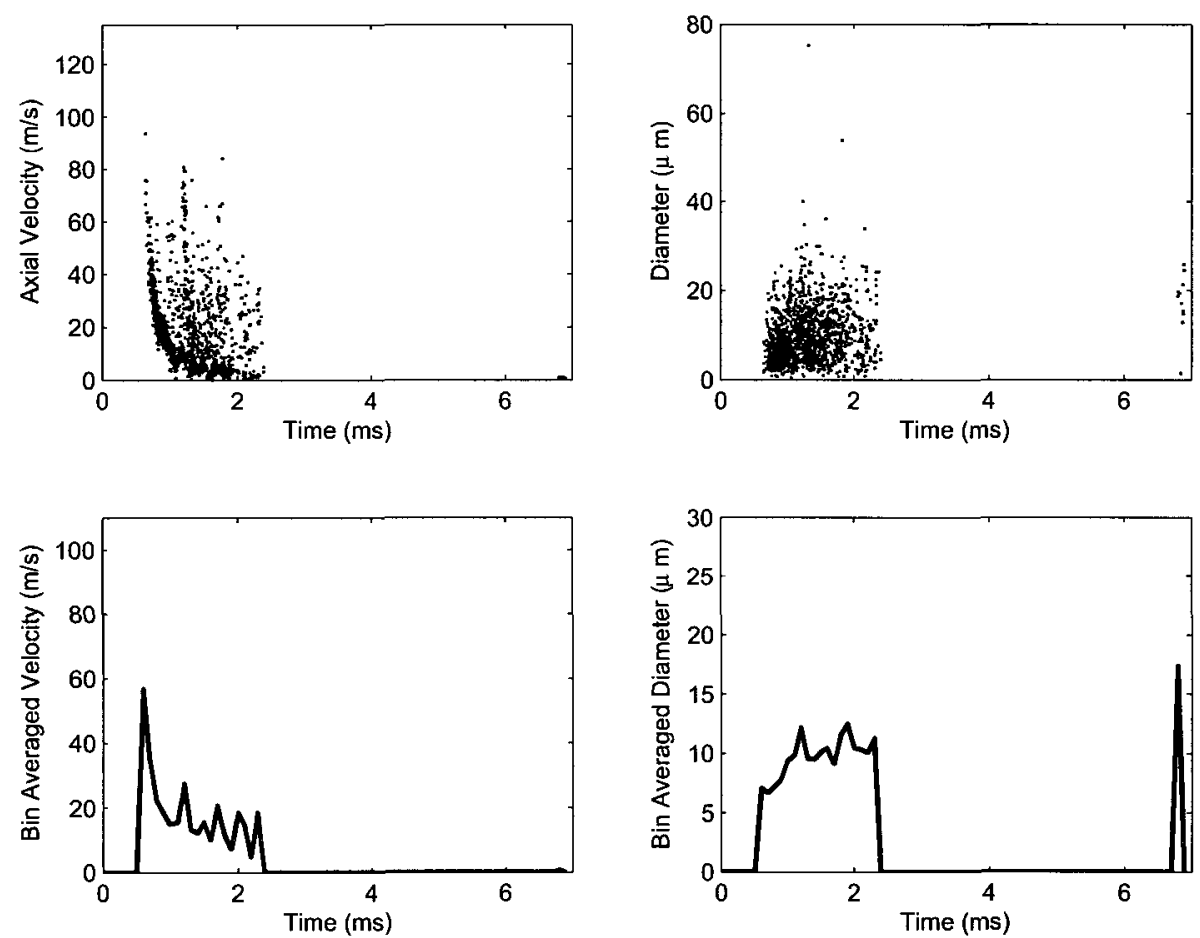

(a) Axial velocity and diameter vs time
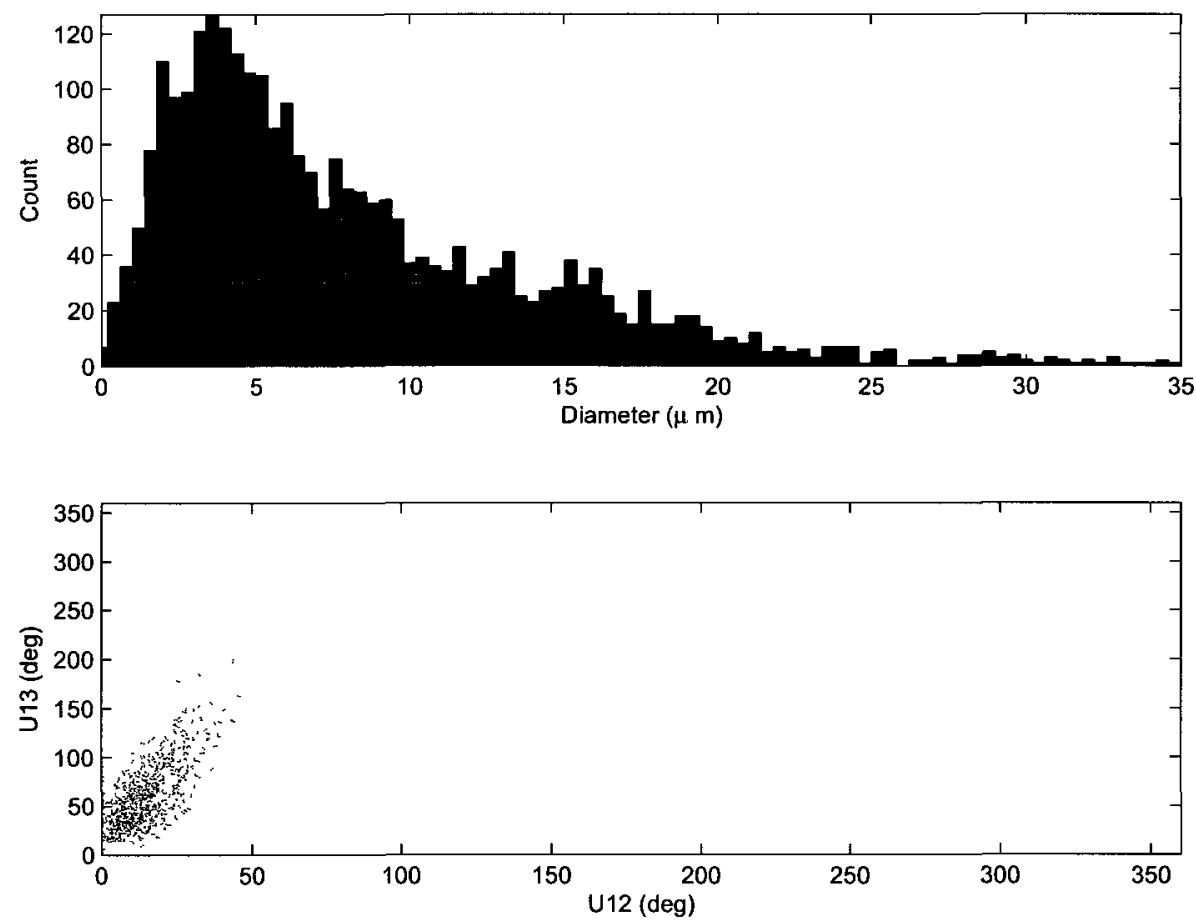

(b) Diameter histogram and PDA detector phase validation

Figure B.15: $x=55 \mathrm{~mm}, \mathrm{r}=7.5 \mathrm{~mm}$ 

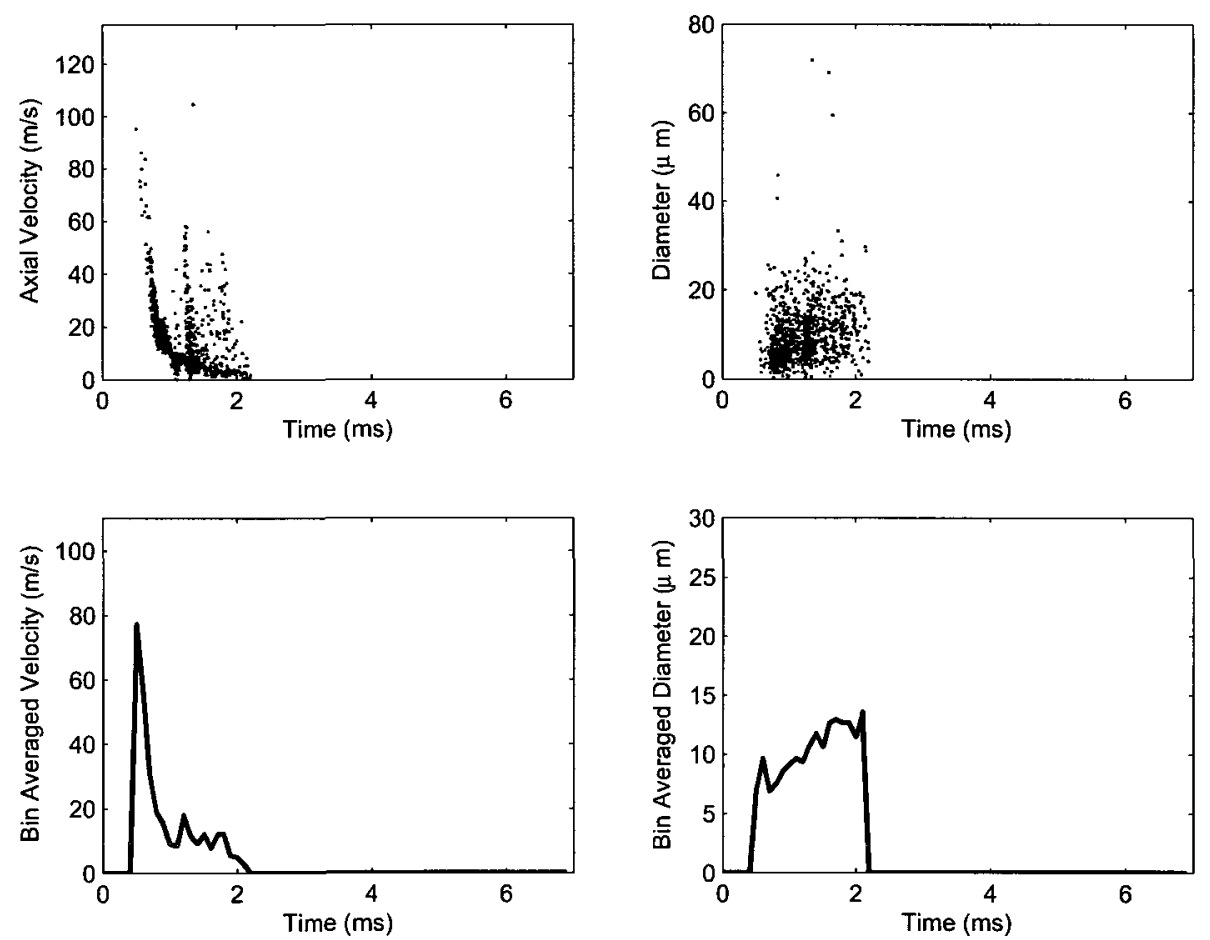

(a) Axial velocity and diameter vs time
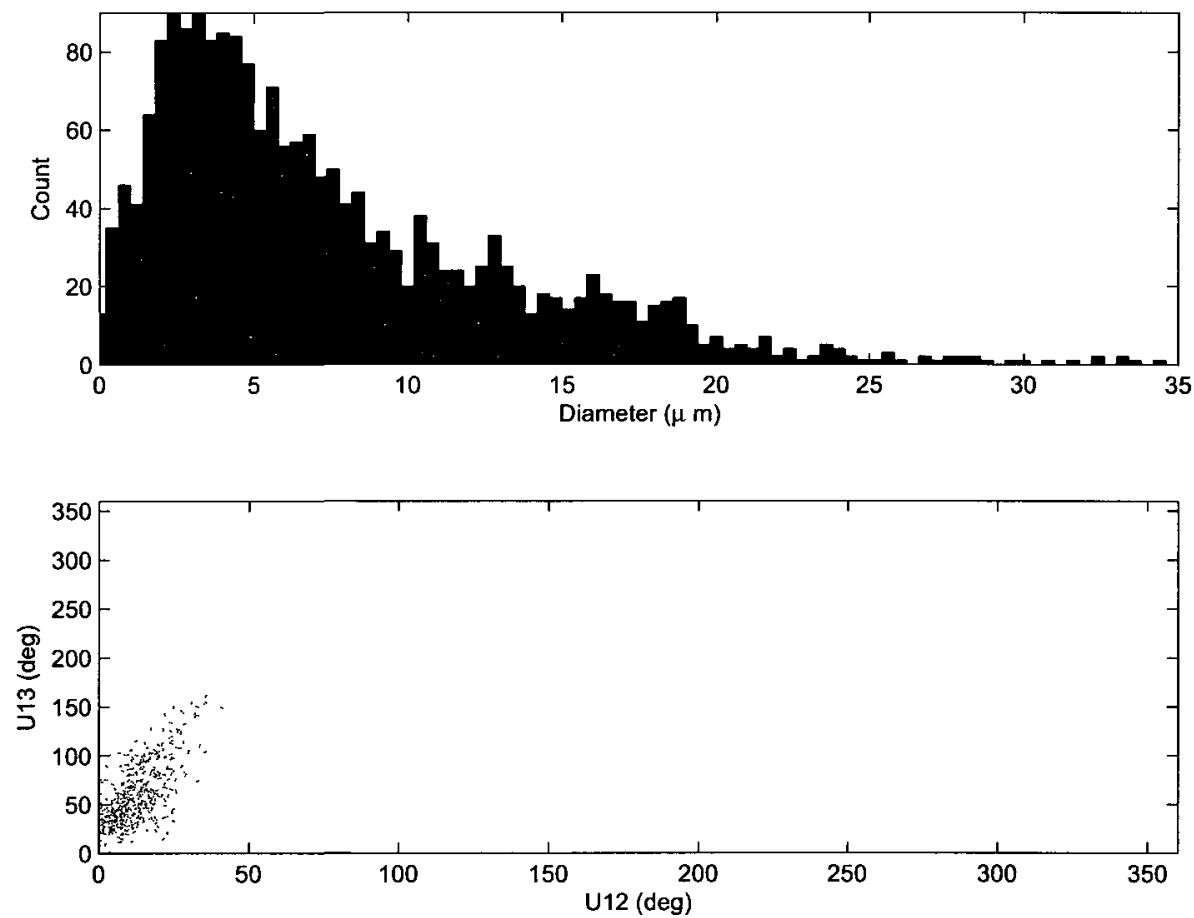

(b) Diameter histogram and PDA detector phase validation

Figure B.16: $\mathrm{x}=55 \mathrm{~mm}, \mathrm{r}=8.5 \mathrm{~mm}$ 


\section{B.1.3 $x=70 \mathrm{~mm}$}

The following pages illustrates results $70 \mathrm{~mm}$ away from the nozzle tip: 

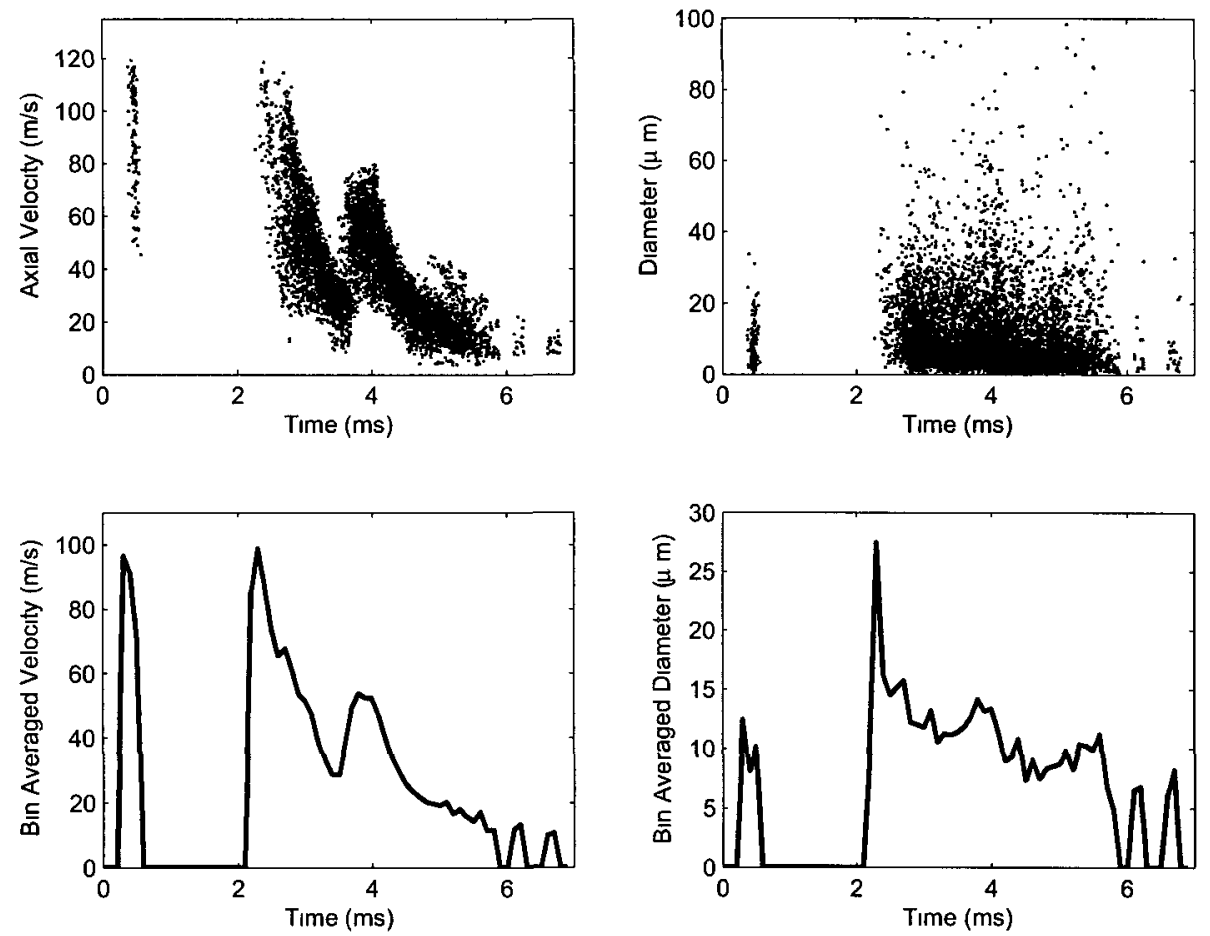

(a) Axial velocity and diameter vs time
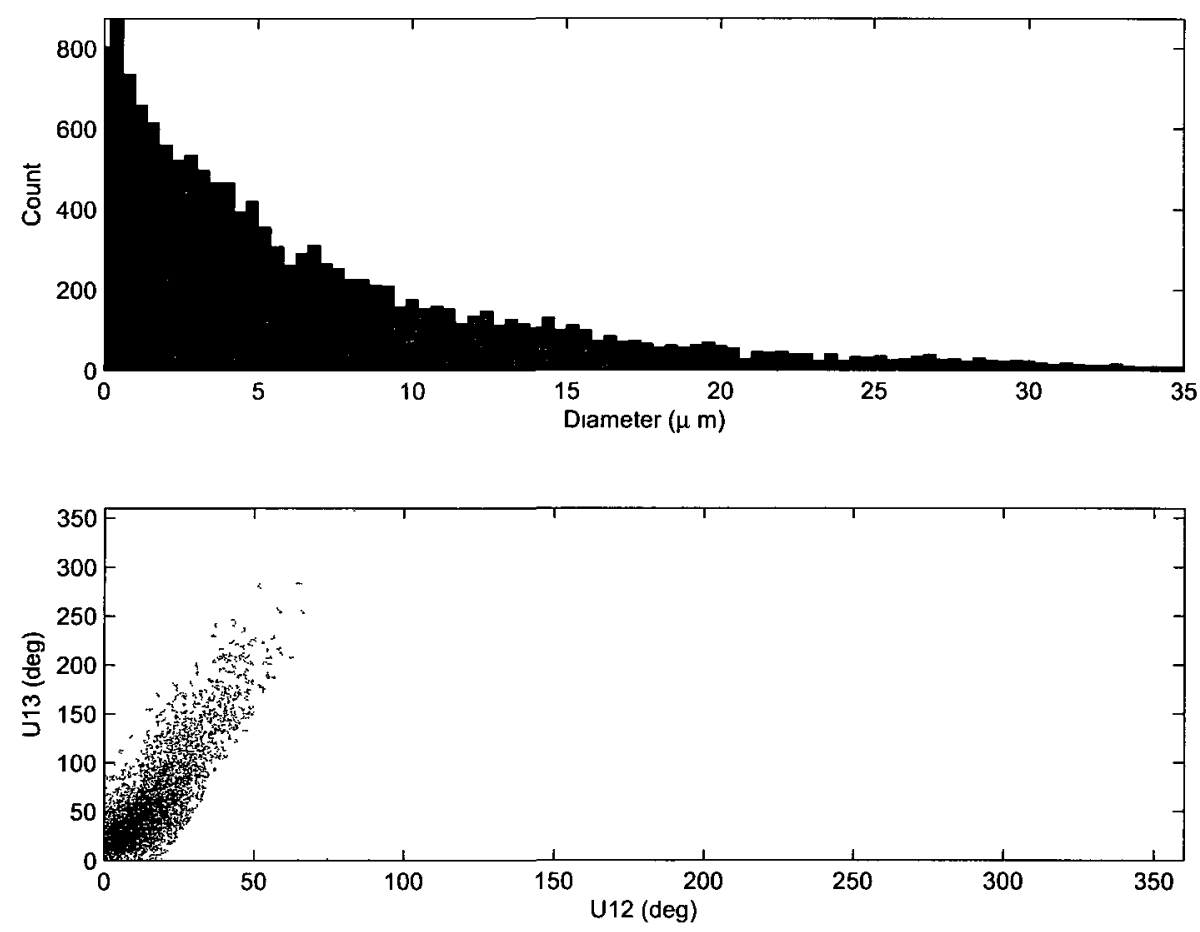

(b) Diameter histogram and PDA detector phase validation

Figure B.17: $\mathrm{x}=70 \mathrm{~mm}, \mathrm{r}=0 \mathrm{~mm}$ 

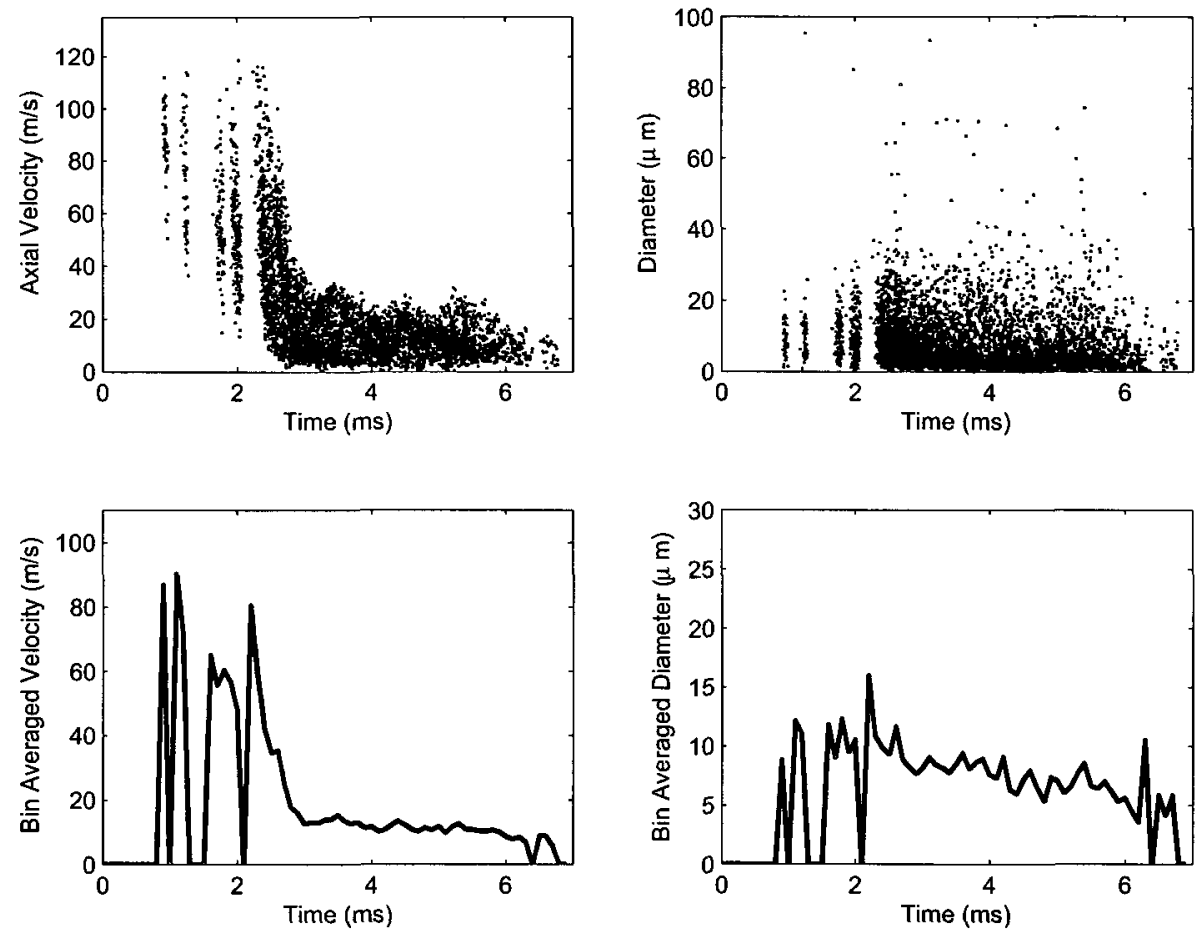

(a) Axial velocity and diameter vs time
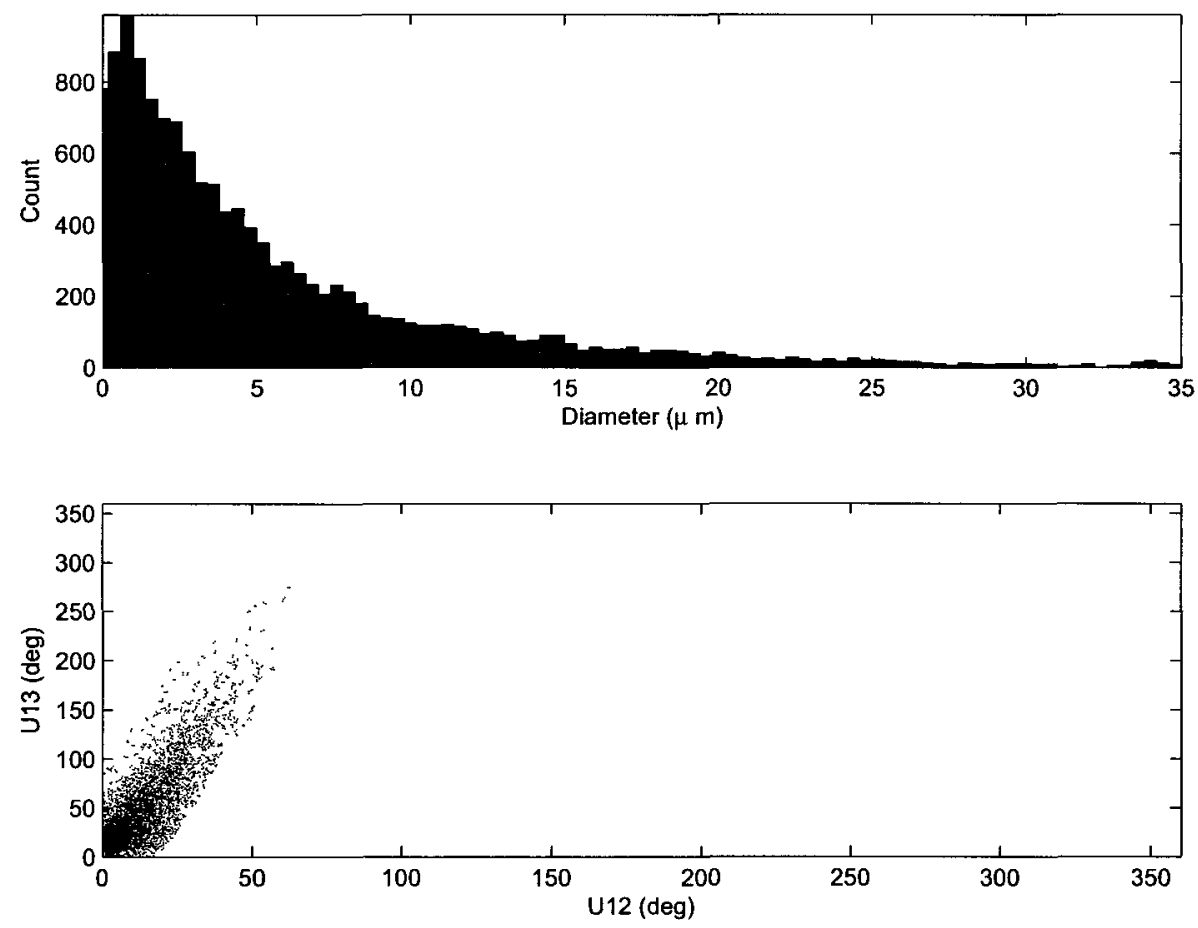

(b) Diameter histogram and PDA detector phase validation

Figure B.18: $\mathrm{x}=70 \mathrm{~mm}, \mathrm{r}=4.5 \mathrm{~mm}$ 

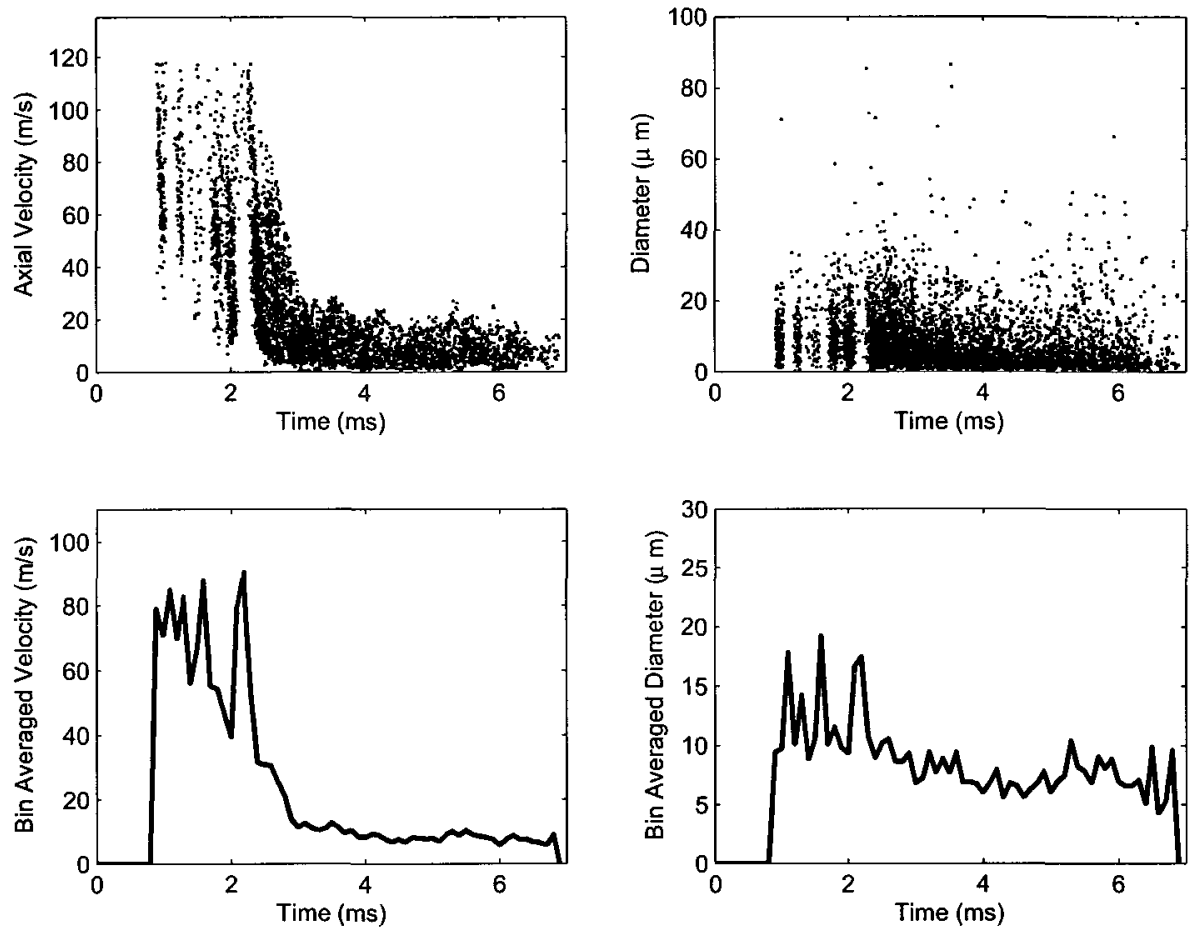

(a) Axial velocity and diameter vs time
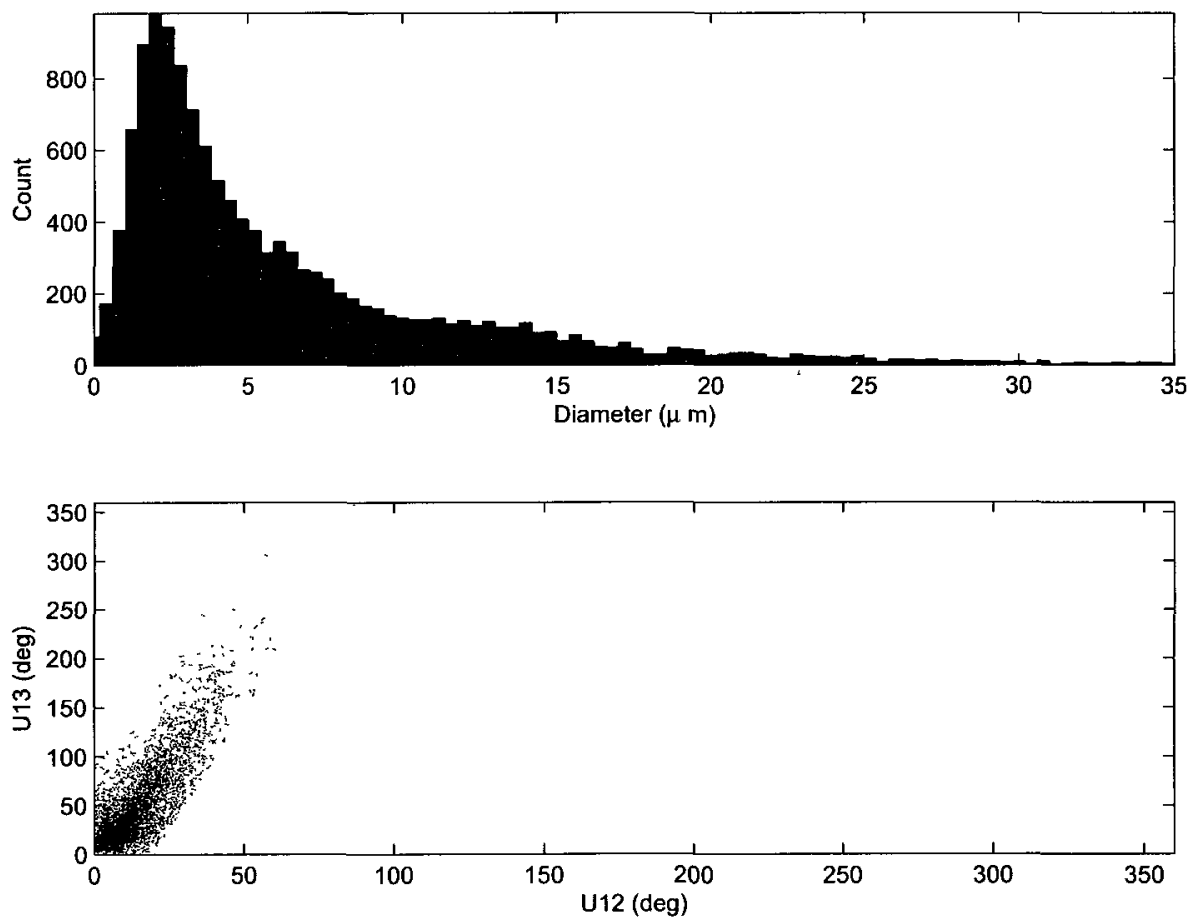

(b) Diameter histogram and PDA detector phase validation

Figure B.19: $x=70 \mathrm{~mm}, \mathrm{r}=5.5 \mathrm{~mm}$ 

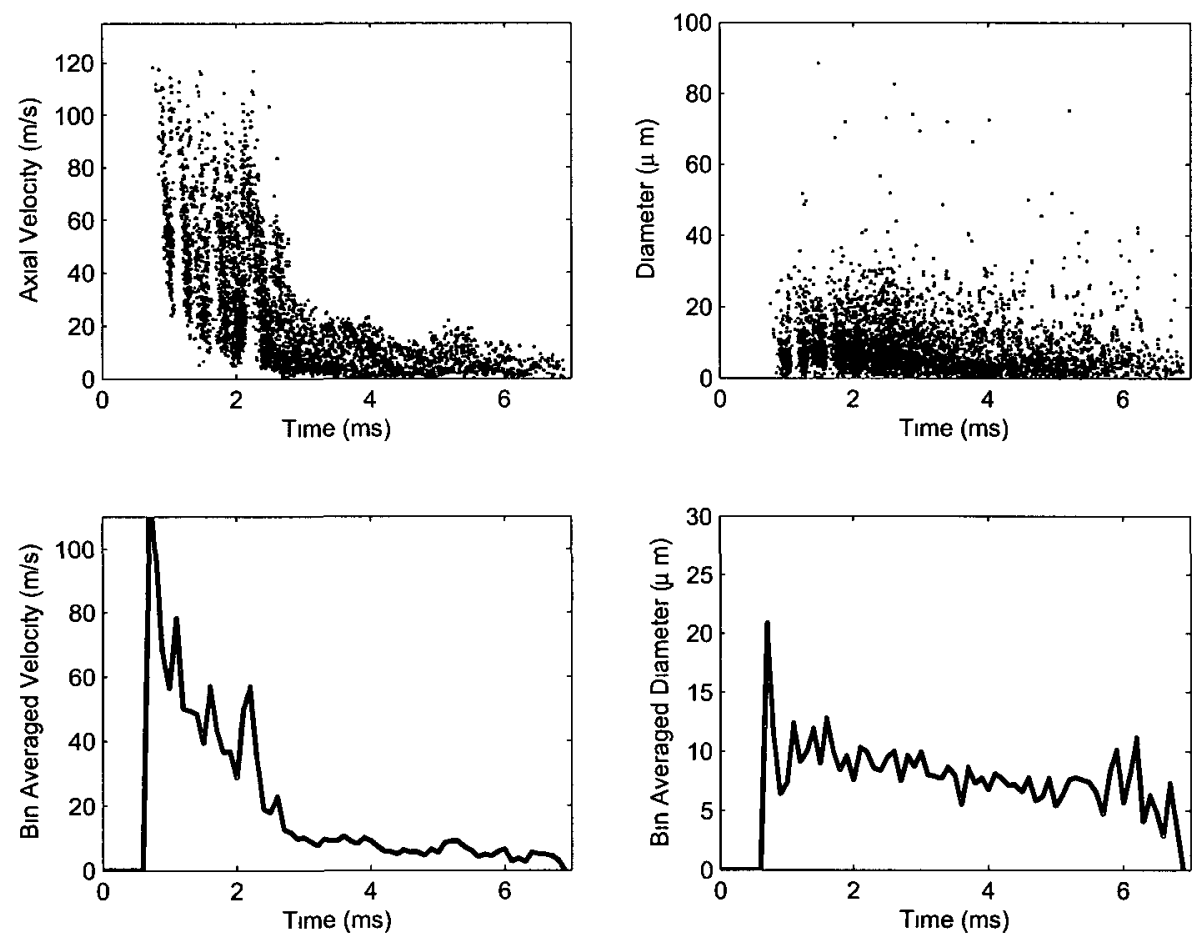

(a) Axial velocity and diameter vs time
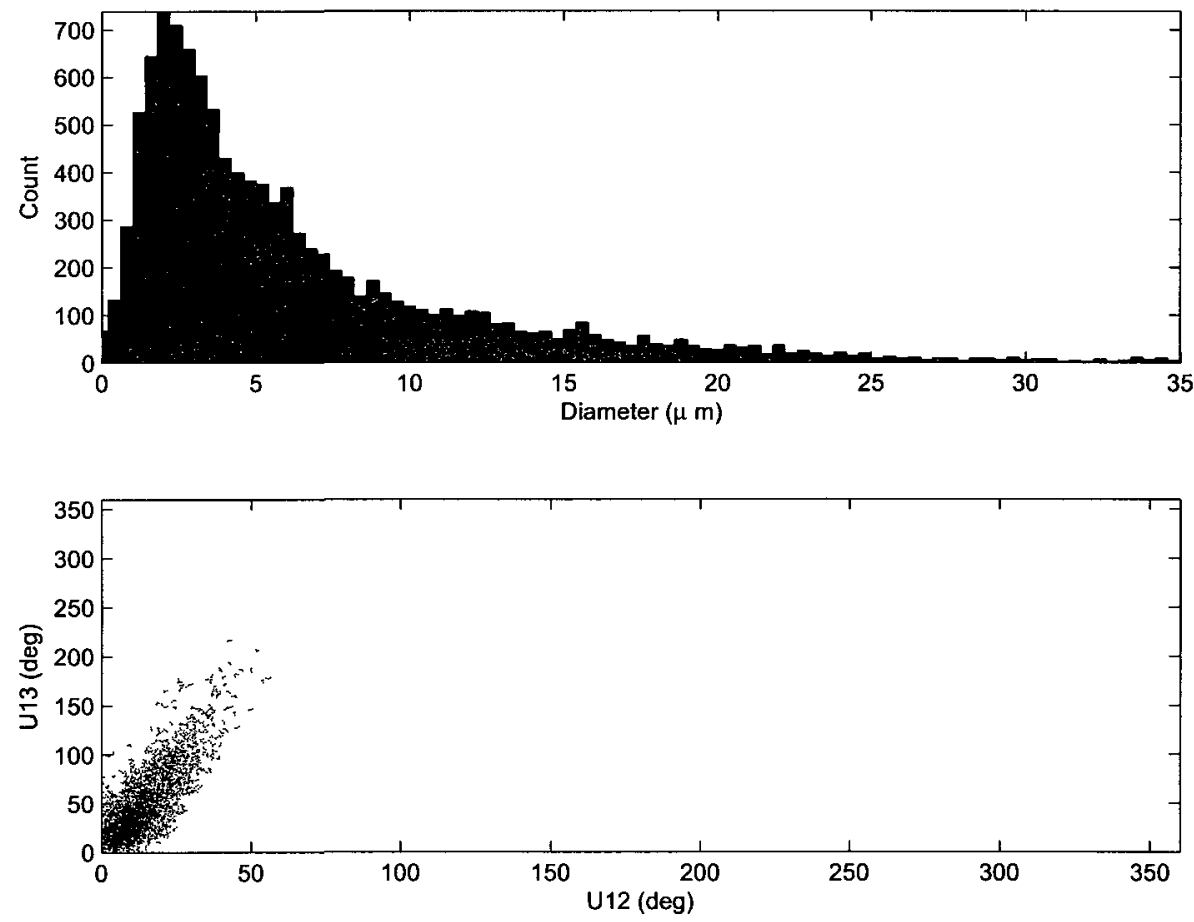

(b) Diameter histogram and PDA detector phase validation

Figure B.20: $\mathrm{x}=70 \mathrm{~mm}, \mathrm{r}=6.5 \mathrm{~mm}$ 

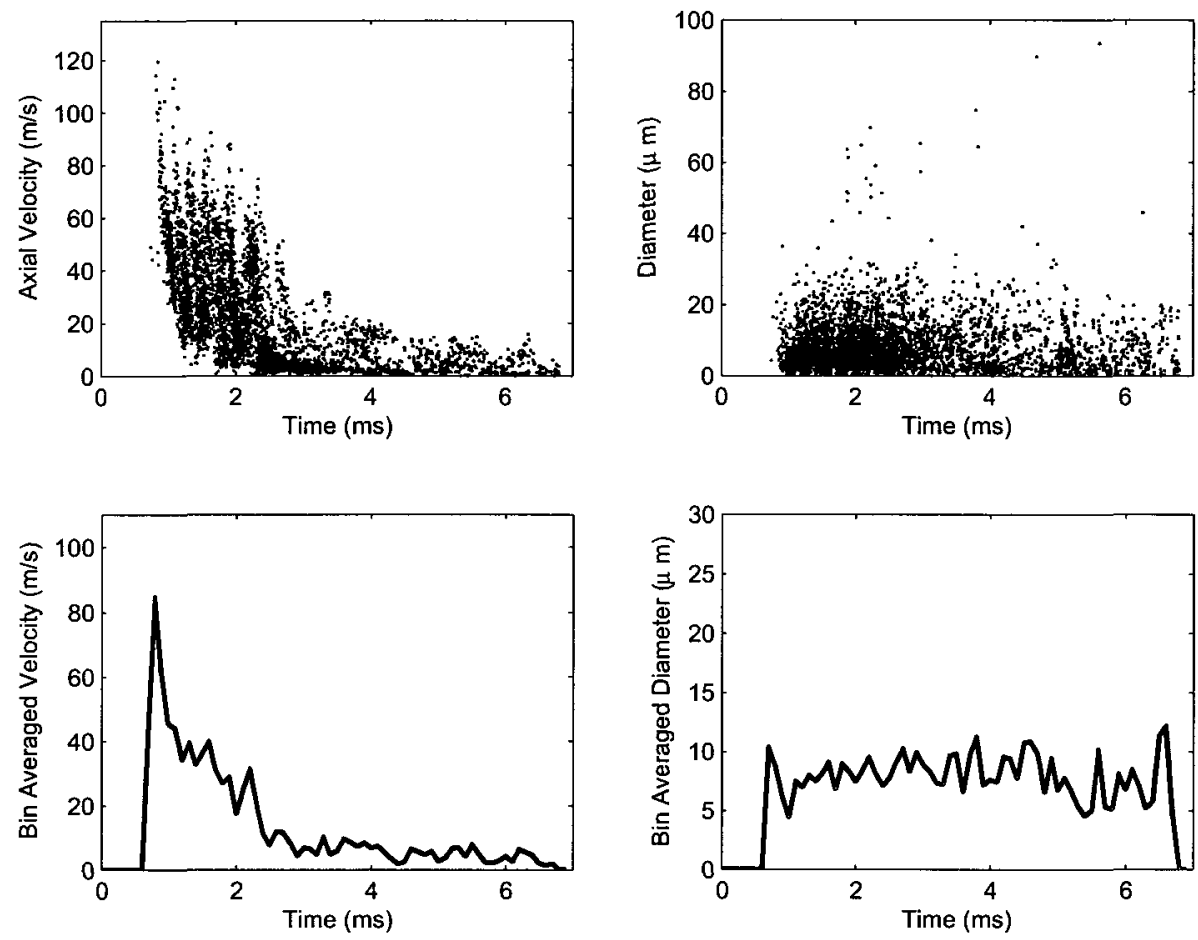

(a) Axial velocity and diameter vs time
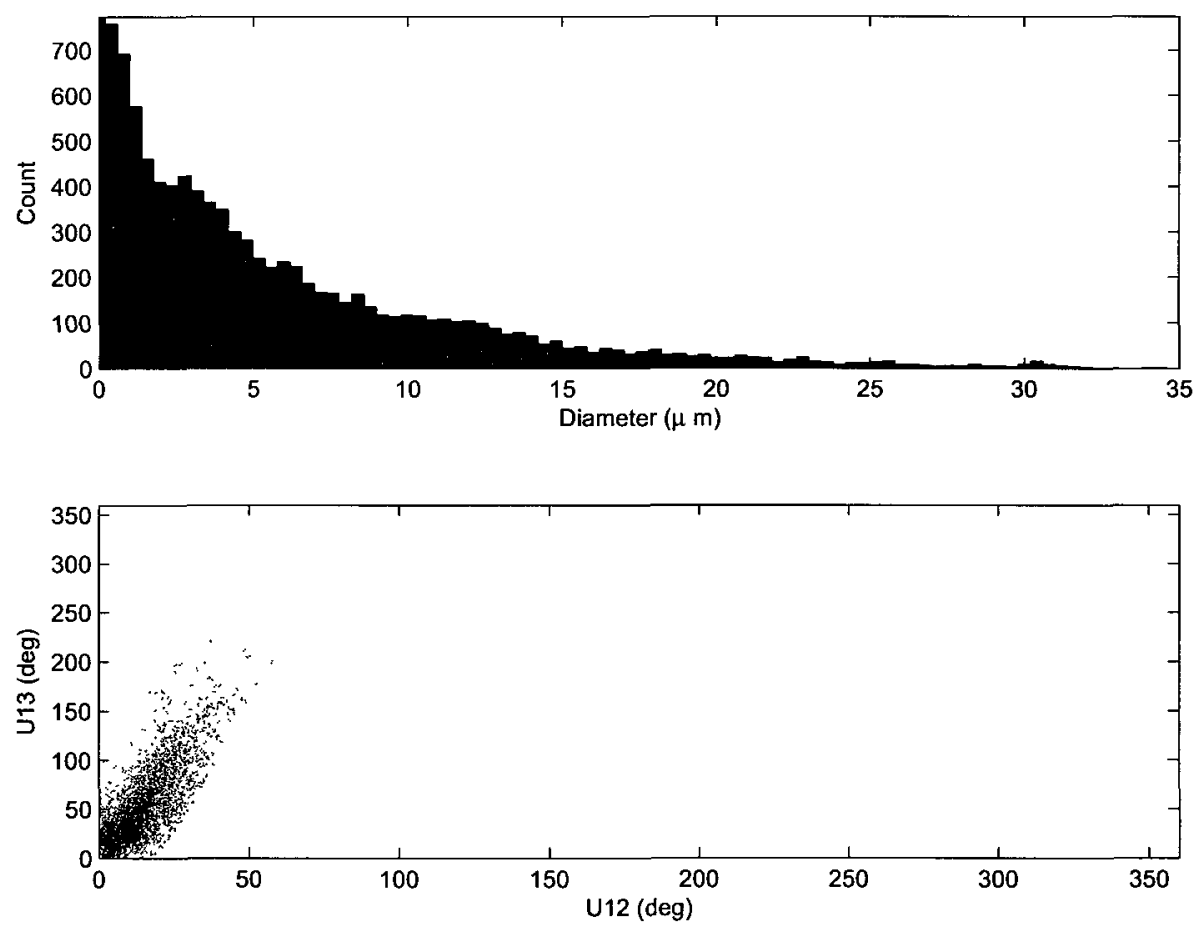

(b) Diameter histogram and PDA detector phase validation

Figure B.21: $x=70 \mathrm{~mm}, r=7.5 \mathrm{~mm}$ 

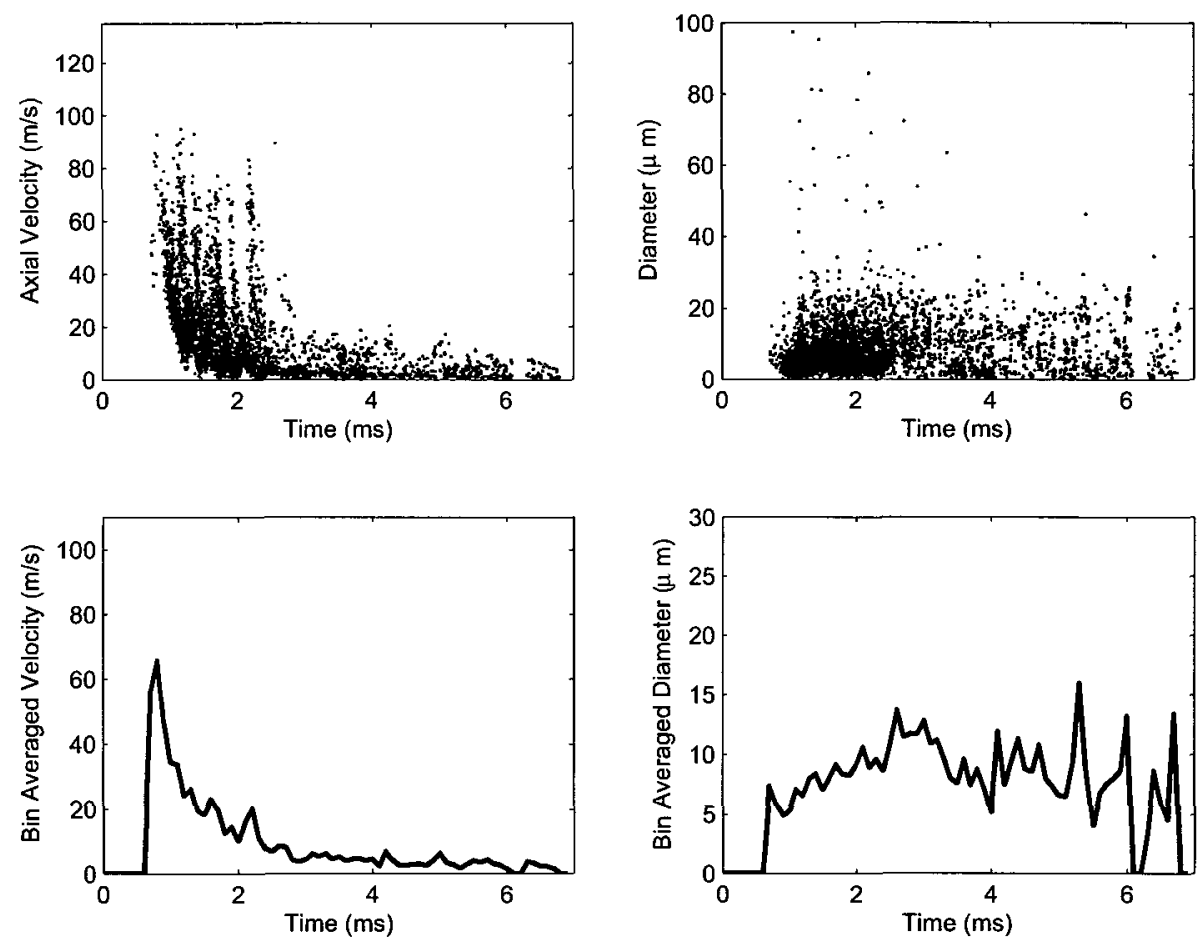

(a) Axial velocity and diameter vs time
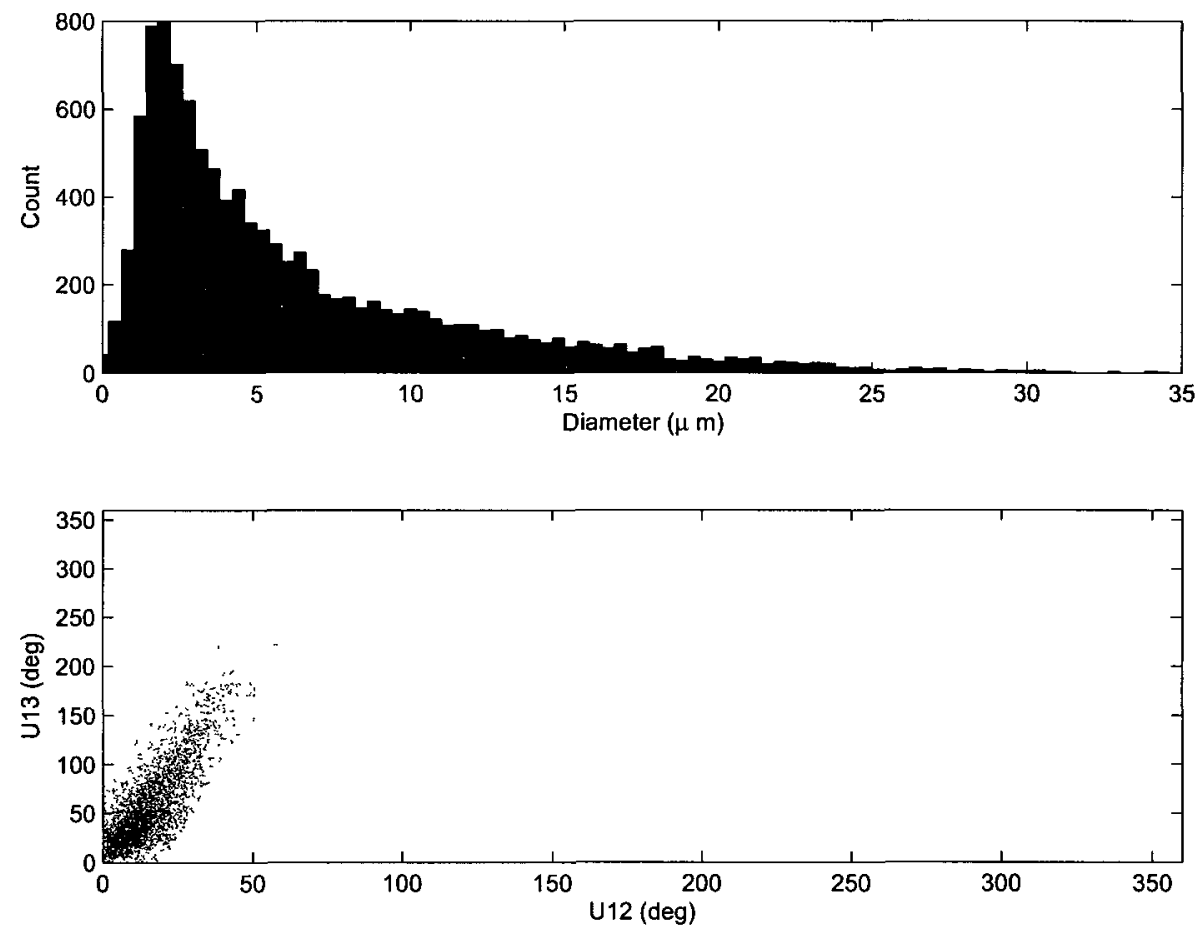

(b) Diameter histogram and PDA detector phase validation

Figure B.22: $\mathrm{x}=70 \mathrm{~mm}, \mathrm{r}=8.5 \mathrm{~mm}$ 

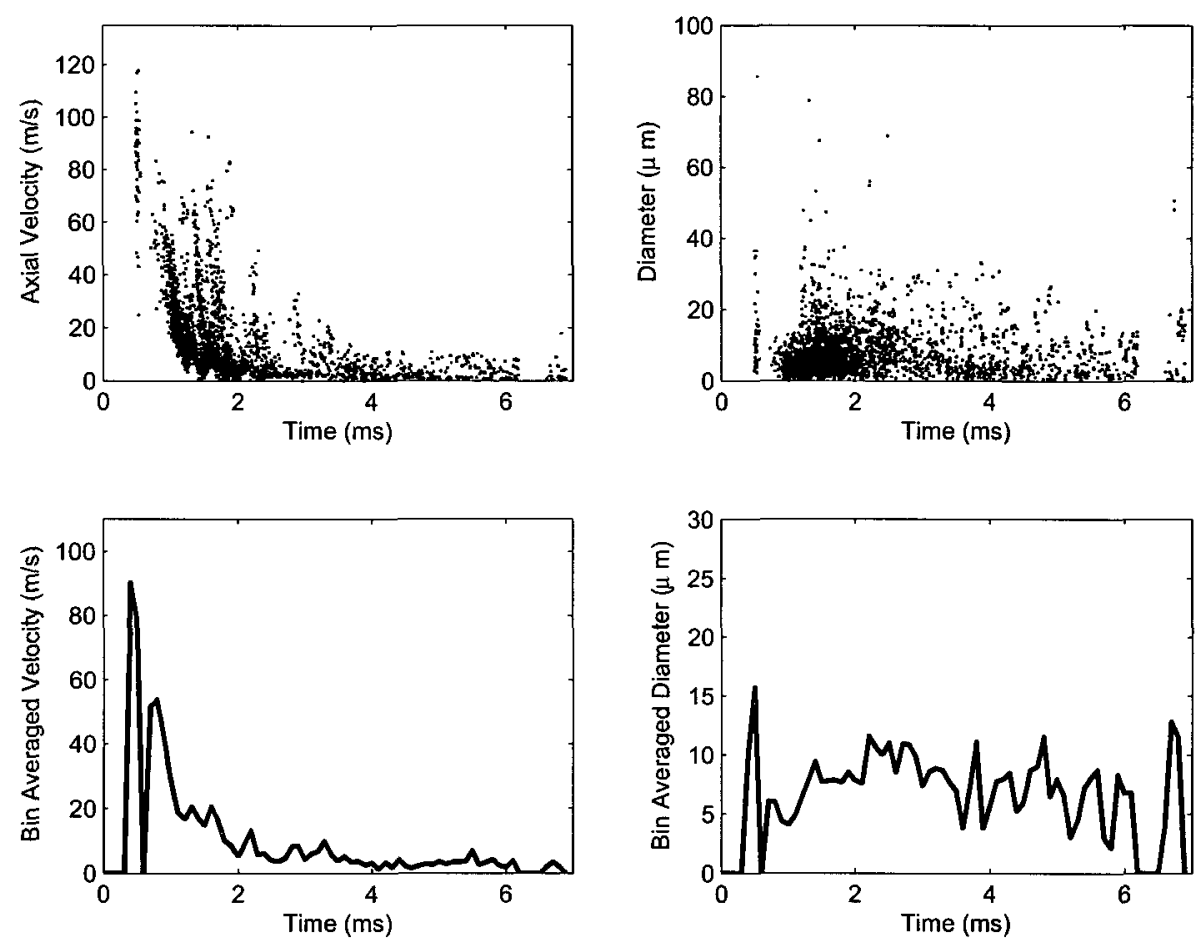

(a) Axial velocity and diameter vs time
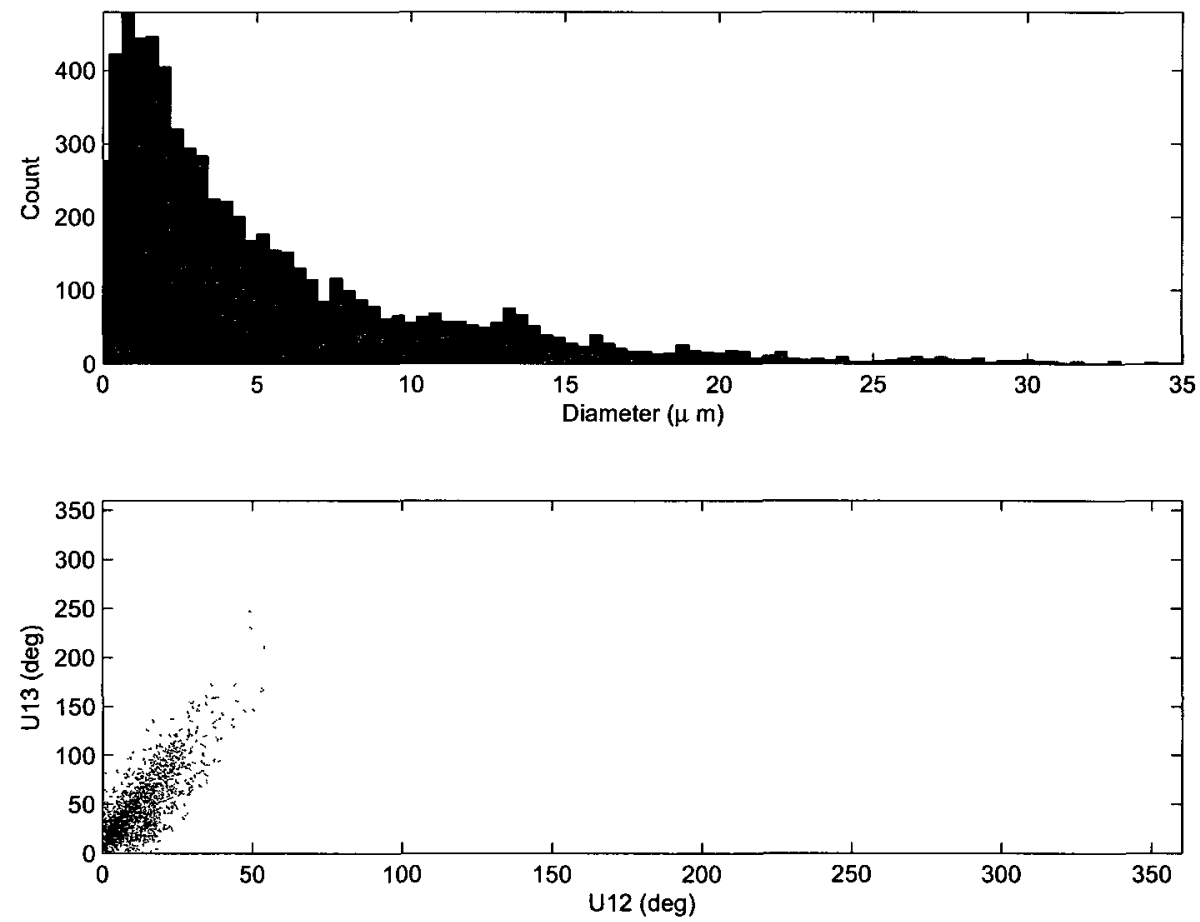

(b) Diameter histogram and PDA detector phase validation

Figure B.23: $x=70 \mathrm{~mm}, \mathrm{r}=9.5 \mathrm{~mm}$ 

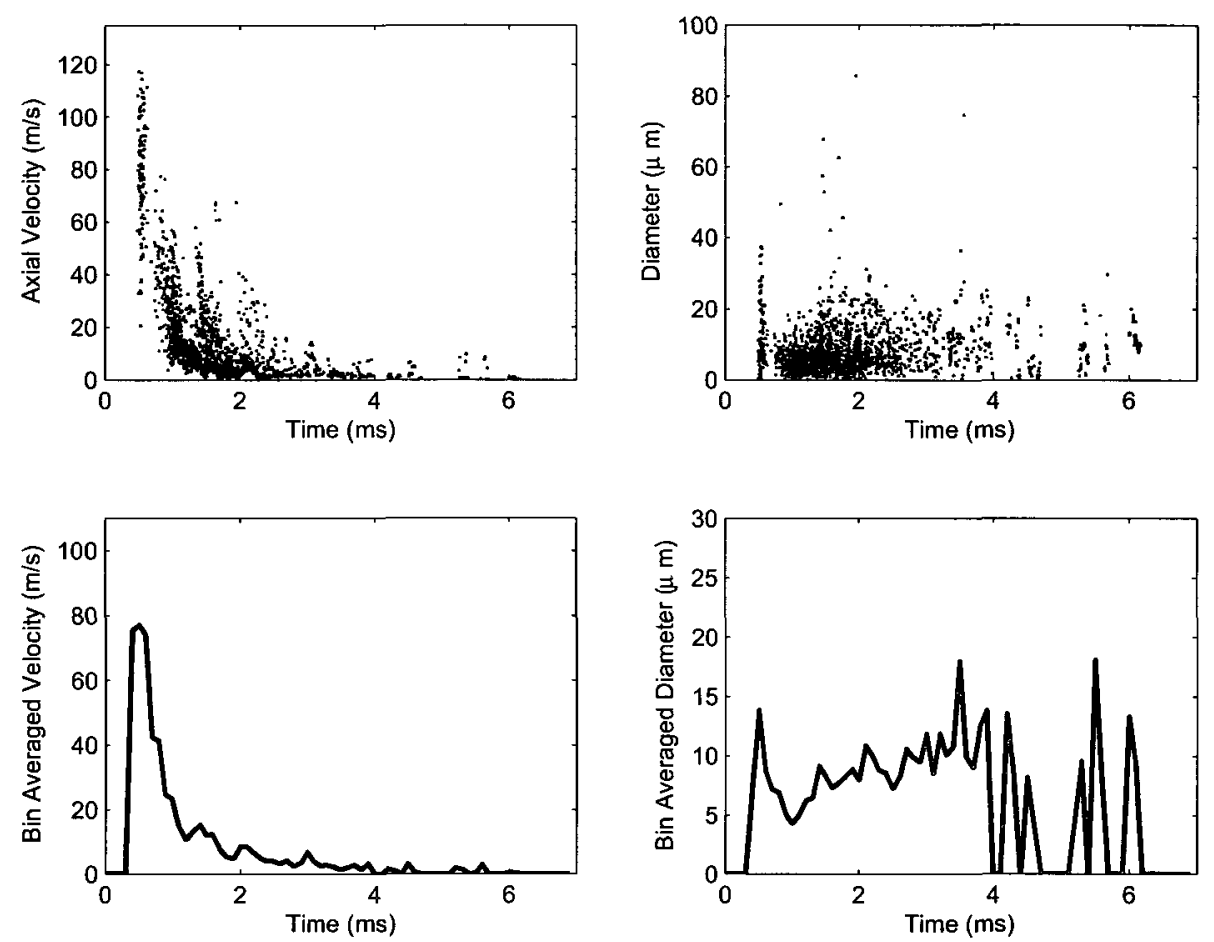

(a) Axial velocity and diameter vs time
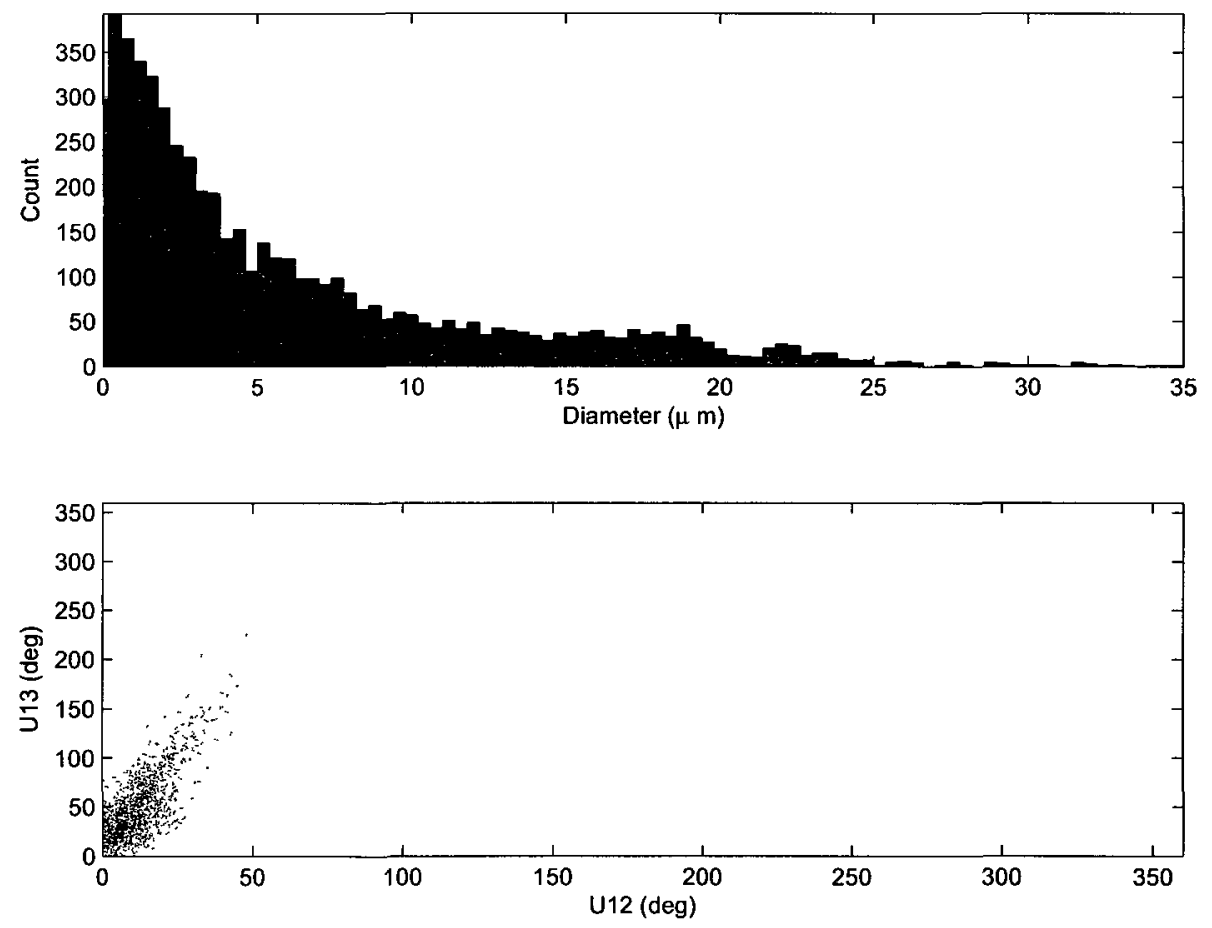

(b) Diameter histogram and PDA detector phase validation

Figure B.24: $\mathrm{x}=70 \mathrm{~mm}, \mathrm{r}=10.5 \mathrm{~mm}$ 

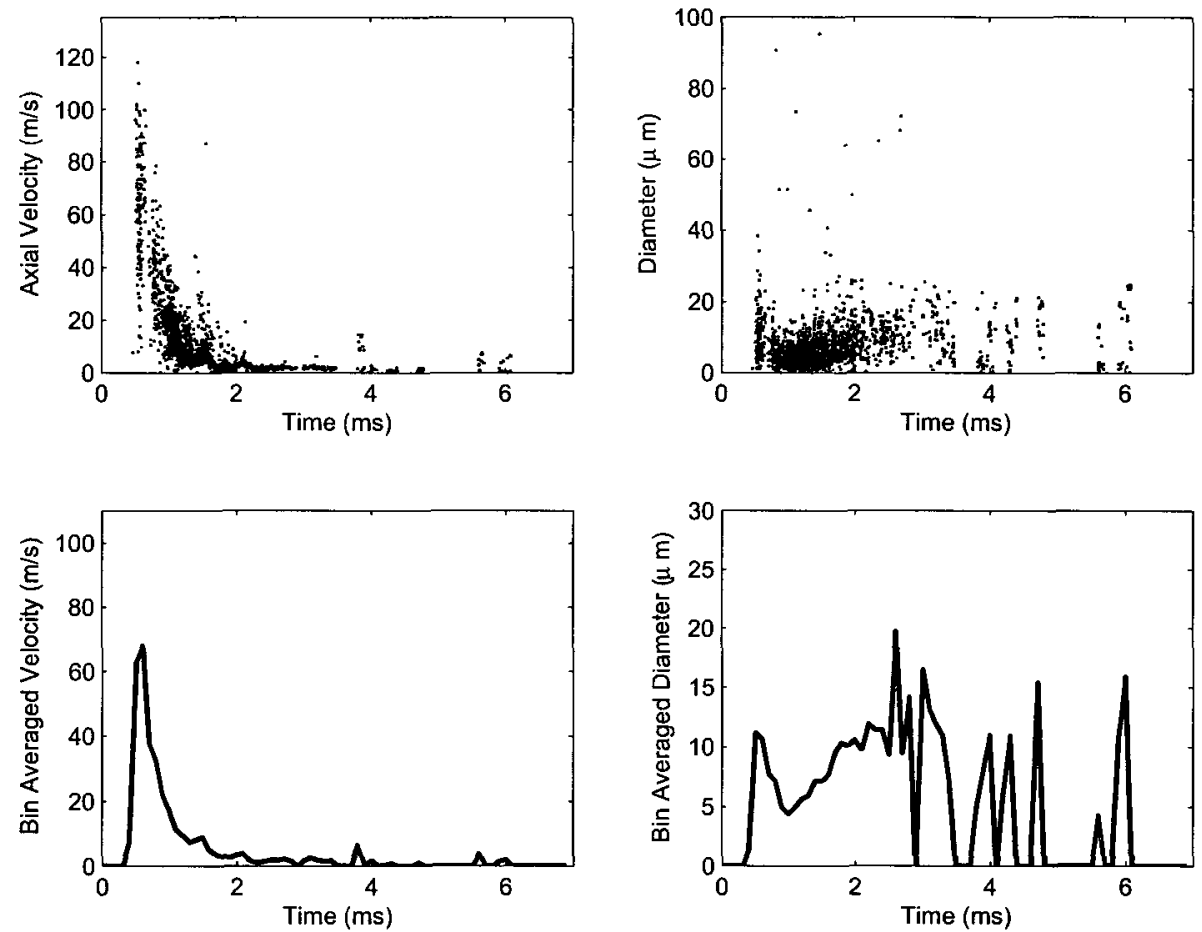

(a) Axial velocity and diameter vs time
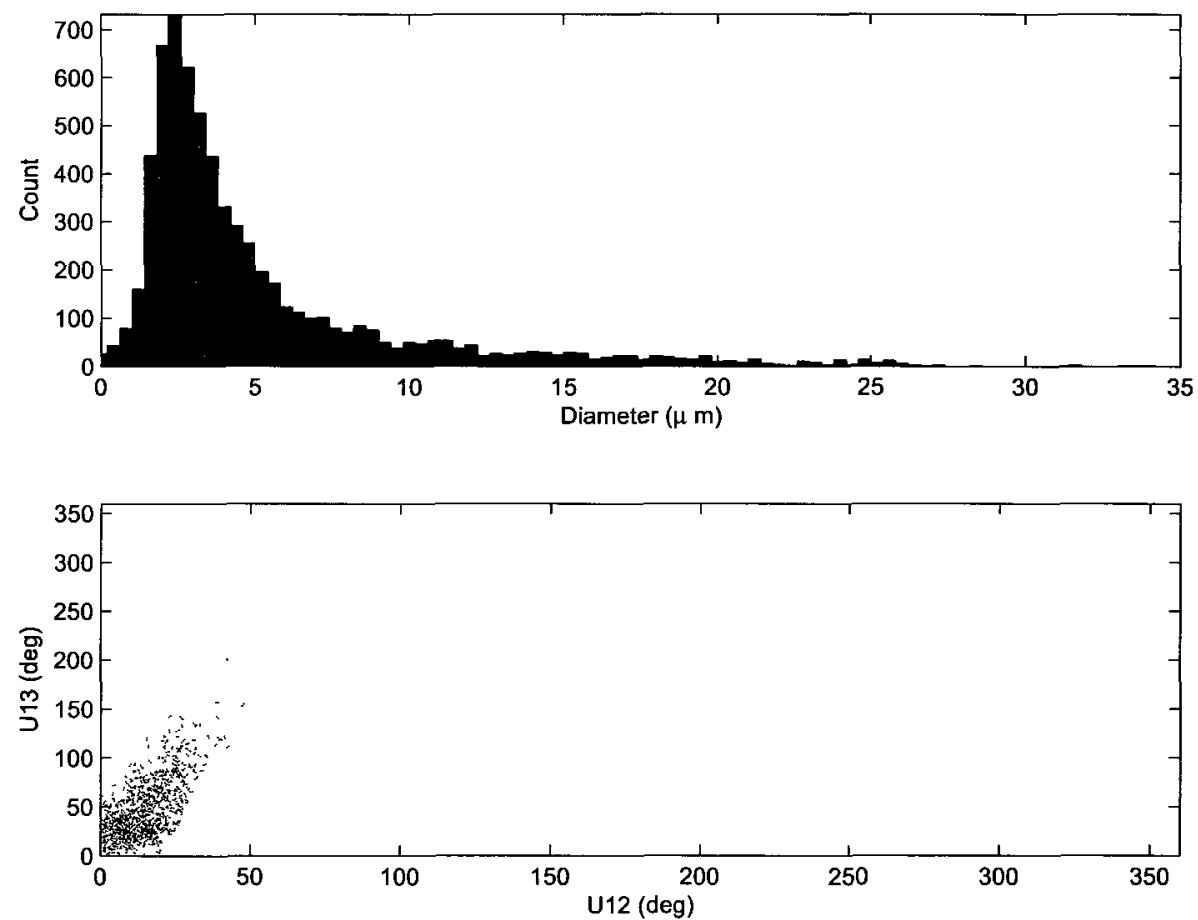

(b) Diameter histogram and PDA detector phase validation

Figure B.25: $\mathrm{x}=70 \mathrm{~mm}, \mathrm{r}=11.5 \mathrm{~mm}$ 

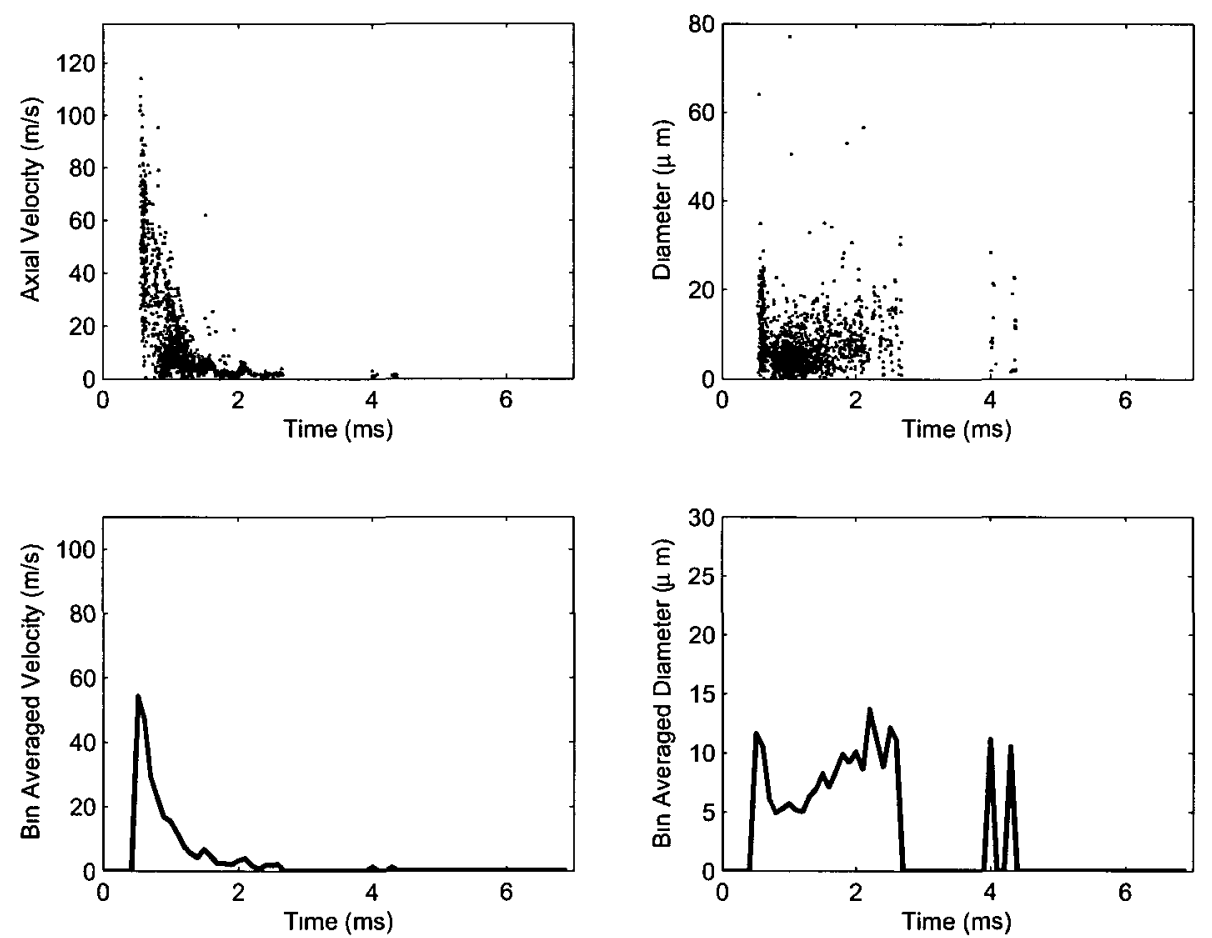

(a) Axial velocity and diameter vs time
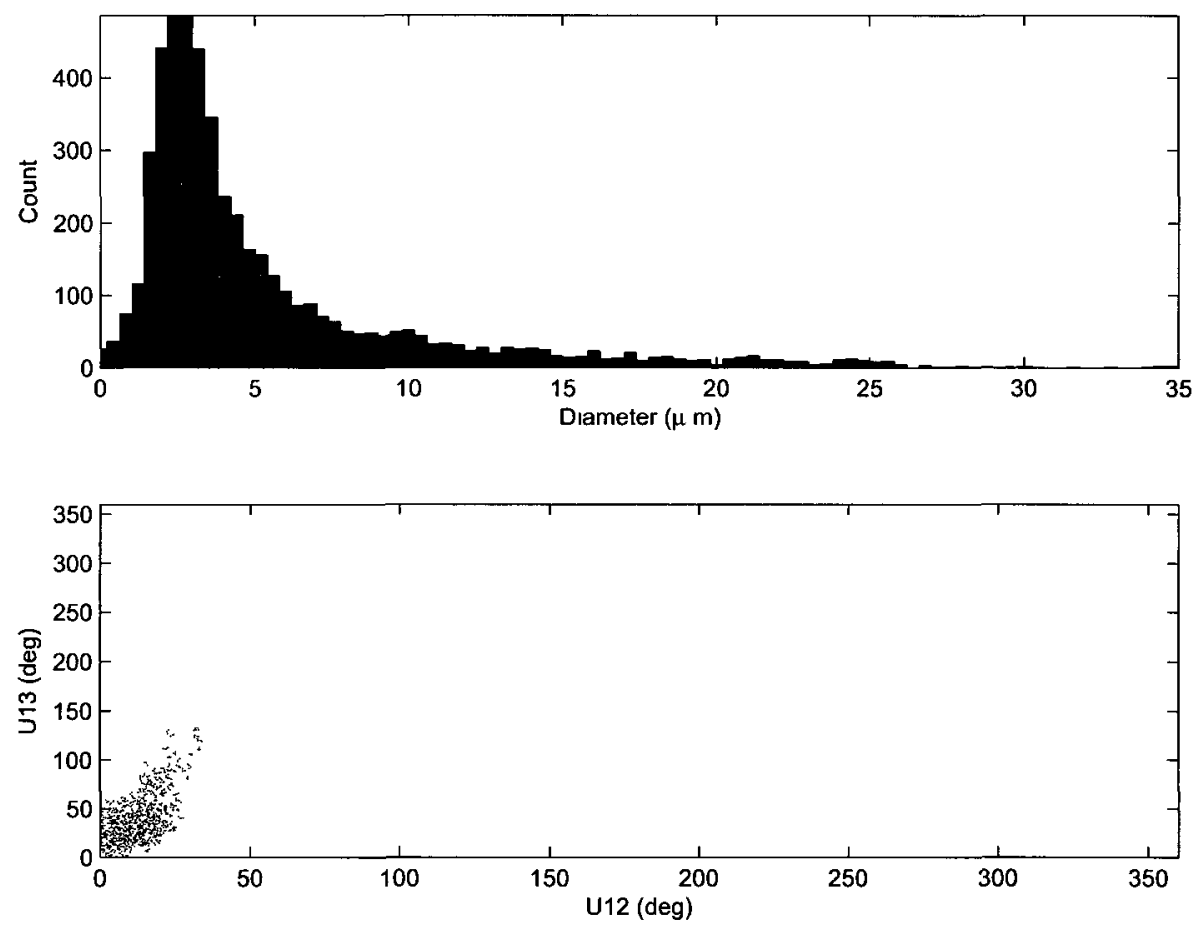

(b) Diameter histogram and PDA detector phase validation

Figure B.26: $\mathrm{x}=70 \mathrm{~mm}, \mathrm{r}=12.5 \mathrm{~mm}$ 

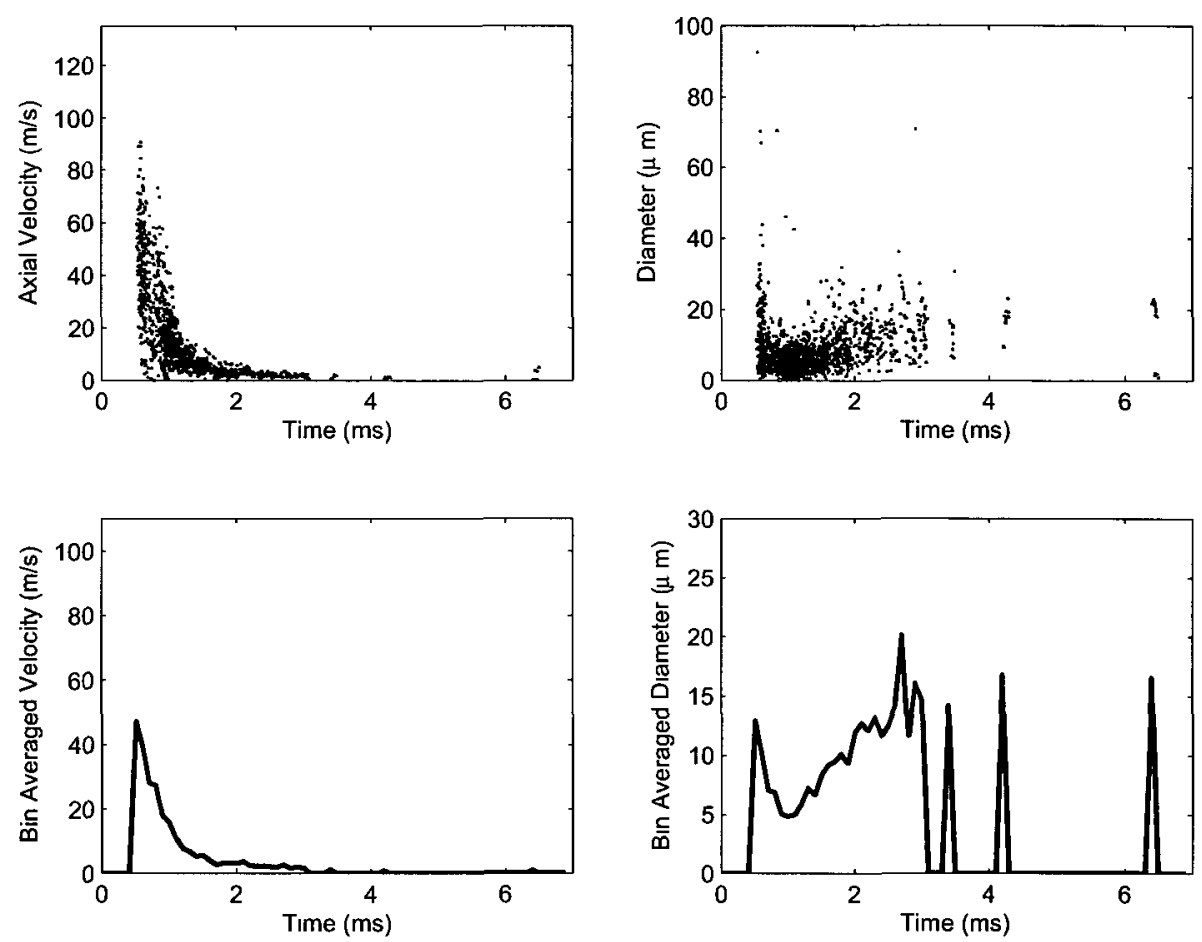

(a) Axial velocity and diameter vs time
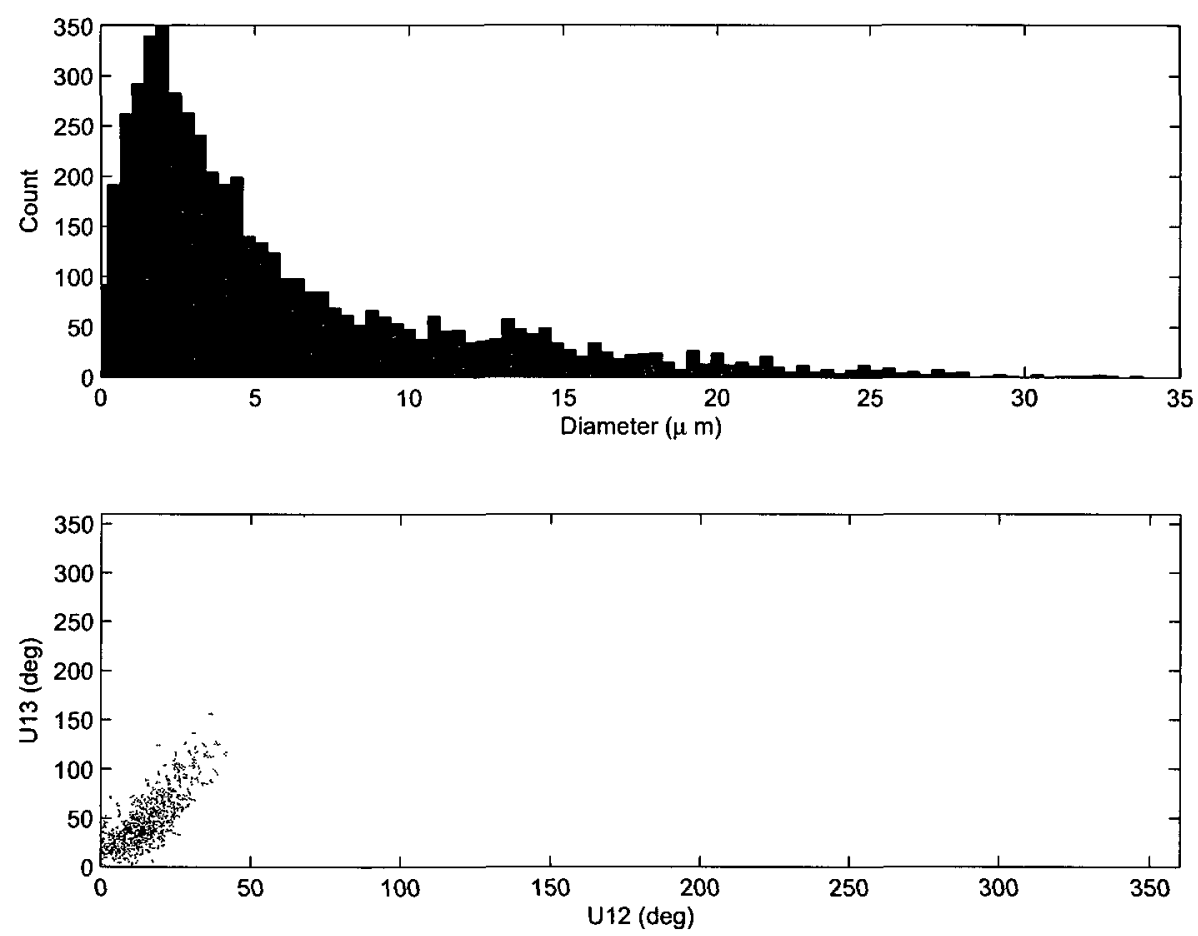

(b) Diameter histogram and PDA detector phase validation

Figure B.27: $\mathrm{x}=70 \mathrm{~mm}, \mathrm{r}=13.5 \mathrm{~mm}$ 


\section{B.1.4 $x=85 \mathrm{~mm}$}

The following pages illustrates results $85 \mathrm{~mm}$ away from the nozzle tip: 

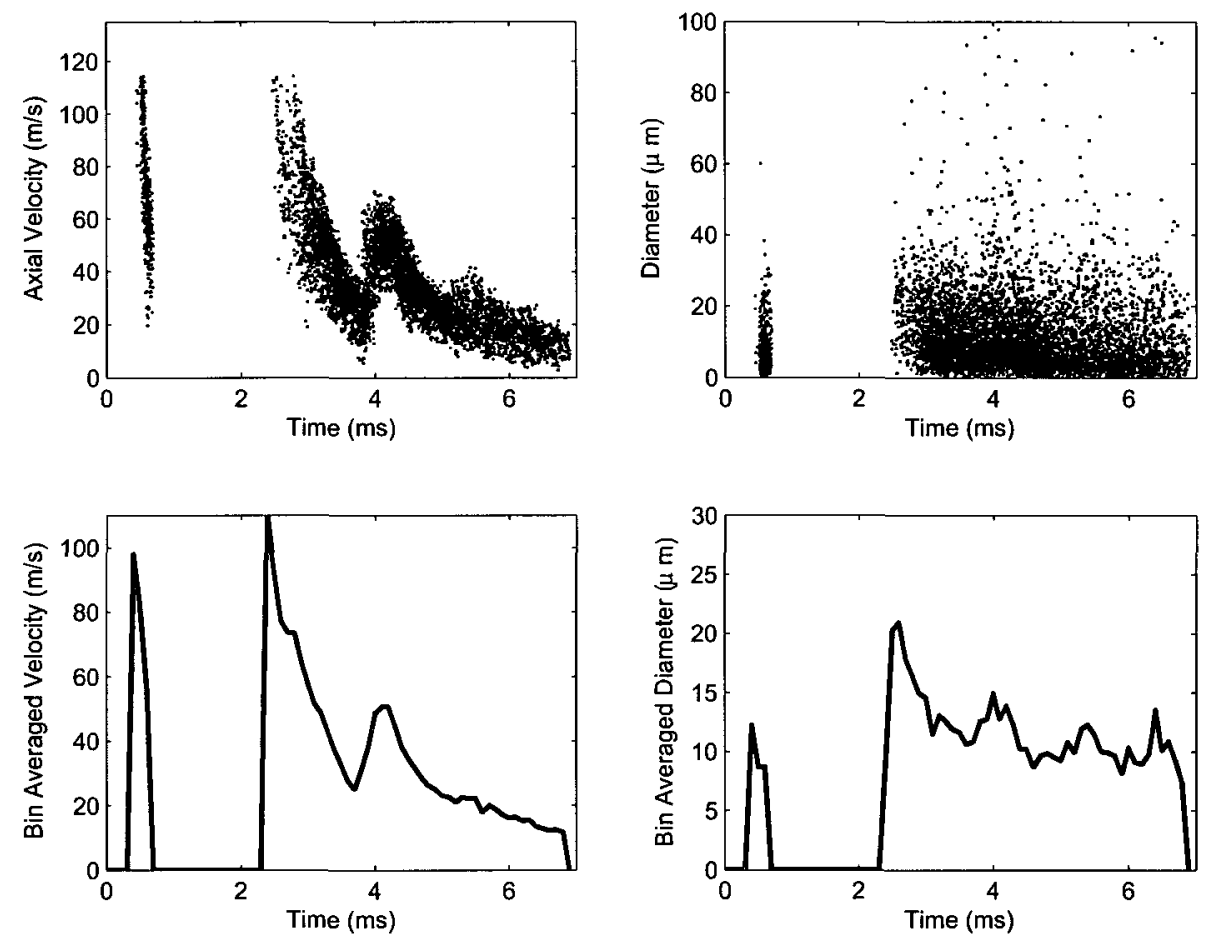

(a) Axial velocity and diameter vs time
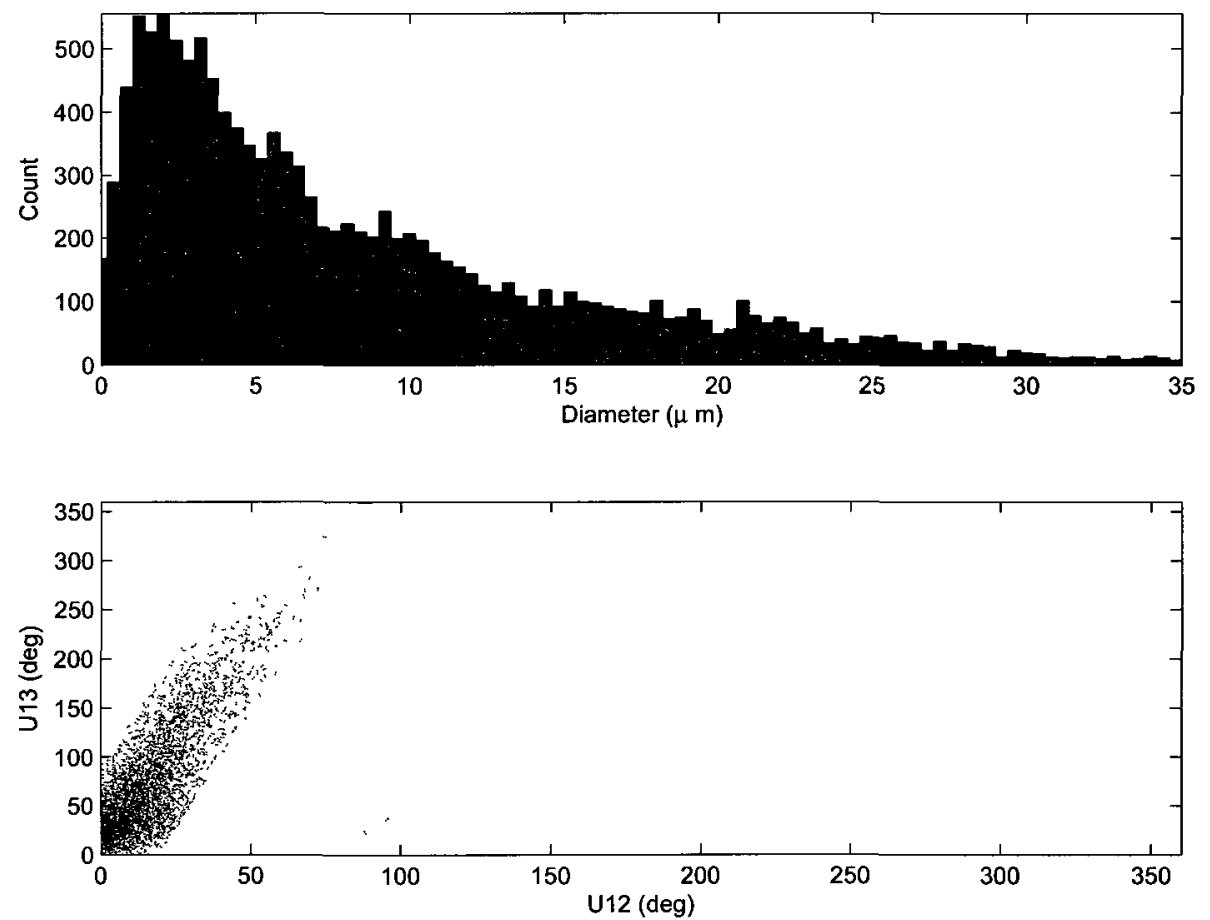

(b) Diameter histogram and PDA detector phase validation

Figure B.28: $\mathrm{x}=85 \mathrm{~mm}, \mathrm{r}=0 \mathrm{~mm}$ 

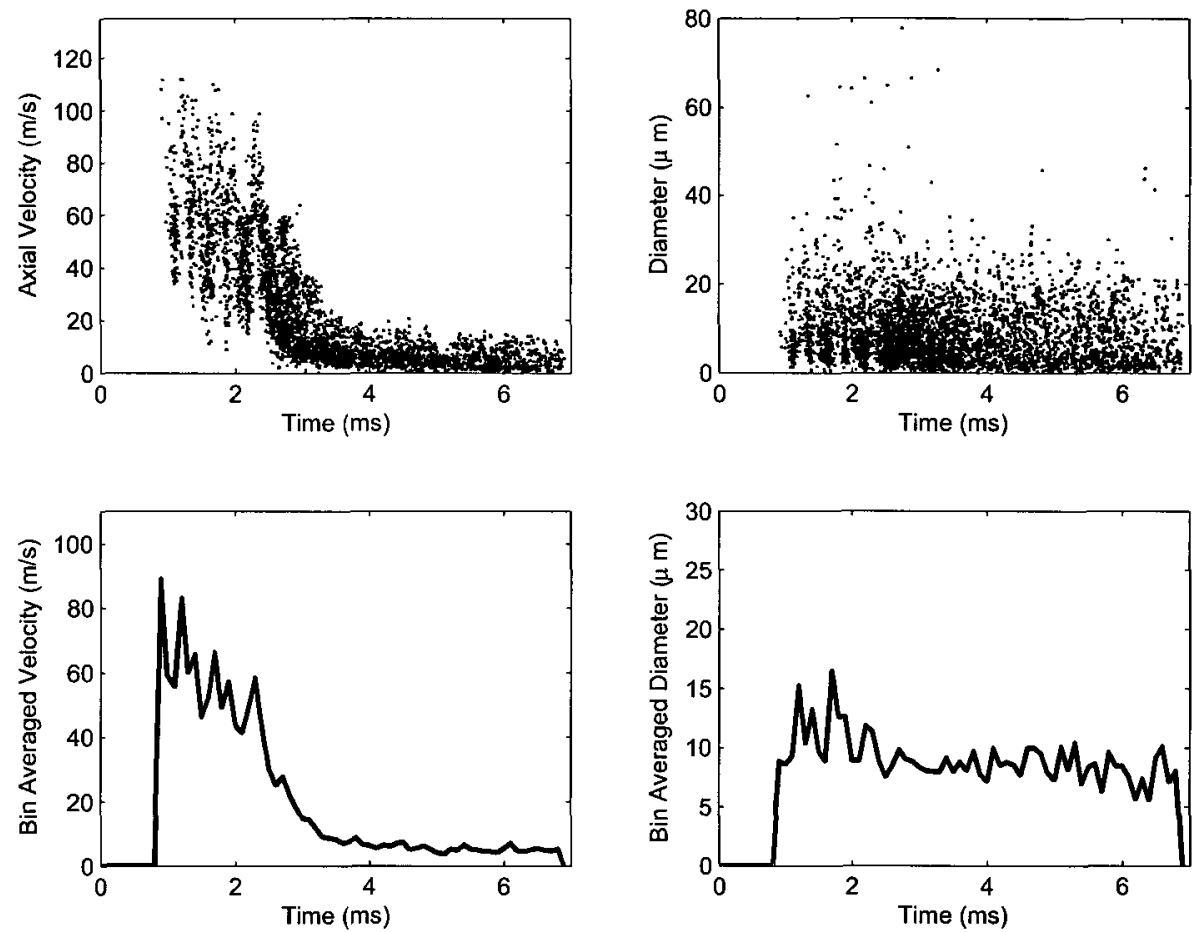

(a) Axial velocity and diameter vs time
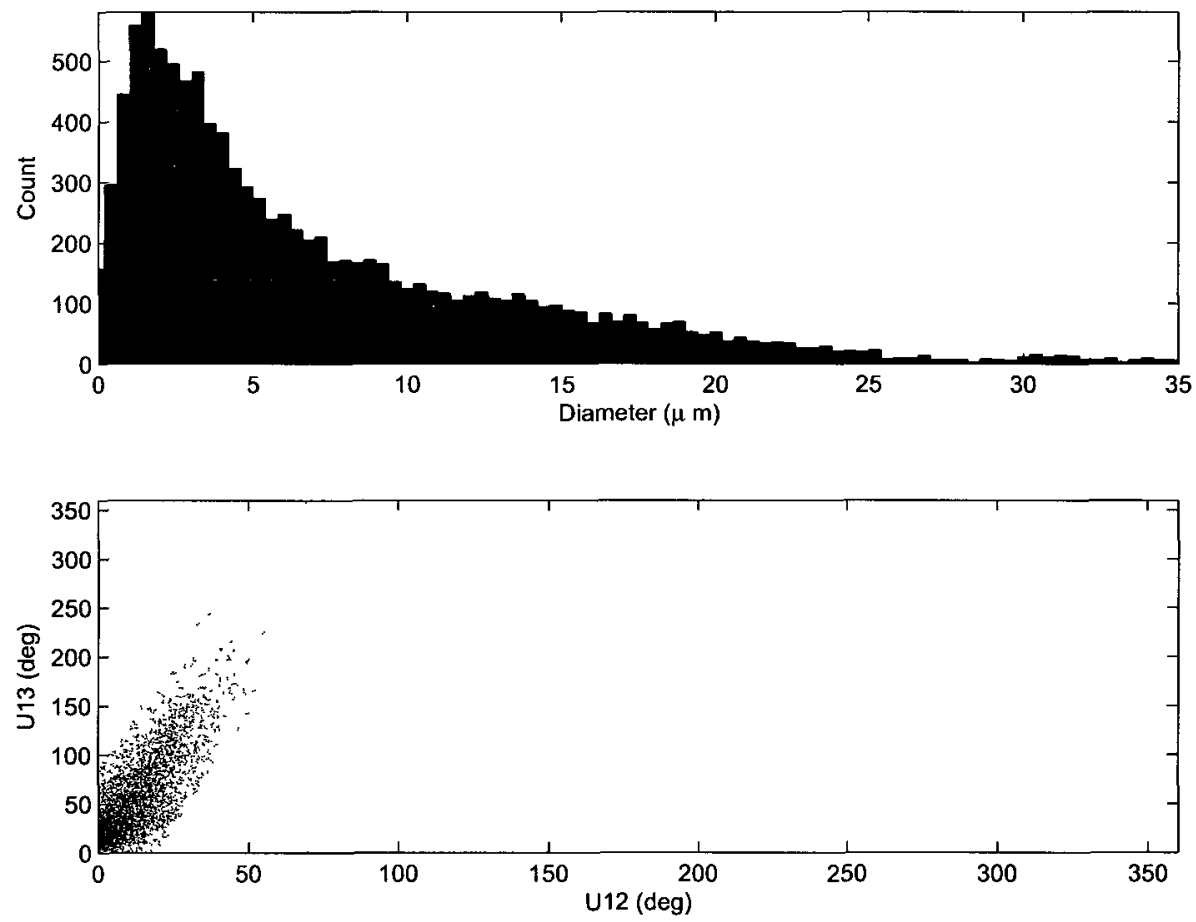

(b) Diameter histogram and PDA detector phase validation

Figure B.29: $\mathrm{x}=85 \mathrm{~mm}, \mathrm{r}=7.5 \mathrm{~mm}$ 

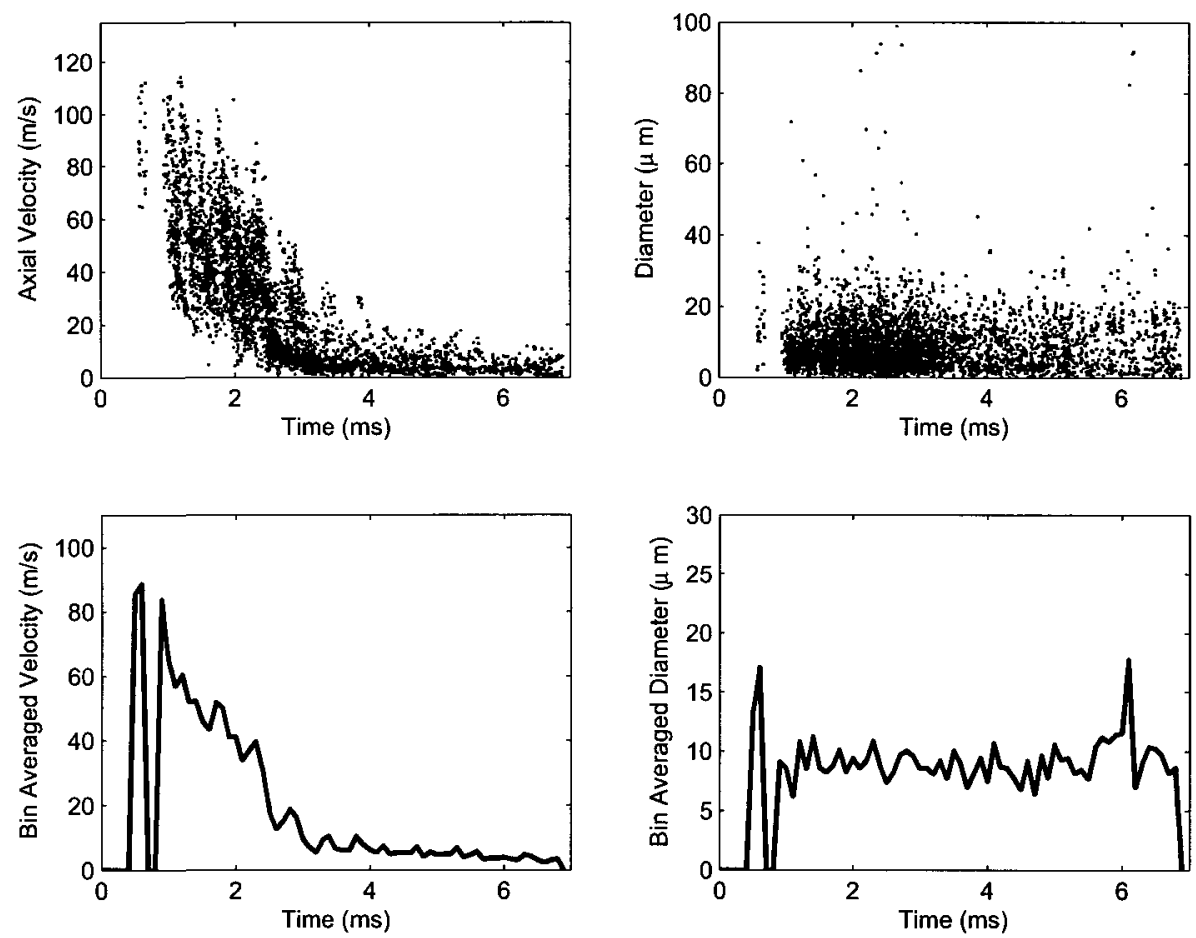

(a) Axial velocity and diameter vs time
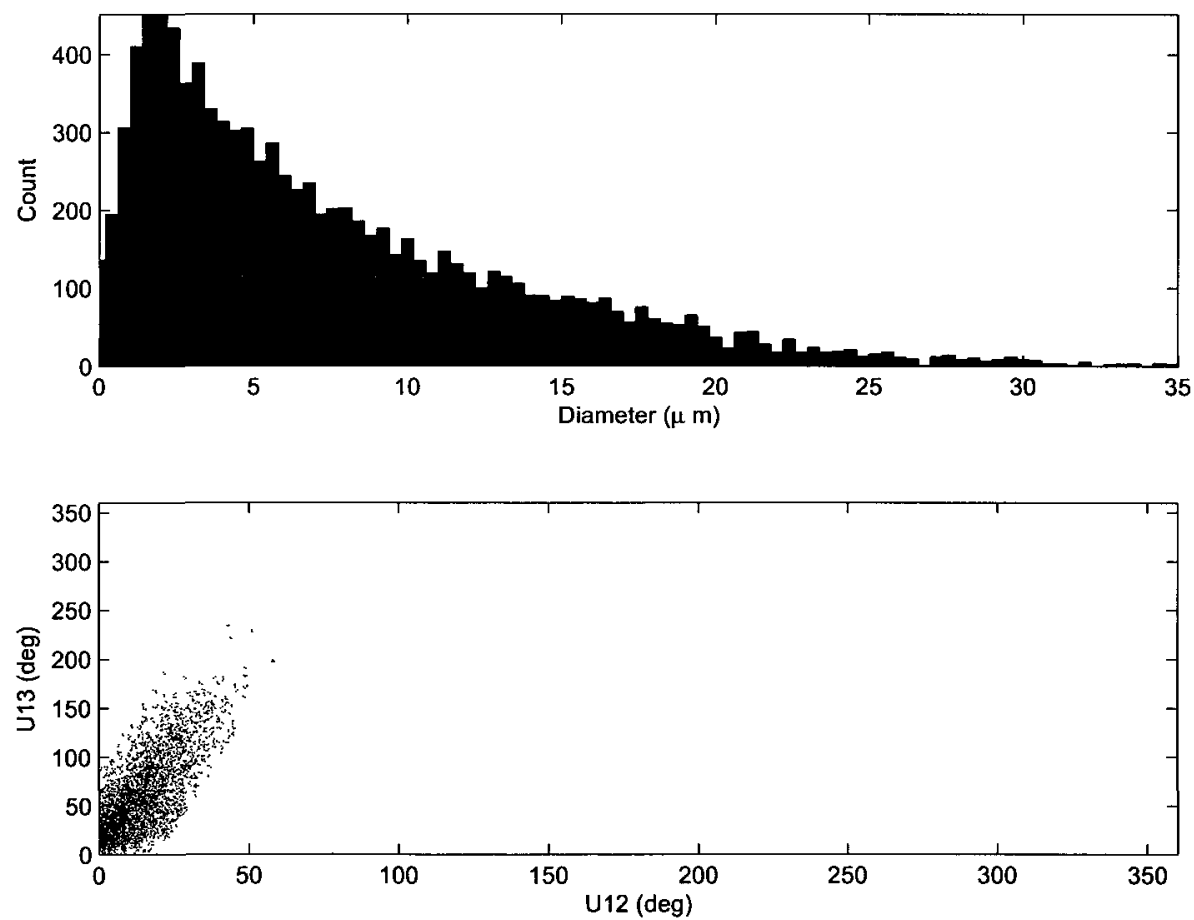

(b) Diameter histogram and PDA detector phase validation

Figure B.30: $\mathrm{x}=85 \mathrm{~mm}, \mathrm{r}=8.5 \mathrm{~mm}$ 

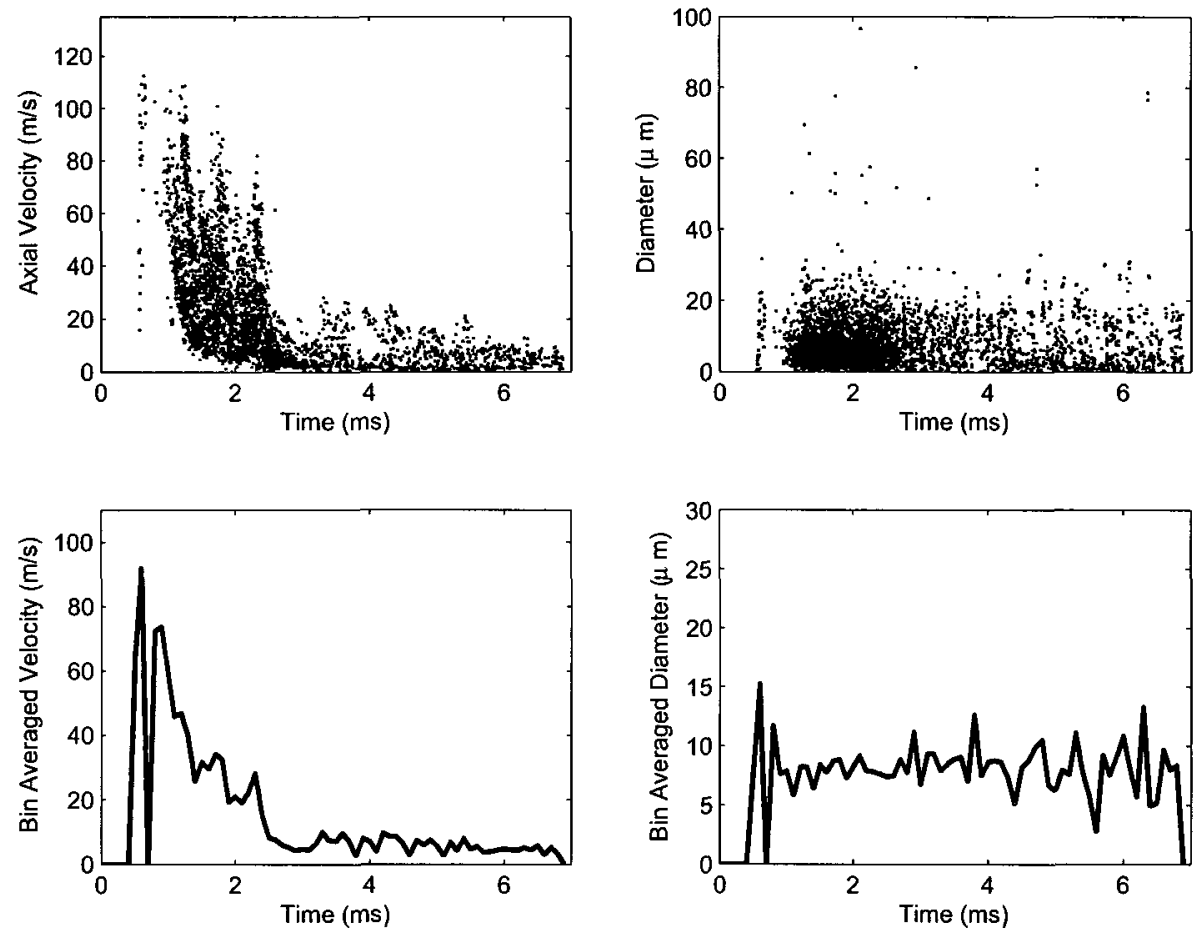

(a) Axial velocity and diameter vs time
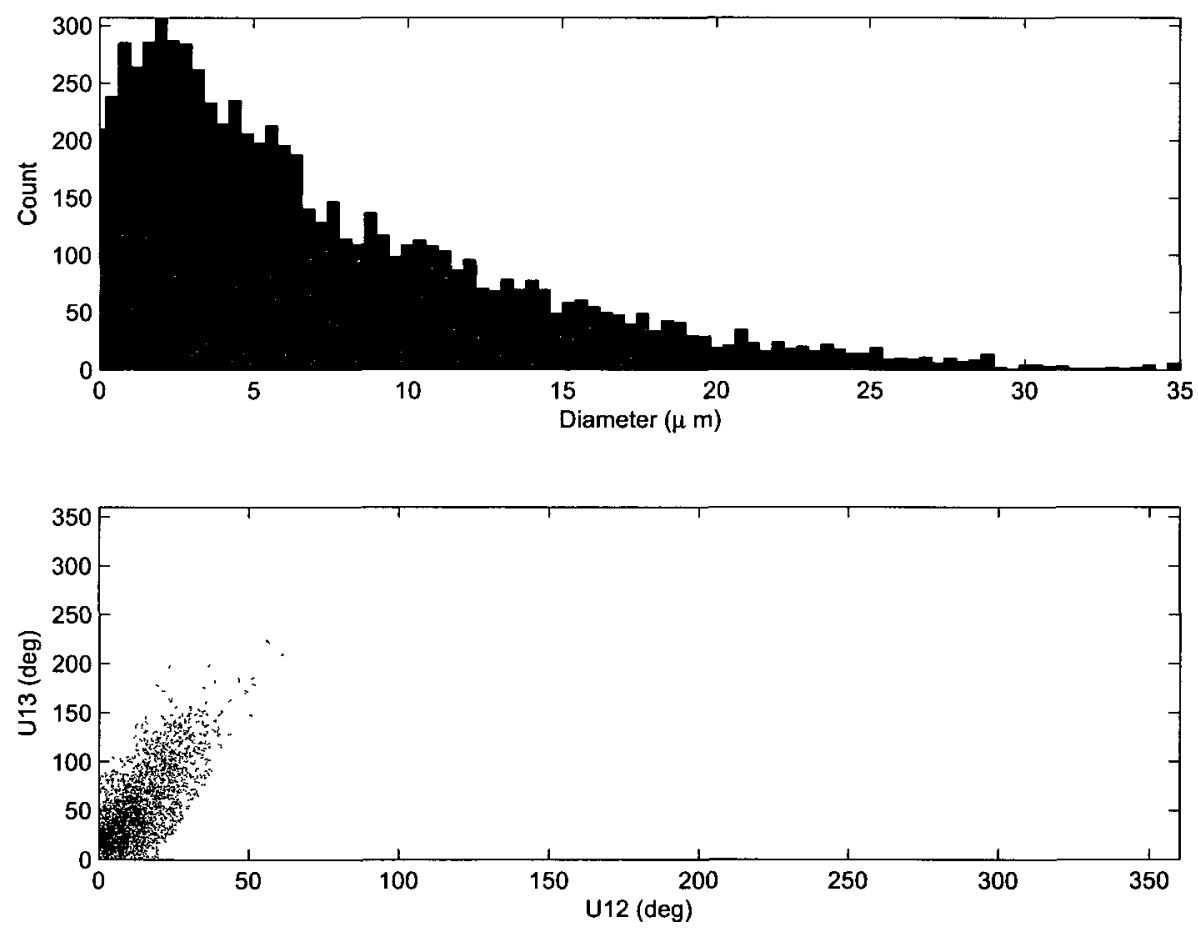

(b) Diameter histogram and PDA detector phase validation

Figure B.31: $\mathrm{x}=85 \mathrm{~mm}, \mathrm{r}=9.5 \mathrm{~mm}$ 

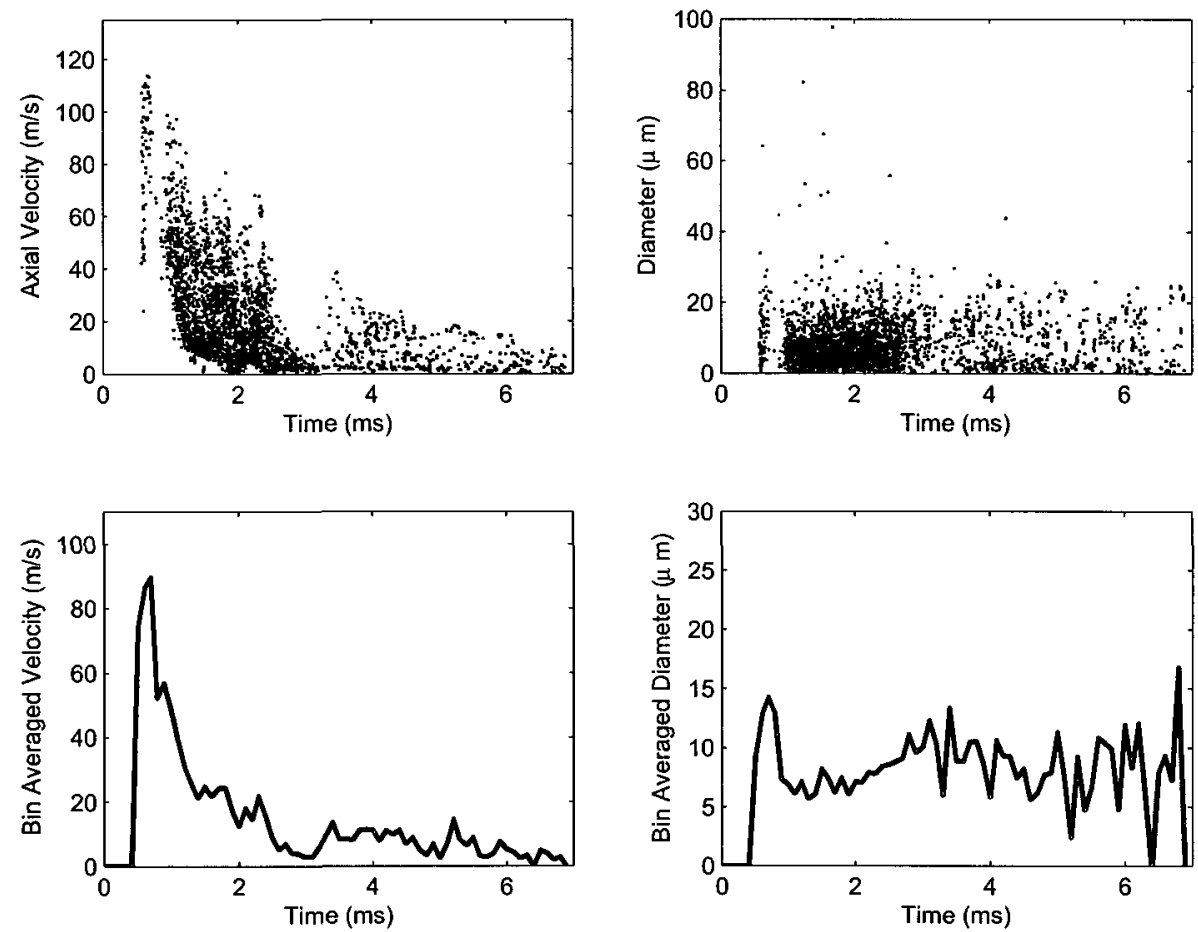

(a) Axial velocity and diameter vs time
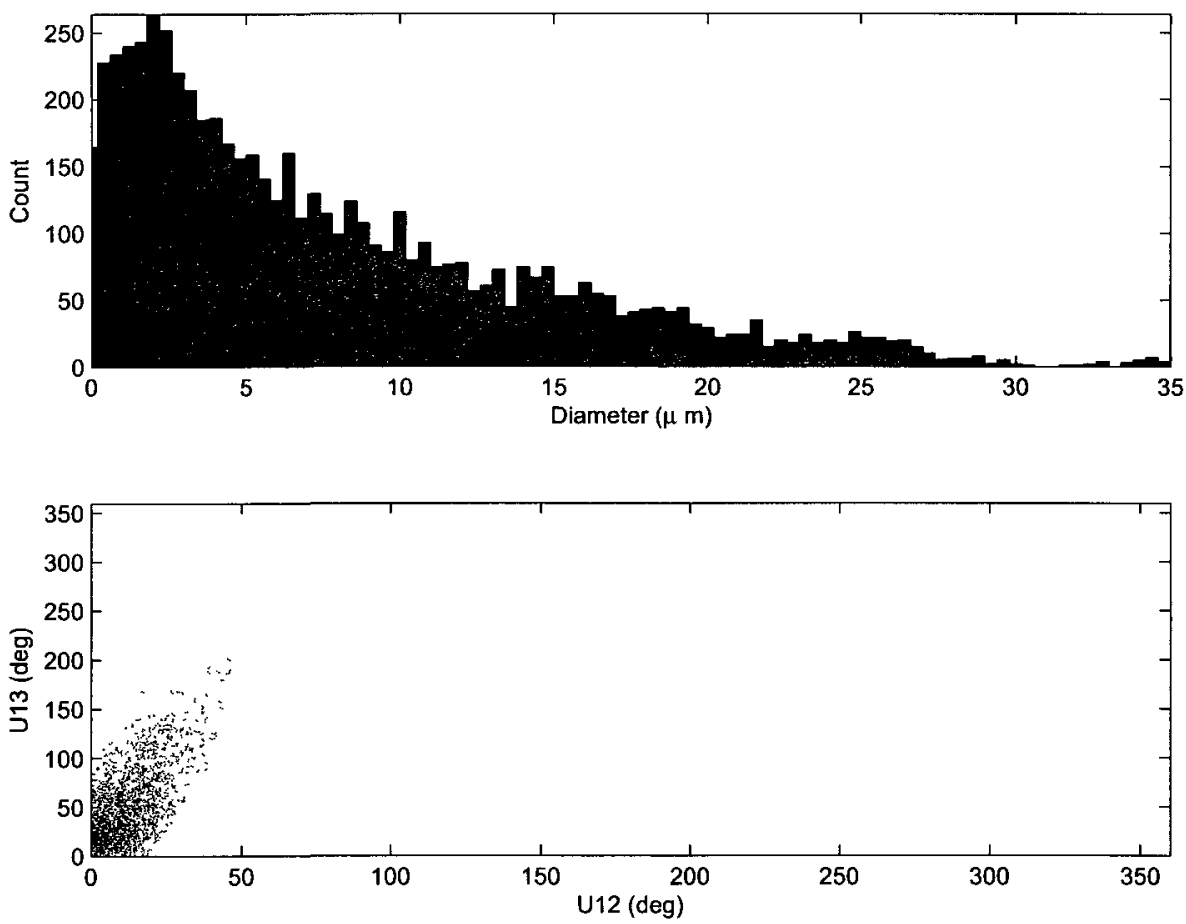

(b) Diameter histogram and PDA detector phase validation

Figure B.32: $\mathrm{x}=85 \mathrm{~mm}, \mathrm{r}=10.5 \mathrm{~mm}$ 

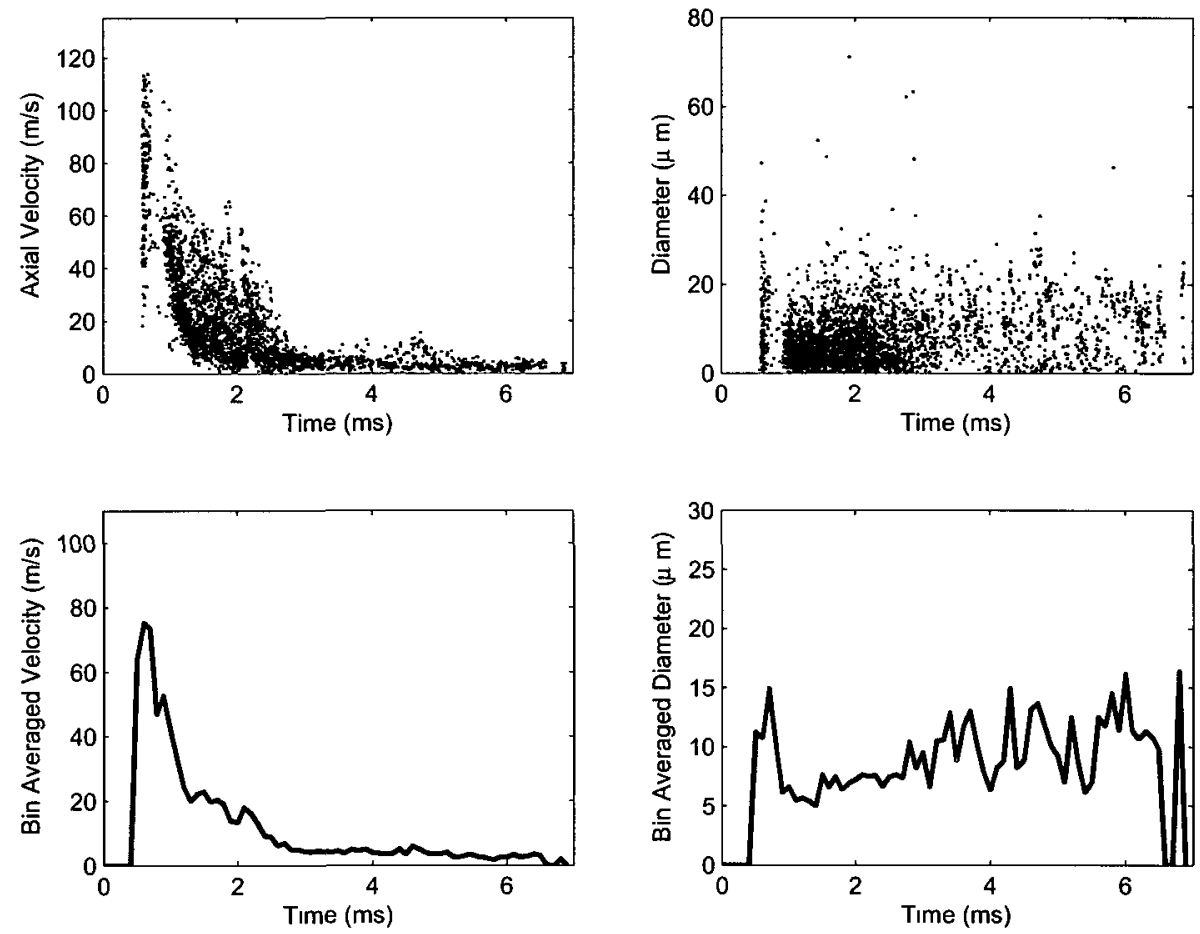

(a) Axial velocity and diameter vs time
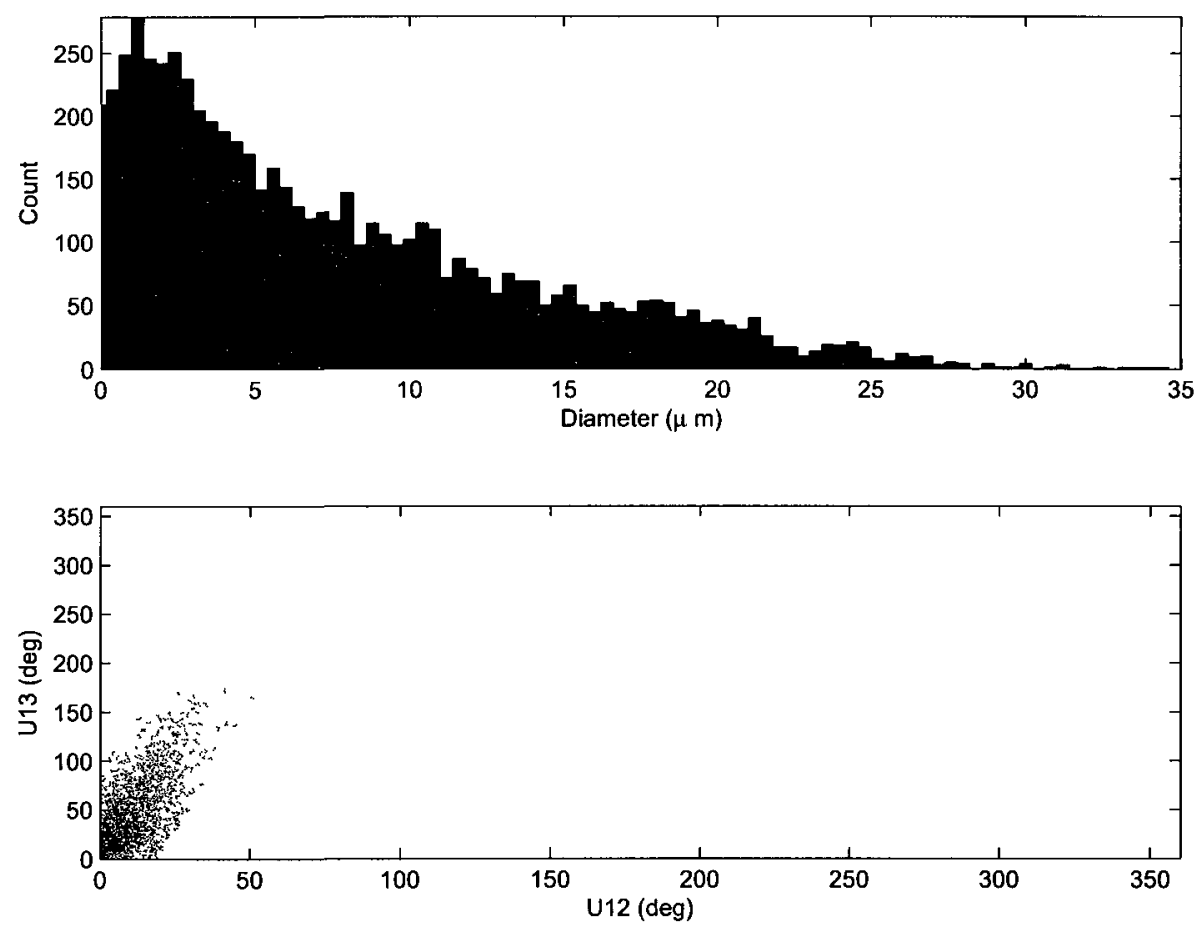

(b) Diameter histogram and PDA detector phase validation

Figure B.33: $x=85 \mathrm{~mm}, \mathrm{r}=11.5 \mathrm{~mm}$ 

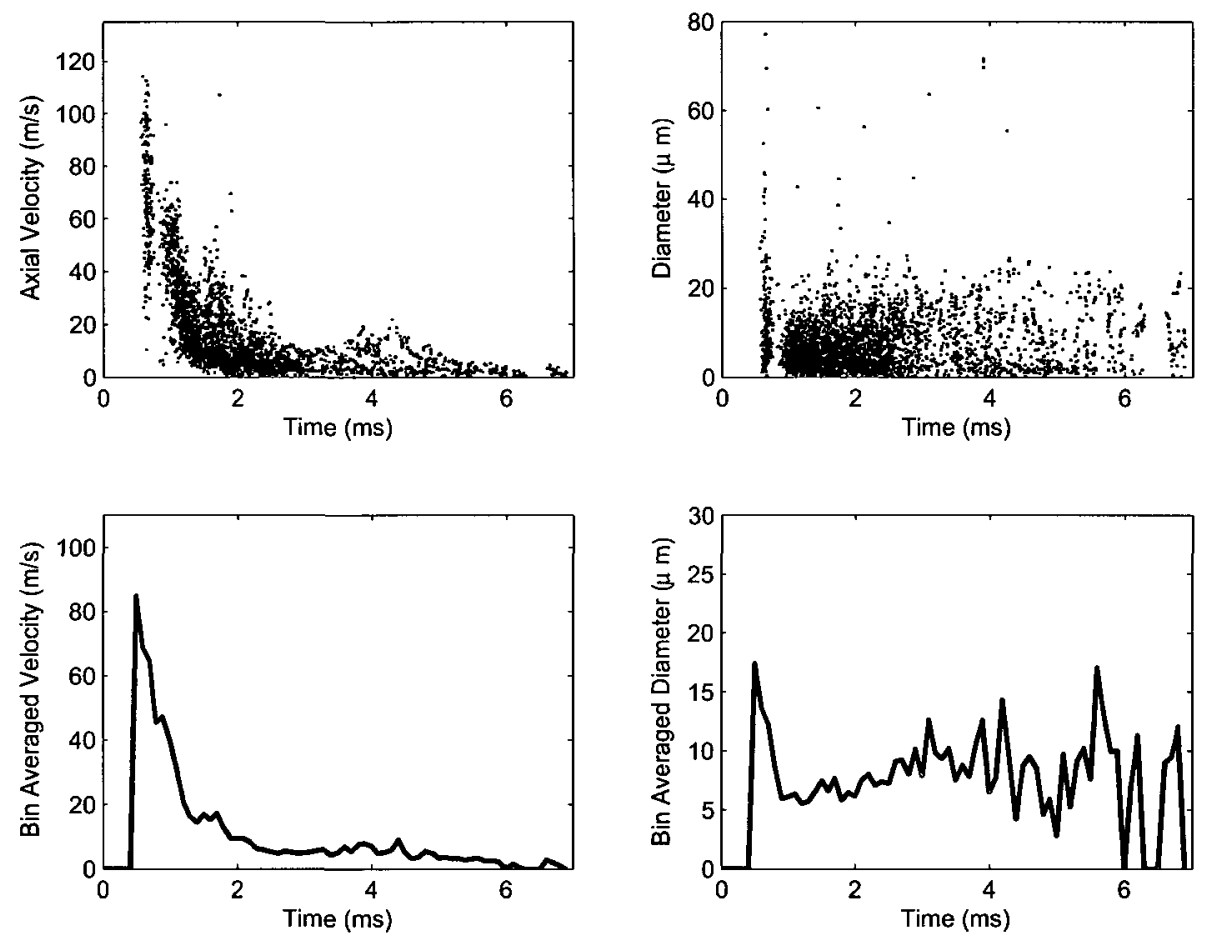

(a) Axial velocity and diameter vs time
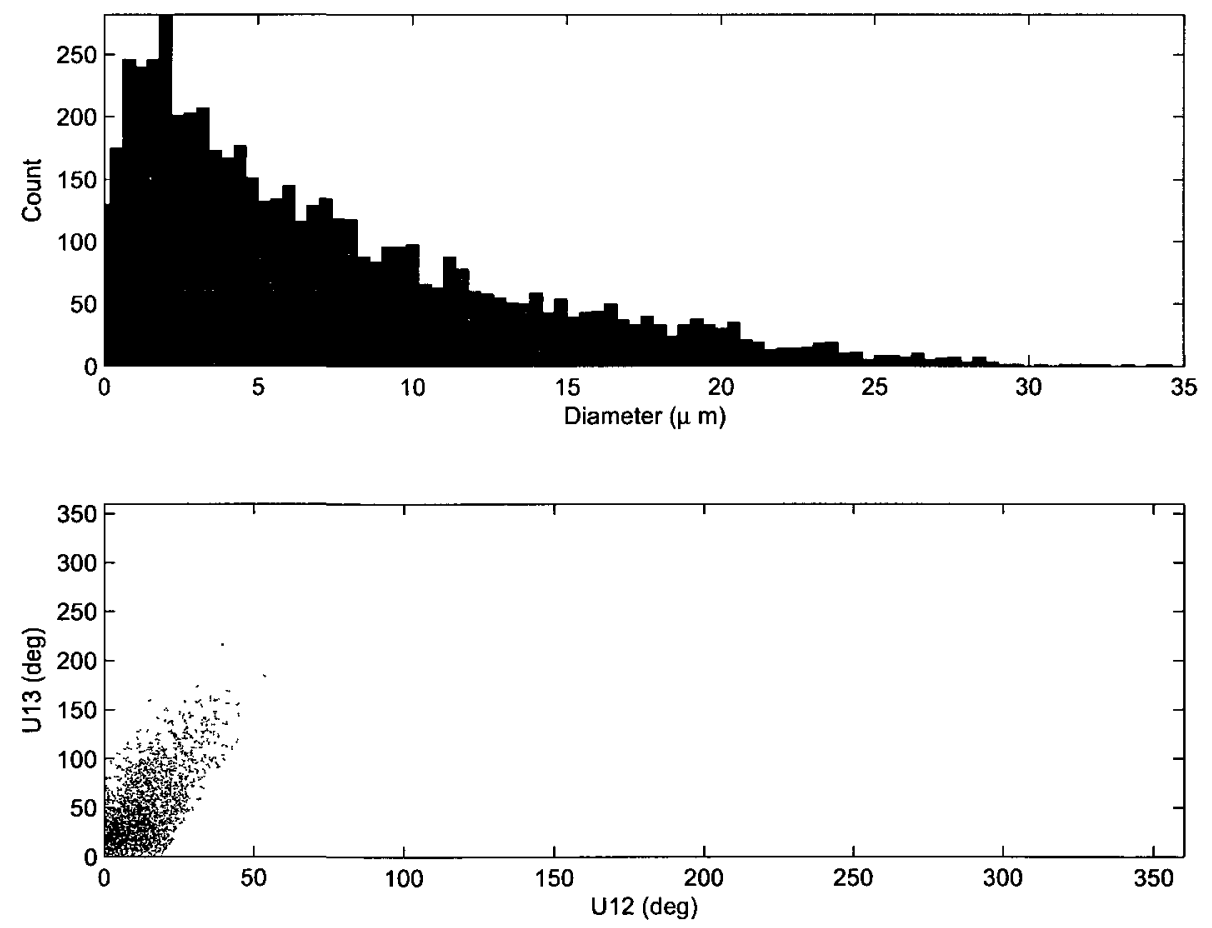

(b) Diameter histogram and PDA detector phase validation

Figure B.34: $\mathrm{x}=85 \mathrm{~mm}, \mathrm{r}=12.5 \mathrm{~mm}$ 

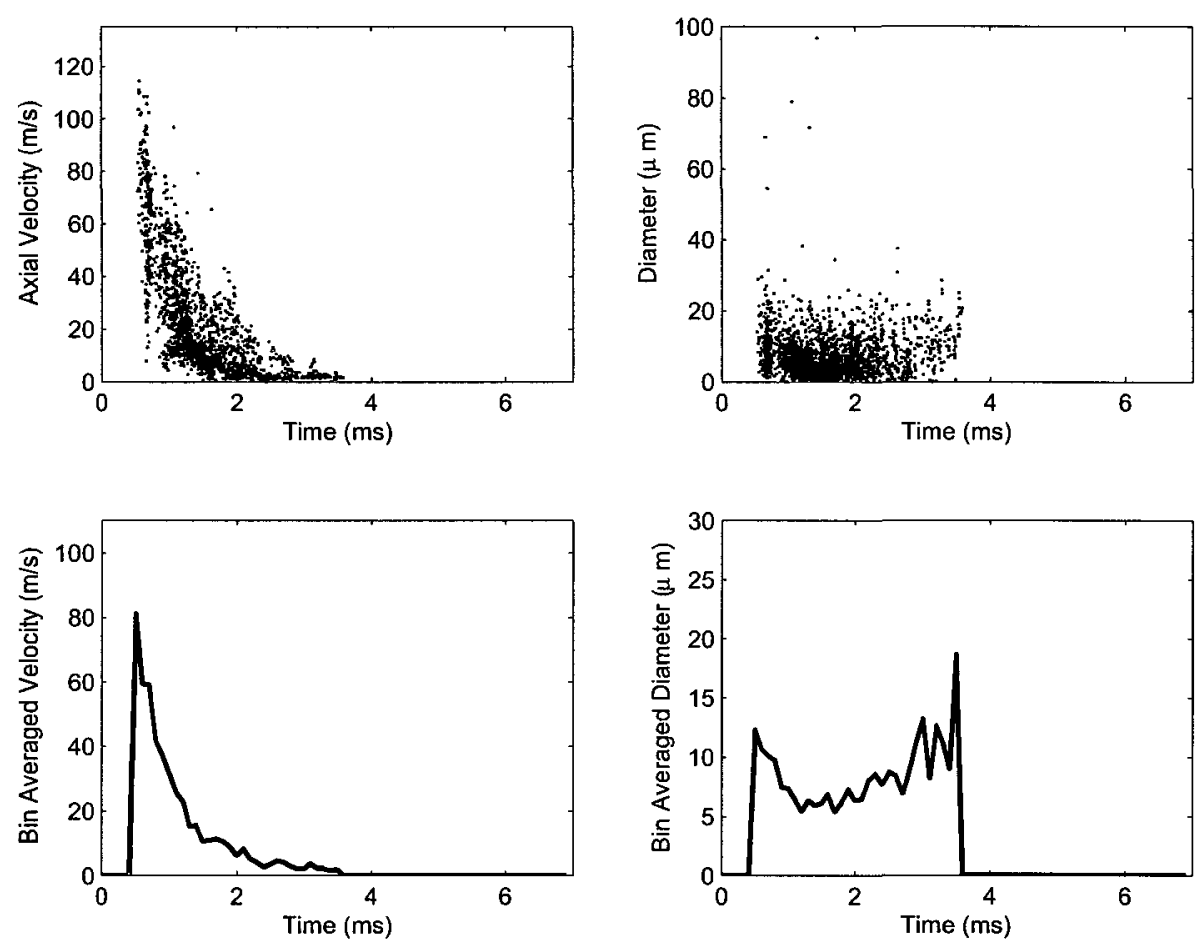

(a) Axial velocity and diameter vs time
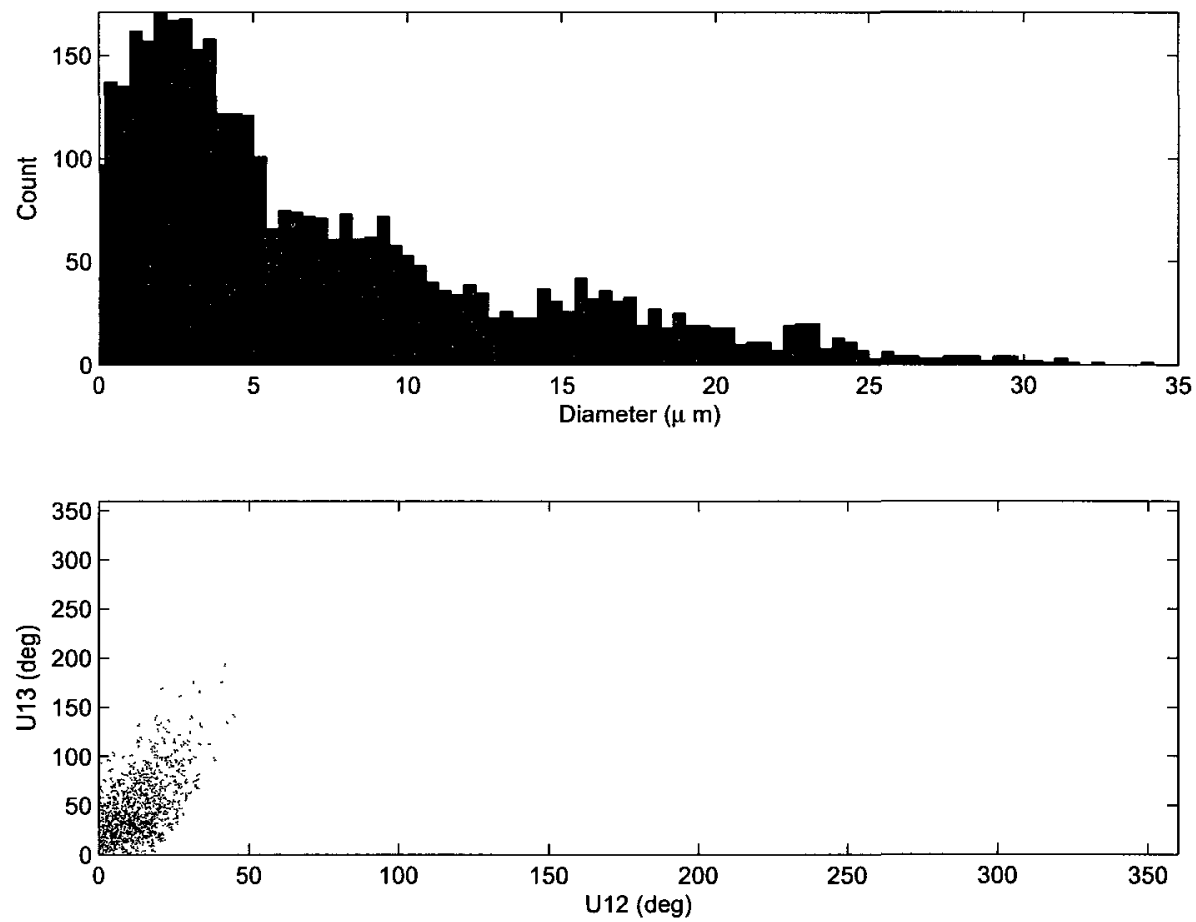

(b) Diameter histogram and PDA detector phase validation

Figure B.35: $\mathrm{x}=85 \mathrm{~mm}, \mathrm{r}=13.5 \mathrm{~mm}$ 

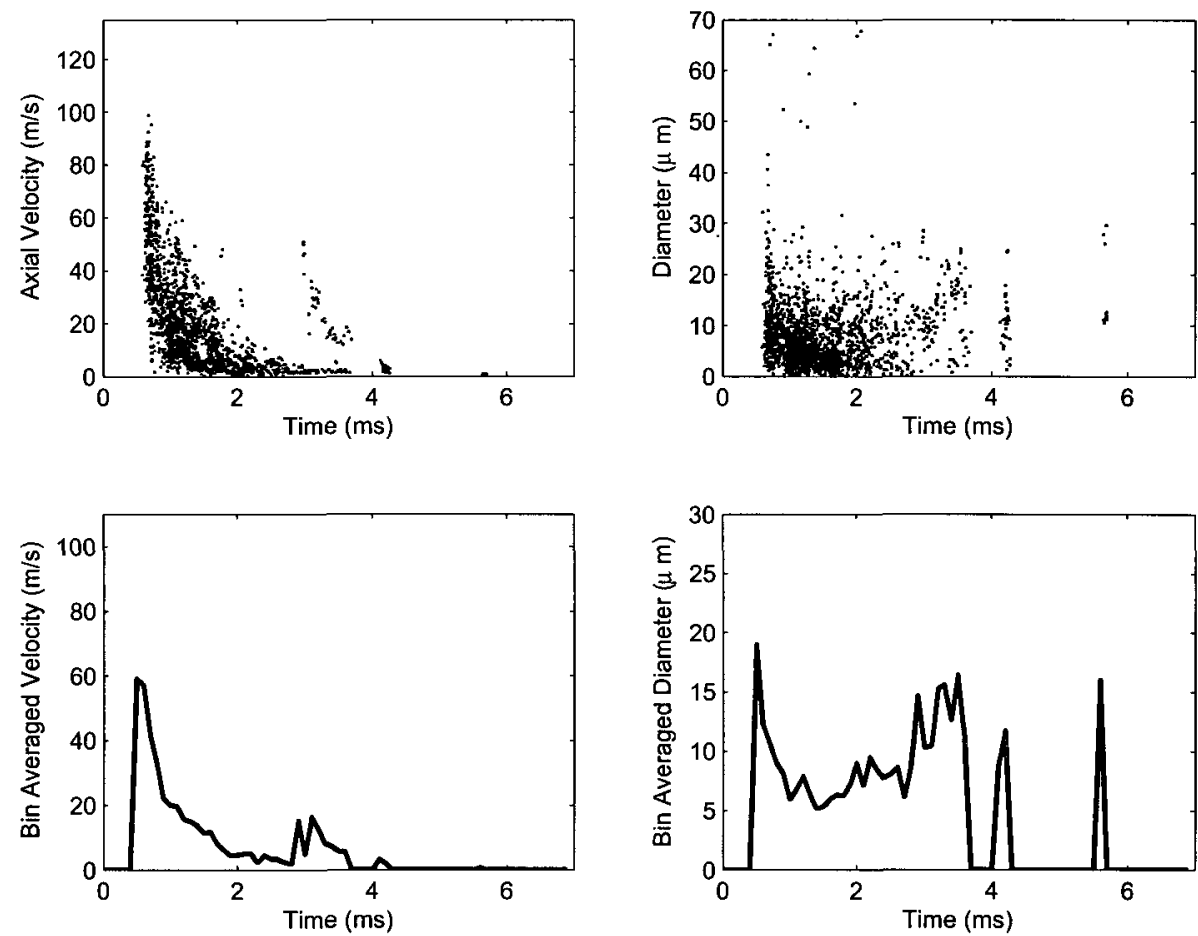

(a) Axial velocity and diameter vs time
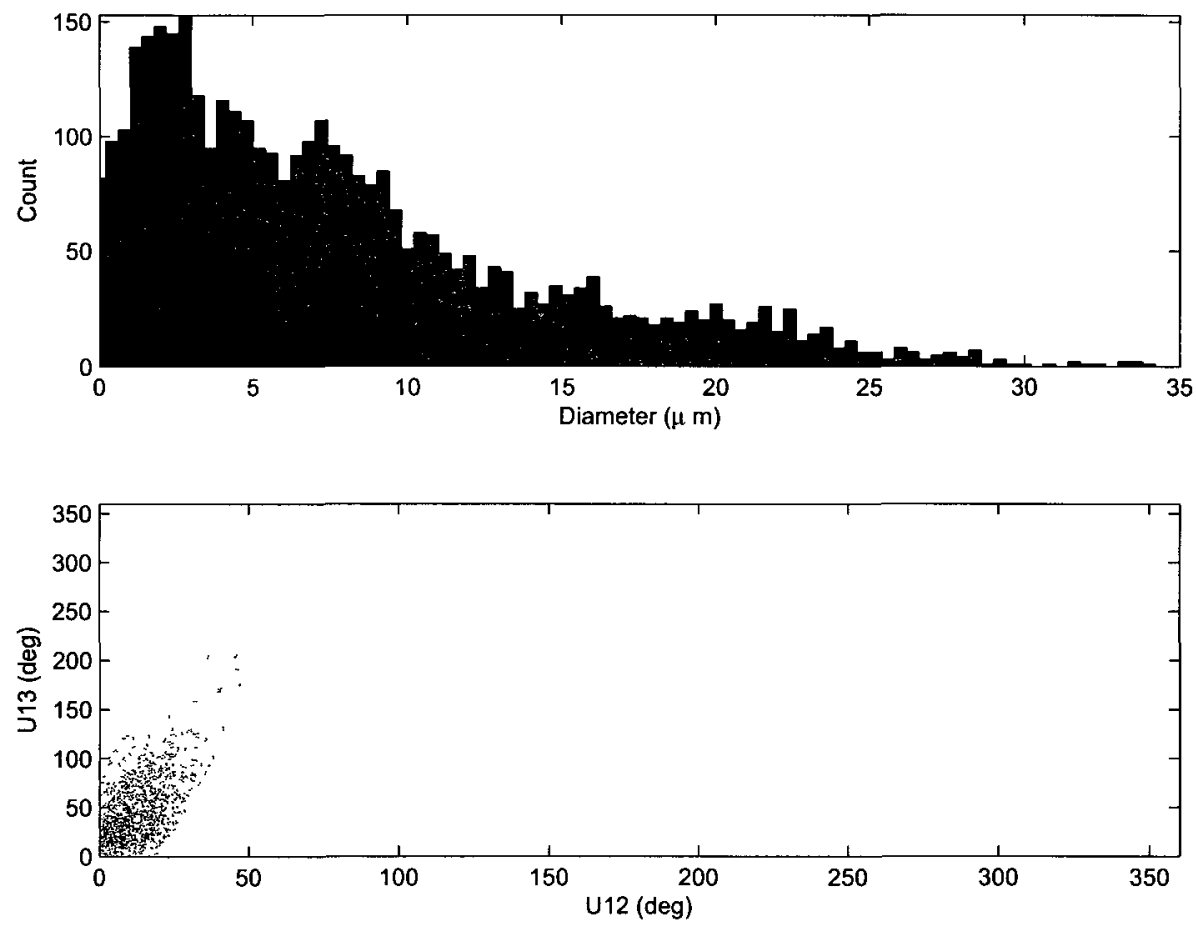

(b) Diameter histogram and PDA detector phase validation

Figure B.36: $\mathrm{x}=85 \mathrm{~mm}, \mathrm{r}=14.5 \mathrm{~mm}$ 

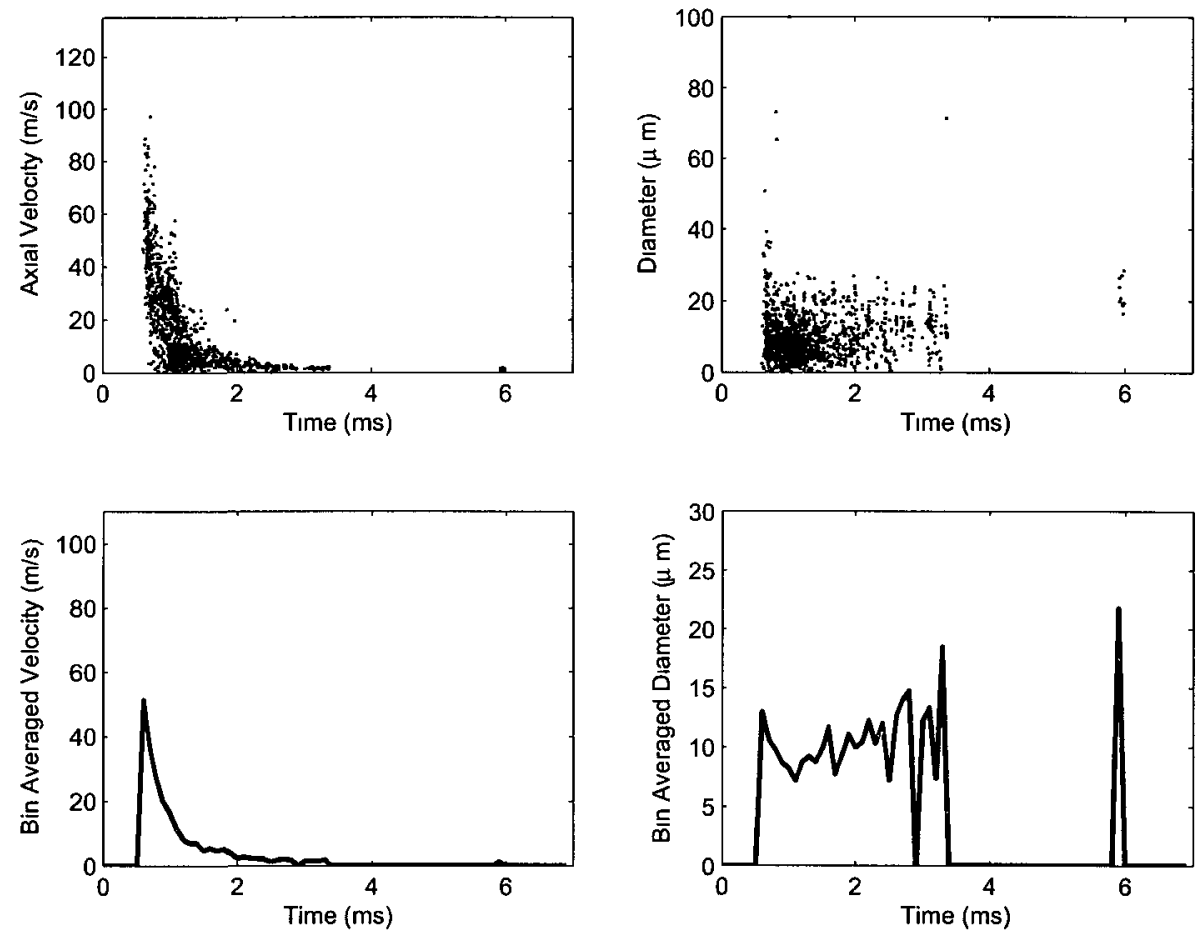

(a) Axial velocity and diameter vs time
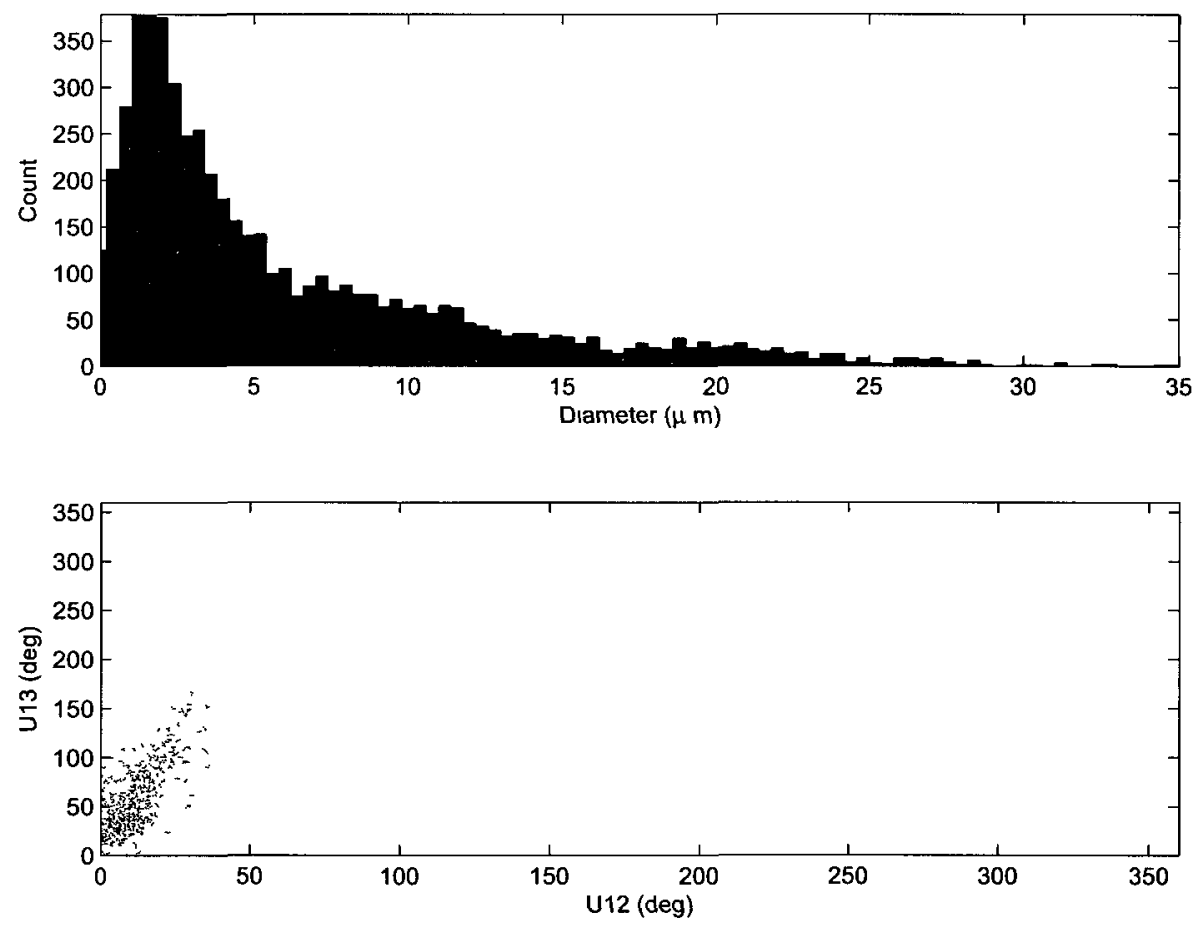

(b) Diameter histogram and PDA detector phase validation

Figure B.37: $\mathrm{x}=85 \mathrm{~mm}, \mathrm{r}=15.5 \mathrm{~mm}$ 


\section{Appendix C}

\section{Time resolved velocity profile}

01
ms
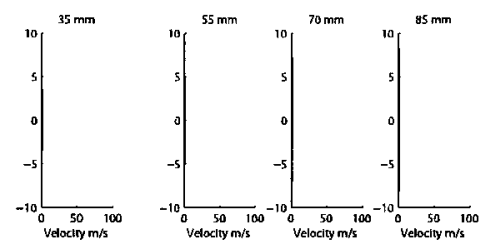

(a) Time $=0.1 \mathrm{~ms}$

$\stackrel{0.3}{\text { ms }}$
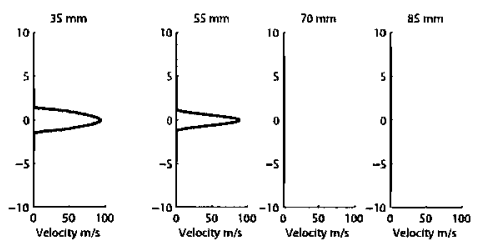

(c) Time $=0.3 \mathrm{~ms}$

0.5
$\mathrm{~ms}$
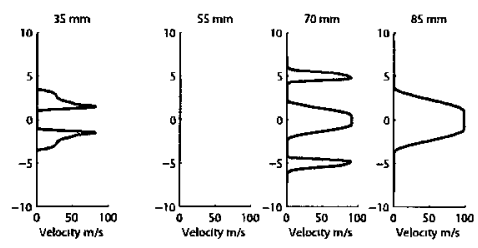

(e) Time $=0.5 \mathrm{~ms}$
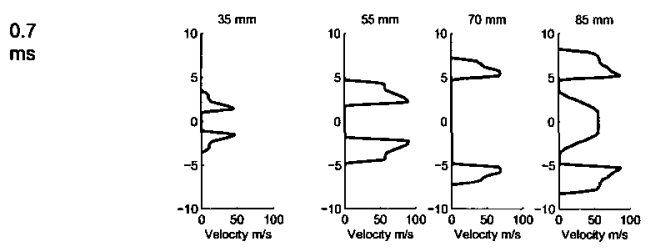

(g) Time $=0.7 \mathrm{~ms}$
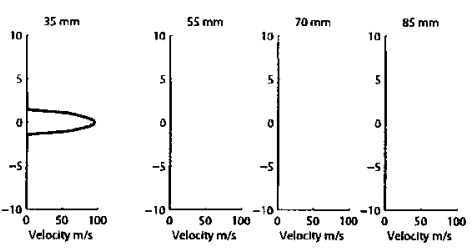

(b) Time $=0.2 \mathrm{~ms}$
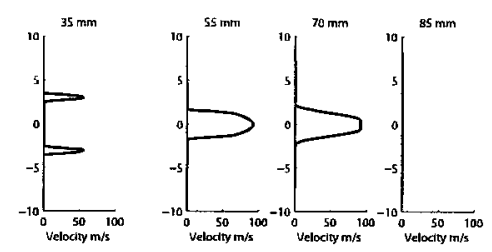

(d) Time $=0.4 \mathrm{~ms}$
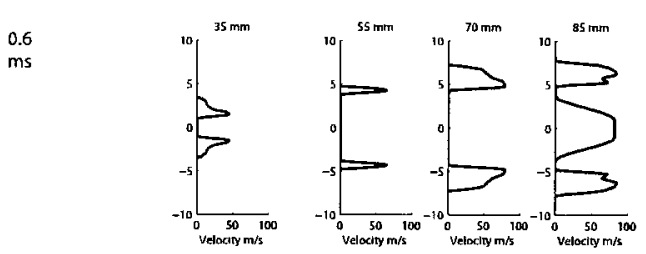

(f) Time $=0.6 \mathrm{~ms}$

0.8
$\mathrm{~ms}$
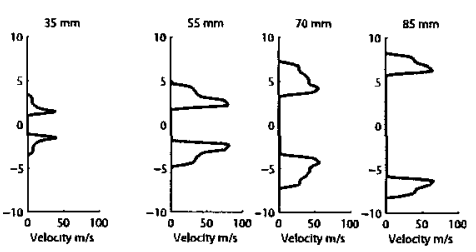

(h) Time $=0.8 \mathrm{~ms}$

Figure C.1: Time resolved velocity profile $0.1 \mathrm{~ms}$ to $0.8 \mathrm{~ms}$ 


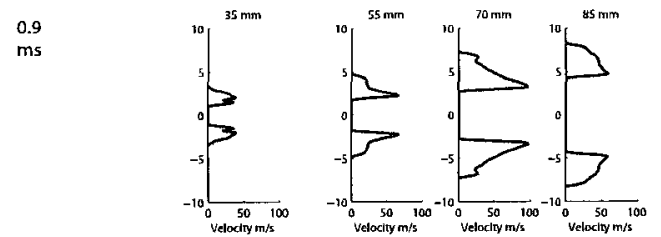

(a) Time $=0.9 \mathrm{~ms}$

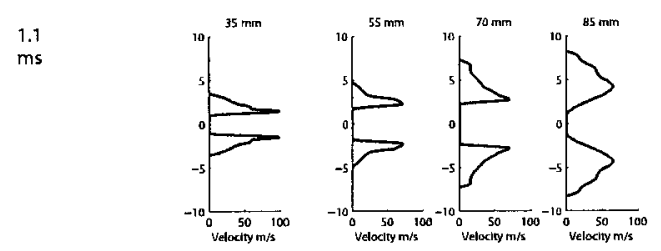

(c) Time $=1.1 \mathrm{~ms}$

1.3
$\mathrm{~ms}$

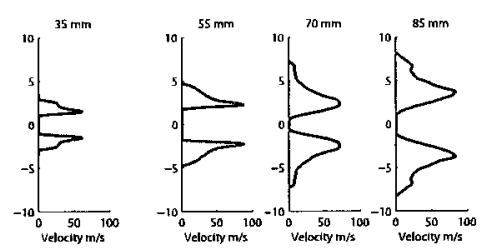

(e) Time $=1.3 \mathrm{~ms}$

1.5
$\mathrm{~ms}$

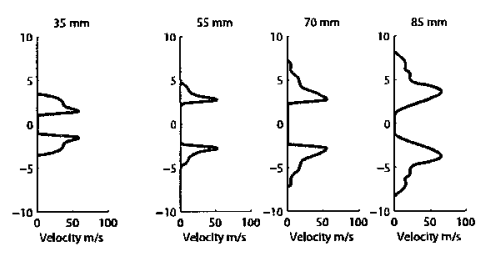

(g) Time $=1.5 \mathrm{~ms}$

1.7
ms
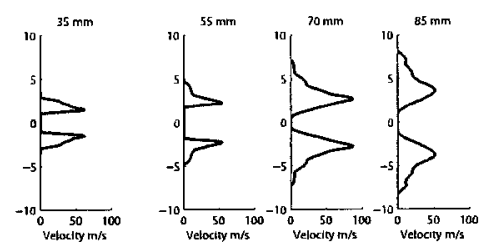

(i) Time $=1.7 \mathrm{~ms}$

1.9
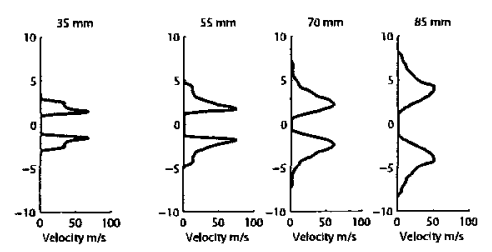

(k) Time $=1.9 \mathrm{~ms}$
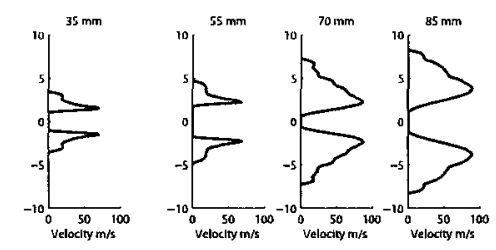

(b) Time $=1.0 \mathrm{~ms}$
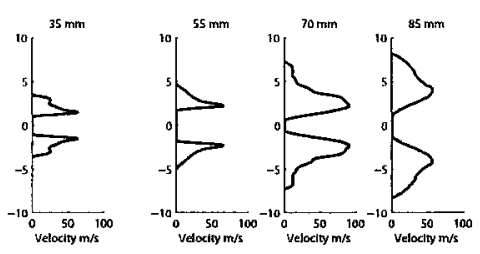

(d) Time $=1.2 \mathrm{~ms}$
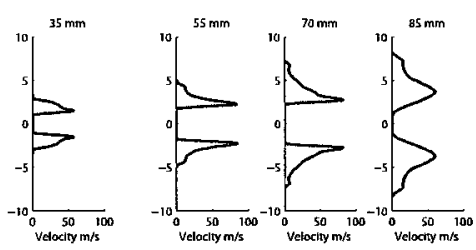

(f) Time $=1.4 \mathrm{~ms}$
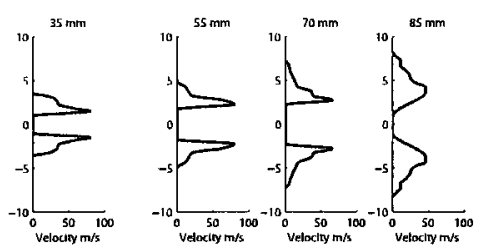

(h) Time $=1.6 \mathrm{~ms}$
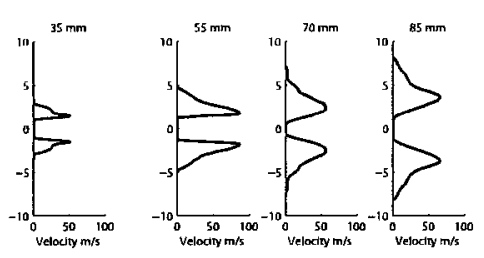

(j) Time $=1.8 \mathrm{~ms}$
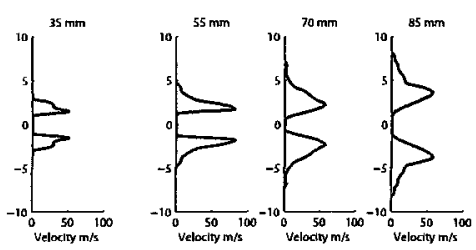

(l) Time $=2.0 \mathrm{~ms}$

Figure C.2: Time resolved velocity profile $0.9 \mathrm{~ms}$ to $2.0 \mathrm{~ms}$ 


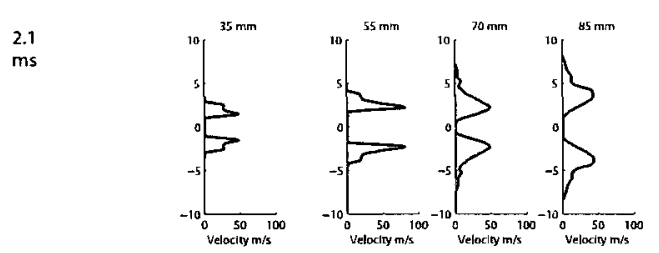

(a) Time $=2.1 \mathrm{~ms}$

$$
\begin{aligned}
& 2.3 \\
& \mathrm{~ms}
\end{aligned}
$$
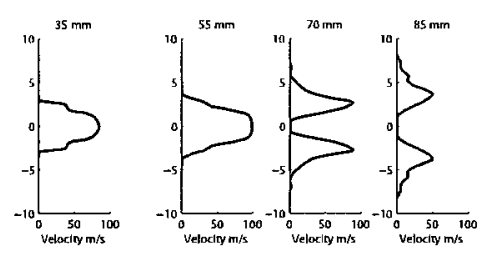

(c) Time $=2.3 \mathrm{~ms}$

2.5
$\mathrm{~ms}$
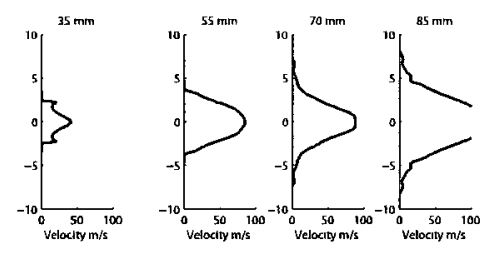

(e) Time $=2.5 \mathrm{~ms}$

2.7
$\mathrm{~ms}$
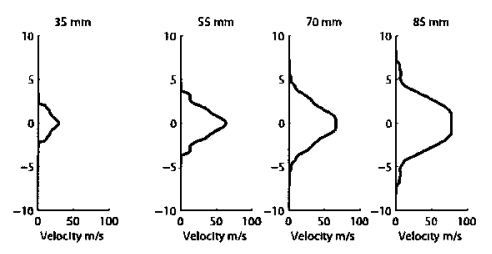

(g) Time $=2.7 \mathrm{~ms}$

2.9
$\mathrm{~ms}$
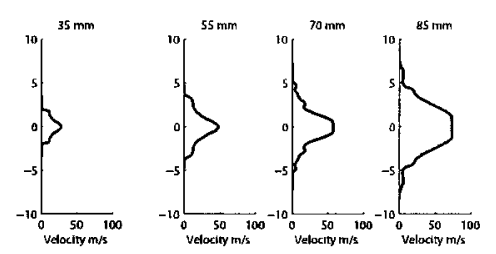

(i) Time $=2.9 \mathrm{~ms}$

3.1
$\mathrm{~ms}$

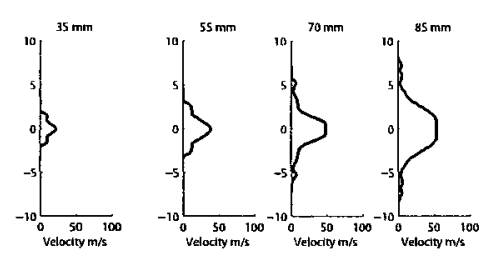

(k) Time $=3.1 \mathrm{~ms}$
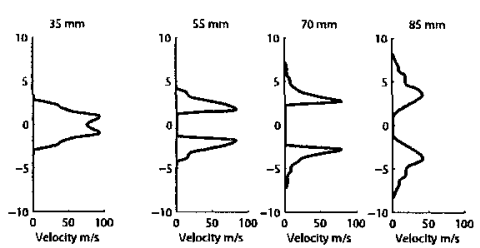

(b) Time $=2.2 \mathrm{~ms}$
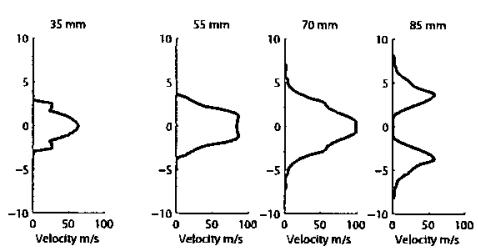

(d) Time $=2.4 \mathrm{~ms}$
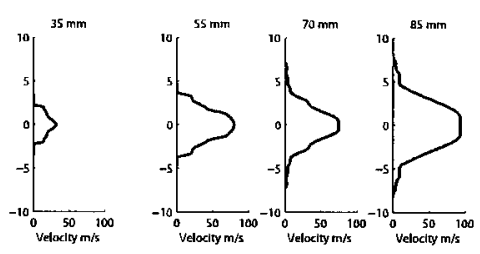

(f) Time $=2.6 \mathrm{~ms}$
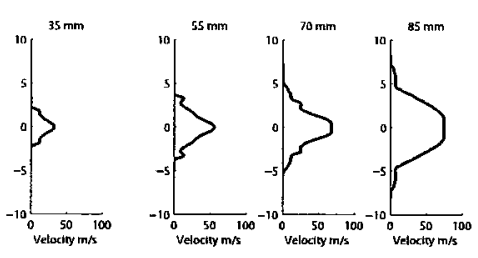

(h) Time $=2.8 \mathrm{~ms}$

3
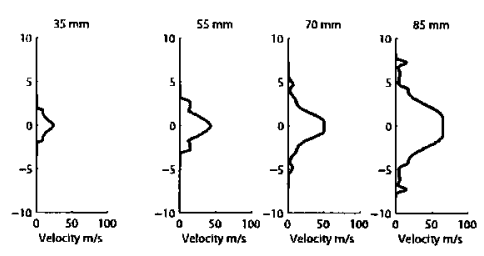

(j) Time $=3.0 \mathrm{~ms}$
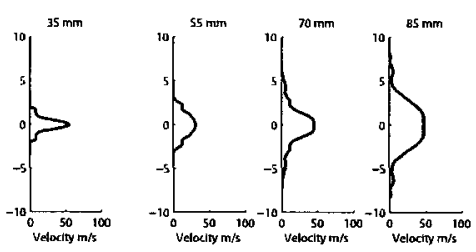

(l) Time $=3.2 \mathrm{~ms}$

Figure C.3: Time resolved velocity profile $2.1 \mathrm{~ms}$ to $3.2 \mathrm{~ms}$ 
3.3
$\mathrm{~ms}$
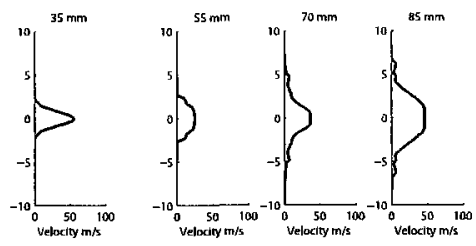

(a) Time $=3.3 \mathrm{~ms}$

3.5
$\mathrm{~ms}$
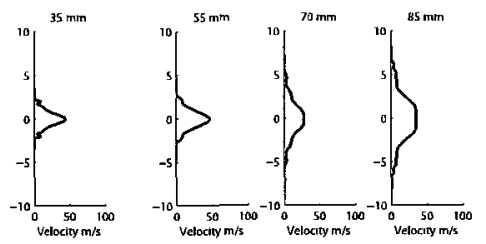

(c) Time $=3.5 \mathrm{~ms}$

3.7
$\mathrm{~ms}$
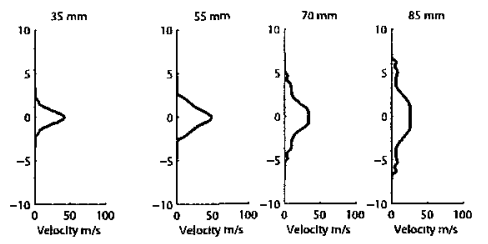

(e) Time $=3.7 \mathrm{~ms}$

3.9
$\mathrm{~ms}$
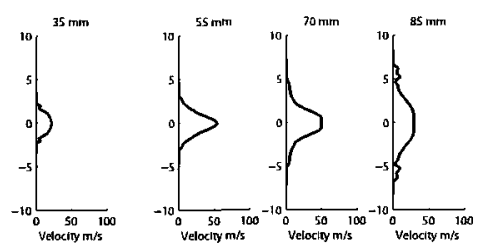

(g) Time $=3.9 \mathrm{~ms}$

4.1
ms

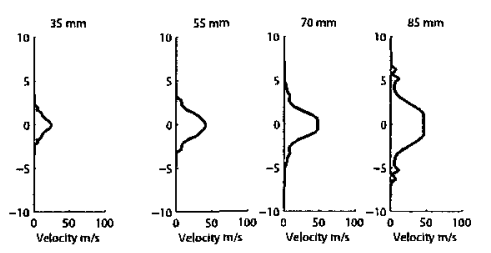

(i) Time $=4.1 \mathrm{~ms}$

4.3
ms
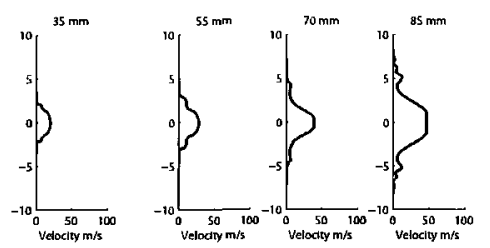

(k) Time $=4.3 \mathrm{~ms}$
3.4
$\mathrm{~ms}$
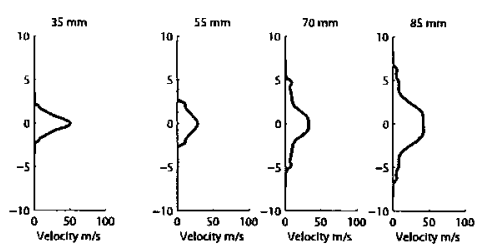

(b) Time $=3.4 \mathrm{~ms}$
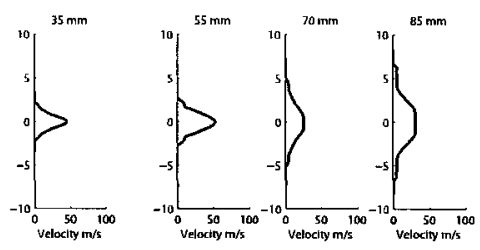

(d) Time $=3.6 \mathrm{~ms}$
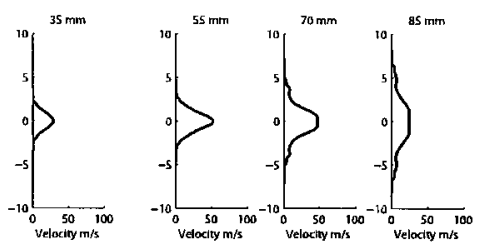

(f) Time $=3.8 \mathrm{~ms}$
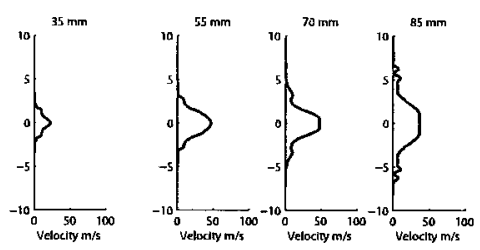

(h) Time $=4.0 \mathrm{~ms}$
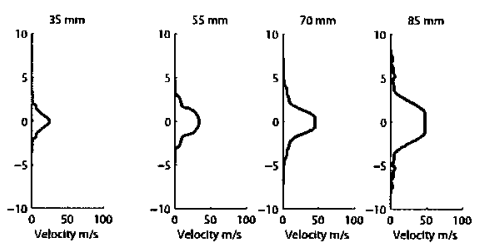

(j) Time $=4.2 \mathrm{~ms}$
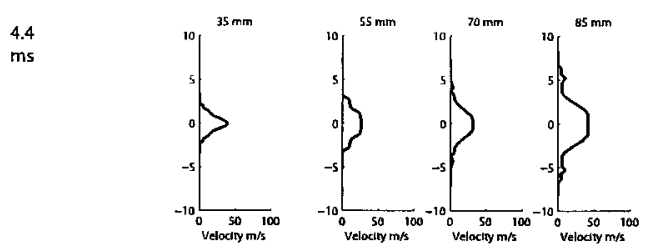

(l) Time $=4.4 \mathrm{~ms}$

Figure C.4: Time resolved velocity profile $3.3 \mathrm{~ms}$ to $4.4 \mathrm{~ms}$ 


\section{Appendix D}

\section{PDA Matlab Algorithm}

The Matlab script file reads a comma separated value file of the raw data, from a measurement position, from the BSA flow software. An example of the raw data is represented in Table. The cycle represents the injection number while the arrival time represents the time after the SOI. The transient time corresponds to the time the droplet spent inside the measurement volume. The axial velocity, radial velocity and droplet diameter are represented by $u, v$, and $d$, respectively.

The script computes spray characteristics such as; the Sauter mean diameter, arithmetic mean diameter, average velocity, and the time-resolved velocity and average mean diameter. The user must input the time bin width into the script file in order to perform the time-resolve analysis.

Table D.1: Example of raw data from the BSA flow software

$\begin{array}{cccccc}\text { Cycle } & \text { Transient time } & \text { Arrival time }(m s) & u(m / s) & v(m / s) & d(\mu m) \\ 1 & 1.8 & .003 & 130 & 22 & 34 \\ 1 & 2.1 & .006 & 35 & 4 & 12 \\ 1 & 0.9 & .007 & 114 & 11 & 6 \\ 1 & 1.1 & .090 & 65 & 6 & 45\end{array}$

